\title{
DYNAMICS OF NONLINEAR CROSS-EQUATORIAL FLOW IN THE DEEP OCEAN
}

\author{
by \\ Christopher A. Edwards \\ B.S. Haverford College \\ (1988) \\ Submitted in partial fulfillment of the \\ requirements for the degree of

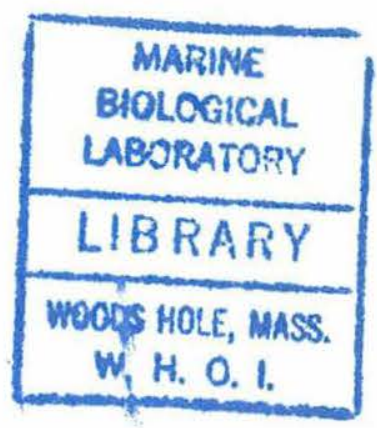 \\ Doctor of Philosophy \\ at the
MASSACHUSETTS INSTITUTE OF TECHNOLOGY
and the

WOODS HOLE OCEANOGRAPHIC INSTITUTION

December 1996
(C) 1996 Christopher A. Edwards
All rights reserved

The author hereby grants to MIT and to WHOI permission to reproduce and to distribute copies of this thesis document in whole or in part.

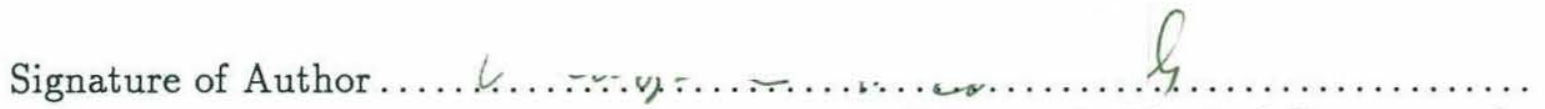

Joint Program in Physical Oceanography Massachusetts Institute of Technology Woods Hole Oceanographic Institution December 11, 1996

Certified by

John Marshall

Professor

Massachusetts Institute of Technology

Thesis Supervisor

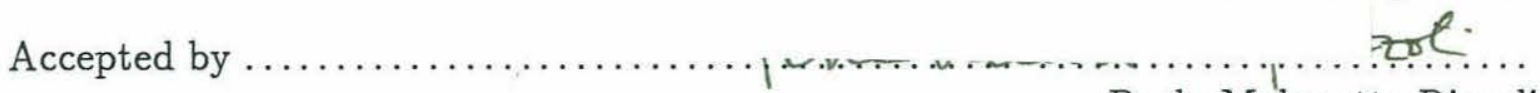
Paola Malanotte-Rizzoli Chairman, Joint Committee for Physical Oceanography Massachusetts Institute of Technology Woods Hole Oceanographic Institution 


\title{
DYNAMICS OF NONLINEAR CROSS-EQUATORIAL FLOW IN THE DEEP OCEAN
}

\author{
by \\ Christopher A. Edwards \\ Submitted in partial fulfillment of the requirements for the degree of \\ Doctor of Philosophy at the Massachusetts Institute of Technology \\ and the Woods Hole Oceanographic Institution \\ December 11, 1996
}

\begin{abstract}
The transformation of potential vorticity within and stability of nonlinear deep western boundary currents in an idealized tropical ocean are studied using a shallowwater model.

Observational evidence indicates that the potential vorticity of fluid parcels in deep western boundary currents must change sign as they cross the equator, but this evidence is otherwise unable to clarify the process. A series of numerical experiments investigate this transformation in a rectangular basin straddling the equator. A mass source located in the northwestern corner feeds fluid into the domain where it is constrained to cross the equator to reach a distributed mass sink. Dissipation is included as momentum diffusion. The Reynolds number, defined as the ratio of the mass source per unit depth to the viscosity, determines the nature of the flow, and a critical value, $R e_{c}$, divides its possible behavior into two regimes. For $R e<R e_{c}$, the flow is laminar and well described by linear theory. For Re just above the critical value, the flow is time-dependent, with cyclonic eddies forming in the western boundary current near the equator. For still larger Reynolds number, eddies of both signs emerge and form a complicated, interacting network that extends into the basin several deformation radii from the western boundary, as well as north and south of the equator.

The eddy field is established as the mechanism for potential vorticity transformation in nonlinear cross-equatorial flow. The analysis of vorticity fluxes follows from the flux-conservative form of the absolute vorticity equation. It is shown that the zonally integrated meridional flux of vorticity across the equator using no slip boundary conditions is virtually zero even in the strongly nonlinear limit suggesting that the eddies are extremely efficient vorticity transfer agents. A decomposition of the vorticity fluxes into components due to mean advection, eddy transport, and friction, reveals the growth with Reynolds number of a turbulent boundary layer that exchanges vorticity between the inertial portion of the boundary current and a frictional sub-layer where modification is straightforward.

A linear stability analysis of the shallow-water system in the tropical ocean exam-
\end{abstract}


ines the initial formation of the eddy field. The formulation assumes that the basic state is purely meridional and on a local $f$-plane. Realistic western boundary current profiles undergo a horizontal shear instability that is partially stabilized by viscosity. Calculations at several latitudes indicate that the instability is enhanced in the tropics where the internal deformation radius is a maximum. The linear stability analysis predicts a length scale of the disturbance, a location for its origin, and a critical Reynolds number that agree well with numerical results.

Thesis Supervisor: John Marshall Title: Professor, Department of Earth, Atmospheric, and Planetary Sciences, Massachusetts Institute of Technology 


\section{Acknowledgements}

In reaching this conclusion to graduate school, I am indebted to many people who substantially enriched my experience here, both professionally and personally.

First and foremost, I would like to thank Joe Pedlosky. I am grateful for his many contributions to my research and his patience in answering endless, often trivial scientific questions. It has been a great pleasure to work with him and observe his scholarship and dedication to the educational process at an institutional level, as a teacher and as a scientific mentor. Glenn Flierl has also been a frequent source of support over the last five years, kindly offering meaningful explanations of both simple and complex problems and also his good advice when asked. I thank my advisor, John Marshall, for taking me on as a student and encouraging me through times when the work progressed smoothly and when it stumbled and reached apparent impasses. Other members of my thesis committee, Mike Spall, Bruce Warren, and Nelson Hogg have contributed a considerable portion of their time and energy and made many helpful suggestions to the thesis which I appreciate.

One of the great strengths of the Joint Program is its diverse student body, and I have benefited from innumerable discussions with several members. In particular, I thank Francois Primeau and Igor Kamenkovich for their repeated explanations of various aspects of instability theory.

Financial support for this research was provided by NSF grant number OCE9115915 and ONR ASSERT grant number N00014-94-1-0844, and I received computational resources through the SCOUT Project at MIT. Alistair Adcroft generously offered his assistance in learning how to code the CM5 and significantly lessened the spin-up time for model development. Bud Brown drafted the figures in Chapter 1 adapted from other research articles.

A long list of people have made my life here at MIT and WHOI an extremely enjoyable and rewarding one. I am indebted to Paul Robbins for introducing me to 
the field of Physical Oceanography and sharing with me many of his rather eclectic interests. Natasha Beliakova, Loverboy Wardle, and Keith Alverson created a warm and stimulating work environment in which to sharpen my debating skills and discuss the latest bad hair styles. Jess kept me diving for discs. Chris provided a valuable balance and perspective for graduate school, and Raelene offered all the motivation and encouragement I needed to complete my thesis.

I am grateful also to my parents and to Julie and Jeff, for their boundless support and care as long as I can remember, and particularly since I entered graduate school. Finally I would like to thank Victor, who unassumingly provides an exceptional model of the well-rounded, young academician that I admire. 


\section{Contents}

$\begin{array}{ll}\text { Abstract } & 3\end{array}$

1 Introduction 13

1.1 Motivation . . . . . . . . . . . . . . . . . . . . . . 14

1.1.1 Hydrographic Evidence of Cross-Equatorial Flow . . . . . . 14

1.1 .2 Dynamical Motivation . . . . . . . . . . . . . 16

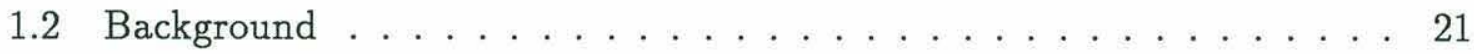

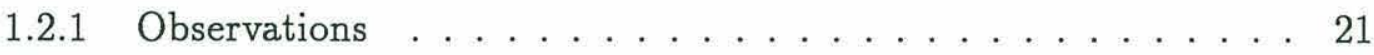

1.2 .2 Dynamical . . . . . . . . . . . . . . . 25

1.3 Outline of the Thesis . . . . . . . . . . . . . . 27

2 The Model $\quad 29$

2.1 Scaling Ertel's Potential Vorticity . . . . . . . . . . . . . . . . 29

2.2 The Shallow-Water Model . . . . . . . . . . . . . . . . . 31

2.3 Model Configuration and Scaling . . . . . . . . . . . . . 34

2.4 The Vorticity Equation and Reynolds Number . . . . . . . . . . . 36

2.5 The Numerical Model . . . . . . . . . . . . . . . . . . . . . . . 38

2.5 .1 Spatial Discretization . . . . . . . . . . . . . 38

2.5.2 Numerical Boundary Condition . . . . . . . . . . . . 41

2.5.3 Parallel Programming . . . . . . . . . . . . . . 42 
3 Linear to Nonlinear Cross-equatorial Flow 45

3.1 Introduction . . . . . . . . . . . . . . . . . . 45

3.2 Spin-up of the Equatorial Ocean . . . . . . . . . . . . . . 46

3.3 Linear Solution and Numerical Experiment . . . . . . . . . . . . . 51

3.4 Transition to Instability $\ldots \ldots \ldots 6$

3.5 Strongly Nonlinear Flow . . . . . . . . . . . . . . . . . . . . . . 62

3.6 Summary . . . . . . . . . . . . . . . . . . . 72

4 Vorticity Analysis and Sensitivity 73

4.1 Time-averaged Vorticity Analysis . . . . . . . . . . . . . . 73

$4.1 .1 \quad$ J-vectors . . . . . . . . . . . . . . . . . . . 73

4.1.2 Analysis and Numerical Results . . . . . . . . . . . . 75

4.1.3 Balances from the Time-Mean Vorticity Equation . . . . . . . . 92

4.2 Localized Upwelling . . . . . . . . . . . . . . . . . . . . . . . . . . . 97

4.3 Free-slip Boundary Conditions . . . . . . . . . . . . . . . 103

4.4 Barotropic Limit . . . . . . . . . . . . . . . . . . . . . . . 110

4.5 Summary . . . . . . . . . . . . . . . . . . 113

5 The Tropically Enhanced Instability of the Western Boundary Current

5.1 Introduction . . . . . . . . . . . . . . . . . . . . 115

5.2 Formulation of the Problem . . . . . . . . . . . . . . . . 120

5.3 Inviscid Shear Instability . . . . . . . . . . . . . . . . . . . . 124

5.3 .1 Numerics . . . . . . . . . . . . . . . . . . . . . 125

5.3.2 Instability of the Munk Boundary Layer . . . . . . . . 126

5.3.3 Geostrophic Perturbations . . . . . . . . . . . . . . . . . 133

5.3.4 Instability of an Inertio-Viscous Current . . . . . . . . . . 139

5.4 The Influence of Viscosity . . . . . . . . . . . . . . . . . 142

5.5 Inertial Instability . . . . . . . . . . . . . . . . . . 151 
5.6 Summary and Discussion . . . . . . . . . . . . . . . . 154

6 Conclusion $\quad 159$

6.1 Summary and Discussion of Thesis . . . . . . . . . . . . 159

6.2 Extension to Full Three-Dimensional Fluid . . . . . . . . . . . . . . . 164

$\begin{array}{ll}\text { A Numerical Approximations } & 167\end{array}$

A.1 Model Time-Stepping . . . . . . . . . . . . . . 167

A.2 Grid Stretching . . . . . . . . . . . . . . . . 169

A.3 Numerical Approximation to Vorticity Components . . . . . . . . . . 173

A.4 Numerical Approximation to J-vectors . . . . . . . . . . . . . . 174

$\begin{array}{ll}\text { References } & 176\end{array}$ 


\section{List of Figures}

1.1 GEOSECS Atlantic Expedition, Western Atlantic salinity section . . . 15

1.2 Stommel and Arons global abyssal circulation . . . . . . . . . 18

1.3 SOFAR float composite velocity profile . . . . . . . . . . . . 19

1.4 Equatorial tracer tongue . . . . . . . . . . . . . . 23

1.5 SOFAR float displacement vectors . . . . . . . . . . . . 24

2.1 Model Configuration . . . . . . . . . . . . . . . . . 35

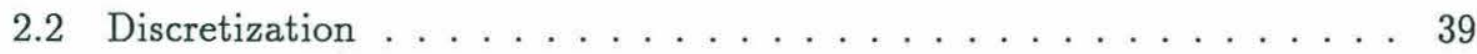

3.1 Linear Spinup . . . . . . . . . . . . . . . . . . 48

3.2 Height difference, linear . . . . . . . . . . . . . . . . . . 50

3.3 Nonlinear Spinup . . . . . . . . . . . . . . . . . . . 52

3.4 Mean height difference, nonlinear . . . . . . . . . . . . . . 53

3.5 Subdomain A . . . . . . . . . . . . . . . . 56

3.6 Stommel and Arons, analytical . . . . . . . . . . . . . 58

3.7 Run U1, linear steady state . . . . . . . . . . . . . . . 59

3.8 Model Run U3, uh . . . . . . . . . . . . . . . . . 61

3.9 Model Run U3, uhbar . . . . . . . . . . . . . . . 63

3.10 Model Run U8, instantaneous . . . . . . . . . . . . . . . 65

3.11 Model Run U8, instantaneous PV . . . . . . . . . . . . . 67

3.12 Model Run U8, time-averaged . . . . . . . . . . . . . . 68

3.13 Model Run U3, time-averaged meridional transport . . . . . . . . . . . 69 
3.14 Model Run U8, time-averaged meridional transport . . . . . . . . . . 69

3.15 Mean Potential Vorticity, $\operatorname{Re}=500 \ldots \ldots$. . . . . . . . . 71

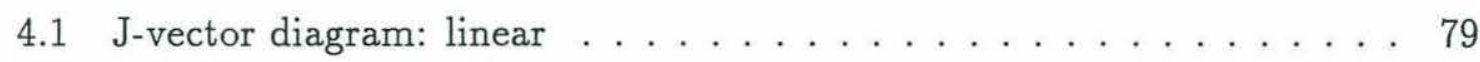

4.2 J-vectors: $\mathrm{U} 1 \ldots \ldots \ldots$

4.3 Streamfunction of $\mathrm{J}: \mathrm{U} 1 \ldots \ldots \ldots$

4.4 Streamfunction of J: $\mathrm{U} 2 \ldots \ldots \ldots$

4.5 Streamfunction of J: U8 $\ldots \ldots \ldots \ldots$

4.6 J-vector components: U8 . . . . . . . . . . . . . . . . . . . . . . 84

4.7 J-vector component divergences: U8 . . . . . . . . . . . . . 86

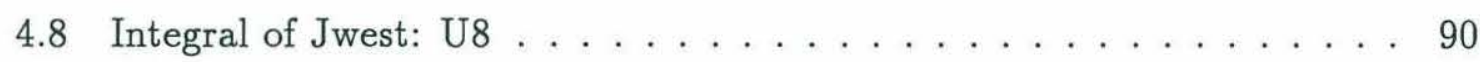

4.9 Integral of Jnorth: U8 $\ldots \ldots \ldots \ldots \ldots$

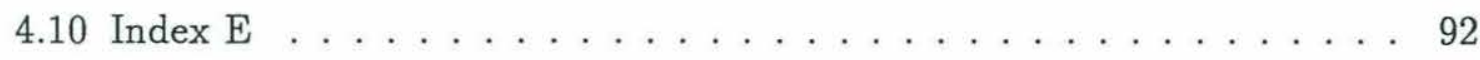

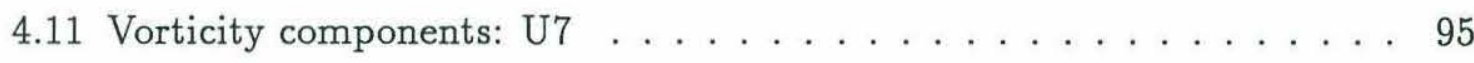

4.12 Vorticity balance: $\mathrm{U} 7 \ldots \ldots \ldots$

4.13 Localized upwelling distribution . . . . . . . . . . . . . . 98

4.14 Velocity and height fields: N2 . . . . . . . . . . . . . . . 99

4.15 Potential vorticity: N2 . . . . . . . . . . . . . . . 100

4.16 Time-mean velocity and height fields: N2 . . . . . . . . . . . . 101

4.17 Vorticity flux streamfunction: N2 . . . . . . . . . . . . . . 102

4.18 Mean velocity and height contours: UFS . . . . . . . . . . . . 104

4.19 Vorticity flux streamfunction: UFS . . . . . . . . . . . . . 105

4.20 Integrated vorticity flux: UFS . . . . . . . . . . . . . 106

4.21 Vorticity balance: UFS . . . . . . . . . . . . . . . . . 108

4.22 Eddy fluxes: UFS . . . . . . . . . . . . . . . . . . . . . 109

4.23 Model UBar: instantaneous velocity and height . . . . . . . . . . 112

5.1 Looper floats . . . . . . . . . . . . . . . . . . . . 116

5.23 instantaneous velocity and height fields $\ldots \ldots \ldots \ldots \ldots$ 
5.3 Munk B.L.: mean velocity and potential vorticity gradient . . . . . 127

5.4 Munk B.L.: Growth rates and phase speeds . . . . . . . . . . 128

5.5 Munk B.L.: Eigenfunctions . . . . . . . . . . . . . . . . . 130

5.6 Munk B.L.: Growth rate and phase speed vs latitude . . . . . . . 132

5.7 Growth rate and phase speed for geostrophic perturbations . . . . . . 135

5.8 Generic growth rate . . . . . . . . . . . . . . . . . 136

5.9 Western boundary with effective beta . . . . . . . . . . 138

5.10 Model output: Growth rate and phase speed vs latitude . . . . . . . . 141

5.11 Model output: Viscous growth rate and phase speed vs wavenumber . . 143

5.12 Model output: Viscous eigenfunctions . . . . . . . . . . . . . 145

5.13 Model output post instability: delta height . . . . . . . . . . . . . 146

5.14 Velocity and height field: narrow boundary layer . . . . . . . . . . 147

5.15 Growth rates and phase speeds: narrow boundary layer . . . . . . . . 148

5.16 Evolution of eddy development . . . . . . . . . . . . 150

5.17 Munk B.L., geostrophic perturbations with beta . . . . . . . . . 156

A.1 X Grid information and derivatives . . . . . . . . . . . . 172

A.2 Y Grid information and derivatives . . . . . . . . . . . . . 172 


\section{Chapter 1}

\section{Introduction}

During intense winter storms over the Labrador and Greenland Seas, water cools and convectively adjusts, sinking to great depth. In so doing, it begins a complicated journey through the world's oceans, where it eventually rises and returns to these high latitudes only to begin the cycle again. Along its path, most of this water necessarily crosses the equator, at least twice in the Atlantic, and perhaps also in the Pacific and Indian oceans. Although the fact of deep equatorial crossing is well accepted, the details of the process remain poorly understood from both observational and dynamical perspectives. The breakdown of the geostrophic approximation at low latitudes leads to a large uncertainty in the velocities obtained by hydrographic surveys in the vicinity, and the strong time-dependence of observed currents makes mean field calculations from current measurements particularly questionable. Analytical studies have successfully modeled near-equatorial currents under very restrictive conditions, including linear and purely inviscid dynamics. However, the dynamical regime that most likely applies to the ocean is the intermediate one, in which inertial motion is significant and dissipation weak but necessarily non-zero.

The purpose of this thesis is to analyze cross-equatorial flow under these more general dynamical conditions. With the basic assumption that fluid must cross the equator, the question of interest is how. The problem is motivated specifically in the 
context of the thermohaline circulation. The surface and bottom circulations near the equator are fundamentally different in that the local forcing by wind and topography contributes to the vorticity balance and thereby changes the dynamical constraints of the flow. This work concentrates on non-locally driven currents, with the deep flow of the Atlantic in mind. It is a process-oriented study, numerical in nature and highly idealized in context, intended to explain dynamically how fluid crosses the equator. In particular, it determines how fluid parcels, carried in intense cross-equatorial currents and constrained by only weak dissipation, modify their potential vorticity along their path. The thesis will show that this process takes place through the development of a turbulent boundary layer that resides in the inertial portion of the western boundary current and that transfers vorticity from this outer region to an inner, frictional layer, where it is removed from the basin through lateral dissipation.

\subsection{Motivation}

\subsubsection{Hydrographic Evidence of Cross-Equatorial Flow}

A considerable body of evidence supports the notion that fluid travels from hemisphere to hemisphere in the ocean. A particularly compelling argument is found in any meridional hydrographic section of the Atlantic Ocean. Shown in Figure 1.1 is one such section (salinity) from the Atlantic GEOSECS Atlas (Bainbridge (1980)). Using salinity as a proxy for water mass reveals that several different water masses are found in the Atlantic both north and south of the equator. In particular, the high salinity tongue of North Atlantic Deep Water (NADW) plunges to depth from its high latitude formation region near the surface southward and is visible between depths of 1500 and 3500 meters at the equator. Sandwiching this NADW are two layers of low salinity fluid of southern hemisphere origin: Antarctic Bottom Water and Antarctic Intermediate Water.

That water of similar origin is found on either side of the equator invites the 

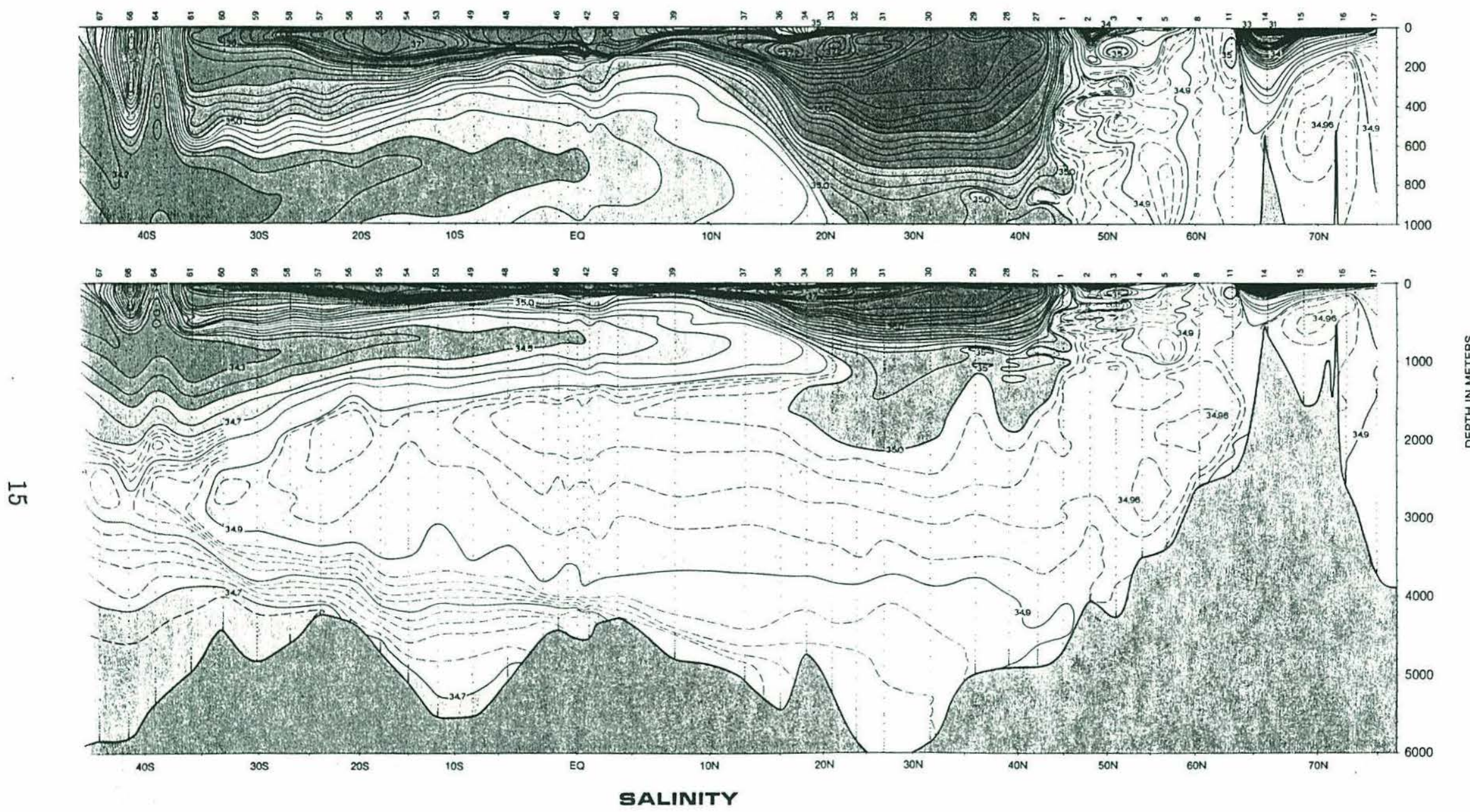

Figure 1.1: Section of salinity from the western Atlantic Ocean showing interhemispheric penetration of interleaving salinity signatures. Reproduced from the Atlantic GEOSECS Atlas (Bainbridge (1980)). 
question of its route, which can not be inferred from this section alone, but which can be anticipated from dynamical considerations or illustrated through additional observations.

\subsubsection{Dynamical Motivation}

\section{Viscous limit}

Certainly the most well-known and arguably the most widely-accepted theory of deep flow in the ocean is that of Stommel and Arons (1960a). In this framework as in the closely related homogenous ocean circulation theories, the circulation is divided between interior and boundary layer features. The interior flow is governed by a linear vorticity balance,

$$
\beta v=f \frac{w_{\star}}{H},
$$

where $f$ is the Coriolis parameter, $\beta$ its meridional derivative, $v$ is the meridional velocity, $H$ the fluid-layer depth, and $w_{\star}$ the vertical velocity at the top of the layer. This upwelling is the specified forcing of the system, and it represents a distributed vertical velocity whose basin-wide integral balances the volume transport of fluid sinking in high-latitude, convective regions. The theory assumes a level bottom so the vertical velocity there is zero. Appended to this interior flow is a boundary current that redistributes mass within the basin and which can be shown to be dynamically consistent only at the western boundary.

The physics of this boundary current was left unspecified in the original theory, but its existence and associated transport are robust features of the model. Assuming that the boundary layer dynamics are distinct from the interior flow, the boundary layer transport is determined entirely by the interior flow and spatial distribution of sources and sinks that supply and remove fluid in the deep ocean. The boundary current can be in principle either inertial or frictional. It will be shown below that a successful model of cross-equatorial flow must include some friction. At one extreme is 
the purely linear example of the Munk (1950) parameterization. Modelling the oceanic dissipation as a large-scale diffusive process, the boundary layer velocity is determined by

$$
\beta v=A_{H} \frac{d^{3} v}{d x^{3}} .
$$

Here meridional motion (i.e., planetary vorticity advection) is balanced by vorticity diffusion, and the boundary layer has a zonal scale,

$$
\delta_{M}=\left(\frac{A_{H}}{\beta}\right)^{\frac{1}{3}} .
$$

The dynamics of this model are asymptotically consistent with the full equations of motion provided that the inertial boundary layer scale,

$$
\delta_{I}=\sqrt{\frac{U}{\beta}},
$$

where $U$ is the scale of the zonal velocity, is small compared to the frictional scale, $\delta_{M}$. In the linear theory, currents can be made arbitrarily weak to satisfy this constraint. At the equator in this model, a weak zonal flow in the interior remains, and the boundary layer transport reflects the geographical location of the sources and sinks, taking fluid from hemisphere to hemisphere as required by the global balance. There is no difficulty modifying vorticity in this frictional model. One conceptualization of the global deep circulation of the ocean is shown in Figure 1.2 reflecting these dynamics. Notice that the fluid travels unhindered across the equator in each ocean.

Although this theory is successful in predicting a global circulation, it requires very weak currents to validate the linear dynamics used. From the full vorticity equation for a homogeneous ocean,

$$
\zeta_{t}+\vec{u} \cdot \nabla \zeta+\beta v+(f+\zeta) \nabla \cdot \vec{u}=A_{H} \nabla^{2} \zeta
$$

where $\zeta$ is the relative vorticity of the flow, it is clear that a model that neglects relative vorticity requires a small value for the ratio,

$$
\begin{aligned}
R & =\frac{U}{\beta \delta_{x}^{2}} \\
& =\frac{V}{\beta \delta_{y} \delta_{x}} .
\end{aligned}
$$




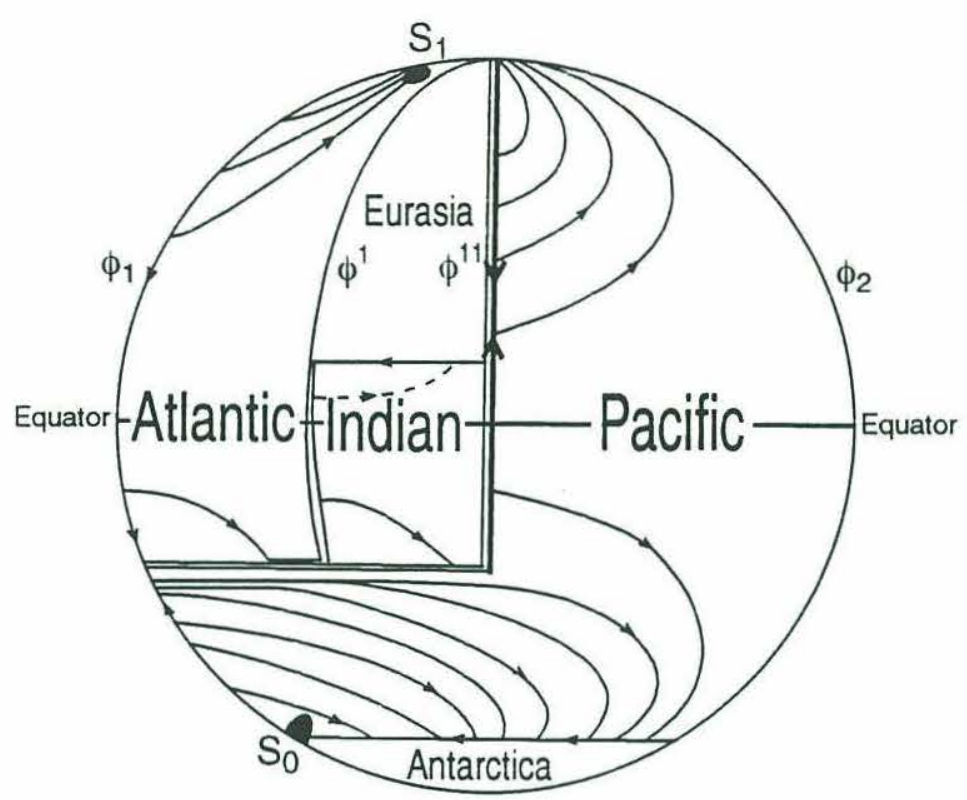

Figure 1.2: Schematic representation of the global abyssal circulation predicted by Stommel and Arons. Note the cross-equatorial flow indicated in each ocean. Adapted from Stommel and Arons (1960b).

Here, $\delta_{x}$ and $\delta_{y}$ represent the zonal and meridional length scales over which the fields vary. It is possible to estimate from observations these scales to determine if the dynamical assumptions of the theory are consistent.

Figure 1.3 presents a composite velocity profile for the along-boundary component in the tropical Atlantic ocean at 1800 meters depth taken from SOFAR floats (Richardson and Schmitz (1993)). There exists a strong southward peak roughly $30 \mathrm{~km}$ from the continental slope, with a maximum speed of $26 \mathrm{~cm} / \mathrm{s}$. In their text the authors note that the full data were averaged into $10-\mathrm{km}$-wide bins, and that the peak velocities reached $55 \mathrm{~cm} / \mathrm{s}$. The variation of this velocity scale occurs on a $50 \mathrm{~km}$ length scale. The velocity perpendicular to the boundary, $U$, and the meridional length scale, $\delta_{y}$, are not well known from observations, but using $V=.5 \mathrm{~m} / \mathrm{s}, \delta_{x}=50 \mathrm{~km}, \beta=2 \times 10^{-11}$ $\mathrm{m}^{-1} \mathrm{~s}^{-1}$, and $\delta_{y}=1000 \mathrm{~km}$, gives

$$
R=\frac{1}{2}
$$

This $O(1)$ value suggests that the assumption of linear dynamics in the boundary 

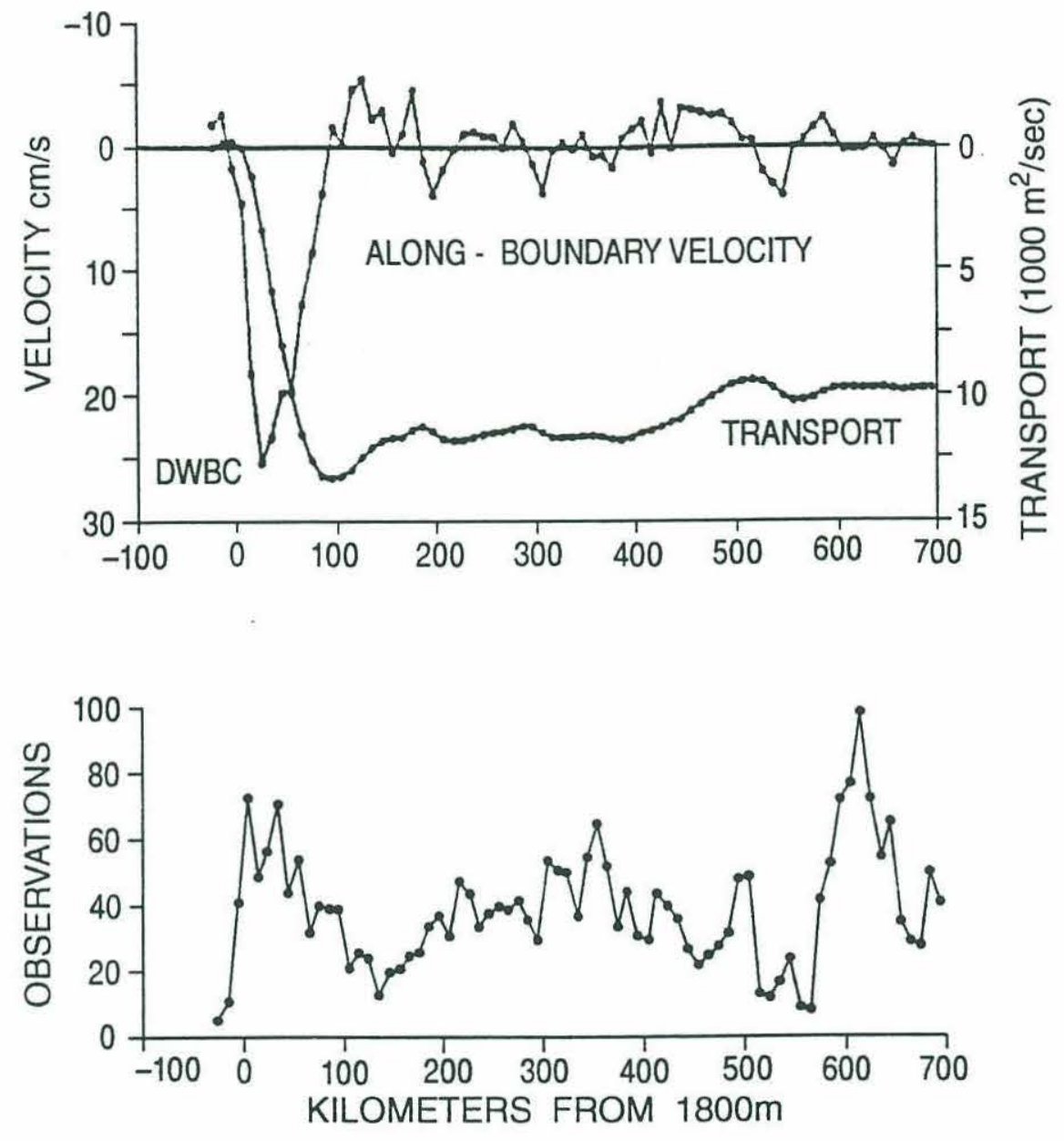

Figure 1.3: The average along-boundary velocity, transport per unit depth, and number of observations from the $1800 \mathrm{~m}$ isobath compiled from nine near-equatorial SOFAR floats at 1800 meters depth in the western Atlantic Notice the intense western boundary current peaked near $x=40 \mathrm{~km}$ with the substantial decline in velocity immediately adjacent to the coast. The maximum velocity in the DWBC was $55 \mathrm{~cm} / \mathrm{s}$, though the maximum in the $10 \mathrm{~km}$-wide averaged bins is 26 cm/s. Figure adapted from Richardson and Schmitz (1993). 
current is inconsistent with available observations. Regardless of the magnitude of the friction, nonlinearity must be considered for the boundary current structure and associated dynamics of cross-equatorial flow.

\section{Inviscid limit}

Without any fundamental understanding of the magnitude of dissipation in the ocean, it is tempting to take an opposing view and consider an ocean in which dissipation is entirely neglected. Under this assumption, the central dynamical issue of crossequatorial flow becomes the transformation of potential vorticity. This quantity is defined by

$$
P V=-\frac{1}{\rho_{0}}(2 \Omega+\omega) \cdot \nabla \rho,
$$

where $\rho$ is the fluid density, $\rho_{0}$ a reference value, $\Omega$ is the earth's rotation vector, and $\omega$ is the full three-dimensional curl of the velocity field. The potential vorticity is exactly conserved by fluid parcels in the absence of forcing or dissipative processes. As a result, inviscid and unforced meridional motion on the sphere must coincide with changes in the relative vorticity of the flow or in the stratification of the fluid, or both. At mid-latitudes the change in the Coriolis parameter $(f=2 \Omega \sin \phi$, where $\phi$ is the latitude) is relatively small compared to its magnitude, and small changes in the stratification can accomodate large meridional excursions.

However, across the equator the projection of $\Omega$ on the local vertical changes sign, and the fluid must compensate by creating substantial relative vorticity or by inverting the density field. Far from the western boundary current and far from the equator such as in the large portions of the section in Figure 1.1, relative vorticity is weak compared to planetary vorticity, and the ocean is stably stratified. Assuming that fluid parcels of similar origin once had similar potential vorticity, it is clear that potential vorticity modification is fundamental to cross-equatorial flow. Somewhere

along their route from hemisphere to hemisphere, fluid parcels must change the sign of their potential vorticity. As this change can only be achieved through some form 
of forcing or dissipation, the process of cross-equatorial flow is found incompatible with the assumption of an inviscid ocean. Ruling out local forcing since this study is motivated by deep ocean dynamics, dissipation must be included in any successful discussion of cross-equatorial flow.

As shown in the previous section, the boundary layer dynamics are in nature at least partially inertial. However, some dissipation is required to change the sign of the potential vorticity. Thus the ocean operates in an intermediate regime, both inertial and frictional.

\subsection{Background}

\subsubsection{Observations}

Owing to the impact of tropical ocean dynamics on global weather patterns, there exists a sizeable number of observations of the tropical ocean. However, these studies are primarily devoted to the surface circulation and atmospheric fluxes, not the deep ocean in the critical'western boundary region. Some observational studies have been directed toward this region and are found in hydrographic sections and float measurements. Relying on thermal wind balance to deduce currents from density gradients, indirect estimates from hydrographic surveys are limited near the equator by the failure of the geostrophic approximation there.

Some hydrographic measurements are not dependent on this balance and therefore are particularly intriguing. For example, the measurement of $\mathrm{CCl}_{3} \mathrm{~F}$ on the $\sigma_{1.5}=34.63 \mathrm{~kg} \mathrm{~m}^{-3}$ surface in the the core of Upper North Atlantic Deep Water (approximately 1600 meters) by Weiss et al. (1985) presents an indirect measurement of the deep equatorial transport. Reproduced in Figure 1.4 along with subsequent data collected five years later (Weiss et al. (1989), Weiss et al. (1991), and Weiss et al. (1993)), contours of high chloroflouromethane snake down the western boundary of the North and South Atlantic and are drawn out in an elongated feature directly on 
the equator. The time-evolution of this equatorial tongue indicated by a comparison of the figures is unmistakable. Clearly some fluid in the deep western boundary current is directed eastward upon reaching the equator, while the remaining fraction travels directly southward across the equator.

This tracer tongue may reflect an Eulerian current in which the actual circulation of the tropical ocean involves an along equatorial branch, or instead it may indicate a Lagrangian pathway from the boundary to the interior ocean in which equatorial waves draw fluid away from the western boundary preferentially at the equator. The original work calculated that if the feature reflected the former possibility, it indicated a mean flow for the boundary current of approximately $1.4 \mathrm{~cm} \mathrm{~s}^{-1}$ (Weiss et al. (1985)).

McCartney (1993) compiled data from 3 hydrographic surveys that bracket the equatorial Atlantic in an effort to estimate the mean circulation of the deep tropics. Results indicated an intense deep western boundary current, transporting $35 \times$ $10^{6} \mathrm{~m}^{3} \mathrm{~s}^{-1}(35 \mathrm{~Sv})$ of NADW, with a northward-flowing counter-current immediately east of the DWBC, returning up to $20 \mathrm{~Sv}$. From property distributions and geostrophic shear calculations at $6^{\circ} \mathrm{N}, 11^{\circ} \mathrm{S}$, and between $2^{\circ}$ and $5^{\circ} \mathrm{S}$ of the equator, the DWBC appeared to cross the equator directly. Although the counter-current was clearly observed in both hemispheres, the degree of its connection between hemispheres could not be established from the data.

SOFAR floats give a more Lagrangian impression of the velocity field by providing point measurements following parcels of fluid along their paths (Richardson and Schmitz (1993)). Shown in Figure 1.5 is a summary of displacement vectors from float trajectories observed at 1800 meters in the Atlantic. It is this data set that also corresponds to velocity composition in Figure 1.3. Multiple trajectories are visible, but two characteristic paths stand out. One set of trajectories depicts floats travelling directly across the equator, at least to about $5^{\circ} \mathrm{S}$. The second shows an along-equatorial component, with floats being carried both east and west up to $1000 \mathrm{~km}$ along the equator. Similar to the tracer data, the floats suggest a bifurcation of fluid at the equator. 


\section{CFC's on $\sigma_{1.5}=34.63$}
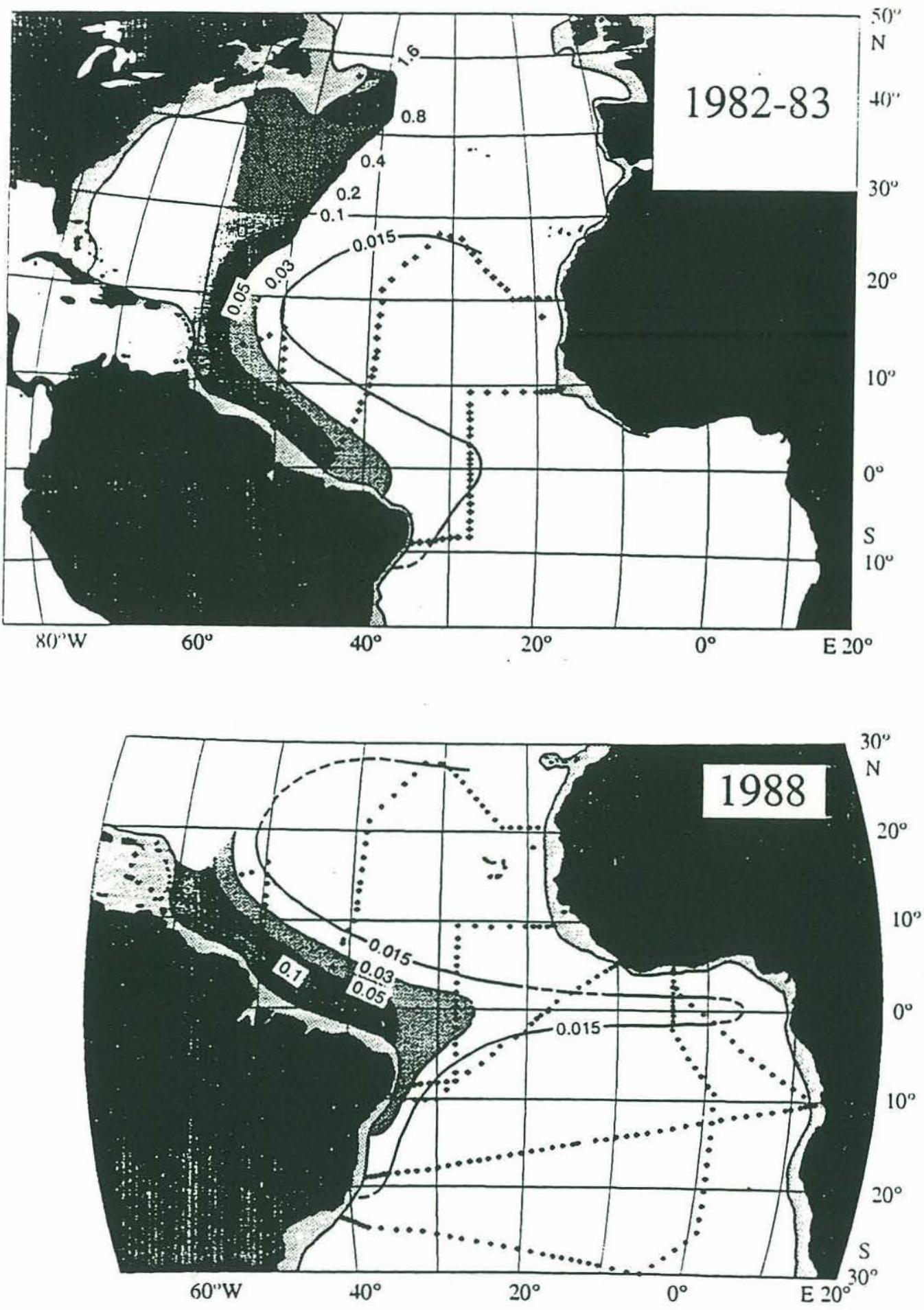

Figure 1.4: Composite distribution of $\mathrm{CCl}_{3} \mathrm{~F}$ in $\mathrm{pMol} \mathrm{Kg}^{-1}$ on the $\sigma_{1.5}=34.63 \mathrm{~kg} \mathrm{~m}^{-3}$ surface (roughly 1600 meters depth) in the Atlantic Ocean determined from a) TTO cruises (1981 and 1983) and b) five years later from SAVE expedition cruises and TTO measurements projected forward in time based on their apparent CFC ages. Figure courtesy of Ray Weiss. 


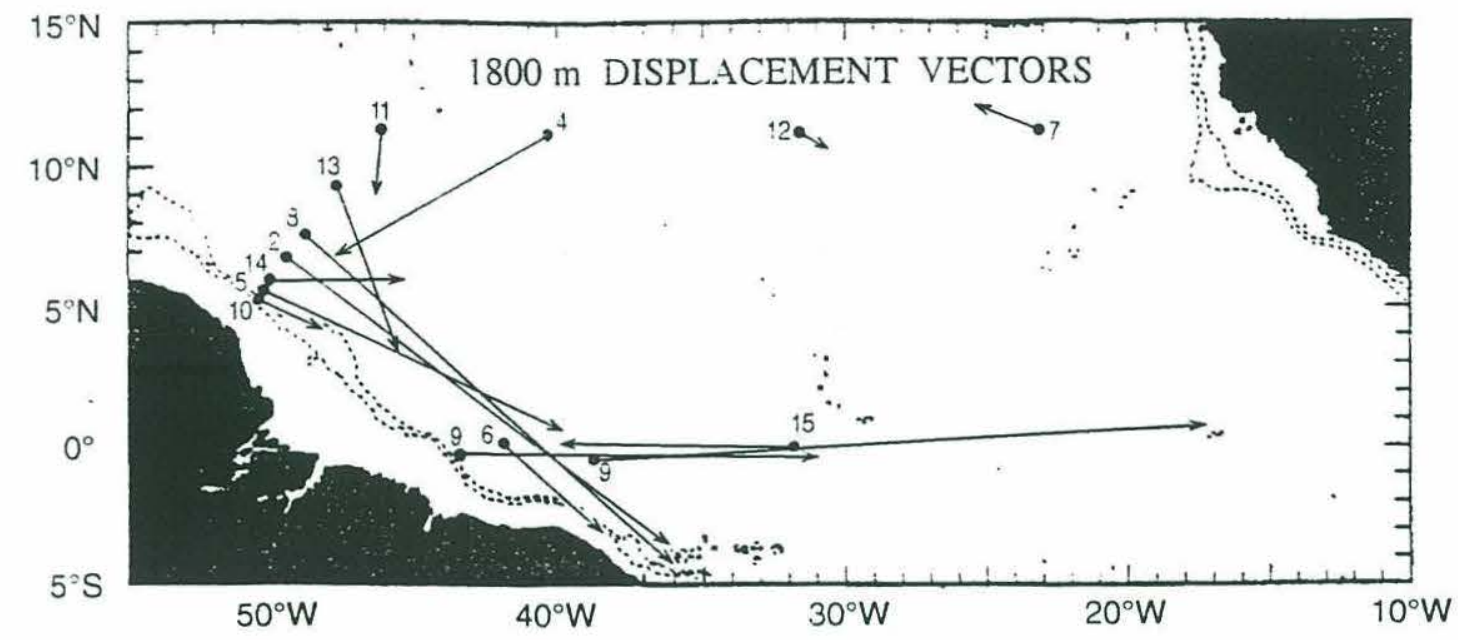

Figure 1.5: Displacement vectors of SOFAR float trajectories at 1800 meters depth between January 1989 and October 1990. Arrows indicate both along-boundary and along-equatorward motion. Figure adapted from Richardson and Schmitz (1993).

The variety of float trajectories in Figure 1.5 also indicates considerable variability in the region. The time-variability of the tropics has been noted in studies of the surface circulation particularly in the Pacific, but such behavior is characteristic of the tropical ocean at all depths. Johns et al. (1993) found 60-70 day fluctuations of the lower NADW boundary current properties from a current meter moored at $8^{\circ} \mathrm{N}$, $52^{\circ} \mathrm{W}$. Furthermore, additional SOFAR floats at 800 meters demonstrated loop-like behavior indicating sustained eddy-like features propagating in a northward-flowing intermediate-depth western boundary current. These eddies had a period varying between 7 and 40 days.

The data reflect the complicated, time-dependent circulation of the region. Even the mean path of fluid parcels in the tropical circulation is not well understood observationally. Although it seems likely that the fluid travels inter-hemispherically in a deep western boundary current, it is not clear whether the role of the equatorial filament is significant. In addition, it is not evident from these observations how fluid 
modifies its potential vorticity along its path.

\subsubsection{Dynamical}

Several dynamical studies of equatorial currents have contributed to the general background of nonlinear cross-equatorial flow. This research typically falls into two branches. As mentioned above, the Stommel and Arons (1960a) theory provided the initial theoretical framework for considering deep flow in the ocean, although it applies to currents somewhat weak to represent oceanic flows. One set of relevant dynamical studies emerges from this formulation.

Kawase (1987) extended the Stommel and Arons work by parameterizing the largescale upwelling, which had been prescribed in the earlier theory, so that the vertical velocity in the system increased with layer height deviation from an initial, resting value. The upwelling was still dependent on a mixing parameter, $\lambda$, which controlled the sensitivity of the upwelling to the height deviation. In so doing, this model obtained a steady-state equatorial tracer tongue for large values of $\lambda$, but the Stommel and Arons circulation resulted from small values of this parameter. Available data suggested that the value required for a tongue corresponds to unrealistically high values of the vertical mixing coefficient to represent features of low vertical mode. In addition, that work considered the time-dependent behavior of the spin-up. It showed that an along-equatorial mass flux occurs in the wake of an equatorial Kelvin wave resulting from a change in the source strength. Kawase concluded that the equatorial signal must result from time-dependent features of the circulation and not from a steady-state balance. The dynamics of this model were primarily linear.

A subsequent contribution by Kawase et al. (1992) showed the steady behavior of a full three-dimensional general circulation model for this problem. Forced by an imposed temperature anomaly at the northern boundary, the authors found again that the final state of the system consisted predominantly of a time-averaged, crossequatorial flow. Able to extend the system into a more nonlinear range than formerly, 
the study also demonstrated the development of time-dependent, eddy-like features in the boundary region.

Springer and Kawase (1993) explored the connection between a nonlinear western boundary and equatorial currents in an experiment designed to prevent the full spinup of the deep equatorial ocean. A sponge layer was placed at the eastern boundary to accept the initial Kelvin wave involved in the spin-up of the tropical ocean and prevent the mid-latitude interior from reaching the Stommel and Arons steady state. Thus the model reached a quasi-steady state that included a western boundary current in one hemisphere coupling to an along-equatorial meandering flow.

The second branch of relevant dynamical studies emerges from models of the subtropical gyre, and originates with the numerical demonstration by Bryan (1963) that the weakly nonlinear Munk boundary layer is unstable. More recent studies (e.g., Ierley (1987), Kamenkovich et al. (1995), and Sheremet et al. (1995)) have extended the study to include very nonlinear flows. These authors also find a break-down of the steady laminar Munk boundary layer and for particularly strong nonlinearity, the development of an inertial recirculation in one region of the basin. This recirculation is a persistent feature in the mid-latitude gyre, necessary to enhance the transport in the boundary layer to dissipate vorticity in the region. Although it is not necessary to change the sign of the potential vorticity in a mid-latitude model, it is necessary to balance vorticity input by the imposed wind-stress and reach a statistically steady state.

The instability of the Munk boundary layer at mid-latitudes has been examined through a linear stability analysis by Ierley and Young (1991) and Cessi and Ierley (1993). These studies demonstrate that the boundary layer is unstable to small perturbations at Reynolds numbers between 20 and 50, depending on the boundary conditions and model configuration. They define the instability as viscous because the trapped, growing modes result from the viscous term in their linear stability equation. 


\subsection{Outline of the Thesis}

This thesis examines the dynamics of western boundary currents constrained to cross the equator and is divided in principle into two portions. The first portion focusses on the central question of the thesis: how is the potential vorticity modified in a strongly nonlinear western boundary current to enable cross-equatorial flow? Chapters 2, 3, and 4 directly address this problem through the development of a numerical model and its diagnosis that shows that this process is carried out through the exchange of vorticity between frictional and turbulent boundary layers. The focus of Chapter 5 is more indirectly related to this question, discussing the cause of this dynamically essential eddy field.

Chapter 2 presents an overview of the model used in this thesis. It begins with a scaling of contributions to Ertel's potential vorticity which determines that the shallow-water model is the simplest representation of the full Navier-Stokes equations that adequately models cross-equatorial flow. A subsequent description outlines the shallow-water model itself and the configuration of the domain and forcing used in this study. The model is highly idealized with a rectangular domain and flat bottom, and it succesfully constrains fluid to cross the equator through a mass balance between a source in the northern hemisphere and a sink that is either distributed uniformly around the basin, or localized in the southern hemisphere. One critical non-dimensional parameter that determines the overall character of the system is the Reynolds number, which is defined as the ratio of the overall mass-flux through the system per unit depth to the viscosity. Finally, the numerical model is introduced with a brief description of its properties and necessary boundary conditions.

The numerical experiments for a range of Reynolds numbers are discussed in Chapter 3. At small Re, the system obeys linear dynamics and the connection to the Stommel and Arons (1960a) work is established. At a critical Re, the system becomes unstable with eddies developing immediately north of the equator, propagating southward and ultimately dissipating in the southern hemisphere. The sense of the 
circulation of these eddies follows from the vorticity of the fluid at the mass source. For still higher Reynolds numbers, the basin is filled with time-dependent features of both signs. Although instantaneous pictures of the height and velocity fields show a complicated pattern of interacting vortices, the time-mean picture reveals beneath the eddy field a well-defined western boundary current transporting fluid across the equator.

The diagnosis of these experiments is the subject of Chapter 4. After introducing the $\mathbf{J}$-vector as the primary tool to investigate the dynamics, examples of its application to the simple linear and weakly nonlinear systems illustrate analytically how vorticity fluxes through a basin strongly constrained by friction. Analysis of the strongly nonlinear experiments follows and shows the growth with Reynolds number of a turbulent boundary layer that transports vorticity between the inertial portions of the full boundary current and a frictional sublayer adjacent to the boundary where its transformation is clear. Thus the eddies have the essential dynamical role as the agents of vorticity modification that allows cross-equatorial flow. The chapter also dissects the time-mean boundary layer structure and includes a discussion of the sensitivity of the results to different boundary conditions and model configurations.

Chapter 5 examines the cause of the eddy field that emerges in the numerical model runs through a linear stability analysis of the western boundary current. Specifically three possible mechanisms are investigated, inviscid shear instability, viscous instability, and inertial instability. The formulation of the instability problem is followed by several sections on the instability under a range of conditions. Section 5.3.3, which considers geostrophic perturbations, examines the most idealized model and provides the simplest mathematical and physical description of the instability, finding that the western boundary current suffers a shear instability resulting from the horizontal variation in the potential vorticty structure. This section also illustrates that the instability is tropically enhanced because the maximum in the deformation radius occurs at the equator. 


\section{Chapter 2}

\section{The Model}

\subsection{Scaling Ertel's Potential Vorticity}

The dynamical problem of inertio-frictional cross-equatorial flow centers upon the balance between conservation and modification of potential vorticity. The dynamics of this flow could be studied with a range of models of varying complexity. However, as in other types of analysis, it is desirable to condense the necessary features of the full system into a simple form that focuses attention on those fundamental features. To determine the simplest model that contains the necessary physics to study crossequatorial flow, a comparison of the magnitude of different terms of the total potential vorticity highlights the components of the full momentum equations that govern inviscid flow. Ertel's potential vorticity, Equation 1.8, can be re-expressed in Cartesian coordinates and using subscripts to denote partial derivatives,

$$
P V=-\frac{1}{\rho}\left\{\left(w_{y}-v_{z}\right) \rho_{x}+\left(u_{z}-w_{x}+2 \Omega\right) \rho_{y}+\left(v_{x}-u_{y}+\beta y\right) \rho_{z}\right\} .
$$

The equatorial $\beta$-plane approximation in which $\beta=\frac{\partial(2 \Omega)}{\partial y}$ is the local gradient of planetary vorticity at the equator, $y=0$, has been used. The zonal, meridional, and vertical components of velocity are represented by $u, v$, and $w$, respectively. Although 
limited observations relating to cross-equatorial flow exist, it is possible to combine different measurements to estimate reasonable scales for the various components of Equation 2.1. As in the introduction, I approximate the along-boundary component of the flow to be meridional and take from Figure $1.3 \mathrm{~V}=50 \mathrm{~cm} \mathrm{~s}^{-1}$ and $\delta_{x}=100 \mathrm{~km}$. I assume that the vertical scale is $D=500$ meters and that the vertical velocity scales according to continuity: $W \leq \frac{U D}{\delta_{x}}$. Since the mean zonal velocity and meridional scale are not well determined by observations, I assume an upper limit on their values: $U=V$ and $\delta_{y}=\delta_{x}$. Density variations can be estimated to be $\rho_{y}=5 \times 10^{-7} \mathrm{~kg} / \mathrm{m}^{4}$ and $\rho_{z}=3 \times 10^{-4} \mathrm{~kg} / \mathrm{m}^{4}$ (McCartney (1993)). Given these values, all components of relative vorticity are approximately of the same scale, but the contributions of the horizontal components to the potential vorticity are small compared to that in the vertical. Furthermore, using $2 \Omega\left(=1.4 \times 10^{-4} \mathrm{~s}^{-1}\right)$ and $\beta=2 \times 10^{-11} \mathrm{~m}^{-1} \mathrm{~s}^{-1}$, the contribution by the horizontal component of planetary vorticity is also small in Equation 2.1 whereas that due to the vertical component can not be neglected.

Equation 2.1 then reduces to

$$
P V \cong-\frac{1}{\rho_{0}}\left\{\left(\beta y+v_{x}-u_{y}\right) \rho_{z}\right\}
$$

which corresponds directly to the shallow-water model. A single fluid layer contains the necessary physics to model observed cross-equatorial flows, neglecting interactions between layers. It is the difference between the internal compensation for changes in planetary vorticity through stretching versus that through changes in relative vorticity that makes the equator unique among latitudes, and the shallow-water model comprises both of these elements.

It is reasonable to inquire under what conditions the above scaling breaks down. For flows with strong meridional density variations, in a narrow band about the equator, the horizontal component of vorticity must be included in the dynamics. Naturally the shallow-water model is also insufficient to resolve flows with large vertical velocities. The use of the present model omits the possibility of density inversion. 


\subsection{The Shallow-Water Model}

The shallow-water model is a simplification of the full Navier-Stokes equations that describes the flow of a single layer of fluid, the active layer, generally bounded by a motionless layer of differing density, either above or below. Opposite the inactive layer is usually a solid boundary that contains the fluid with walls bounding the basin laterally. For a system in which vertical scales are small compared to horizontal scales and density variations within the fluid can be approximated to a narrow interface, the primitive equations of motion and continuity simplify to

$$
\begin{aligned}
\mathbf{u}_{t}+\mathbf{u} \cdot \nabla \mathbf{u}+f \hat{\mathbf{k}} \times \mathbf{u} & =-g / \nabla h+\mathbf{F} \\
h_{t}+\mathbf{u} \cdot \nabla h+h \nabla \cdot \mathbf{u} & =-w_{\star} .
\end{aligned}
$$

Here, $\mathbf{u}=(u, v)$ is the horizontal velocity vector, $g l=g \frac{\Delta \rho}{\rho}$ is the gravitational acceleration, reduced by the ratio of density variations at the interface to the density of the layer itself, and $h$ is the depth of the fluid. The forcing and dissipation are included in body force terms, $\mathbf{F}$, and in $w_{\star}$ which represents the cross-isopycnal mass flux between the active and inactive layers. The system as described in this thesis is laid out in Cartesian coordinates.

If the flow is strongly constrained by the Coriolis acceleration, velocities are approximately depth-independent. One measure of this strength is the Rossby number, $R o=\frac{U}{f L}$, which compares advective to planetary time scales. Mid-latitude currents away from the western boundary typically are characterized by a small Rossby number. This study, however, considers strong flows at low latitudes, and therefore the Rossby number is $\mathrm{O}(1)$. However, strong currents are still in cross-stream geostrophic balance even near the equator. Any vertical structure in the velocity field is neglected by the shallow-water equations.

The above equations relate to a homogeneous layer. The model is general in the sense that it can represent the full ocean as a single layer, in which case the bounding, 
inactive fluid is air, and $g /$ reduces to $g$. In this barotropic limit, layer depth variations are typically quite small since even small variations produce large pressure gradients and correspondingly large velocities. For cases in which the interface is internal as in this study, $g^{\prime} \ll g$, and the system is considered a $1 \frac{1}{2}$ layer model. The shallow-water model is also general in that it can represent an active layer sandwiched between two quiescent fields, as in Pedlosky (1987b). It is this configuration that best depicts the deep flows, far above the ocean bottom.

In nature, the ocean is driven by atmospheric forcing, by winds and by thermal and salinity variations, and dissipation is required to balance this input. The effect of winds is included in a shallow-water model through a wind-stress, $\tau$, in the momentum equations. Thermal and saline effects can be approximated by a single buoyancy forcing in the density equation of a full three-dimensional, baroclinic model. Through atmospheric forcing and small-scale mixing, fluid density is transformed from one value to another, resulting effectively in a cross-isopycnal mass flux. This influence is incorporated into the shallow-water system through a term, $B$, in the height equation. Most generally, dissipation is included in both the momentum equations, representing a large-scale viscosity, and in the height equation, as due to diffusion of density by vertical mixing.

With these additions, the equations take the form

$$
\begin{aligned}
\mathbf{u}_{t}+\mathbf{u} \cdot \nabla \mathbf{u}+f \hat{\mathbf{k}} \times \mathbf{u} & =-g / \nabla h+\tau-\text { visc } \\
h_{t}+\mathbf{u} \cdot \nabla h+h \nabla \cdot \mathbf{u} & =B-D i f f .
\end{aligned}
$$

Since the present study is directed toward deep flows in the ocean, the wind-stress is zero, and the vertical mass flux, B, is assumed to correspond to high-latitude forcing in regions where convection occurs. Thus, let

$$
B=B(x, y)
$$


where this function is assumed to be zero except in a small region near the northernmost latitude of the model. The function is constrained by a mass balance such that

$$
\frac{\partial}{\partial t} \iint h d A=\iint B d A-\iint D i f f d A
$$

which must be zero in the steady limit.

The dissipation in the real ocean is poorly understood, and several research groups are active in its study, from both observational and modeling perspectives. Rather than focussing on the intricacies that undoubtedly develop with different forms of dissipation and determining which form represents the most realistic, this study investigates only the simplest of parameterizations, with general conclusions drawn that may be useful when the subject of mixing is better understood. Viscosity is included as lateral friction. Specifically,

$$
\text { visc }=A_{H} \nabla^{2} \mathbf{u}
$$

and diffusion is a specified function of position,

$$
\operatorname{Diff}=\operatorname{Diff}(x, y) \text {. }
$$

Observations suggest that there is substantial spatial structure in the vertical mixing of the ocean. This structure seems to depend on the proximity of topographic features which enhance the internal wave field in its vicinity ( $J$. Toole (personal communication) and Kunze and Sanford (1996)). More than likely, it also depends on the internal dynamics of the system, such as the strength of the flow field and mass distribution. However, this dependence is difficult to estimate with any confidence. As a result, the mixing is assumed to be either uniform or localized. With buoyancy and mixing predetermined functions of location, the net mass flux in the model is specified entirely. However, the pathways by which fluid moves from the source at high latitudes to the sink is internally determined and this is the principle focus of the thesis.

No normal mass flux (no flow) conditions are applied at the lateral boundaries in all runs. No slip is used in most runs, with some attention paid briefly to free slip. 
Thus, on each boundary,

$$
\mathbf{u} \cdot \hat{n}=0
$$

and, either

$$
\mathbf{u} \cdot \hat{t}=0
$$

or

$$
\nabla \mathbf{u} \cdot \hat{n}=0
$$

where $\hat{n}$ and $\hat{t}$ are unit vectors normal and tangent to the boundary.

\subsection{Model Configuration and Scaling}

To study cross-equatorial flow, I configure a maximally simple geometry, a rectangular basin with vertical lateral boundaries, straddling the equator, on the equatorial $\beta$ plane. The basin extends a distance $L_{x}$ zonally, and $L_{y}$ meridionally, with $y=0$ corresponding to the central latitude as in Figure 2.1. Although the shallow-water equations can not distinguish between mass-fluxes through either an upper or lower interface - the dynamics of the vorticity equation respond only to vortex stretching not its location - the inflow has meaning with regard to the sign convention of the net mass flux, $w_{\star}$. As Equations 2.3a-2.3b are written and assuming the active layer rests beneath the inactive layer, a positive $w_{\star}$ corresponds to upwelling through the interface, and a negative value implies downwelling.

In this configuration, it is convenient to non-dimensionalize so that velocities are scaled by the gravity wave speed, $U=V=c_{0}=\sqrt{g^{\prime} H}$, horizontal lengths by the deformation radius, $\delta_{x}=\delta_{y}=L_{D}=\sqrt{\frac{c_{0}}{\beta}}, W=\frac{\hat{W}_{0}}{\beta L_{D} H}, A_{H}=\frac{\hat{A}_{H}}{\beta L_{D}^{3}}$, and the timescale, $T=\frac{1}{\beta L_{D}}$. Dimensional units are denoted with hats. With these definitions, the shallow water equations take the simple form

$$
\begin{aligned}
\mathbf{u}_{t}+\mathbf{u} \cdot \nabla \mathbf{u}+y \hat{\mathbf{k}} \times \mathbf{u} & =-\nabla h+A_{H} \nabla^{2} \mathbf{u} \\
h_{t}+\mathbf{u} \cdot \nabla h+h \nabla \cdot \mathbf{u} & =-W_{0} w_{\star} .
\end{aligned}
$$




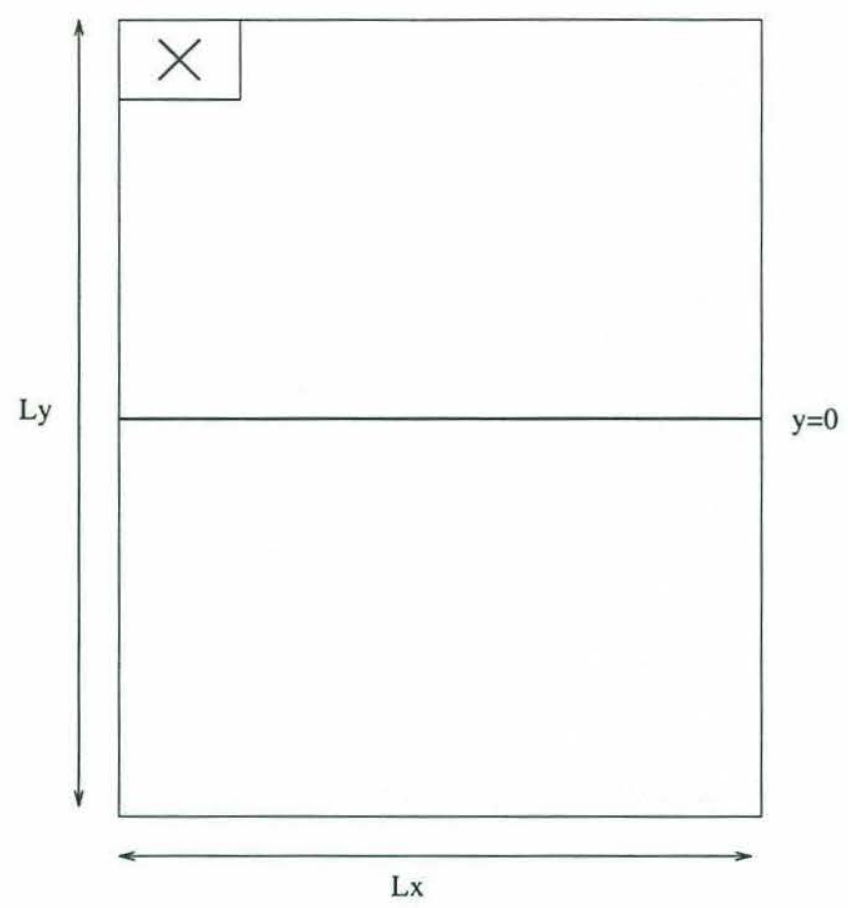

Figure 2.1: Schematic of the model under conditions of strong inflow in the northwest corner $(x)$ and weak uniform upwelling throughout the basin (shaded).

$W_{0}=\frac{S_{0}}{L_{x} L_{y}}$ represents the basin-wide average strength of the upwelling. Stated differently, $S_{0}$ is the source volume that is forced through the system, injected into the northwest corner, and removed elsewhere in the basin. $L_{x}$ and $L_{y}$ are the nondimensional basin dimensions. The volume flux, $S_{0}$, is scaled in units of $H c L_{D}$. For $H=500 \mathrm{~m}, c=1 \mathrm{~m} / \mathrm{s}$, and $L_{D}=250 \mathrm{~km}, S_{0}=0.2 H c L_{D}$ corresponds to an actual transport of $25 \mathrm{~Sv}$.

With this non-dimensionalization, the four external parameters of the original system, $\beta, g^{\prime}, \hat{A}_{H}$, and $\hat{W}_{0}$, conveniently reduce to two, the forcing and dissipation. The range of model behavior is more easily spanned.

Introducing the the top-hat function,

$$
\Pi(x)= \begin{cases}1 & \text { if }|x|<\frac{1}{2} \\ 0 & \text { if }|x| \geq \frac{1}{2}\end{cases}
$$


the forcing used in most experiments of the thesis has the form

$$
w_{\star}=-\frac{L_{x} L_{y}}{\Delta x \Delta y} \Pi\left(\frac{x-x 0}{\Delta x}\right) \Pi\left(\frac{y-y 0}{\Delta y}\right)+1 .
$$

Additional experiments use more localized upwelling,

$$
w_{\star}=-\frac{L_{x} L_{y}}{\Delta x \Delta y}\left(\Pi\left(\frac{x-x 0}{\Delta x}\right) \Pi\left(\frac{y-y 0}{\Delta y}\right)-\Pi\left(\frac{x-x 1}{\Delta x}\right) \Pi\left(\frac{y-y 1}{\Delta y}\right)\right) .
$$

Parameters, $x 0, x 1$, etc. shift the central location of the source and sink, and the

dimensions $\Delta x$ and $\Delta y$ give the widths of those regions. Referring to experiments with the former mass-flux distribution as the "uniform" upwelling case is a partial misnomer since the mass source is also included in $w_{\star}$. However, since the interior flow is determined by the local vorticity balance (i.e., the local upwelling), it is convenient hereafter to distinguish the two forcing distributions as "uniform" (Equation 2.14) and "non-uniform" or "localized" (Equation 2.15). An example of the localized upwelling configuration is given in Section 4.2, where this distribution is applied.

\subsection{The Vorticity Equation and Reynolds Num- ber}

Although two parameters, $W_{0}$ and $A_{H}$, govern Equations $2.12 \mathrm{a}-2.12 \mathrm{~b}$, the dynamics may be simplified further by considering a single parameter. To this end it is useful to derive the vorticity equation, obtained by taking the curl of the shallow-water momentum equations:

$$
\frac{\partial \zeta}{\partial t}+\mathbf{u} \cdot \nabla \zeta+v+(\zeta+y) \nabla \cdot \mathbf{u}=A_{H} \nabla^{2} \zeta
$$

As in the introduction, boundary layer scales can be estimated assuming different steady-state balances in Equation 2.16. For example, a balance of planetary vorticity advection and vorticity diffusion gives the Munk boundary layer scale,

$$
\delta_{M}=A_{H}^{\frac{1}{3}} .
$$


The balance of relative and planetary vorticity advection leads to the inertial boundary layer scale,

$$
\delta_{I}=\sqrt{U}
$$

where $U$ is the scale of the zonal velocity within the boundary layer. The forcing parameter, $S_{0}$, relates to the inertial boundary layer scale by setting the magnitude of the zonal current to which the layer matches. Under conditions of uniform forcing, for example,

$$
\delta_{I}=\sqrt{\frac{2 S_{0}}{H L_{y}}} .
$$

As a result, the two parameter system of forcing and dissipation can be restated in terms of Munk and inertial boundary layer scales.

An additional length scale is derived assuming a balance of nonlinearity and friction in Equation 2.16. This inertio-frictional scale is defined

$$
\delta_{M}^{\star}=\sqrt{\frac{\delta_{M}}{\delta_{I}}} \delta_{M}
$$

This balance arises when the full boundary layer structure is predominantly inertial. Even for a very small viscosity, friction must ultimately become significant in the balance at some scale, though one smaller than the Munk scale.

It is convenient to define the Reynolds number, which represents the relative strength of nonlinearity and dissipation. The ratio of these two terms in Equation 2.16 gives

$$
R e_{1}=\frac{U \delta}{A_{H}}
$$

where $\delta$ represents the zonal scale over which vorticity variations occur. Taking $\delta=\delta_{I}$ as for example in Kamenkovich et al. (1995) gives

$$
R e_{1}=\left(\frac{\delta_{I}}{\delta_{M}}\right)^{3} .
$$

Although this quantity is related to the external parameters of the system through Equations 2.17 and 2.19, the definition of the inertial boundary layer width remains undesirably dependent on the distribution of the upwelling in the interior. 
A second measure of relative strengths of nonlinearity and dissipation can be defined as the ratio of the source strength per unit depth to the viscosity:

$$
R e_{2}=\frac{S_{0}}{A_{H} H} .
$$

I prefer the second measure, which follows Ierley and Young (1991), since it is unambiguously defined by the input parameters. Future references to the Reynolds number will drop the subscript, 2.

\subsection{The Numerical Model}

\subsubsection{Spatial Discretization}

To study the system numerically, Equations $2.12 \mathrm{a}$ and $2.12 \mathrm{~b}$ must be discretized, and several choices are available. I employ a modified version of the potential enstrophy conserving model of Sadourny (1975) for this study. In this model a numerical form of the mass-weighted square of the potential vorticity is approximately conserved. A comparison to a similar energy conserving model showed that in the inviscid limit a numerical scheme that does not conserve potential enstrophy results in the unphysical growth of rotational energy at small scales, leading to unrealistic flows and unrealistic energy dissipation. Potential enstrophy conservation avoids this small scale catastrophe. Another useful model is the potential enstrophy and energy conserving finite-difference scheme of Arakawa and Lamb (1978), described in Haltiner and Williams (1980). With more conserved quantities, this scheme potentially produces flows that are more consistent with the continuous system. However, the model is more complicated and therefore more difficult to diagnose accurately. The Sadourny model has the advantage that it is relatively simple and slightly faster to run. I have examined both models and qualitative comparisons of the output show good agreement in the gross behavior of the system. The inclusion of dissipation in this work, even though quite weak, renders the conservation properties of the model less vital 


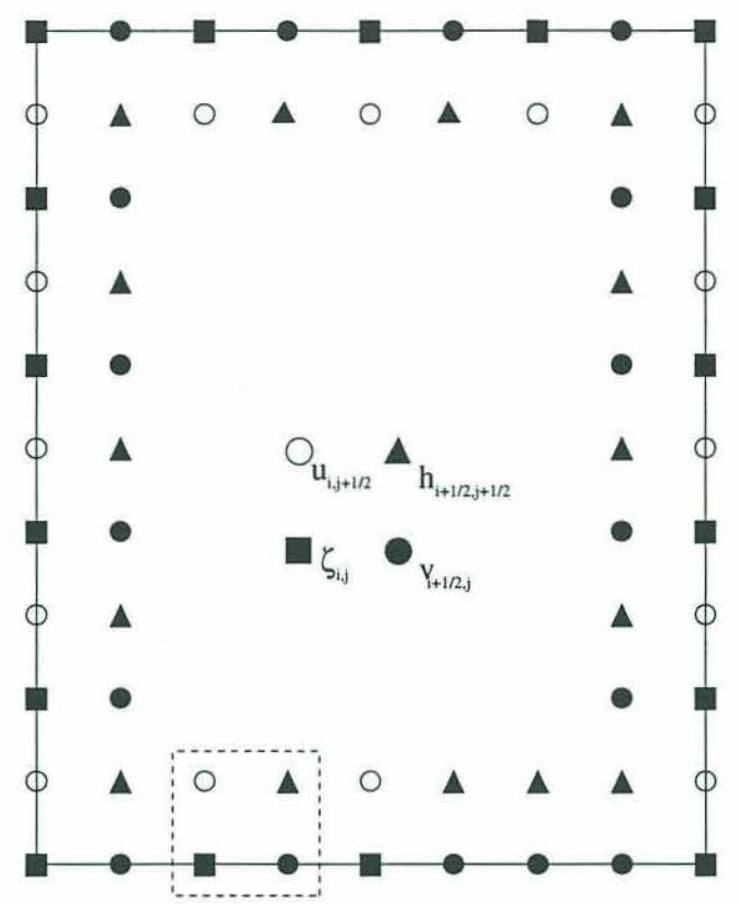

Figure 2.2: Schematic of the grid used in the model.

than in inviscid studies.

The numerical model is laid out on a C-grid, on which dependent variables are staggered. Consider the basin divided up into a series of cells of uniform dimension, as in Figure 2.2 with layer depth, $h$, at their centers. Vorticity and potential vorticity lie at cell corners, and velocities, $u$ and $v$, are directed perpendicular to and located at the midpoint of the bounding line segments of each cell.

Notationally, varibles are defined $\zeta_{i, j}, u_{i, j+\frac{1}{2}}, v_{i+\frac{1}{2}, j}$, and $h_{i+\frac{1}{2}, j+\frac{1}{2}}$, where $i$ represents the zonal index and $j$, the meridional. It is convenient to create averaging and differencing operators, defined at midpoints of connecting line segments, and a Laplacian operator, defined at the point at which it operates. For example, in the case of relative vorticity, $\zeta$, let

$$
\begin{aligned}
\bar{\zeta}_{i+\frac{1}{2}, j}^{x} & =\frac{1}{2}\left(\zeta_{i, j}+\zeta_{i+1, j}\right) \\
\delta_{x} \zeta_{i+\frac{1}{2}, j} & =\frac{1}{\Delta x}\left(\zeta_{i+1, j}-\zeta_{i, j}\right)
\end{aligned}
$$


and similarly in $y$, and

$$
\Delta^{2} \zeta_{i, j}=\delta_{x}\left(\delta_{x} \zeta_{i, j}\right)+\delta_{y}\left(\delta_{y} \zeta_{i, j}\right)
$$

$\Delta x$ is the grid spacing in the $x$-direction. It can be shown the differential and averaging operators commute with themselves, so that multiple operations may be taken in any order.

Using this notation, the discretization takes the form

$$
\begin{aligned}
u_{t}-\bar{q}^{y} \bar{V}^{x y}+\delta_{x} \phi & =A_{H} \Delta^{2} u \\
v_{t}+\bar{q}^{x} \bar{U}^{x y}+\delta_{y} \phi & =A_{H} \Delta^{2} v \\
h_{t}+\delta_{x} U+\delta_{y} V & =-w_{\star},
\end{aligned}
$$

where the potential vorticity is defined

$$
q=\frac{f+\zeta}{\bar{h}^{x y}}
$$

and

$$
\begin{aligned}
\zeta & =\delta_{x} v-\delta_{y} u \\
U & =\bar{h}^{x} u \\
V & =\bar{h}^{y} v \\
\phi & =g^{\prime} h+\frac{1}{2}\left({\overline{u^{2}}}^{x}+{\overline{v^{2}}}^{y}\right) .
\end{aligned}
$$

As in Adcroft (1995), several rules can be determined from the operators 2.24a2.24c. Letting $k$ represent either horizontal direction, and variables $\psi$ and $\eta$ be general,

$$
\begin{aligned}
& \delta_{k}(\psi \eta)=\bar{\psi}^{k} \delta_{k} \eta+\bar{\eta}^{k} \delta_{k} \psi \\
& \delta_{k}\left(\bar{\psi}^{k} \eta\right)=\psi \delta_{k} \eta+{\overline{\eta \delta_{k} \psi}}^{k}
\end{aligned}
$$

and the square of the geometric mean of a variable is

$$
\langle\psi\rangle_{k+\frac{1}{2}}^{2}=\psi_{k} \psi_{k+1}
$$


The potential enstrophy of the continuous system is defined, $\xi=h q^{2}$, and the following equation can be derived from the discretization $2.25 \mathrm{a}-2.25 \mathrm{c}$ with $A_{H}=w_{\star}=0$,

$$
\frac{\partial}{\partial t}\left(q^{2} \bar{h}^{x y}\right)+\delta_{x}\left(l q l_{x}^{2} \bar{U}^{x y}\right)+\delta_{y}\left(l q l_{y}^{2} \bar{V}^{x y}\right)=0 .
$$

Thus an approximate form of the potential enstrophy is conserved.

Details of the numerical model time-stepping and grid-stretching can be found in Appendix A.

\subsubsection{Numerical Boundary Condition}

The boundary conditions for the continuous system consist of no normal flow through the solid boundaries, and no slip or free slip at the basin walls. However, the discretization, Equations $2.25 \mathrm{a}-2.25 \mathrm{c}$, requires the specification of the potential vorticity at all grid points on the boundary as well as-within the domain, and therefore an additional boundary condition is required to step forward the model. Although the no flow, no/free slip conditions determine the absolute vorticity on the boundary, they do not determine the layer height. This must be determined separately, but it is desired to be consistent with the continuous system.

Consider the zonal momentum equation,

$$
\frac{D u}{D t}-f v=-g^{\prime} \frac{\partial h}{\partial x}+A_{H} \nabla^{2} u,
$$

where $\frac{D}{D t}$ represents the material derivative. Setting $u=0$ as is true at meridional boundaries and rearranging terms leaves

$$
\frac{\partial h}{\partial x}=\frac{1}{g^{\prime}}\left(f v-A_{H} \frac{\partial^{2} u}{\partial x^{2}}\right)
$$

The meridional velocity is determined by the no/free slip condition, but the curvature of the zonal velocity is not. I choose the simplest, though somewhat arbitrary, boundary condition that sets the gradient of the curvature to zero, namely

$$
\frac{\partial^{3} u}{\partial x^{3}}=0
$$


at the meridional boundaries, and similarly in $v$ at the zonal boundaries.

A second boundary condition that may be viable would be to step forward the height equation at the boundary,

$$
\frac{D h}{D t}+h \nabla \cdot \mathbf{u}=-W_{0} w_{\star}
$$

Although I have not implemented this form for the boundary condition, I can assess one measure of consistency between the chosen condition with the one that is exactly consistent with the continuous system. If the two conditions were inconsistent, then the model would not conserve mass. This represents an integrated, rather than local check, but I have compared mass starting an integration and mass after 3000 time units, and found the two to match exactly.

\subsubsection{Parallel Programming}

Although the model is general and has run on various serial platforms, it has been optimized for use on a massively parallel computer, the Thinking Machine CM-5, that runs at MIT as part of an ARPA supercomputer initiative, project SCOUT (contract \#MDA972-92-J-1032). This particular machine has 128 nodes, each with a single SPARC microprocessor, 4 specialized vector units and $32 \mathrm{MB}$ of memory. The routine has also run on a 32 node machine at NCAR.

Massively Parallel computers such as the CM-5 consist of several processing units with distributed memory. The whole system is interconnected by a high-speed communications network. Each processing unit can operate independently, but total processing power does not increase linearly with the number of processing elements. The speed of operations depends on the efficiency with which information is supplied to each processor. As a result, different types of communications are divided by efficiency into a hierarchy operations with varying desirability. Even with a high-speed network, passing information between distant nodes is considerably slower than accessing nearest neighbor information, which in turn is slower than reading local memory units. 
Thus the optimization of the CM5 consists of coding the routine such that it minimizes all inter-processor communications.

The shallow water model is well-suited to parallel computation because it is inherently local. Each processor represents a grid element, and the time stepping of each variable depends only on local information or local derivatives which are nearest neighbor operations. Some effort is required to insure that the layout of the many variables are aligned properly so that local information is indeed stored locally. When the number of grid elements exceeds the number of processing units, the CM5 compiler creates virtual units that automatically optimize local information transfers. 


\section{Chapter 3}

\section{Linear to Nonlinear}

\section{Cross-equatorial Flow}

\subsection{Introduction}

The numerical experiments comprising this study and using different model parameters are summarized in Table 3.1. The experiments primarily consider upwelling to be uniform following Equation 2.14 with a few runs devoted to the case with upwelling localized at high latitudes as in Equation 2.15. The linear run is checked against the analytic solution, and then comparisons are made with more strongly forced experiments. Higher Reynolds numbers are achieved both by increasing the forcing and keeping dissipation constant and by maintaining forcing and reducing the frictional boundary layer width. An upper limit on the forcing is that which produces horizontal velocities approaching the gravity wave speed. Model runs in which this bound is exceeded fail when the variable $h$ vanishes in the vicinity of the equator where the rotational constraint is weak, and thickness advection can be large due to nonlinear steepening. Some comparison is made between the runs using higher forcing values and those using weaker dissipation. 


\begin{tabular}{|c|c|c|c|c|c|c|c|c|}
\hline Run & $S_{0}$ & $A_{H}$ & $H_{0}$ & $\delta_{M}$ & $\delta_{I}$ & $R e_{1}$ & $R e_{2}$ & Comments \\
\hline U1 & .01 & .001 & 2 & 0.1 & 0.022 & 0.011 & 5 & \\
\hline U2 & .04 & .001 & 2 & 0.1 & 0.045 & 0.089 & 20 & \\
\hline U2.A & .032 & .001 & 1 & 0.1 & 0.056 & 0.18 & 32 & \\
\hline U3 & .1 & .001 & 2 & 0.1 & 0.07 & 0.35 & 50 & \\
\hline U4 & .01 & .0002 & 1 & 0.058 & 0.032 & 0.16 & 50 & \\
\hline U5.X & .3 & .001 & 2 & 0.1 & 0.12 & 1.84 & 150 & $L_{x}=15$ \\
\hline U5.Y & .3 & .001 & 2 & 0.1 & 0.12 & 1.84 & 150 & $L_{y}=30$ \\
\hline U6 & .2 & .0005 & 2 & 0.079 & 0.1 & 2.0 & 200 & \\
\hline U7 & .2 & .00029 & 2 & 0.065 & 0.1 & 3.5 & 350 & \\
\hline U8 & .1 & .0002 & 1 & 0.05 & 0.1 & 5.0 & 500 & \\
\hline U9 & .05 & .00005 & 1 & 0.037 & 0.07 & 7.07 & 1000 & $\delta x_{\min }=0.0054$ \\
\hline UFS & .2 & .0005 & 1 & 0.1 & 0.1 & 1.0 & 200 & Free Slip \\
\hline N2 & .1 & .00067 & 1 & 0.087 & 0.07 & 0.52 & 75 & \\
\hline UBar & $10^{-4}$ & $10^{-6}$ & 1 & 0.01 & 0.014 & 2.82 & 100 & \\
\hline
\end{tabular}

Table 3.1: Table of numerical runs for uniform upwelling configuration. All experiments use no slip boundaries except for that noted. $H_{0}$ refers to the initial undisturbed depth of the layer.

\subsection{Spin-up of the Equatorial Ocean}

Before examining the steady-state solution it is useful to consider the spin-up of the system from a state of rest. The evolution of the flow in this type of equatorial ocean has been well-documented by Kawase (1987). That work used linear momentum dissipation rather than the Laplacian form used here, but this difference is minor in the limit of weak flows. Linear wave dynamics describe this evolution well.

Illustrated in Figure 3.1 is a series of instantaneous portraits of the height and velocity fields at three times after $t=0$, when the forcing is initiated. The run is frictional and forced weakly, with $S_{0}=0.01, A_{H}=10^{-3}$, and $H=1$. Fluid entering into the source region in the north-west corner of the basin forms a small mound which begins geostrophic adjustment within a rotation period. An anti-cyclonic gyre surrounding the source forms along with a Kelvin wave that travels southward along the western boundary. In its wake follows the fluid entering at the source. Upon reaching the equator, this wave couples into an eastward propagating equatorial 
Kelvin wave. Although the mass source is located in the northern hemisphere, the development of the wave field is nearly symmetric about the equator soon after the signal reaches the equator. At the eastern boundary, the disturbance splits into two oppositely directed Kelvin waves travelling along that boundary. Finally, anomalies at the eastern boundary accompanying the passage of each Kelvin wave excite Rossby waves which transmit this information westward across the interior. By establishing a steady resupply of fluid from the western boundary current, the passage of the Rossby wave train arrests the continued drainage of the interior that results from the upwelling in that region. Although a spectrum of Rossby waves is generated by the Kelvin wave at the eastern boundary, in practice it is soon after the passage of the fastest mode that the interior reaches an approximately steady state. At the western boundary, a steady current forms, consistent with the frictional parameterization. In the present study, there forms a southward flowing Munk boundary current with a weak off-boundary counterflow.

The final state of the system includes an interior flow that is consistent with the linear theory of Stommel and Arons (1960a). With uniform upwelling, the interior flow is weakly poleward and eastward. There is no equatorial signal. Fluid entering the source-region merely follows the western boundary to the latitude where it breaks from the coast and migrates slowly into the interior, ultimately to be upwelled out of the basin.

In the separate case of a localized, southern hemisphere sink, there is no interior flow in the final state. Two intense high-latitude gyres remain, one anti-cyclonic for inflow, and one cyclonic for outflow, connected by the boundary current.

The development of this flow occurs in two phases, the initial Kelvin wave propagation around the basin boundary, and the subsequent Rossby waves which return information across the interior. The group velocities for these waves indicate the time-scale for spin-up. 

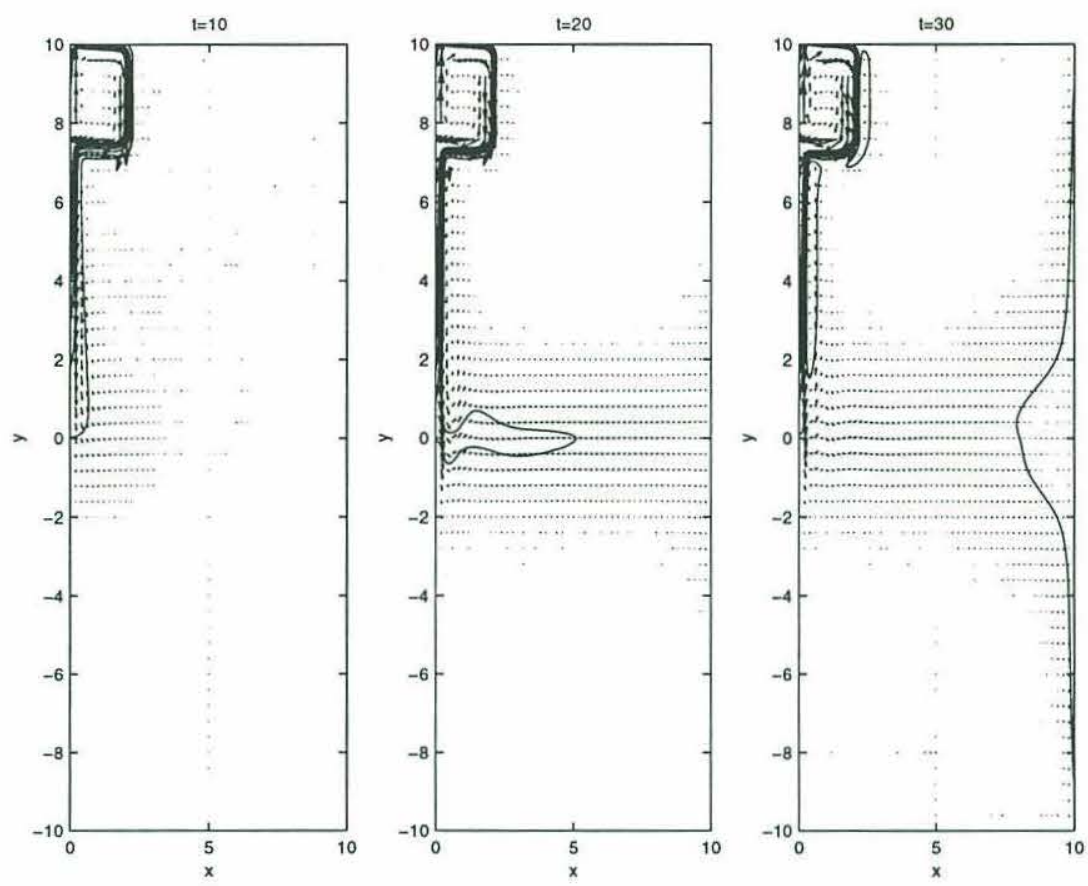

Figure 3.1: Time sequence of height contours and velocities for a case of linear spinup. The mean height is 1 , with contours every 0.002 . The initial spinup is marked by the passage of a Kelvin wave along the western boundary, the equator, and the eastern boundary.

The Kelvin waves, which move at the gravity wave group speed of

$$
c_{g}^{K}=\sqrt{g^{\prime} H},
$$

travel about the basin a distance $L=L_{y}+L_{x}$. Thus the time scale for the boundary field is

$$
\begin{aligned}
T^{K} & =\frac{L}{c_{g}^{K}} \\
& =30
\end{aligned}
$$

for the standard run of this study. Figure 3.1c shows a snapshot at this time for a weakly forced run.

The Rossby wave time scale is estimated from the dispersion relation

$$
\omega=-\frac{\beta k}{k^{2}+l^{2}+\frac{f_{0}^{2}}{c^{2}}} .
$$


The fastest westward-propagating mode is that for which horizontal wavenumbers, $k$ and $l$, are zero (i.e., long waves). In this case, the group velocity is given by

$$
\begin{aligned}
c_{g}^{R} & =-\frac{\beta}{\frac{f_{0}^{2}}{c^{2}}} \\
& =-\frac{1}{y^{2}}
\end{aligned}
$$

in the scaling of this model. The time scale for Rossby waves, which is strongly latitude dependent, is considerably longer than for Kelvin waves. With a maximum latitude of 10 deformation radii, the time scale for adjustment of the interior is approximately

$$
\begin{aligned}
T^{R} & =\frac{L_{x}}{c_{g}^{R}} \\
& =1000
\end{aligned}
$$

Thus the time-scale for Rossby wave propagation is significantly longer than that for the Kelvin waves and determines the required time for the linear system to reach steady-state.

One measure of the degree to which the system has reached equilibrium is to compare height fields. Figure 3.2 shows the difference in height field between times 1000 and 1200 for the linear run, U1. In this case the weak upwelling over the interior is $w_{\star}=5 \times 10^{-5}$. Thus $w_{\star} \Delta t=10^{-2}$. The variation in height fields, with a magnitude of $10^{-6}$, has some structure associated with the slow high latitude Rossby waves and very weak equatorial waves, but the system is very near to a steady state.

The spin-up of a strongly forced run is not considerably different from the case described above. A series of plots presented in Figure 3.3 show the sequence. The initial descent of the Kelvin wave along the western boundary is similar, and it couples into an equatorial mode, just as in the linear case. However, the group velocity comprises both the gravity wave speed and current advection (Philander (1990)). Furthermore, the coupling is somewhat more complicated. There exists an inertial overshoot of the Kelvin wave (Springer and Kawase (1993)), and associated with that cross-equatorial flow is an instability. As a result, a time dependent region in the vicinity of the western 


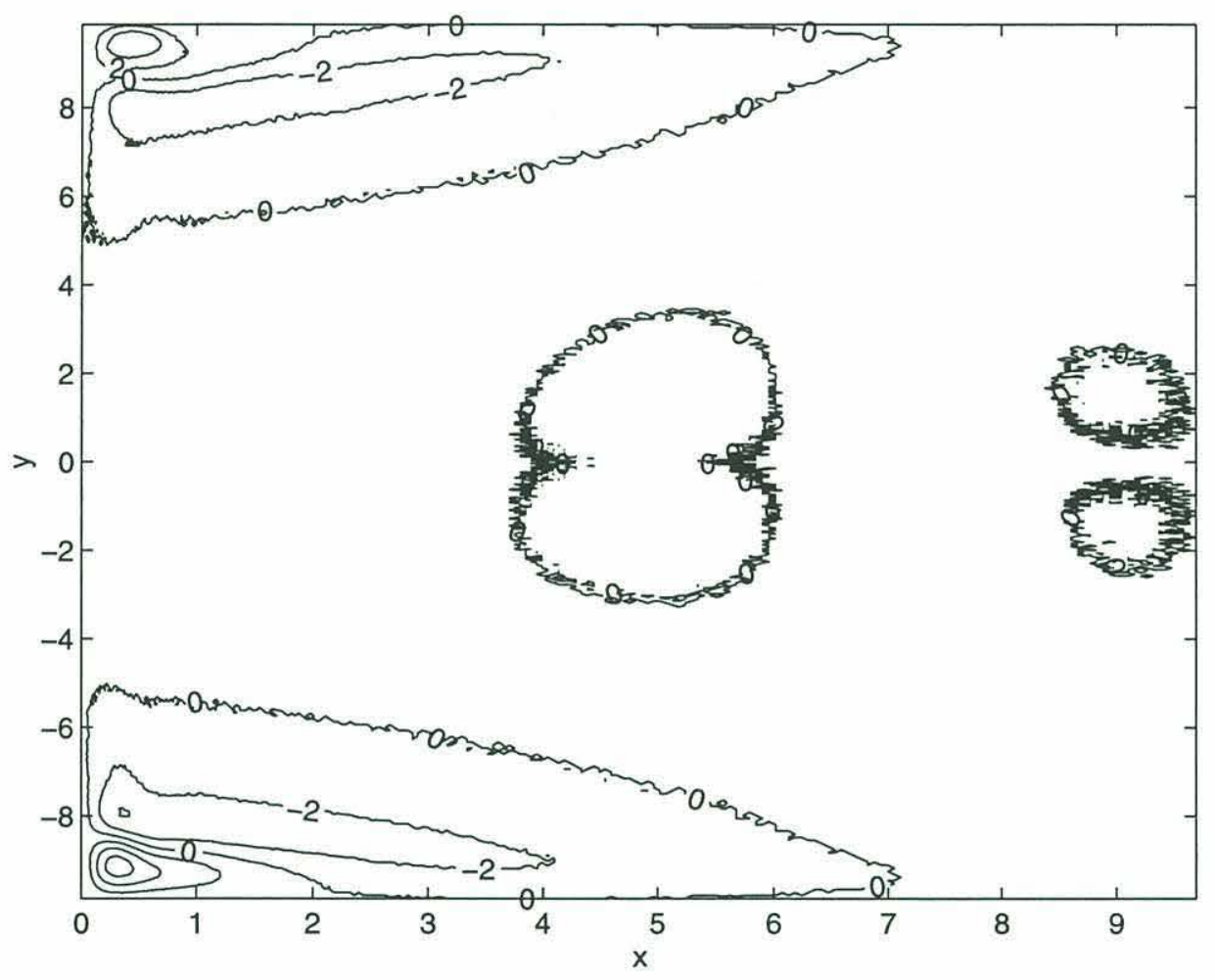

Figure 3.2: Difference in height of the linear model run U1 between time $t=1000$ and $t=1200$ $\left(\times 10^{6}\right)$. Small structure at high latitudes shows that the system has not reached steady state, but is near. 
boundary and equator remains behind, even after the Kelvin wave has passed. The subsequent behavior of the system is similar, with the time-scale to reach a now quasisteady state largely determined by that of the Rossby waves in the interior. Figure 3.4 shows the difference between mean height fields at time $t=1100$ and $t=3000$ for run U7. Fields are averaged over 100 time units to remove highest frequency fluctuations. In this case, $w_{\star} \Delta t \cong 1$, and the average height differences of the interior are a fraction of this amount. Only near the dynamically active western boundary does the height difference appear. In this region, low frequency fluctuations remain and intense features shift slightly in the domain over long time periods. In addition, this region is strongly dominated by advective processes which have a much shorter time-scale than the basin waves. As in the linear case the system has reached a quasi-steady state. All time-averaging in the measurements in this study begin at time $t=1000$ except run U9 (which begins at $t=700$ ) in which the zonal basin dimension is only 5 and therefore the spin-up is faster. A check of the sensitivity to this value for the very nonlinear run (U8) shows very minor quantitative differences in averaged quantities that begin at time $t=2000$.

The time dependence is transmitted throughout the interior, again via equatorial and boundary Kelvin waves, and Rossby waves, but the height variations are small compared to the larger anomaly of the initial pulse. That the effect of nonlinearity is not more strongly felt in the interior is a testament to the degree to which linear physics describe fields away from the western boundary current and the equator.

\subsection{Linear Solution and Numerical Experiment}

The linear solution, which can be determined from a combination of Stommel and Arons (1960a) theory with a Munk (1950) boundary layer, provides a useful baseline to compare the more complicated nonlinear flow. This section reviews these two theories using the no slip boundary condition only. 

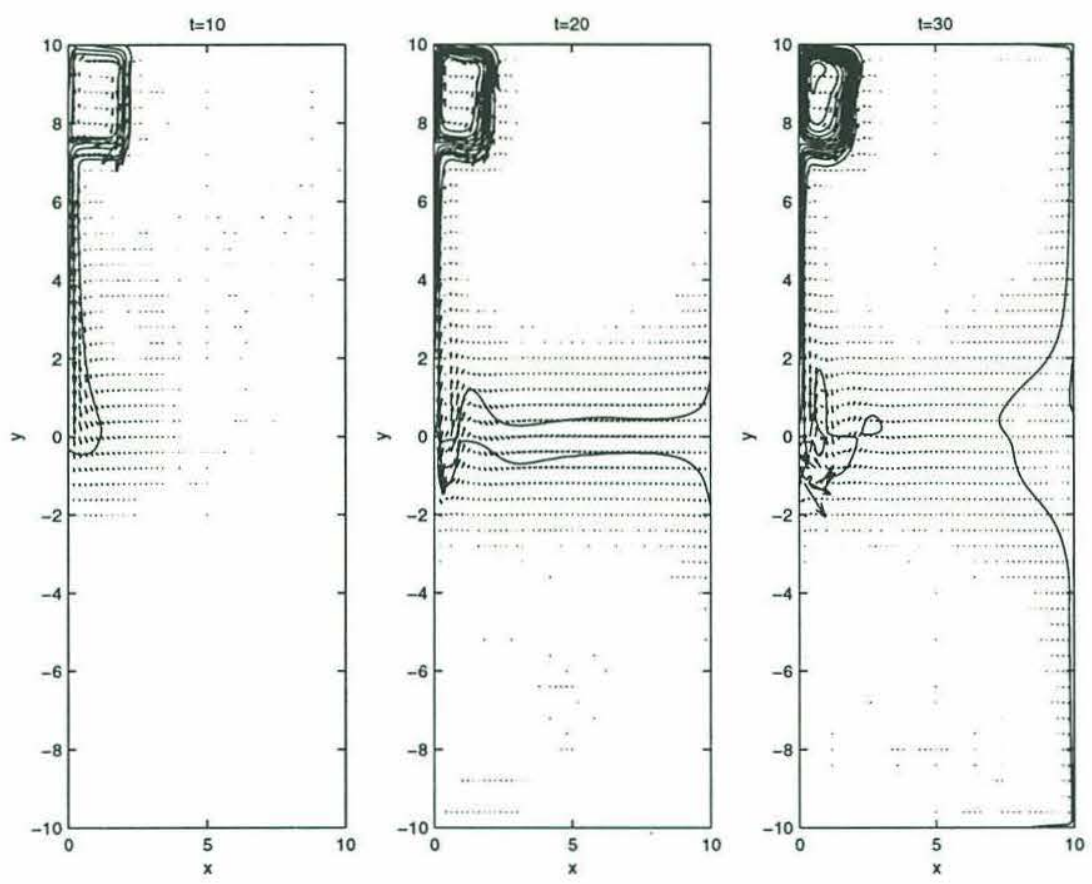

Figure 3.3: Time sequence of height contours and velocities for a case of a nonlinear spinup. The undisturbed height is 1 , with contours every 0.1 . The same sequence as in the linear case is noted, with the addition of remaining variability at the intersection of the western boundary with the equator. The Reynolds number for this run is 1000 . 


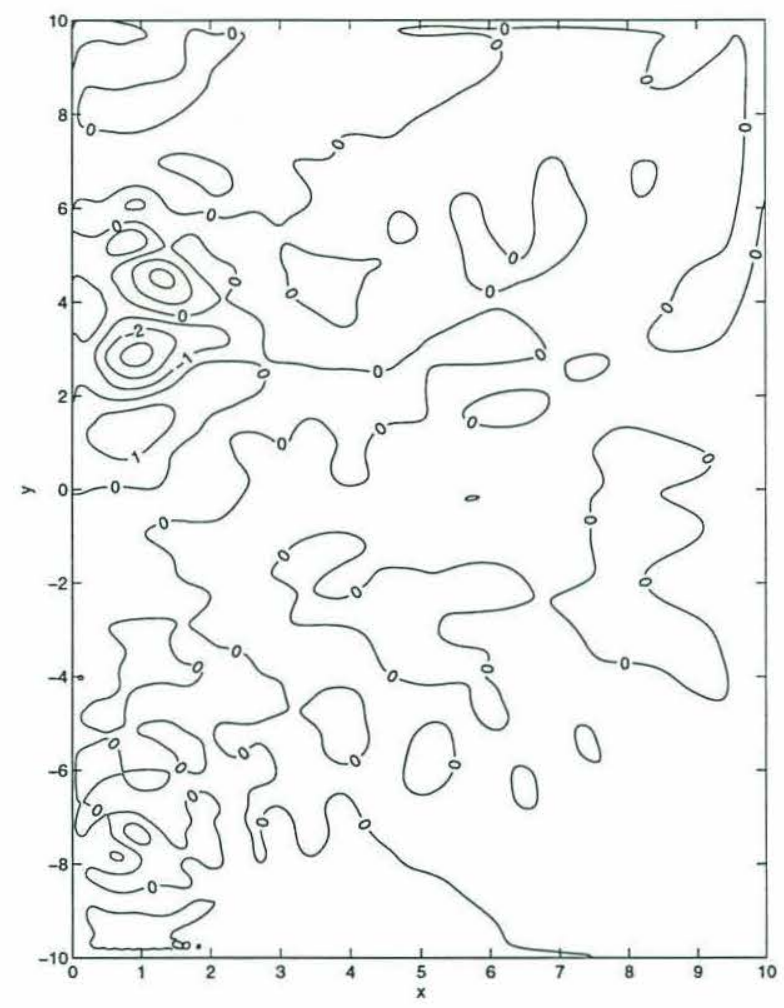

Figure 3.4: Difference in 100 time unit averaged height fields of the nonlinear model run U7 between time $t=1100$ and $t=3000(\times 10)$. Some structure remains at the western boundary where low frequency motions shift large features, but the system has reached a quasi-steady state. 
Assuming weak, steady currents and small layer height variations the equatorial $\beta$-plane shallow-water equations reduce to

$$
\begin{aligned}
-\beta y v & =-g \prime \frac{\partial \eta}{\partial x} \\
\beta y u & =-g \prime \frac{\partial \eta}{\partial y}+A_{H} \frac{\partial^{2} v}{\partial x^{2}} \\
\nabla \cdot \mathbf{u} & =-\frac{w_{\star}}{H},
\end{aligned}
$$

where $h=H+\eta$, and $\eta \ll H$. The assumption that intense boundary currents exist only at the western edge of the basin (which results from the existence of nonzero $\beta$ ) has been made implicitly in the one component of viscosity that remains in the equations. The meridional velocity is in geostrophic balance.

The linear vorticity equation, which follows directly from Equations 3.5a-3.5c, expresses a balance of planetary advection of vorticity with stretching and vorticity diffusion:

$$
\beta v=\beta y \frac{w_{\star}}{H}+A_{H} \frac{\partial^{3} v}{\partial x^{3}} .
$$

No slip and no flux boundary conditions apply along the basin boundary:

$$
v=0 ; u=0
$$

It is convenient to separate variables into interior and boundary layer components,

$$
u=u_{I}+u_{B} ; \quad v=v_{I}+v_{B} ; \quad \eta=\eta_{I}+\eta_{B} .
$$

Taking the Munk boundary layer parameter, $\delta_{M} \ll L_{x}$, the interior fields are determined to be

$$
\begin{aligned}
& v_{I}=y \frac{w_{\star}}{H} \\
& u_{I}=\int_{x}^{x_{e}}\left[\frac{2 w_{\star}}{H}+\frac{y}{H} \frac{\partial w_{\star}}{\partial y}\right] d x \\
& h_{I}=H-\int_{x}^{x_{e}} \frac{\beta y^{2} w_{\star}}{g^{\prime} H} d x
\end{aligned}
$$


The boundary layer structure results from a balance of planetary vorticity advection and vorticity diffusion. This balance is expressed in terms of the layer height variable, $\eta_{B}$,

$$
\frac{\partial \eta_{B}}{\partial x}=\delta_{M}^{3} \frac{\partial^{4} \eta_{B}}{\partial x^{4}}
$$

Thus

$$
\eta_{B}=\left[C_{1} \cos \left(\frac{\sqrt{3}}{2 \delta_{M}} x\right)+C_{2} \sin \left(\frac{\sqrt{3}}{2 \delta_{M}} x\right)\right] e^{-x / 2 \delta_{M}}+C_{3} e^{x / \delta_{M}}+C_{4} .
$$

Variables, $C_{i}$, are functions of latitude, $y$. That the boundary layer variables must vanish as $\frac{x}{\delta_{M}}$ becomes $\mathrm{O}\left(L_{x}\right)$ requires $C_{3}=C_{4}=0$. The no slip condition sets to leading order

$$
C_{2}=\frac{1}{\sqrt{3}} C_{1} \text {. }
$$

The no flow condition determines $C_{2}$ by integrating the height equation, Equation 3.5c, over a sub-area, $A$, of the basin, bounded to the north, east, and west by basin boundaries, and to the south by a line of latitude, as in Figure 3.5. Given no flux and no slip boundary conditions, this integrated equation reduces to

$$
\int_{0}^{x_{E}} v d x=\iint_{A} \frac{w_{\star}}{H} d x d y
$$

and simply restates the conservation of mass within the domain, $A$.

Inserting the geostrophic relation for $v$ and separating interior and boundary layer components gives

$$
C_{1}=\eta_{B}(0, y)=-\frac{\beta y}{g^{\prime} H} \int_{0}^{x_{E}}\left[\int_{y}^{y_{N}} w_{\star} d y-y w_{\star}\right] .
$$

Horizontal velocities for the boundary layer, $u_{B}$ and $v_{B}$, follow directly from the governing equations.

$$
\begin{aligned}
& v_{B}=\frac{g^{\prime}}{\beta y} \frac{\partial \eta_{B}}{\partial x} \\
& u_{B}=-\frac{g^{\prime}}{\beta y} \frac{\partial \eta_{B}}{\partial y}+\frac{\partial^{2} v_{B}}{\partial x^{2}} .
\end{aligned}
$$




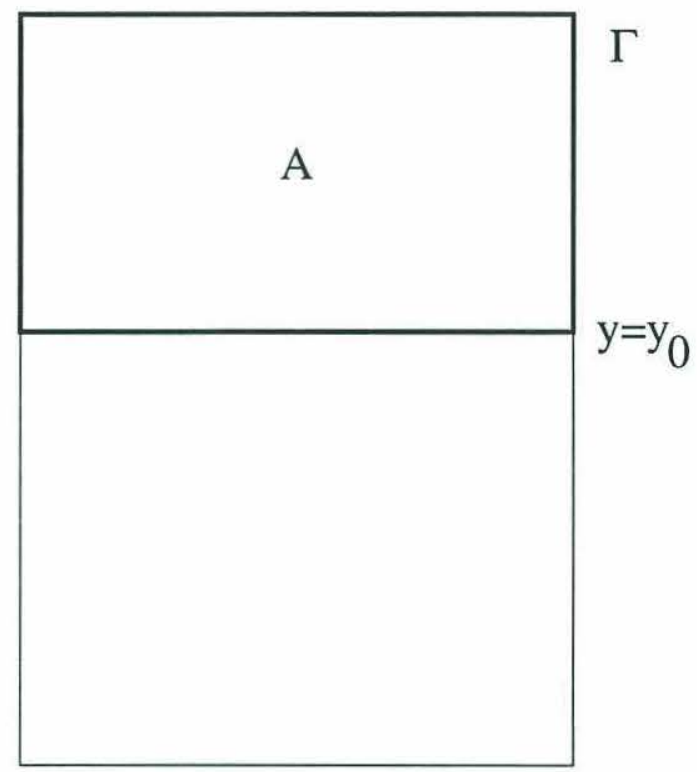

Figure 3.5: Subdomain A of full basin bounded by a border $\Gamma$ comprising the eastern, western, and northern boundaries, as well as a line of latitude at $y=y_{0}$.

Although the fields depend strongly on the distribution of the forcing, $w_{\star}$, some features remain robust. The interior is locally forced, with the meridional velocity linearly proportional to the local upwelling. The zonal interior velocity field acts to distribute mass longitudinally to accommodate the upwelling and meridional velocity. The interior height field simply reflects a geostrophic balance with the velocity fields.

It is helpful to consider the simplest of cases, in which the upwelling is constant, $w_{\star}=W_{0}$, and there exists a source of fluid having a mass flux, $S_{0}$, entering through the northern boundary. The dynamics associated with the mass flux will be ignored, except in that it provides the necessary mass to the system to be expelled through the layer interface. Under this assumption,

$$
\begin{aligned}
& v_{I}=y \frac{W_{0}}{H} \\
& u_{I}=\frac{2 W_{0} l_{x}}{H} \\
& h_{I}=H-\frac{\beta y^{2} W_{0} l_{x}}{g^{\prime} H}
\end{aligned}
$$


where, $l_{x}=x_{E}-x$, and

$$
C_{1}=\frac{\beta y}{g^{\prime} H}\left[W_{0} L_{x}\left(2 y-y_{N}\right)-S_{0}\right]
$$

As before $L_{x}$ is the full zonal scale of the basin. This case is particularly simple, and both the height field and the horizontal velocities are illustrated in Figure 3.6. Parameters for this plot are $L_{x}=10, y_{N}=10, W_{0}=5 \times 10^{-5}, S_{0}=-W_{0} L_{x} L_{y}$, $g^{\prime}=\beta=1$ and the undisturbed height is $2 \mathrm{H}$ The Munk boundary layer width, $\delta_{M}=0.1$. Height contours are every $0.25 \mathrm{H}$ except for $1.9<h<2.1$, where the interval is $0.025 \mathrm{H}$. This figure shows the classic Stommel and Arons interior solution with a Munk boundary layer. The interior has weak poleward and eastward flow in both hemispheres. The velocity vectors are naturally small in regions where the flow is weak and difficult to distinguish in the figure. In these regions, the sense of the flow must be inferred from the height contours. There is an intense western boundary current that brings fluid south from its entrance into the basin at the northern boundary. There appears to be considerable flux through the northern and southern boundaries, both in the interior and the western boundary. It is possible to extend the above calculations to include northern and southern boundary currents, but these features are not particularly illuminating, except that they transfer mass back from the interior of the basin to the western boundaries.

One characteristic feature of the Stommel and Arons (1960a) theory is that the transport of the boundary current vanishes north of the southern boundary of the basin. In the figure, it is apparent that this occurs at $y=-5$, and this latitude can be calculated from the mass balance of the interior flow. Consider a basin whose northern boundary coincides with the equator and whose source is located at this northern edge. The mass balance at any line of latitude yields

$$
\int_{0}^{x_{E}}\left(v_{I}+v_{B}\right) d x=\int_{y_{S}}^{y} \int_{0}^{x_{E}} \frac{w_{\star}}{H} d x d y
$$

Inserting Equation 3.9a and setting the boundary layer transport to zero determines 


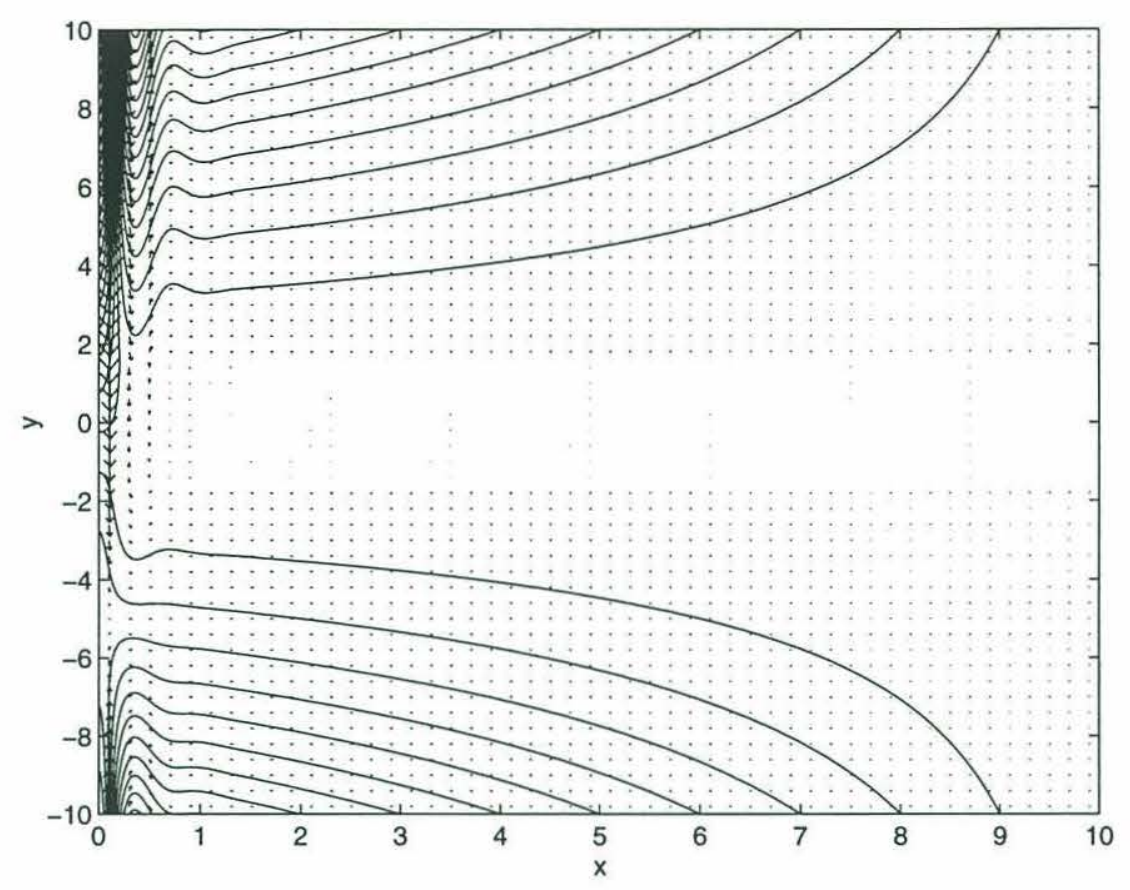

Figure 3.6: Steady solution to Equation 3.6 with uniform upwelling and a Munk boundary layer width of 0.1 .

the critical latitude. In the case when upwelling is uniform,

$$
y_{c}=\frac{1}{2} y_{S} .
$$

When upwelling is localized as a step function south of latitude, $y_{1}$,

$$
y_{c}=\frac{1}{2}\left(y_{S}-y_{1}\right) .
$$

In the current example, $y_{S}=-10$ and $y_{1}=0$ which gives the appropriate value for $y_{c}$. This location divides the boundary current into two halves, one driven by the existence of the northern source feeding the basin upwelling, and a second current, driven by the local upwelling of the southern hemisphere, independent of the source in the north. Fluid destined to reach the southern portion of the basin near the western boundary has an extensive journey from the western boundary in the north through the interior in the south and back to the western boundary before exiting the basin.

The time-dependent numerical model has been run to test the code in this linear limit. Shown in Figure 3.7 are the height contours and horizontal velocities of a 


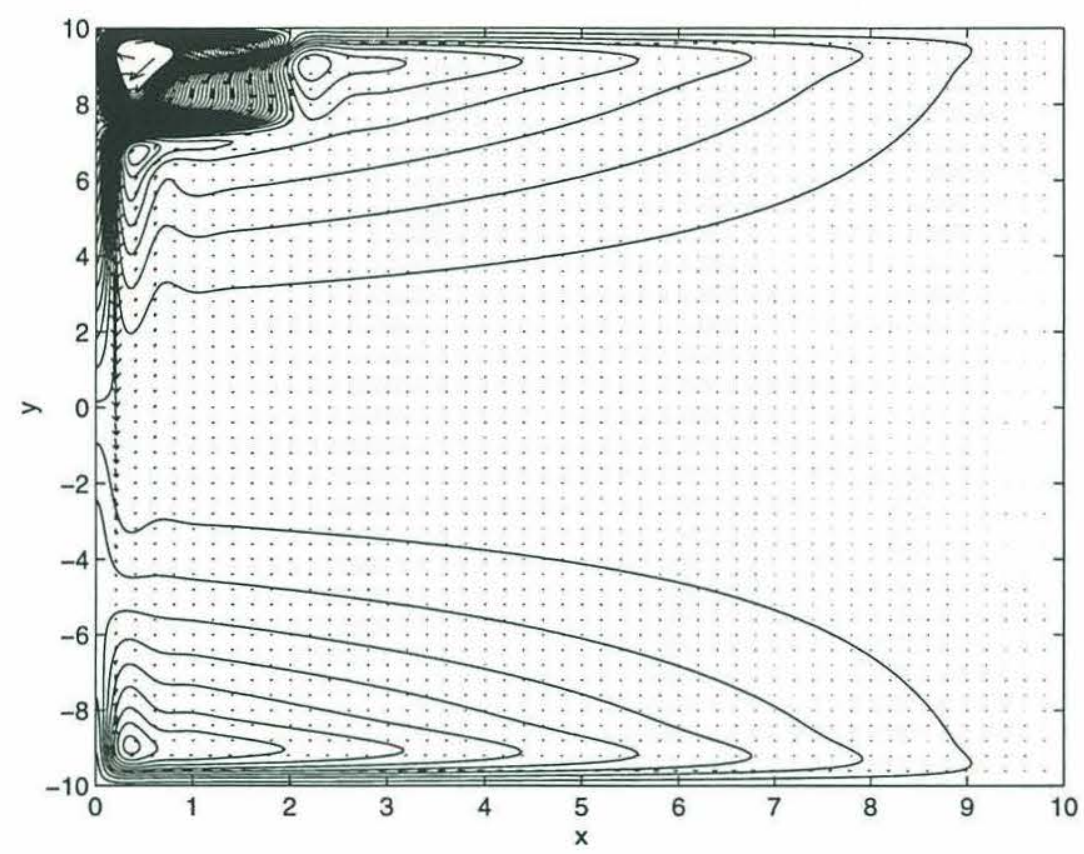

Figure 3.7: Steady state achieved by the numerical model, using uniform upwelling, except for the source region in the north west corner. Compare to Figure 3.6. Note that the Reynolds number for this run is $R e=5$.

numerical model run, U1, that corresponds directly to that of the analytic calculation above. In the north-western corner of the basin is a region of large velocities and height gradients. This is the source region for fluid in the numerical model, and its dynamics are not included in the theory presented above. Also visible are the northern and southern boundary currents that return mass to the western boundary. The contours in both figures are identical and can be compared directly. The numerical and analytically calculated fields match well, far from the region of strong forcing and the northern and southern boundaries.

As expected, the system divides into multiple gyres, one very small and associated with the localized source of fluid to the system and two hemisphere-scale gyres divided by the equator. In this linear limit, the potential vorticity contours, not shown, simply follow lines of latitude. 


\subsection{Transition to Instability}

As the Reynolds number increases, the steady linear solution becomes deformed by the influence of nonlinearity in the dynamics. The interior remains largely linear in its balance, but inertial effects become important in the boundary layer. As the meridional velocity of the boundary current increases, the relative vorticity in that region becomes non-negligible. The qualitative distinction from the linear solution is that the potential vorticity contours now detour from $f$-contours near the western boundary. When the boundary condition is no slip, the PV contours shift northward just at the boundary. When the condition is free slip, the contours shift only slightly at

the boundary, the result of small variations in the layer height, but more significantly, they extend south of the lines of latitude just off the boundary.

At a critical Reynolds number, $20<R e_{c}<50$, the system becomes unstable, and a steady state solution is no longer achieved by the time-dependent model. However, a quasi-steady state is reached. Instantaneous pictures of the height and velocity fields show the development of periodic eddies just north of the equator in the western boundary current. An example from Run U3 with $R e=50$ is presented in Figure 3.8. Only 50 vectors, linearly interpolated from the stretched onto the regular grid shown, span the basin in the figure. However, approximately 50 grid points in the model successfully resolve eddies in this region.

Formed north of the equator, these eddies are always cyclonic, reflecting the high potential vorticity of their source fluid relative to the local planetary vorticity. The eddies migrate coherently southward along the boundary, dissipating vorticity along their way by creating anomalously large velocity gradients in the vicinity of the boundary. As the eddies propagate across the equator their vorticity decreases until they join smoothly with the steady southern hemisphere flow to the south.

This state of the flow is time-dependent, but still only weakly nonlinear. The system has a periodicity associated with these features, and their geographic extent is extremely limited, one eddy length scale east into the basin, and a few deformation 


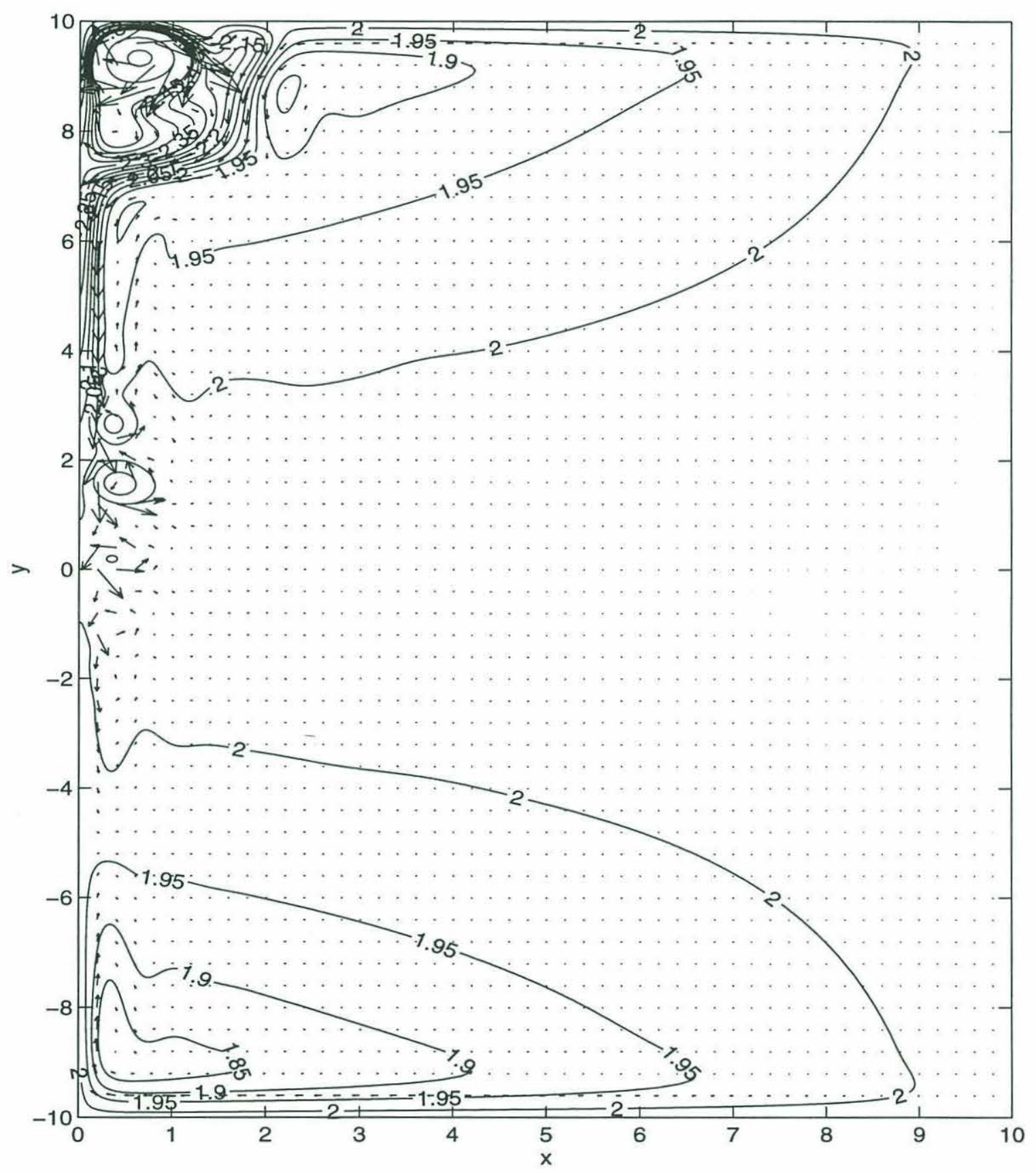

Figure 3.8: Instantaneous height contours and velocity vectors for run, U3. $R e=50$. 
radii north and south of the equator. The eddy motion is very regular and time-series analyses (not shown) of the potential vorticity at various locations in the basin indicate periodic structure. The time-scale associated with the eddies is $\mathrm{O}\left(20\left(\beta L_{D}\right)^{-1}\right)$, which corresponds to $\mathrm{O}(40$ days $)$ in dimensional units. This time-scale can be adjusted by tuning parameters in the model, but realistic scalings for the transport and layer depth yield time-scales that compare sensibly with observations of variability as in Richardson and Schmitz (1993) or Johns et al. (1993). A more detailed discussion of the eddy generation process is found in Chapter 5.

Additional information can be gained by considering time-averaged fields. Figure 3.9 shows the height contours and velocity field for the basin averaged over 2000 time units of the model $\left(\mathrm{O}(100)\right.$ eddy time-scales) after spin-up at $t=1000 \beta^{-1} L_{D}^{-1}$. In the mean, these fields look quite similar to the linear solution. The height contours are deformed somewhat and the potential vorticity contours are more exaggerated at the western boundary, but overall there is little qualitative difference.

\subsection{Strongly Nonlinear Flow}

At still larger Reynolds number, the influence of nonlinearity becomes ever stronger. The eddies grow more intense and strongly deform mean potential vorticity contours. The height contours extend farther to the south. The geographic extent of the variability extends over a broader portion of the basin, both meridionally and zonally along the equator.

Figure 3.10 shows the instantaneous height and velocity fields for a run at $R e=500$ at time, $t=2900$. The eddy field is extremely disordered, populating a broad swath of the western part of the basin. Eddies extend south of the critical latitude, $y_{c}$, where boundary currents meet. There is a clear indication of wave propagation out along the equator and eastern boundary, and some variability is visible in the interior as deformations of the height contours in that region. The existence of such strong 


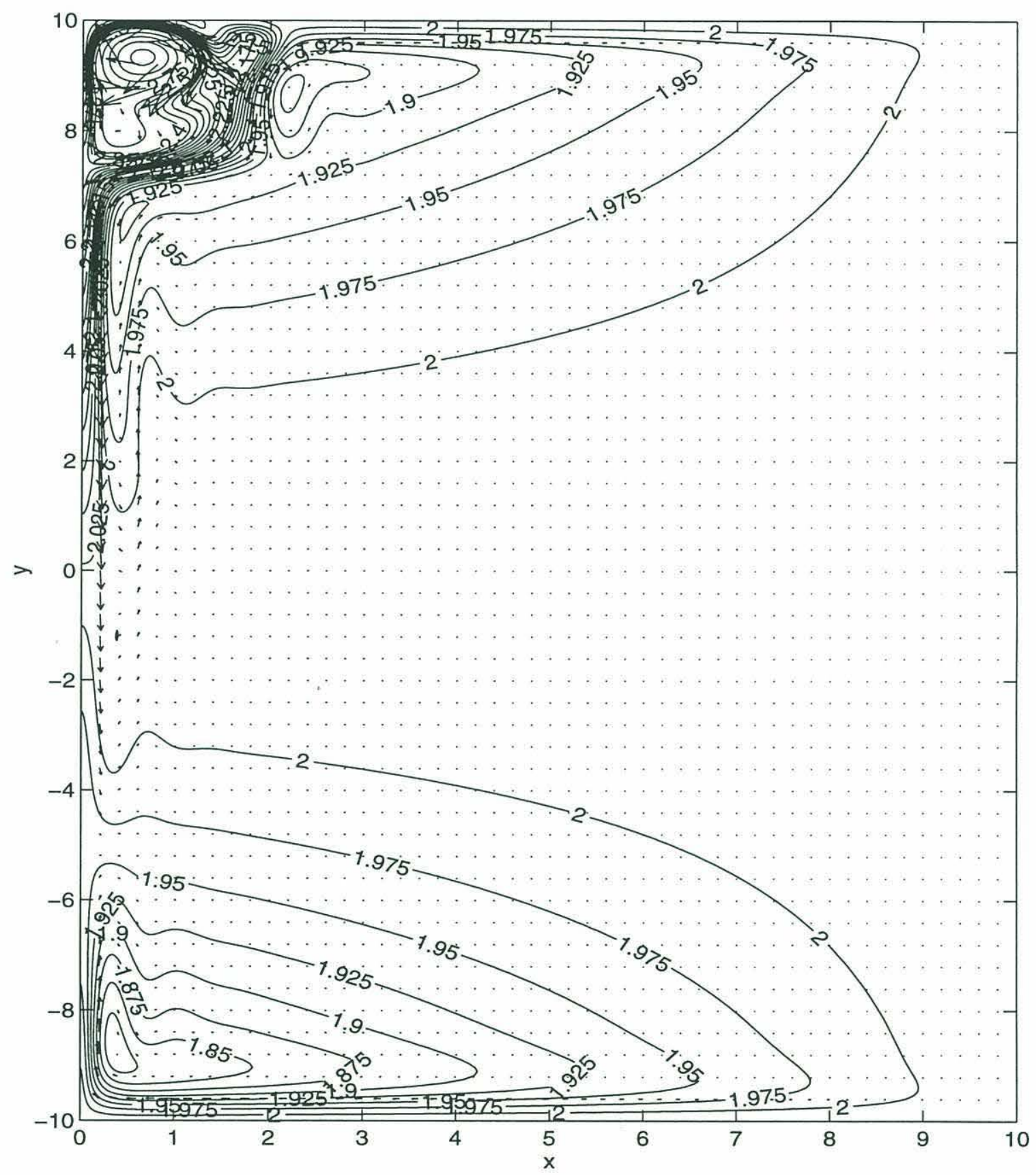

Figure 3.9: Time averaged height contours and velocity vectors for run, U3. $R e=50$. 
flows near the equator suggests a possible mechanism to generate the tracer tongue of chloroflouromethane observed by Weiss et al. (1985) and shown in Figure 1.4. A calculation of the time-mean velocity (shown below) reveals a meandering current near the equator and the western boundary that weakens quickly eastward. More likely responsible for the tongue is the Stokes drift associated with the time-varying, equatorial waves visible in Figure 3.10. This possibility was first suggested by Kawase and Sarmiento (1986) and seems plausible given the strong generation of equatorial waves in this model.

The instantaneous potential vorticity field provides a similarly complicated illustration of the anomalies in this highly nonlinear run. This field is presented in Figure 3.11 which is shaded to aid the distinction between positive (light) and negative (dark) values. Direct correlation between strong features in the two figures is evident in isolated regions of anomalous potential vorticity. These regions have various dimensions, though a few structures near the western boundary have a common scale of about 1 deformation radius. Eddies of both sign are now created in the formation process and are found in either hemisphere, though generally not far from the equator. In addition, there exist large gradients in the potential vorticity field, particularly near the coherent eddy of the northern hemisphere and near the western boundary. Indeed it is not difficult to imagine that these large gradients are crucial in the process of potential vorticity transformation as will be seen in the next chapter.

Also visible in Figure 3.11 is the potential vorticity of the source fluid. Since this quantity depends on the dynamical behavior of the source region it is not easily related to the input parameters of the system and must be diagnosed from the results. The source fluid in this experiment has a value of potential vorticity between 4 and $6\left(\beta L_{D} H\right)^{-1}$, though the fluid in the narrow western boundary current has a range of values. In the northern hemisphere, negative potential vorticity is found immediately adjacent to the boundary because of the extreme shear, and the zonal gradient is very large, with potential vorticities of magnitude $6\left(\beta L_{D} H\right)^{-1}$ just a fraction of a 


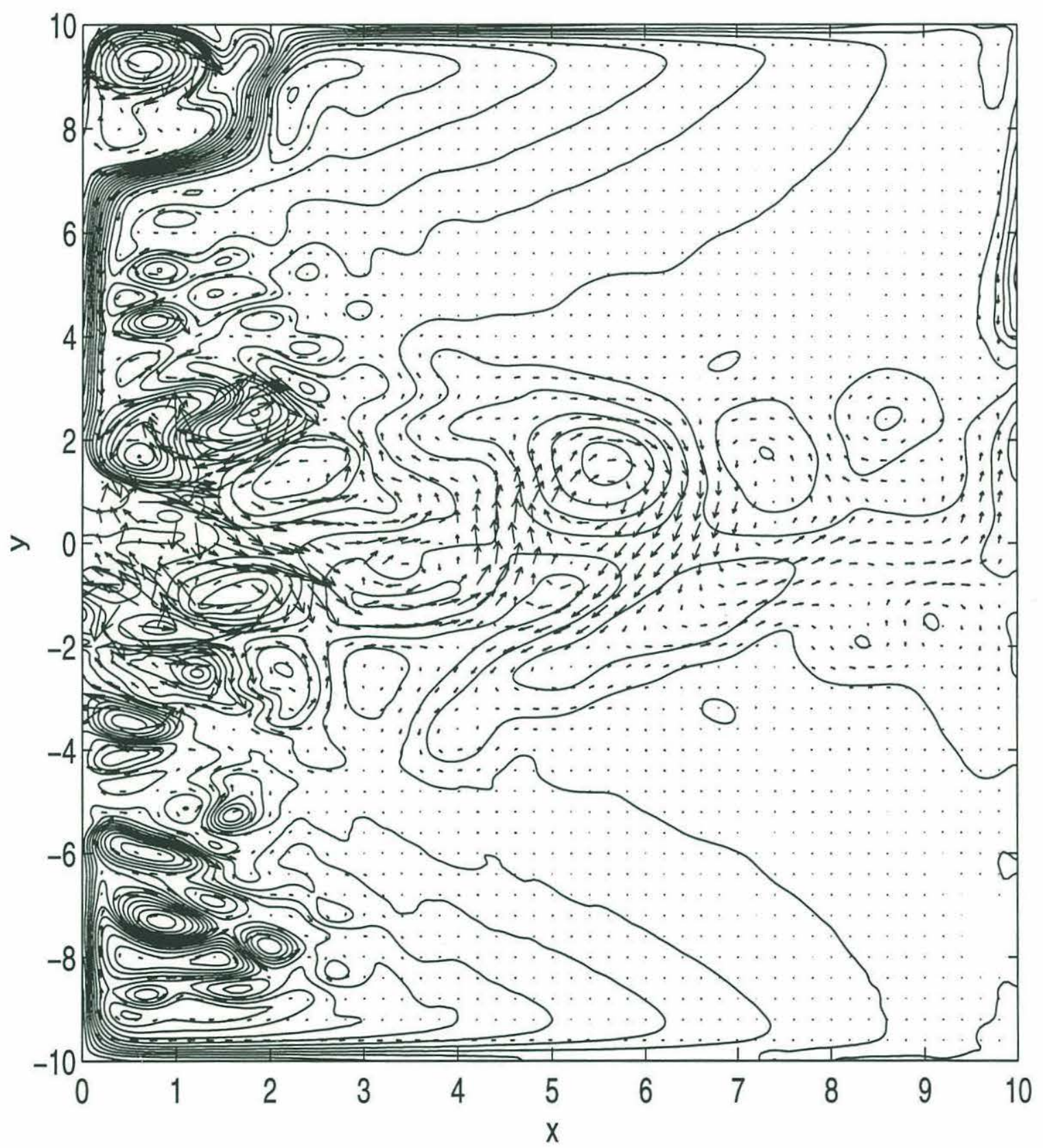

Figure 3.10: - Instantaneous fields for very nonlinear run $(R e=500)$ at time $t=2900$. Cyclonic and anti-cyclonic eddies of various scales populate the basin. 
deformation radius away.

As with the run at intermediate Reynolds number, the time-averaged fields provide some order to this complicated tangle. Shown in Figure 3.12, the time-averaged height field reveals a mean cross equatorial flow, just as in the low Reynolds number runs, with a very intense western boundary current. However, distinguishing this run is the large feature that resides just north of the equator. This large cyclonic eddy enhances the transport of the western boundary current and redirects a portion of the fluid out into the interior and back to the northern hemisphere where it migrates its way into the interior. The magnitude of this feature is directly linked to the substantial inertial overshoot of fluid in the western boundary current.

To further understand the behavior of the mean field, it is helpful to examine the meridional mass transport within the basin, integrated from the western boundary:

$$
T_{M}=\int_{0}^{x} h v d x
$$

This quantity would be equivalent to the mass transport stream function were the system horizontally non-divergent. Since the experiment includes weak upwelling throughout the domain, it is necessary to consider this quantity the meridional transport only. In regions of intense western boundary flow, streamlines and meridional transport contours must be nearly coincident, but not in regions of the interior.

In Figures 3.13 and 3.14 the function $T_{M}$ is contoured for both low and high Reynolds number flows. The volume of fluid passing through the basin is $0.1 H c L_{D}$ in both cases, and half of the volume is upwelled in either hemisphere. Observe that in the more frictional case, Figure 3.13 , the -0.075 contour extends quite far south, nearly to the equator. Almost three quarters of the fluid entering the basin follows the western boundary to the equator. The contrasting, more nonlinear case shown in Figure 3.14 reveals that fully all of the water that eventually will upwell in the northern hemisphere of the model extends down to the equator.

It is important to distinguish this eddy-like feature of the mean field from a stationary, recirculating eddy. The appearance of closed contours in the mean suggests that 


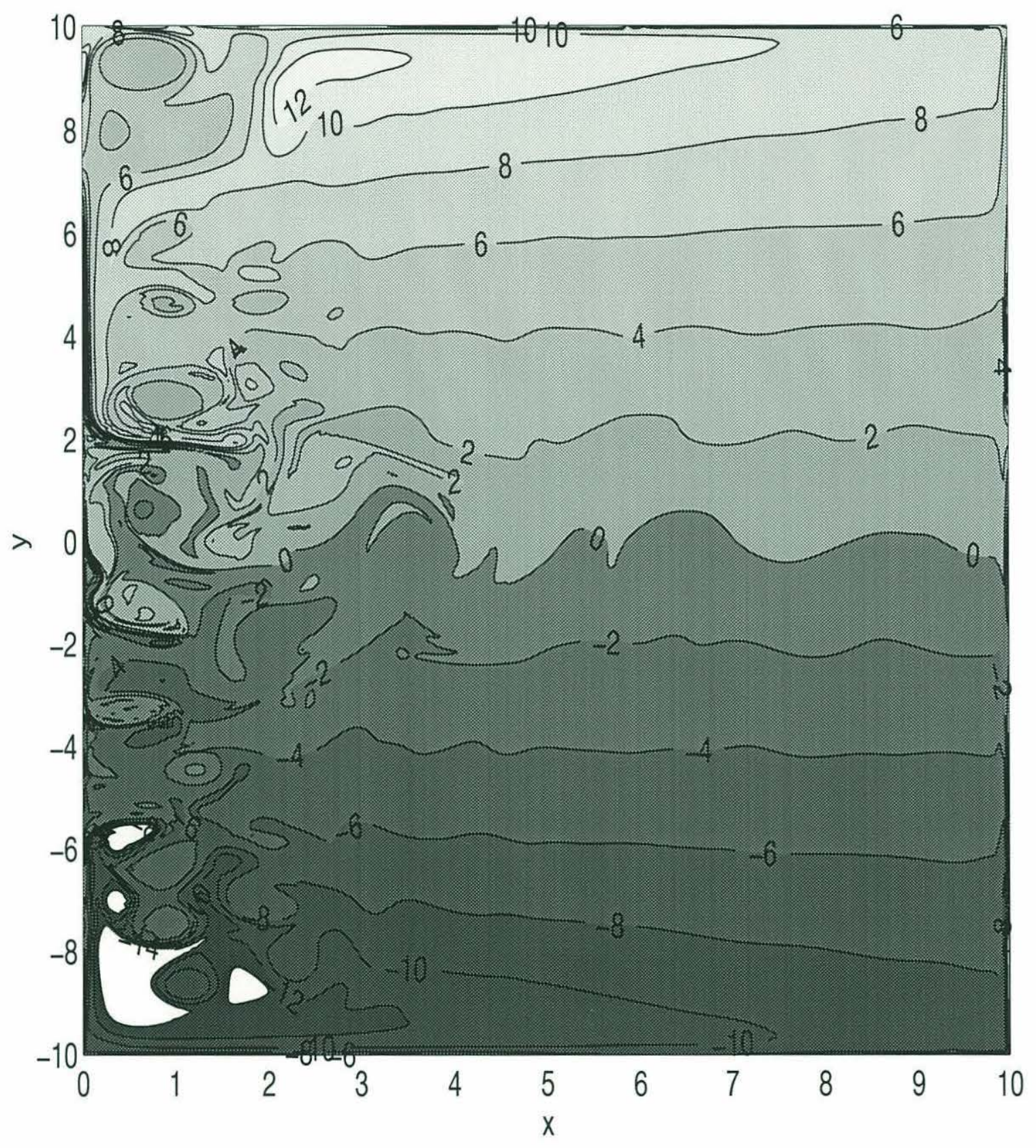

Figure 3.11: Instantaneous PV field for very nonlinear run $(R e=500)$ at time $t=2900$. Positive potential vorticity has the lighter shades and negative darker. Pure white corresponds to negative values less than 12 . 


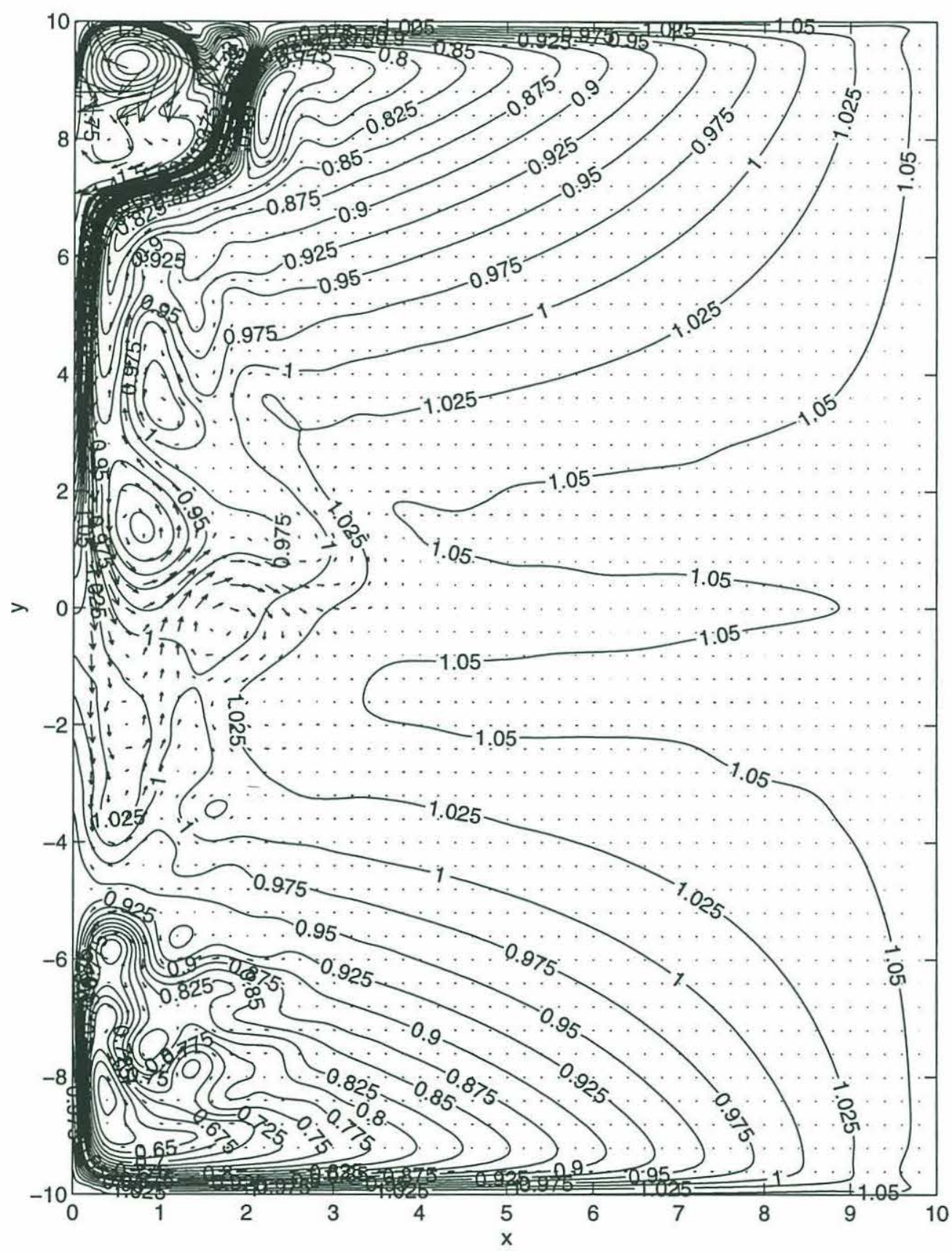

Figure 3.12: Time-averaged fields for the $R e=500$ run shown in Figure 3.10. 


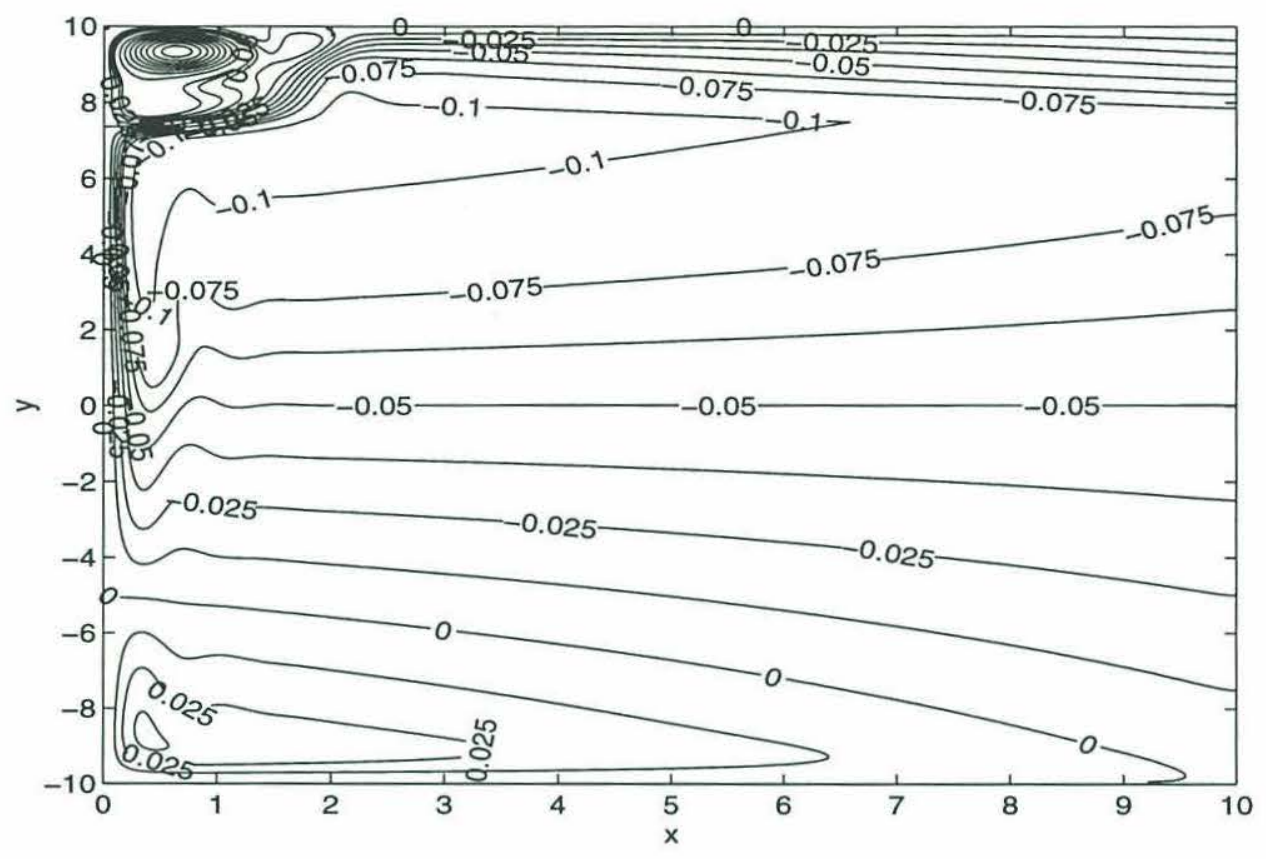

Figure 3.13: Time-averaged meridional transport for the $R e=50$ run. The volume flux through the source and sink is $0.1 H c L_{D}$.

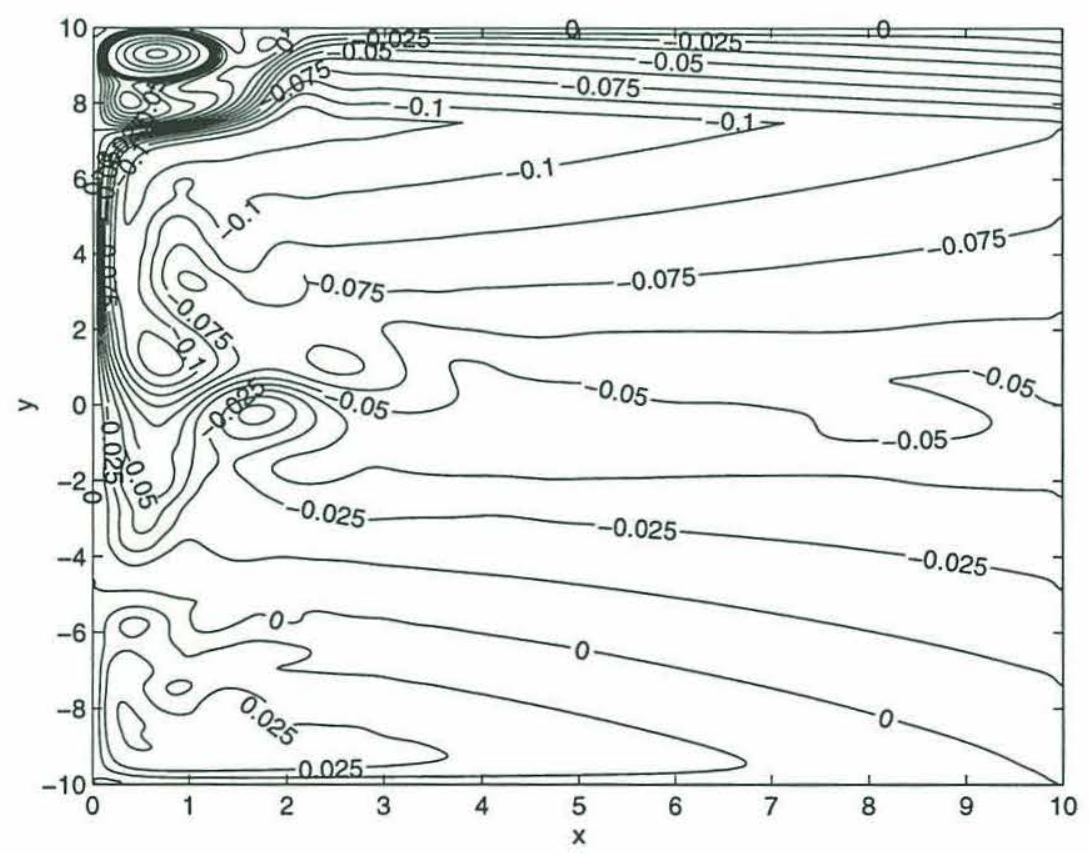

Figure 3.14: Time-averaged meridional transport for the $R e=500$ run. $S_{0}=0.1 H c L_{D}$. 
water is trapped in this region just north of the equator. To the contrary, the coherent structures in the instantaneous snapshots grow, migrate southward, and decay continually. I believe the appearance of the mean eddy is an indication that the location simply experiences protracted eddy growth or sustenance before it ejects anomalies southward across the equator.

One indication that there is no trapped fluid within this recirculation is found in the time-averaged potential vorticity field shown in Figure 3.15. There is no indication of isolated patches of anomalous potential vorticity in the region of the mean eddy, and it is unlikely that potential vorticity would be shifting continually back and forth between portions of the recirculation. Thus the mean-eddy does not reflect a local stationary recirculation in the sense of trapped fluid.

Also visible in Figure 3.15 is the strong gradient of potential vorticity at the western boundary. Indeed, contours in the mean remain considerably close to $f$-contours, except in the immediate vicinity of the western boundary where height variation and particularly velocity shear are extreme. With no slip boundary conditions, the zero potential vorticity contour extends several deformation radii up the western boundary. As a reality check, it is helpful to reconsider the observations of mean velocity in the Atlantic ocean shown in Figure 1.3. Estimating the relative vorticity in the strong shear zone suggests that

$$
\begin{aligned}
\frac{V}{\delta_{x}} & =\frac{.5 m s^{-1}}{50 k m} \\
& =10^{-5} s^{-1}
\end{aligned}
$$

Values of the planetary vorticity scale with

$$
\begin{aligned}
\beta L_{D} & \cong 2 \times 10^{-11} \mathrm{~m}^{-1} \mathrm{~s}^{-1} \cdot 250 \mathrm{~km} \\
& \cong 5 \times 10^{-6} \mathrm{~s}^{-1}
\end{aligned}
$$

Thus the prospect of negative values of mean planetary vorticity creeping up the western boundary is not inconsistent with data. 


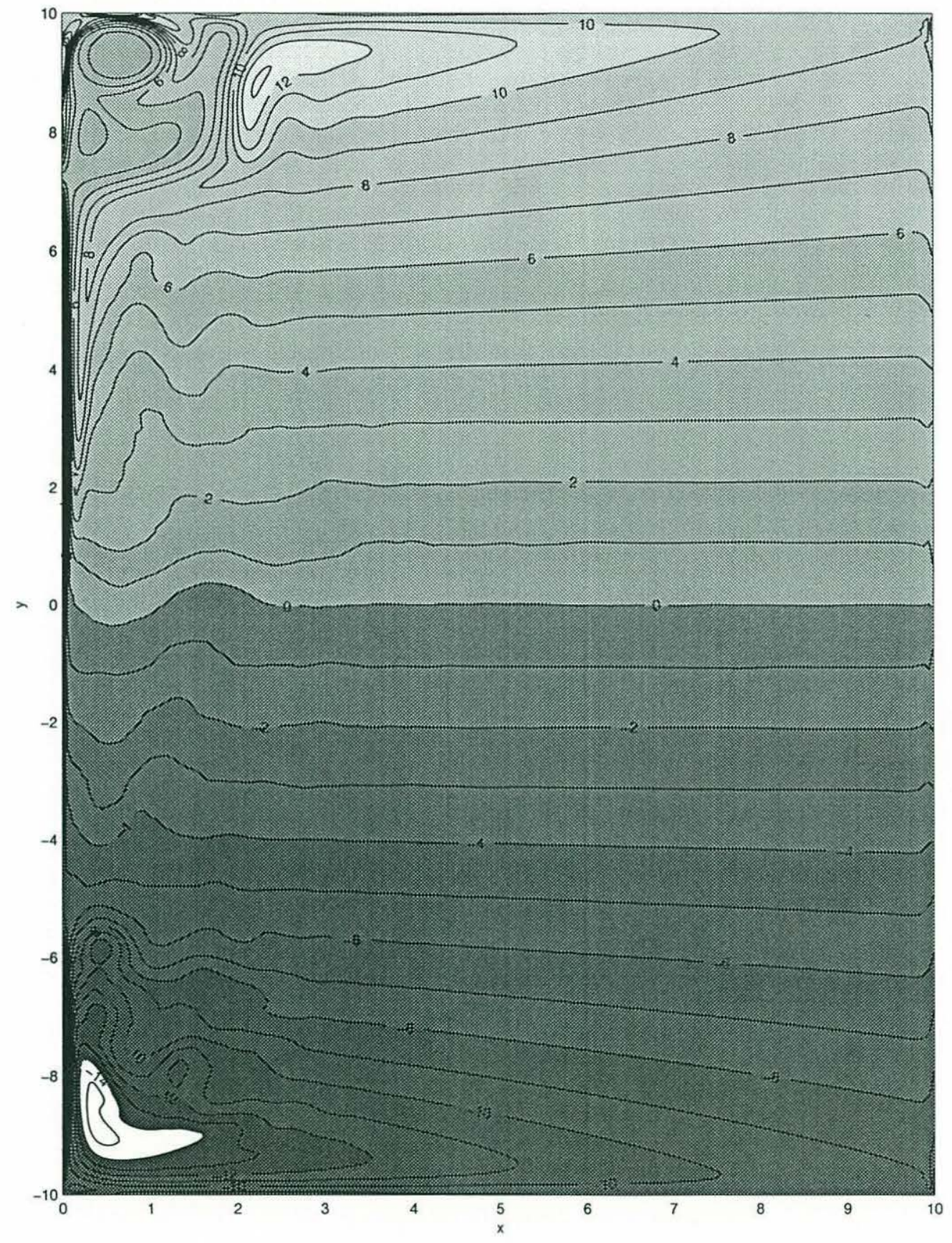

Figure 3.15: Time-mean potential vorticity for Run U8 $(R e=500)$. 


\subsection{Summary}

The numerical model successfully transports fluid across the equator for a range of Reynolds numbers. At low Re, cross-equatorial flow is laminar, and the flow is well predicted by linear theory. At a critical Reynolds number, the boundary layer becomes unstable, and the model creates cyclonic eddies which propagate periodically across the equator. Very high Reynolds number flow is characterized by considerably more variability, with cyclonic and anti-cyclonic features covering an extensive portion of the tropical ocean, particularly near the western boundary and equator. Regardless of strength of the nonlinearity, the system adjusts so as to allow cross-equatorial flow. The subject of the next chapter is the characterization of the potential vorticity transformation in this flow. 


\section{Chapter 4}

\section{Vorticity Analysis and Sensitivity}

This chapter returns to the dynamical problem of vorticity transformation in inertiofrictional cross-equatorial flow and quantitatively analyzes the vorticity fluxes in the set of numerical experiments described in Chapter 3. The first section defines the $\mathbf{J}$ vector as the primary tool of the analysis and examines the fluxes for flows of different Reynolds number. The dynamical balances of the time-mean western boundary layer is subsequently decomposed.

At the end of the chapter are sections that test the sensitivity of the vorticity fluxes to different model configurations. In particular, the effect of free-slip boundary conditions and non-uniform upwelling distributions are considered.

\subsection{Time-averaged Vorticity Analysis}

\subsubsection{J-vectors}

\section{Definitions}

By averaging Equation 2.16 over time-scales that are long compared to the time-scales for fluctuations in the system, one obtains the steady-state vorticity balance. Letting overbars with a $t$ superscript denote time averaging, the steady-state vorticity equation 
becomes

$$
\overline{\mathbf{u} \cdot \nabla \zeta}^{t}+\bar{v}^{t}+\overline{(y+\zeta) \nabla \cdot \mathbf{u}}^{t}={\overline{A_{H} \nabla^{2}(\zeta)}}^{t},
$$

expressing the balance of of four components, the nonlinear advection of relative vorticity, advection of planetary vorticity, stretching (which includes a nonlinear component), and friction.

Equation 4.1 can be further simplified by expressing it in flux form. In terms of the absolute vorticity, $\zeta_{A}=y+\zeta$, Equation 4.1 is restated, dropping the superscripts,

$$
\overline{\nabla \cdot\left(\mathbf{u} \zeta_{A}\right)}=\overline{A_{H} \nabla^{2}(\zeta)} .
$$

Rearranging terms,

$$
\nabla \cdot \mathbf{J}=0
$$

where

$$
\begin{aligned}
\mathbf{J} & =\overline{\mathbf{u} \zeta_{A}}-A_{H} \nabla(\bar{\zeta}) \\
& =\overline{\mathbf{u}} \overline{\zeta_{A}}+\overline{\mathbf{u}^{\prime} \zeta_{A}^{\prime}}-A_{H} \nabla(\bar{\zeta})
\end{aligned}
$$

Here, the primed variables represent deviations from their time-mean quantities. Physically, J-vectors represent horizontal vorticity fluxes within the fluid layer. There is zero cross-isopycnal flux, and the field is horizontally non-divergent. Although the significance of the vorticity equation has an extensive history dating back to the last century, the $\mathbf{J}$-vector formulation for three dimensional systems is more recent (Haynes and McIntyre (1987)). In that work, J-vectors depict the vorticity fluxes along isentropic sheets, whose relationship to a single layer model follows directly from an isentropic coordinate representation. Indeed the shallow-water model can be considered to represent a single isentropic layer of the ocean.

It is convenient to divide the vorticity flux into three components, one due to mean advection of mean absolute vorticity, another to the eddy flux of absolute vorticity, and the third resulting from the flux of vorticity due to frictional forces acting in the system:

$$
\mathbf{J}=\mathbf{J}_{m n}+\mathbf{J}_{\text {ed }}+\mathbf{J}_{\text {fric }} .
$$


The simplicity of the $\mathbf{J}$-vector notation is now evident. By considering the massweighted potential vorticity rather than the full potential vorticity, the forcing in the height equation is incorporated implicitly in the analysis. As a result, only three terms are responsible for a dynamical balance, as opposed to five in the full potential vorticity equation when similarly decomposed. Of course, this formulation has its cost: the loss of any dynamical information associated with the forcing in the height equation.

Since $\mathbf{J}$ is non-divergent, the flux entering any region must balance with flux leaving. Integrating Equation 4.3 over any area, $A$, bounded by a border, $\Gamma$, yields

$$
\oint_{\Gamma} \mathbf{J} \cdot \hat{n}=0 .
$$

The numerical approximation to the $\mathbf{J}$-vectors specific to the discretization is described in Appendix A.4.

\subsubsection{Analysis and Numerical Results}

\section{Linear Analysis}

Before proceeding to the numerical $\mathbf{J}$-vector plots, it is instructive to consider results for linear flow first. When the dynamics are linear, the $\mathbf{J}$-vectors reduce to the following,

$$
\begin{aligned}
\mathbf{J}_{e d} & =0 \\
\mathbf{J}_{m n} & =y \mathbf{u} \\
\mathbf{J}_{f r i c} & =-A_{H}\left(\frac{\partial^{2} v}{\partial x^{2}}, \frac{\partial^{2} v}{\partial x \partial y}\right) .
\end{aligned}
$$

Embedded in the above approximations, are the assumptions that the flow is steady and that relative vorticity is small compared to planetary vorticity and the boundary layer approximation $\frac{\partial}{\partial x} \gg \frac{\partial}{\partial y}$.

It is important to note that the advective meridional vorticity flux at any location is only a function of the meridional velocity and the latitude: $\mathbf{J}^{(y)}=v y$. As a result, 
the meridional flux of $\mathbf{J}$, while non-zero at all mid-latitudes with non-zero $v$, must vanish at $y=0$. At the equator in the linear model, there can be no advective flux of vorticity at any longitude, regardless of the meridional velocity. The meridional flux due to friction,

$$
J_{\text {fric }}^{(y)}=-A_{H} \zeta_{y},
$$

is not necessarily zero at the equator. However, integrating this quantity from the western boundary gives

$$
-A_{H} \int_{0}^{x} v_{x y} d x=-A_{H}\left(v_{y}(x)-v_{y}(0)\right) .
$$

The rightmost term on the right-hand side is zero with no slip boundary conditions, and the remaining contribution is small if the integration is carried into the interior. If the boundary layer structure is independent of $y$ and the interior fields are symmetric about the equator, then the meridional flux of vorticity due to friction is exactly zero. Relaxing these constraints, but integrating across the domain, $x=x_{E}$, shows that the net integrated frictional flux of vorticity at any latitude is in fact zero. The remaining discussion ignores any contribution to the meridional flux due to friction.

Recall the Stommel and Arons flow with simple Munk boundary layer dynamics described in section 3.3. The meridional flow in the interior vanishes at the equator, but the boundary layer flow remains. It carries the necessary mass across the equator to upwell in the southern hemisphere.

Equation 4.6 requires that the net integrated flux of vorticity into a sub-domain of the basin that is bounded by the equator, the western and eastern boundaries and a line of latitude, $y_{0}$, north of the equator, must be zero. The vorticity flux across each portion of the boundary can be considered independently. From the north,

$$
\begin{aligned}
I_{\text {North }} & =\int_{0}^{x_{E}} y_{0} v d x \\
& =y V_{\text {North }},
\end{aligned}
$$

where $V_{N o r t h}$ is the total mass transport per unit depth through the northern boundary 
of the sub-domain. Since the net transport across this latitude is southward, there is a net vorticity flux into the region across this boundary.

Since the equator is a boundary of vorticity flux, there is no contribution to subdomain through the southern boundary. In addition there is no flow through the eastern boundary and no eastern boundary current which might result in a frictional flux there. As a result, the vorticity flux through the eastern boundary is zero also. The only sink for $I_{N o r t h}$ is the western boundary, and it results from the strong shear in the boundary current:

$$
I_{W e s t}=-A_{H} \int_{0}^{y} \frac{\partial^{2} v}{\partial x^{2}} d y .
$$

Thus, vorticity enters the region through the northern boundary and leaves through the western boundary, and this pathway resides entirely in the northern hemisphere.

It is possible to further dissect the structure of the vorticity fluxes by considering the structure of the velocity field. Although the net transport at middle latitudes is negative, in the interior there exists a slow poleward velocity, and therefore an accompanying weak poleward vorticity flux (i.e., out of the domain). This poleward flux is compensated, and sometimes overcompensated, by the equatorward flux in the intense western boundary current. The domain bounded by the equator, the meridional boundaries and the line of latitude, $y$, can be dissected by a line, $x_{0}(y)$, such that

$$
\int_{x_{0}}^{x_{E}} v d x=0 .
$$

All $\mathbf{J}$ entering the region east of $x_{0}$ leaves again through the northern boundary of the subdomain. All $\mathbf{J}$ entering west of $x_{0}$ is expelled through the western boundary.

A schematic diagram of the vorticity fluxes under linear dynamics is shown in Figure 4.1. The diagram is somewhat exaggerated in the zonal scale in order to broaden the western boundary current with respect to the basin dimension. The strong southward flux at any latitude, $y=y_{0}$, is marked near the western boundary, as is the bounding vorticity streamline, $x=x_{0}(y)$. The vorticity flux west of $x_{0}$ must exit the basin at the western boundary whereas the vorticity flux immediately to its 
east is exported by the northward Sverdrup flow of the interior.

For completeness, it is useful to understand $\mathbf{J}$-vectors south of the equator. The same arguments above apply to a sub-domain that is bounded to the north by the equator and to the south by a line of latitude. With $y<0$, the vorticity fluxes due to the mean field are reversed, though the frictional flux is not. Thus south of the equator, the $\mathbf{J}$ vector diagram is inverted from its northern-hemisphere counterpart.

The $\mathbf{J}$-vector quiver plot for a numerical experiment with $R e=5$ (U1) is presented for comparison in Figure 4.2. Only a western subdomain, with $x=[0,2]$ and $y=$ $[-5,5]$, of the basin is illustrated to emphasize the fluxes associated with the western boundary current. An equatorword vorticity flux is visible near the western boundary in both hemispheres veering westward and out the western boundary by frictional processes. In addition, a portion of the vectors in the eastern part of the boundary current turn eastward toward the interior where a weak eastward and northward flux of vorticity is associated with the interior flow. These vectors are difficult to see in the diagram due to their small magnitude.

An alternative presentation reveals more clearly the vorticity fluxes in the basin. The horizontal non-divergence of the vector field permits the construction of a potential function, $\Psi$, similar to the streamfunction for a two-dimensional velocity field such that

$$
\mathbf{J}=\mathbf{k} \times \nabla \Psi .
$$

Contours of this field are streamlines of the vorticity flux; lines of constant $\Psi$ are tangent to the $\mathbf{J}$-vectors. An example for the linear run U1 is presented in Figure 4.3. Although the vorticity flux itself on any given line is not constant, contours of equal interval in $\Psi$ demarcate tubes of constant vorticity flux. Regions of large gradients in $\Psi$ (i.e., with compressed contours) represent regions of intense vorticity transport, and conversely, weak gradients correspond to weak flux of $\mathbf{J}$.

In Figure 4.3, the strong equatorward flux of vorticity is visible near the western boundary. The trend of some vorticity streamlines turning toward the western bound- 


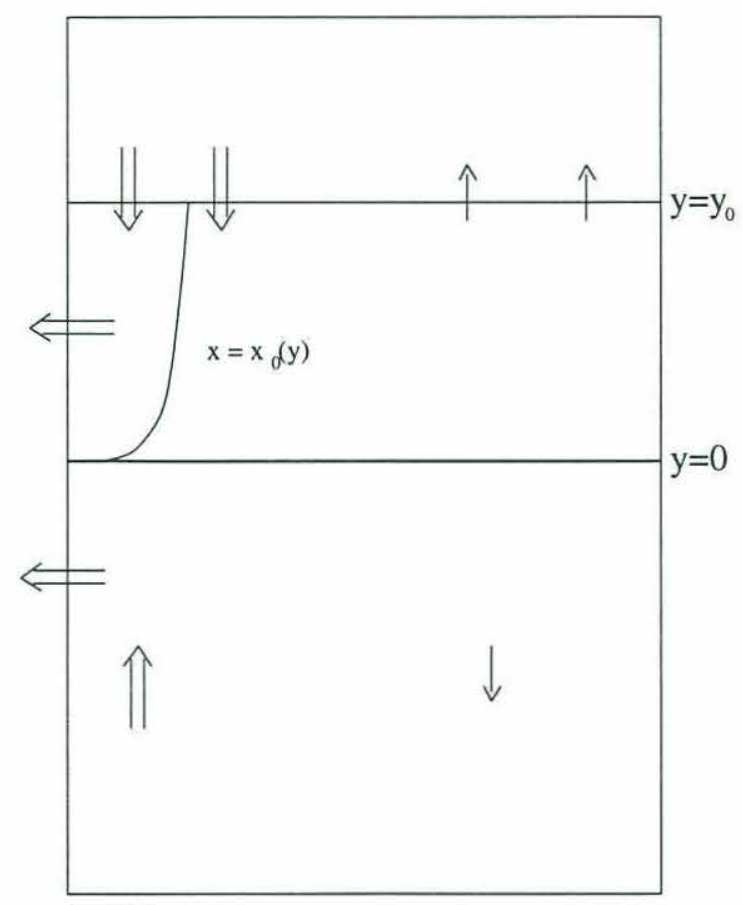

Figure 4.1: Schematic of the J-vector fluxes for a linear, Stommel and Arons solution with a Munk boundary layer.

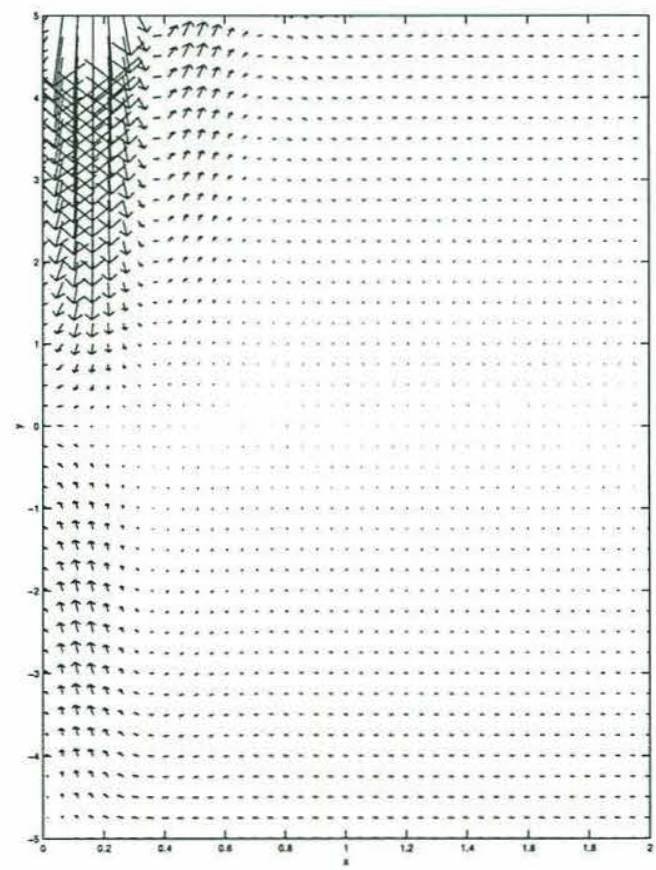

Figure 4.2: $\quad \mathrm{J}$-vectors for run $\mathrm{U} 1, R e=5$. The aspect ratio emphasizes north-south fluxes. 


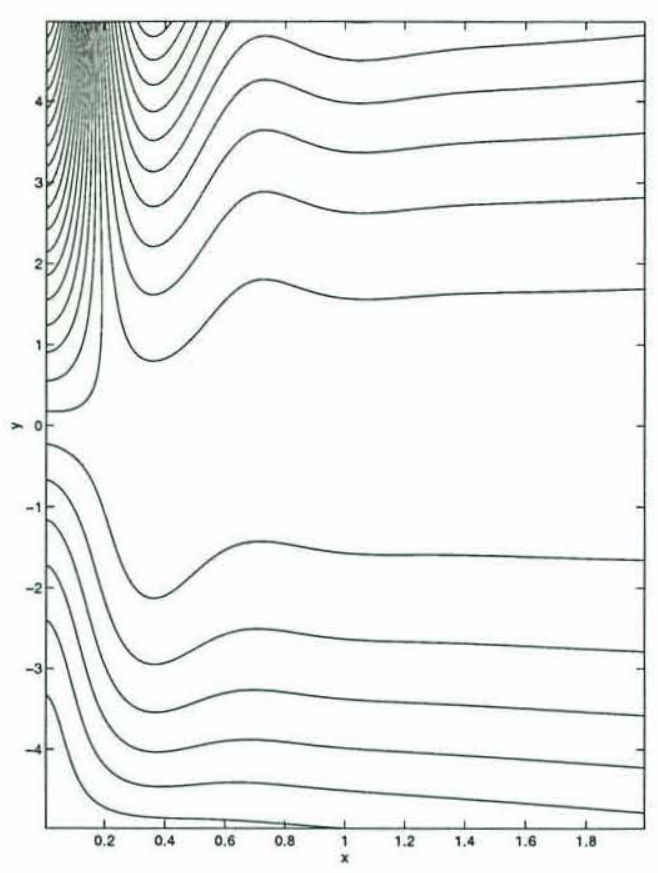

Figure 4.3: Streamfunction of $\mathrm{J}$ for run $\mathrm{U} 1, R e=5$.

ary indicates the transfer of vorticity to that boundary, and the weak poleward and eastward flux in the interior is now considerably more visible. The demarcation line, $x_{0}$ separating $\mathbf{J}$ having these two destinies is not drawn in the figure, but its existence and location is easily imagined.

\section{Weakly Nonlinear}

Moving to the next stage in complexity allows nonlinearity to enter the dynamics, but only in the meridional momentum equation, though I still restrict the flow to be steady. In this scenario and neglecting the meridional flux due to friction,

$$
\begin{aligned}
\mathbf{J}_{e d} & =0 \\
\mathbf{J}_{m n} & =\left(y+\frac{\partial v}{\partial x}\right) \mathbf{u} \\
\mathbf{J}_{\text {fric }} & =-A_{H}\left(\frac{\partial^{2} v}{\partial x^{2}}, 0\right) .
\end{aligned}
$$


Now the total advective meridional vorticity flux across a line of latitude is

$$
\begin{aligned}
I_{\text {North }} & =\int_{0}^{x_{E}}\left(y+\frac{\partial v}{\partial x}\right) v d x \\
& =y V_{\text {North }}+\int_{0}^{x_{E}} \frac{1}{2} \frac{\partial v^{2}}{\partial x} d x . \\
& =y V_{\text {North }}+\frac{1}{2}\left(v^{2}\left(x_{E}\right)-v^{2}(0)\right)
\end{aligned}
$$

If the boundary conditions are no-slip, this reduces to the same vorticity flux as in the linear case. The net meridional vorticity flux is again given by the integrated velocity advecting planetary vorticity across the line of latitude.

As in the purely linear case, the equator is a boundary across which no vorticity can be advected. However, one feature distinguishes this weakly nonlinear case from the purely linear one. When the dynamics are linear, there is zero meridional vorticity flux at all longitudes on the equator. With the addition of weak nonlinearity, Equation 4.14b, there can be cross-equatorial flux of $\mathbf{J}$ at all longitudes, but the net flux, when integrated across the domain, must be zero.

Consider the net cross-equatorial flux of vorticity between any two longitudes, $x_{1}$ and $x_{2}$ :

$$
I_{\text {Equator }}=v^{2}\left(x_{2}, 0\right)-v^{2}\left(x_{1}, 0\right) .
$$

Between any longitudes of identical meridional velocity, there is no net flux. Thus when the eastern and western boundaries are used, there is no integrated flux, and similar sub-domains lie between all locations of flow reversal.

The streamfunction of $\mathbf{J}$ for a weakly nonlinear, but steady numerical experiment, run U2 is shown in Figure 4.4. In this example, the streamlines do cross the equator near the intense center of the western boundary current, but return both to the west and to the east northward such that the integrated flux is zero.

\section{Fully Nonlinear}

Adding further complexity by allowing nonlinearity to enter the dynamics in the zonal momentum equation and through time dependence renders the problem analytically 


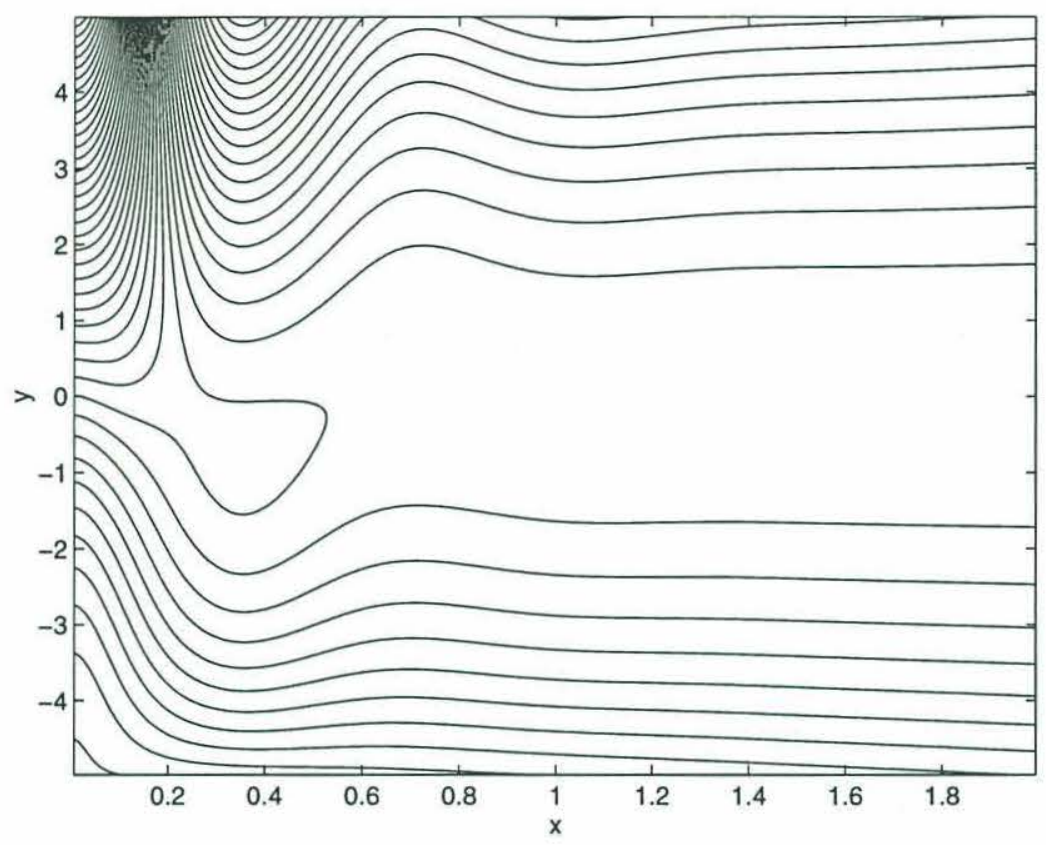

Figure 4.4: Streamfunction of $\mathbf{J}$ for run $\mathrm{U} 2, R e=20$.

intractable. However, it is possible to rely on the numerical calculations in this limit. Using the full formulation for the $\mathbf{J}$-vectors as in Equation 4.4a, the stream function for the high Reynolds number run U8 is shown in Figure 4.5 for the sub-domain of the system bounded by latitudes $y= \pm 5$ and longitudes, $x=0$ and $x=2$.

The immediately striking result is that the streamfunction diagram appears remarkably similar to that in the weakly nonlinear run. Although the system has changed quite dramatically from the linear limit (compare again Figures 3.7 and 3.10), the dynamical picture given simply by the net vorticity flux streamfunction, $\Psi$, is not dramatically different.

Vorticity flux streamlines enter the sub-domain through the northern boundary. Some veer westward to the western boundary, and others diverge to the east and northward around the mean eddies that reside in these regions. Vorticity transported along these paths eventually work its way around to the weak interior flow and out of the domain across latitude $y=5$ (not shown). Some streamlines again propagate across the equator, with one extenting quite far. However, the vorticity flux associated 


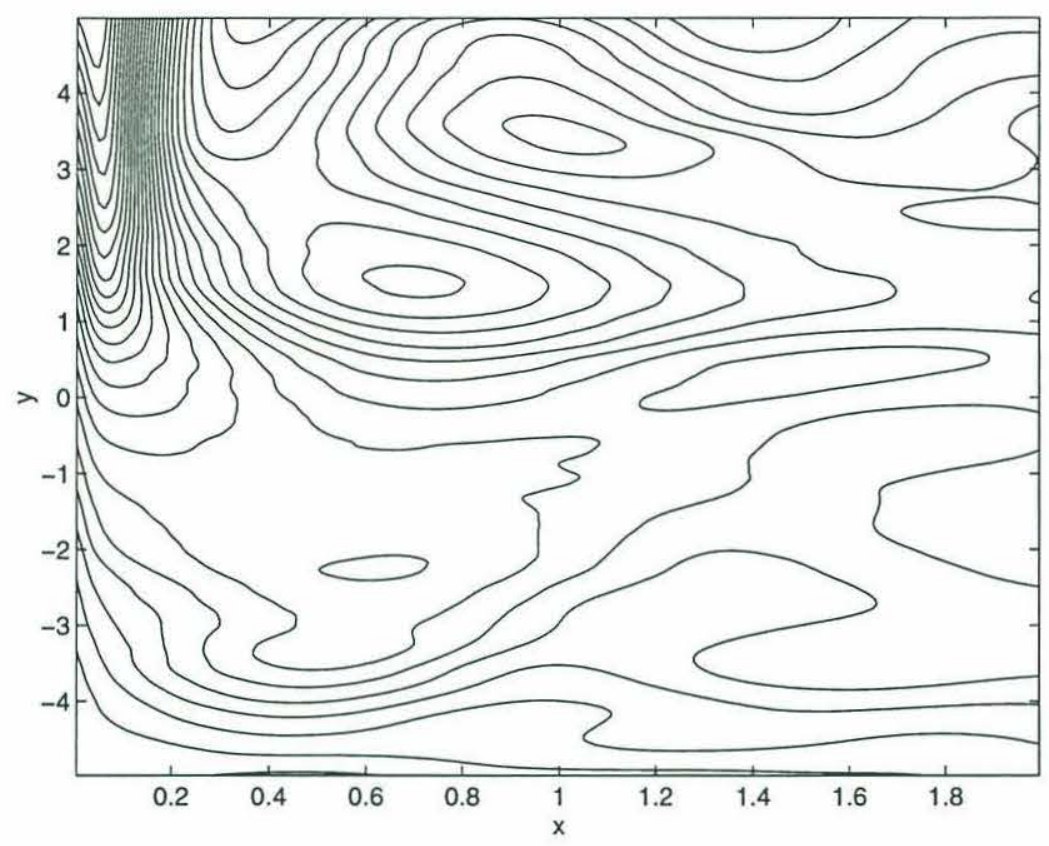

Figure 4.5: Streamfunction of J for run U8, $R e=500$.

with this one stream tube is quite small, and even this returns northward to intersect the western boundary very near to the equator. Thus even in the highly nonlinear case examined, vorticity fluxes southward across the equator, but the integrated flux is quite small. A calculation of this integrated flux gives $I_{E q u a t o r}=0.002$, less than a percent of the meridional vorticity flux found at latitude $y=5$.

Although the linear and nonlinear runs appear very similar using this diagnostic, an important distinction exists and can be illustrated by decomposing $\mathbf{J}$ into its individual components as in Equation (4.5). Figure 4.6 shows the contributions to $\mathbf{J}$ by each of the three different components for this run as vector plots. Note that the vector plots are not to the same scale, but can be used to infer locations where different processes are active. The magnitudes of the different components are given on the axes.

In Figure 4.6a, the mean advection of mean absolute vorticity shows a strong influx near the poleward boundary of the sub-domain. Figure $4.6 \mathrm{c}$ shows the flux due to friction. Over most of the domain, the mean frictional flux is zero. However, there is a region immediately adjacent to the western boundary where this flux is 

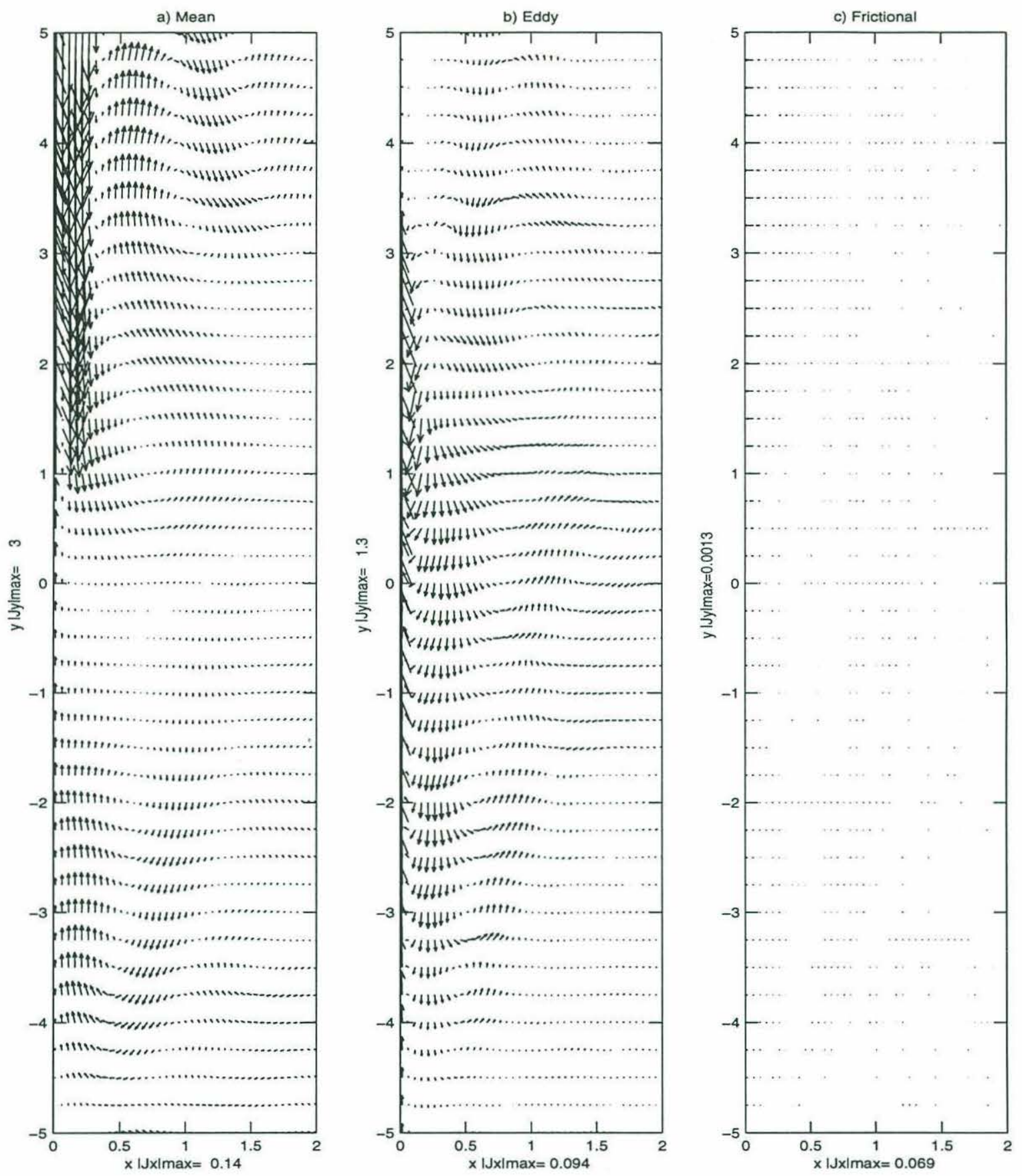

Figure 4.6: J-vector components, $J_{m n}, J_{e d}$, and $J_{f r i c}$, for run U8, $R e=500$. Note that the quivers are not to same scale, but the maximum of each vector component are given on the axes. The extreme aspect ratio causes zonal arrows to have quite small heads (particularly noticeable in the frictional plot). 
significant. It is difficult to see this in the figure due to the large aspect ratio of the plot which shrinks the heads of zonal arrows and its proximity to the western boundary. Careful examination reveals that particularly in the northern hemisphere, though also in the southern hemisphere, there is a strong westward flux of vorticity through the western boundary. These two components are qualitatively similar to those of the linear and weakly nonlinear dynamics (not shown). However, Figure 4.6b shows the contribution by the eddy field, obviously not present in the previous experiments since those integrations reached steady-state solutions.

The eddy field produces a diverging vorticity flux north of the equator. Some vorticity is transferred eastward, where it merges again with the interior flow there. But a stronger component contributes to the equatorward and westward vorticity transport, fluxing vorticity from the central portion of the boundary current southward and westward, where it can be removed by friction.

Since the quiver plots are not to the same scale and the aspect ratio of the axes is extreme, a better reflection of the relative strengths of the different components is given by the divergences of the individual components of $\mathbf{J}$. Shown in Figure 4.7 is the divergence of the three vector fields shown in Figure 4.6 in a still narrower sub-domain near to the western boundary. A positive divergence is lightly shaded and negative values are darkened. The divergence of the different components shows the intensity of $\mathrm{J}$-vector components in the vicinity of the equator and western boundary region. At the northern portion of the sub-domain, the convergence of mean $\mathbf{J}$-flux is balanced by a frictional divergence, whereas near the equator, mean and frictional fields balance a large eddy-flux convergence. Slightly further from the boundary region is a strong eddy divergence, balanced only by the effect of the mean field.

Although one effect of the eddy-field is illustrated by the $\mathrm{J}$-vector diagram of the previous section, additicnal calculations further demonstrate its role by focussing on the contribution of the eddy-field to the net westward flux. Since excess vorticity entering the sub-domain through the northern zonal boundary must be exported out 

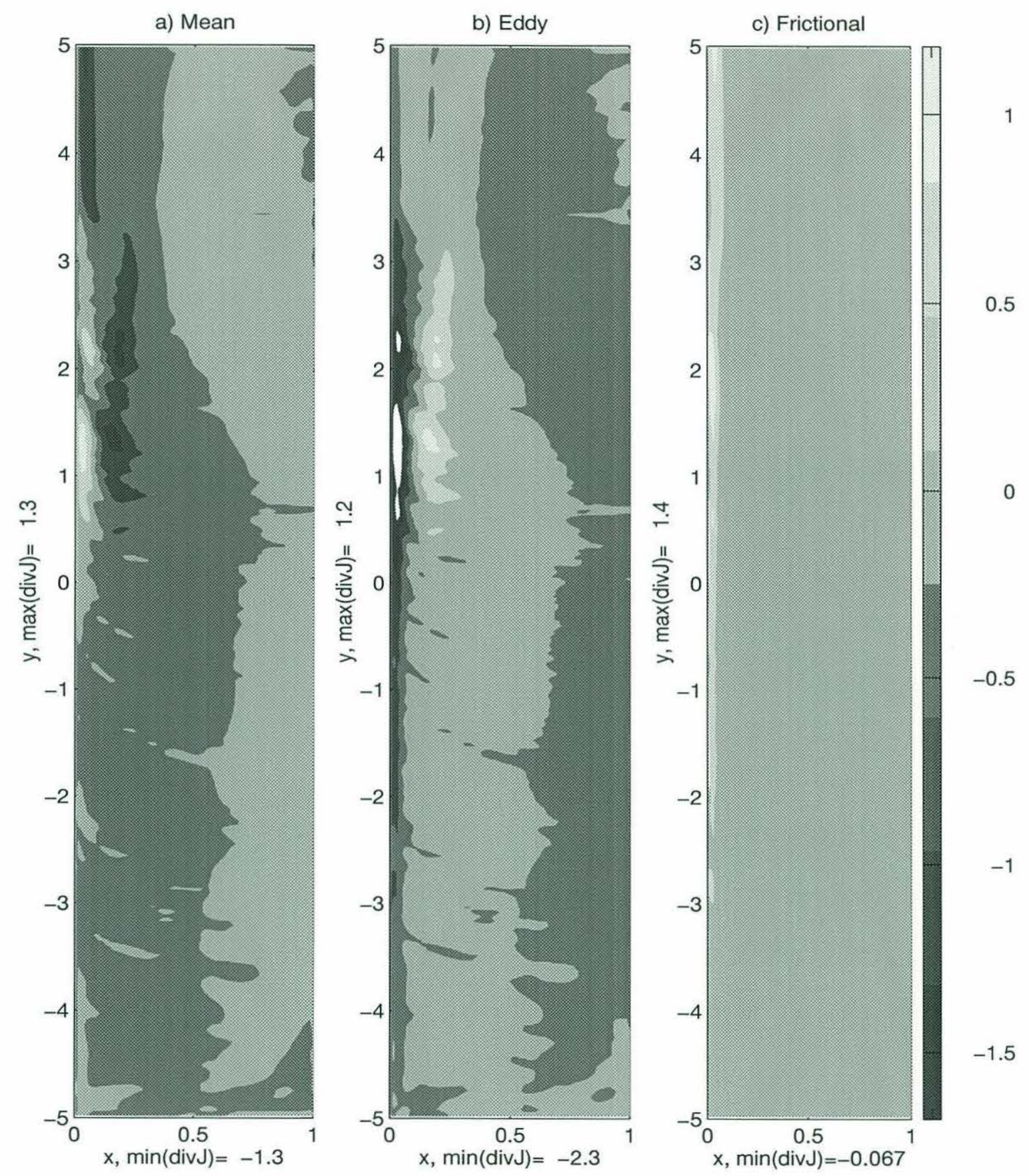

Figure 4.7: The divergences of J-vector components, $J_{m n}, J_{e d}$, and $J_{f r i c}$, for run U8, $R e=500$. Shading is identical in all three plots and maxima are given on the axes. Pure white corresponds to values less than -1.5 . 
to the west, it is interesting to inquire which processes are important at which stages of this migration.

Integral measurements are determined by decomposing Equation 4.6 into its four components:

$$
\begin{aligned}
\int_{\Gamma} \mathbf{J} \cdot \hat{n}= & \int_{y_{s}}^{y_{n}} J_{\text {west }} d y+\int_{x_{w}}^{x_{e}} J_{\text {north }} d x- \\
& \int_{y_{s}}^{y_{n}} J_{\text {east }} d y-\int_{x_{w}}^{x_{e}} J_{\text {south }} d x \\
= & I_{\text {west }}+I_{\text {north }}+I_{\text {east }}+I_{\text {south }} .
\end{aligned}
$$

where small letters donote boundaries of a variable subdomain as opposed to the full basin boundaries. Integral quantities are calculated along the boundaries $x_{w}=\epsilon$, $x_{e}=3, y_{s}=-5, y_{n}=5$, where $\epsilon$ is a small distance from the western boundary ${ }^{1}$. The different components for run U8 gives (rounded to hundredths)

$$
\begin{aligned}
I_{\text {north }} & =-0.40 \\
I_{\text {west }} & =0.43 \\
I_{\text {south }} & =-0.03 \\
I_{\text {east }} & =0.00 .
\end{aligned}
$$

Noting that these values sum to zero gives some measure of the consistency of the model in accurately carrying vorticity. As expected, the flux through the eastern and southern boundaries of the subdomain is small compared to the northern and western ones. If the subdomain is extended to the eastern boundary there is a slight increase in the frictional vorticity flux there but it is $\mathrm{O}\left(10^{-3}\right)$, consistent with expectations of no eastern boundary current.

It is also possible to decompose each geographic $I$ component into its mean, eddy and frictional elements and observe the integrated vorticity budget in a sub-domain. Table 4.1 displays the different components for this run. As expected the flux through

\footnotetext{
${ }^{1}$ As discussed in Appendix A.4, J-vectors are calculated on $h$-points of the model and are therefore staggered resting just off the boundary
} 


\begin{tabular}{|c|c|c|c|c|}
\hline & $\mathbf{J}_{\text {tot }}$ & $\mathbf{J}_{\text {mean }}$ & $\mathbf{J}_{\text {eddy }}$ & $\mathbf{J}_{\text {fric }}$ \\
\hline$I_{W}$ & .43 & 0 & .01 & .42 \\
\hline$I_{N}$ & -.40 & -.40 & 0 & 0 \\
\hline$I_{E}$ & 0 & -.01 & .01 & 0 \\
\hline$I_{S}$ & -.03 & -.03 & 0 & 0 \\
\hline
\end{tabular}

Table 4.1: Vorticity budget for the box $\left[\begin{array}{llll}0 & 3 & -5 & 5\end{array}\right]$ for run U8.

the north is almost entirely due to the mean advection of mean absolute vorticity. And the flux at the western boundary is due almost entirely to friction.

Although there appears a small eddy flux at the western boundary, this reflects the small distance, $x=\epsilon$, at which the measurement is made. At $x=0$ the entire vorticity flux must result entirely from friction since the mean and eddy flux at a solid boundary is exactly zero.

Though useful, this calculation only gives information for a single domain and is not globally illuminating. By scanning the western edge of this sub-domain from the western boundary eastward, it is possible to explore the different components as a function of longitude.

A scan of the integrated westward flux, $I_{\text {west }}(x)$ for northern and southern hemispheres of this run is shown in Figure 4.8. In solid is the total westward flux of vorticity, dashed is that due to friction. The effect of the mean field is the dotted curve and the dot-dashed is the westward flux due to the eddies. The heavy solid line shows the residual of the sum of the different integrated components around the whole sub-domain, $\Gamma$. In both figures, this residual is quite close to zero for all longitudes, consistent with Equation 4.6. In both hemsipheres, there exists a very intense westward flux due to friction at the western boundary. However, the frictional boundary layer is narrow compared to the width of the boundary current itself (compare $\delta_{M}^{\star}=0.044$ to $\delta_{I}=0.1$ ) in this high Reynolds number run. With a large portion of the full boundary layer passing outside the region where friction can act, the system adjusts by creating an eddy field, to the east of the frictional boundary layer, to transport 
vorticity from the interior of the boundary current to the frictional sublayer. This role is illustrated by the strong hump in the dot-dashed curve at $x=0.1$. Interestingly, in the northern hemisphere where the vorticity constraint in the linear and weakly nonlinear systems is most compelling, the mean field acts in fact to advect vorticity away from the boundary. The eddy and frictional components must compensate not only for the narrowing of the frictional sublayer, but also for this competing effect of the mean field. In the southern hemisphere, the mean field flux has a different sign due to the change in the sign of the Coriolis parameter, but the effect of the eddy field is still quite apparent in the narrow region immediately adjacent to the frictional sublayer.

To examine the role of the eddies in transporting vorticity meridionally, the integrated flux of vorticity between $x=0$ and $x=3$ as a function of latitude is shown in Figure 4.9. There is a large meridional vorticity flux at high latitudes due almost entirely to the mean field, but this flux decays steadily with latitude and is virtually zero at the equator. A small residual remains due to the eddy flux. This figure demonstrates that in the mean, zonally integrated sense, a system constrained to transport fluid across the equator successfully changes the sign of the vorticity of the fluid in the boundary current, even with a very small magnitude of dissipation. By the time the fluid reaches the equator, the large anomalous absolute vorticity of the boundary current is largely reduced to a small, equatorial value.

The broader effect of varying the Reynolds number can be determined by examining the ratio of the peak of the dashed curve to the maximum of the integrated frictional flux in Figure 4.8. One difficulty in defining this index is that it depends on the choice of the maximum latitude, $y_{n}$, for integration. The frictional flux extends all along the western boundary, whereas the maximum westward eddy flux is greatest within a few deformation radii of the equator (see, for example, the lightly shaded regions in Figure 4.7). Interestingly, the extent of the region of enhanced eddy flux is similar for a range of Reynolds numbers, and lies between the equator and a latitude between 
a) Northern hemisphere (mean(..), eddy(-.), fric(--), total(-), residual(heavy-))

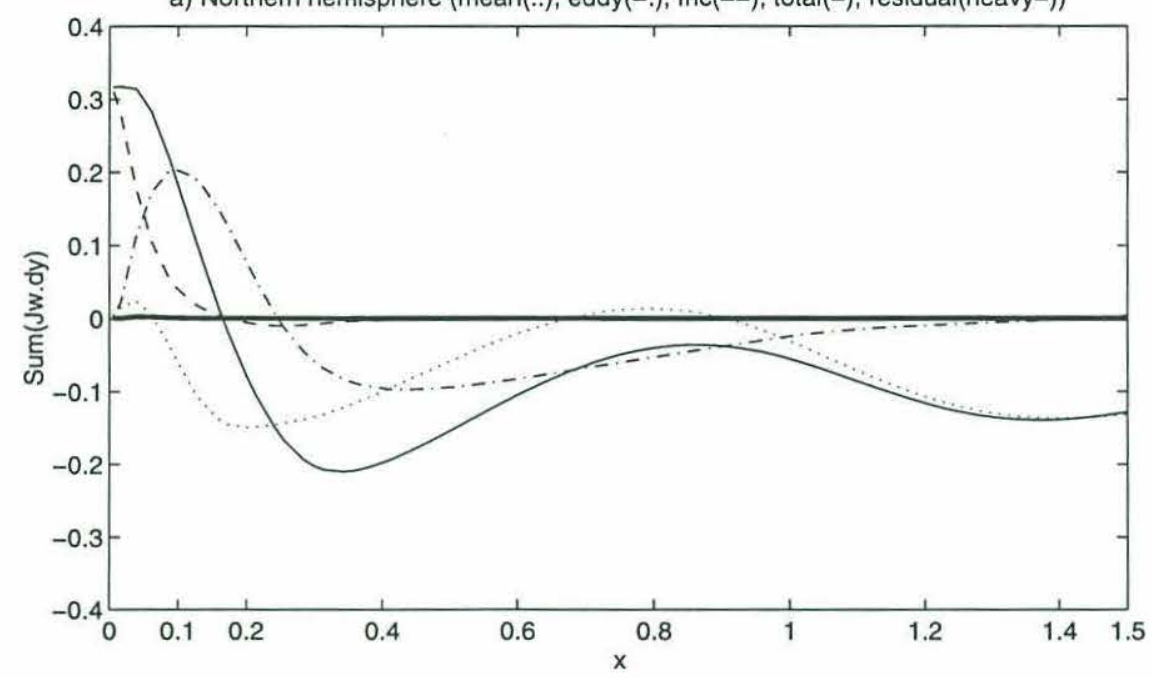

b) Southern hemisphere

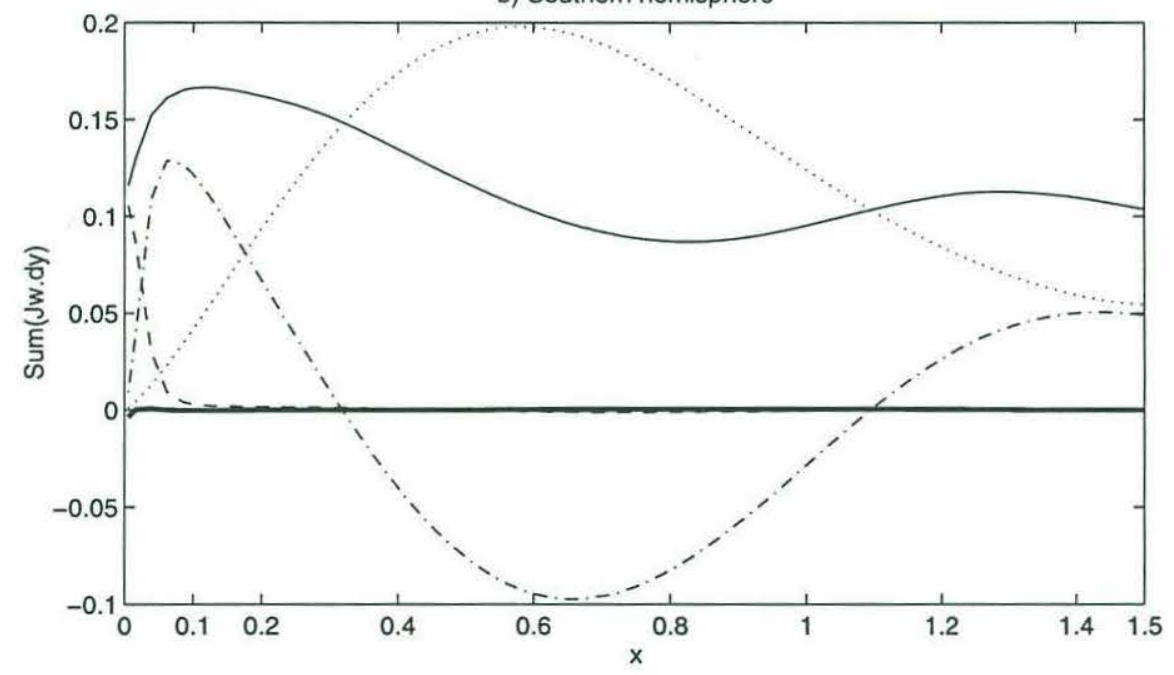

Figure 4.8: $I_{\text {west }}(x)$ for run U8, $R e=500$. The dotted line is due to the mean flux, the dashed corresponds to friction, dot-dashed to eddy, and the solid is due to the sum of all components. The heavy solid line is the sum of net fluxes around a box bounded by the longitude $x$ at the west, and the lines, $x_{e}=2, y_{n}=5$, and $y_{s}=-5$. 


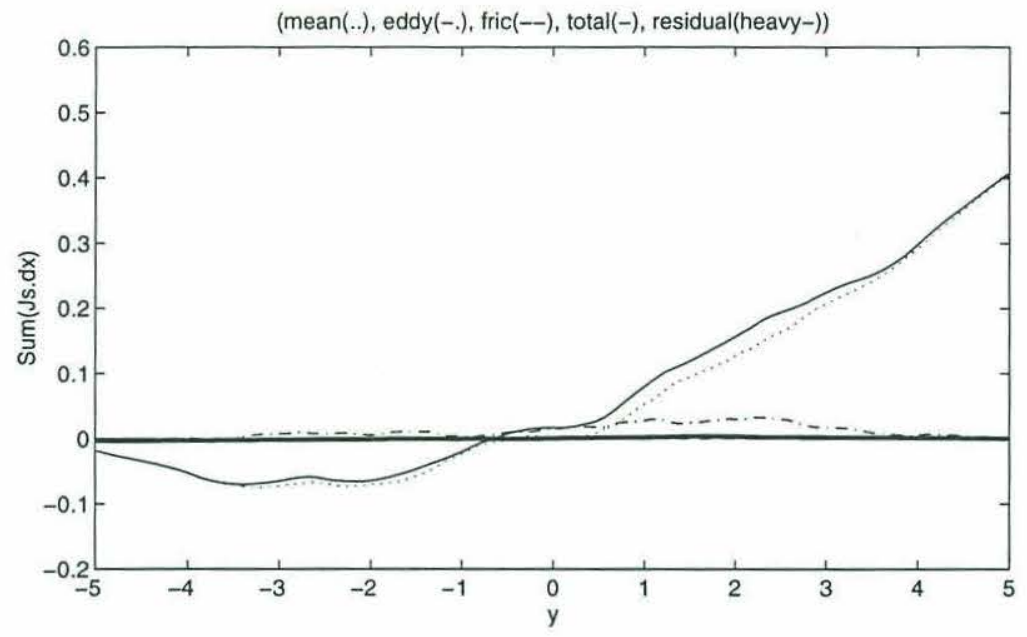

Figure 4.9: $-I_{N}(x)$ for run $\mathrm{U} 8, R e=500$. Lines are as in Figure 4.8. The large meridional flux of vorticity at latitude $y=5$ decreases to nearly zero at $y=0$. Note that the integral in this calculation extends across the basin.

$y=3$ and $y=4$ for this model configuration.

For each Reynolds number, I estimate the maximum latitude for integration as that location where the integral of the westward eddy flux has reached $98 \%$ of its value at $y=5$, which is assumed to be close to its maximum value, and define the index,

$$
E=\frac{\left[I_{w}^{e d}\right]_{\max }}{I_{w}^{\text {fric }}}
$$

to represent an integrated and normalized measure of the strength of the eddy field in transporting vorticity to the boundary. This index is calculated from numerical experiments and plotted in Figure 4.10 as a function of Reynolds numbers. At low $R e$, the integrated eddy flux is quite small. The star corresponding to $R e=32$ (run U2.A) has an index, $E=0.003$, and indicates that the critical Reynolds number for instability to occur is slightly less than this value. As the Reynolds number increases, the integrated effect of the eddies increases also, showing the growing role of the eddies in transporting vorticity westward. Included in this plot are two runs at $R e=150$ which examined variation in basin dimension. Results from both run U5.X (with $\left(L_{x}, L_{y}=(15,20)\right)$ and run U5.Y (with $\left.\left(L_{x}, L_{y}\right)=(10,30)\right)$ show remarkably good agreement with one another and in the trend of this integral calculation. 


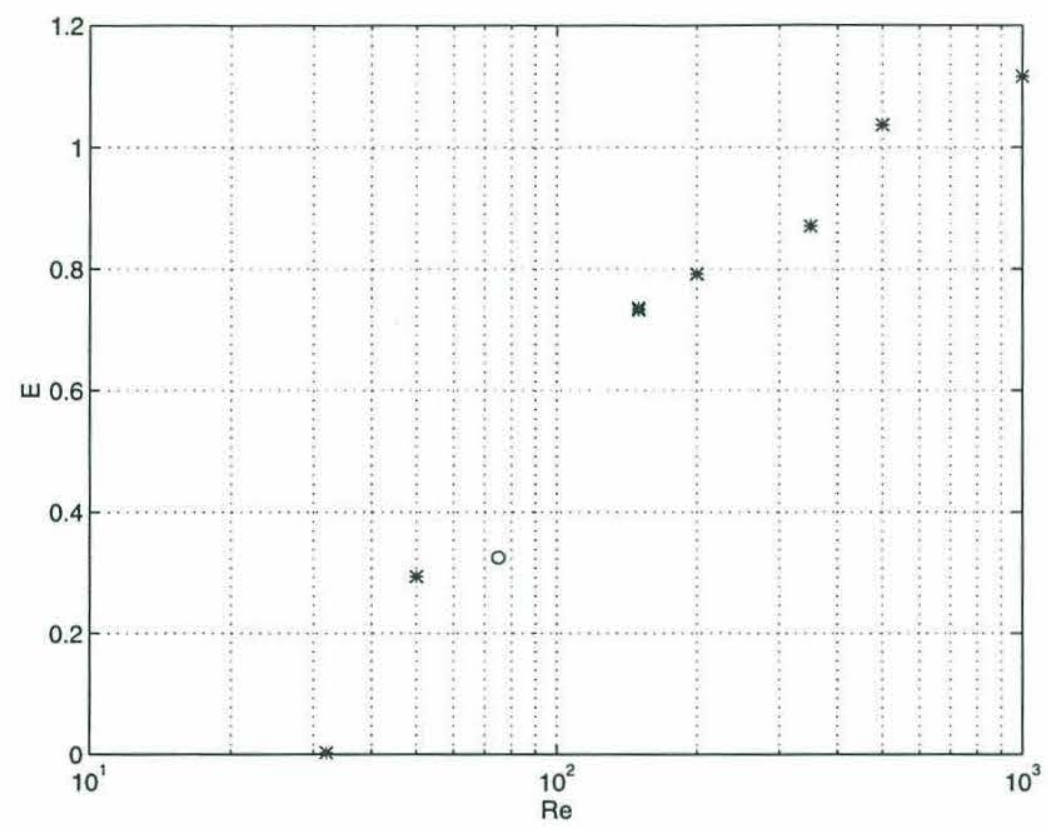

Figure 4.10: Index $E$, defined by Equation 4.14, showing the ratio of maximum integrated westward eddy flux of vorticity to the integrated dissipative flux as a function of Reynolds number. Integration is extended from the equator to the latitude where the westward eddy flux reaches $98 \%$ of its maximum value. The circle corresponds to a run with non-uniform upwelling (UFS). Runs U2.A, U4, U5.X, U5.Y, U6, U7, U8, U9, and N2 are included.

The figure suggests that the integrated eddy flux increases approximately exponentially from zero at a critical value of $R e_{c} \sim 30$ to a value of more than 1 at $R e=500$. This critical value is determined empirically, but is not drastically different from other calculations using, for example, a nonuniform upwelling distribution. The approximate exponential growth of the normalized flux is not predicted by a theory.

\subsubsection{Balances from the Time-Mean Vorticity Equation}

The vorticity balance of the linear system is obvious from the conditions of linearity. Specifically, planetary vorticity advection is balanced by vorticity diffusion. As the system becomes more nonlinear, the boundary layer structure is not obvious, but can be diagnosed from the model. This section examines the structure of the boundary layer as a function of latitude in the high Reynolds number runs. 
The time-averaged vorticity equation (4.1) is written again,

$$
\overline{\mathbf{u} \cdot \nabla \zeta}+\bar{v}+\overline{(\zeta+y) \nabla \cdot \mathbf{u}}=\overline{A_{H} \nabla^{2} \zeta}
$$

where the different terms correspond to relative vorticity advection (RVA), planetary vorticity advection (PVA), stretching (STR), and friction (FRIC). The eddy terms have not been separated from the different components of vorticity as the purpose of this section is to determine the time-mean structure of the western boundary current. The numerical diagnosis of these terms according to the discretization of the model is discussed in Appendix A.3.

For very large Reynolds number, the inertial boundary layer scale, $\delta_{I}=\sqrt{U}$, is larger than the Munk scale. In this case the system divides into multiple regions with different balances presiding. The different components of the time-averaged vorticity equation for a subdomain of run U7 near the western boundary are shaded and contoured in Figure 4.11. Frictional dissipation (Figure 4.11d) occurs entirely in the intense frictional boundary layer that resides immediately adjacent to the western boundary. Its magnitude and, to some extent, zonal scale decrease with latitude. The planetary vorticity advection ((Figure $4.11 \mathrm{~b})$ is proportional to $\bar{v}$ and therefore reveals the zonal width of the full boundary layer. The boundary current in this nonlinear run is relatively narrow in the northern portion of the sub-domain, but widens considerably near the equator. In addition, the magnitude of the planetary vorticity advection decreases substantially by this latitude. The relative vorticity advection ((Figure 4.11a) is characterized by an intense layer adjacent to the western boundary but with an oppositely signed, weaker contribution to its east. The effect of stretching is found in Figure 4.11c and is surprisingly small in all regions of this sub-domain. Of course, the contribution of stretching to the vorticity balance is significant in the interior (roughly 2 to 3 deformation radii from $x=0$ ) and away from the equator, where stretching balances planetary vorticity advection.

To better understand the balances from this plan view, the zonal profiles of the different components at 4 latitudes are shown in Figure 4.12. The upper panel shows 
the different components at $y=5$ and reveals a split of the boundary current into two dynamical regimes. The inner layer shows a three-way balance of friction (dotted), relative vorticity advection (solid), and planetary vorticity advection (dashed). There is also an outer layer that is primarily an inertial balance of RVA and PVA. As the latitude decreases, the inner layer narrows considerably, the outer layer widens, and the effect of planetary vorticity advection in the inner layer decreases. This balance remains valid far south of the equator as well.

From the input parameters it is possible to estimate the expected scales of the boundary layers. For run U7, the Munk scale is $\delta_{M}=0.0667$, the inertial scale, assuming a Stommel and Arons interior flow as in Equation 2.19, is 0.1. However, the three-way balance of Figure $4.12 \mathrm{a}$ suggests a scaling of the system such that $\delta_{I}=\delta_{M}$. Thus in this northern portion of the subdomain, there exists a slightly narrower inertial current than expected from Equation 2.19. Moving further south in the basin, the two-way balance of RVA and FRIC suggests an inertio-frictional balance in the inner layer:

$$
\delta_{M}^{\star}=\sqrt{\frac{\delta_{M}}{\delta_{I}}} \delta_{M} .
$$

Using $\delta_{M}^{\star}=0.03$ taken approximately from the figure, Equation 4.16 suggests $\delta_{I} \sim 0.3$. This value compares sensibly with the scale of the outer boundary layer in Figures $4.12 \mathrm{~b}$, c, and $\mathrm{d}$, using the zero crossing of the planetary advection (double-dashed) curve at $x=0.6$ as a reference location. Again this balance differs from that suggested by linear theory.

The vorticity balance suggests a widening of the inertial layer with decreasing latitude. As the parcels approach the equator, they are swept out into the interior by the eddy that resides there in the mean. The effect of this eddy is observed in the widening of the scale of the PVA term in Figure $4.12 b$, c, and d, and by the very rough scaling presented above. 

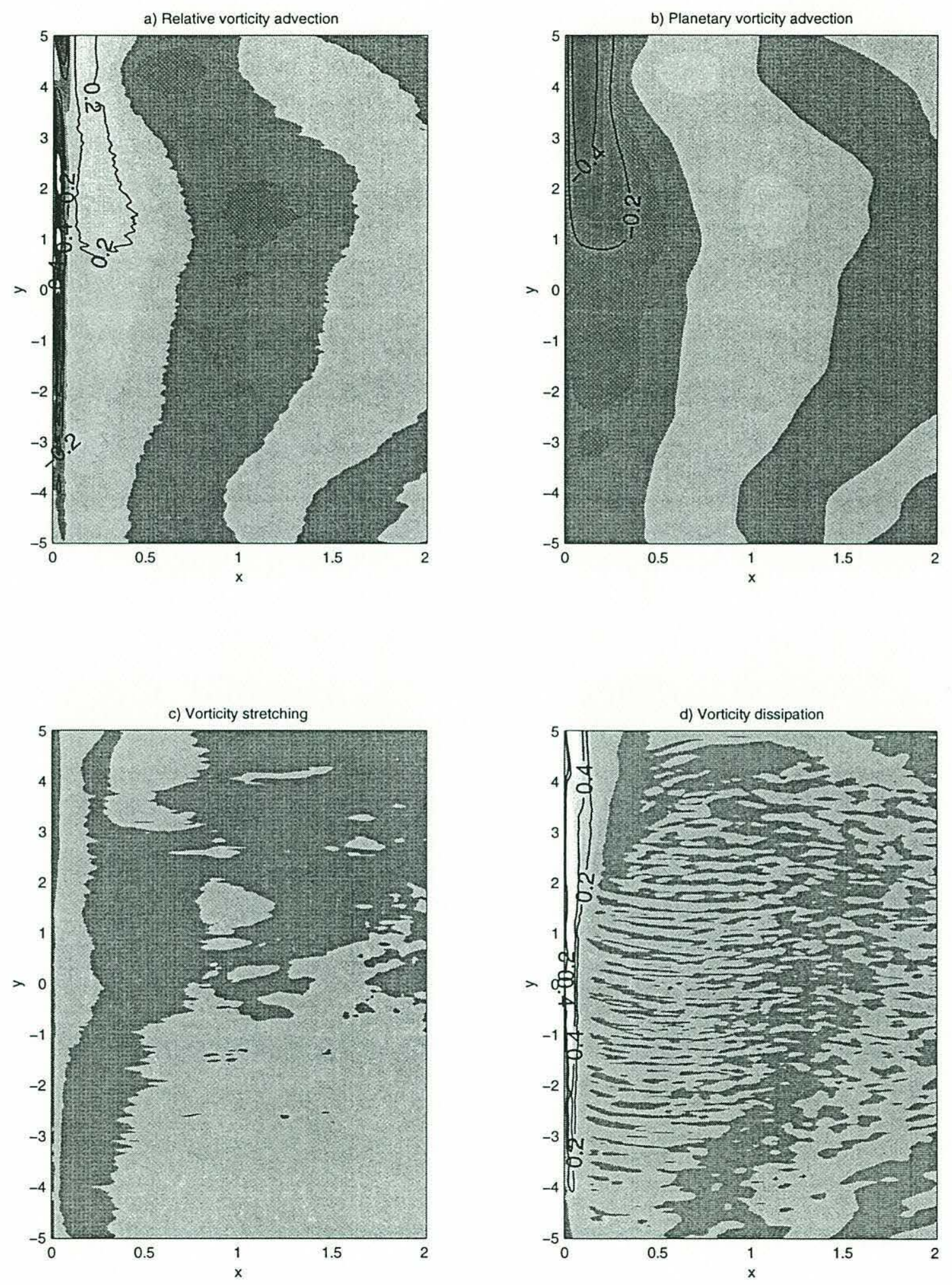

Figure 4.11: Plan view of the vorticity components in a subdomain for run U7 $(R e=350)$. Shades are every 0.1 with light values being positive, dark negative. In addition contours are placed at values of \pm 0.4 and \pm 0.2 to highlight the largest values. 

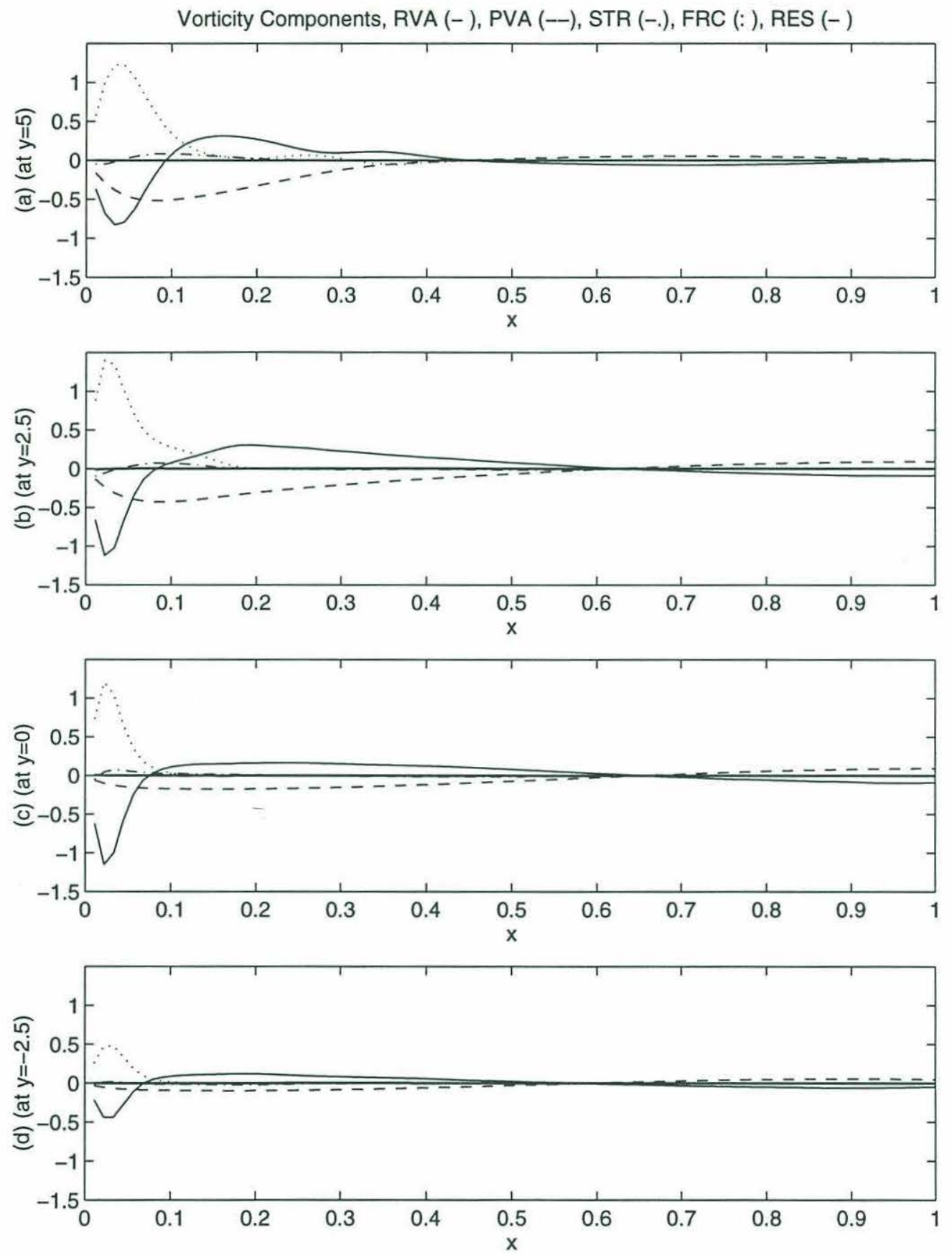

Figure 4.12: Zonal profiles of the different components of the vorticity equation, shown in Figure 4.11 at 4 different latitudes, (a) $y=5$, (b) 2.5 , (c) 0 , and (d) -2.5 . 


\subsection{Localized Upwelling}

A critical aspect of cross-equatorial flow is the determination of the actual crossequatorial mass-flux. In this work, it is specified through the upwelling distribution, $w_{\star}$. The results presented thus far concern a very specific system in which the upwelling distribution is constant, and therefore may be considered a potential weakness of this study. Indeed, although Stommel and Arons (1960a) considered a general form for the upwelling and directly examined both uniform and non-uniform distributions in their paper, the work is frequently referred to as pertaining to uniform upwelling only.

In an effort to demonstrate that the results presented here are robust to different upwelling distributions, I conduct an additional experiment that considers the most diametrically opposed forcing, a localized $w_{\star}$ (Equation 2.15). In this configuration, all the fluid entering in the northern hemisphere is destined to exit the basin through a sink entirely in the southern hemisphere. Thus all of the fluid entering the system is constrained to cross the equator. One limitation of this configuration is that the volume flux of fluid out of the sink must not be so large that the layer depth vanishes. Although this is not a problem at low Reynolds number, it can be significant as the volume of fluid transported through the system increases. As a result, the sink has a larger cross-sectional area than the source $(10 \times 10$ versus $5 \times 10$ deformation radii), and $w_{\star}$ is positive for all latitudes south of $y=-5$. A plot showing the upwelling distribution is presented in Figure 4.13.

The system evolves much as the system with distributed upwelling. There is an initial creation of a mound of fluid in the forcing region with subsequent Kelvin and Rossby waves setting up the interior circulation. Unlike the previous runs, the interior circulation is now absent, but there remains after the spin-up a residual western boundary current that carries fluid from source region to sink.

As in the uniform upwelling case, the system becomes unstable at higher Reynolds number, and eddies develop in the vicinity of the equator. In Figure 4.14 is shown the instantaneous velocity and height calculations from a $R e=75$ run. The accompanying 


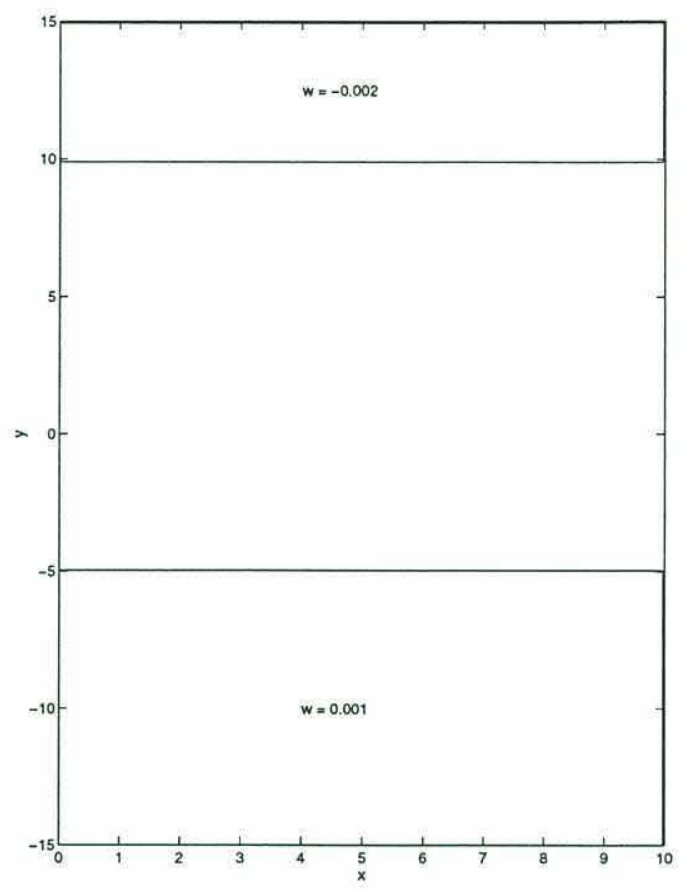

Figure 4.13: The upwelling distribution in the basin for run N2. The source is located in the northern hemisphere and the sink in the southern, with zero vertical mass flux in between.

potential vorticity field is presented in Figure 4.15. Eddies form immediately north of the equator, propagate steadily to the south, and dissipate vorticity along their path. In the mean, the boundary current widens at the equator (Figure 4.16). As before, this feature is associated with a recirculation, though displaced somewhat south of its location in the uniform upwelling case. Again, this recirculation is not indicative of trapped fluid, but rather of a location of enhanced eddy activity.

The vorticity flux streamfunction, $\Psi$, defined by Equation 4.13 takes a similar form as in the uniform upwelling case (Figure 4.17). Consistent with the requirement that all of the fluid passing through the system must cross the equator and therefore change its vorticity, most of the vorticity entering the subdomain of the basin through the northern boundary exits through the west (a small portion is fluxed back to the north in the counter-current that resides just east of the western boundary current). Calculating the index $E$ for this run (integrating only to $y=1.75$ where the integrated eddy flux is nearly constant) gives a value of $E=0.33$, which is slightly lower, but 


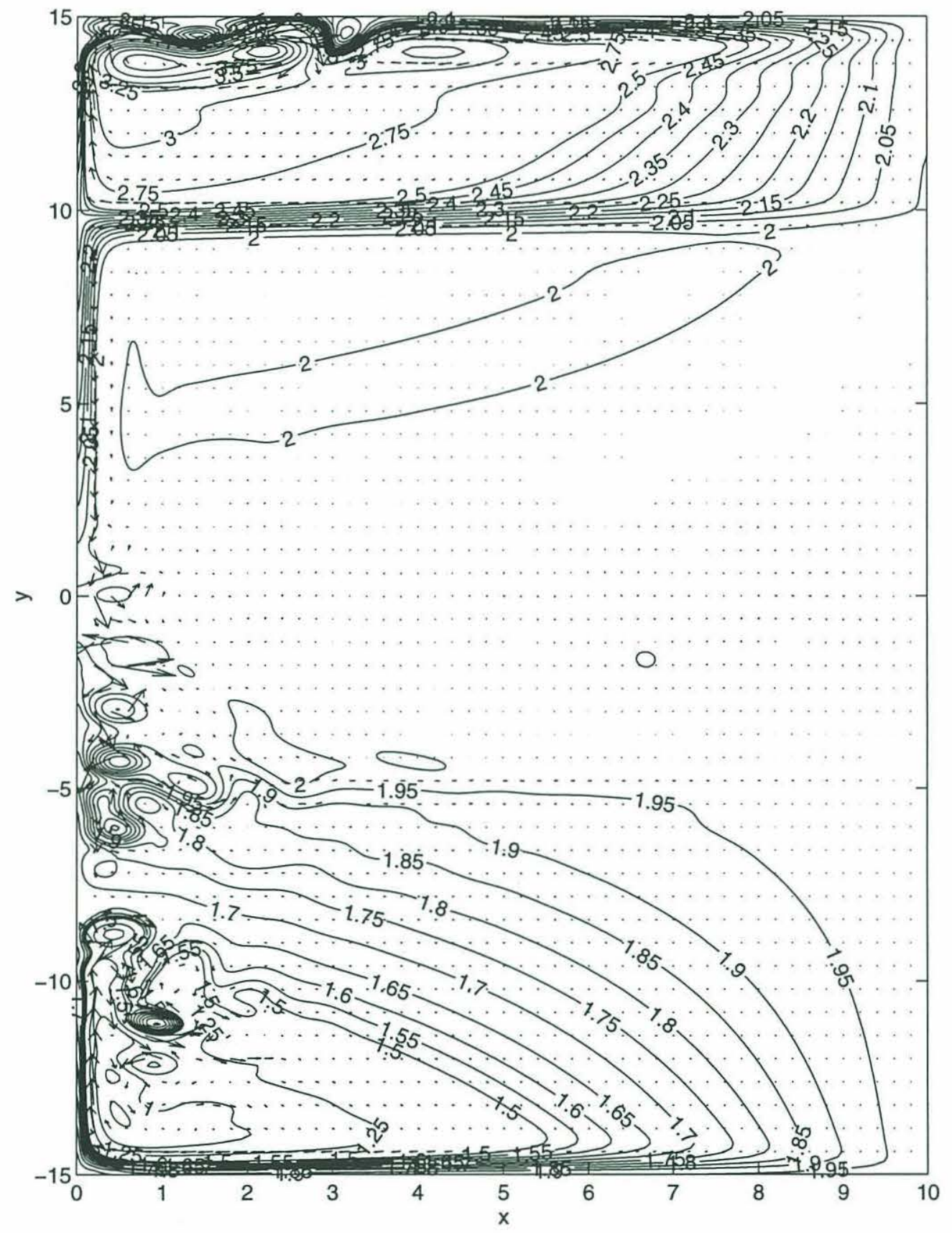

Figure 4.14: The velocity and height fields of a non-steady nonlinear run, N2 (Re = 75), and non-uniform forcing at time $t=3000$. The eddies develop quite near to the equator and propagate southward as in the intermediate Reynolds number cases with uniform upwelling. 


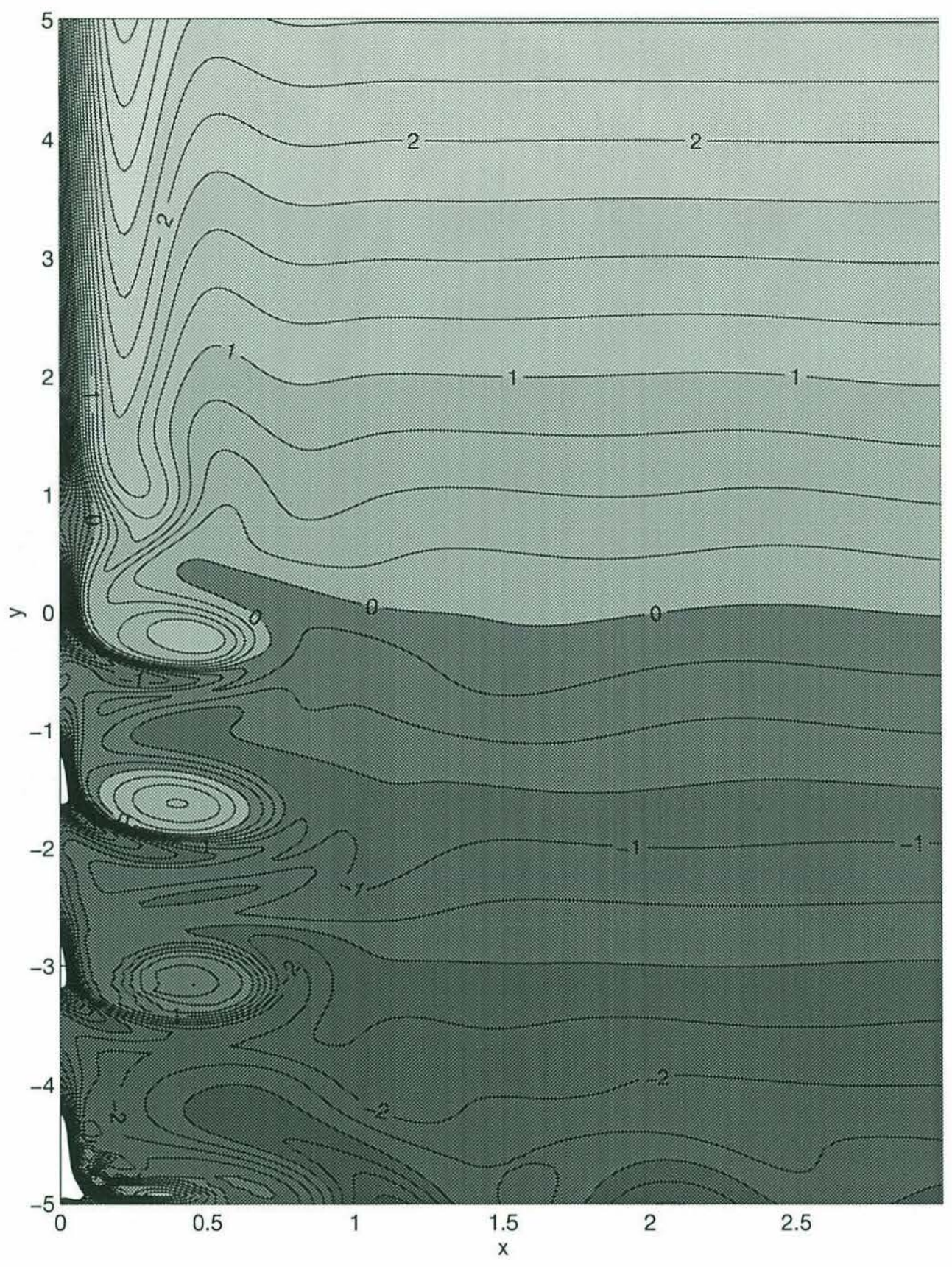

Figure 4.15: The potential vorticity field at time that accompanies Figure 4.14. 


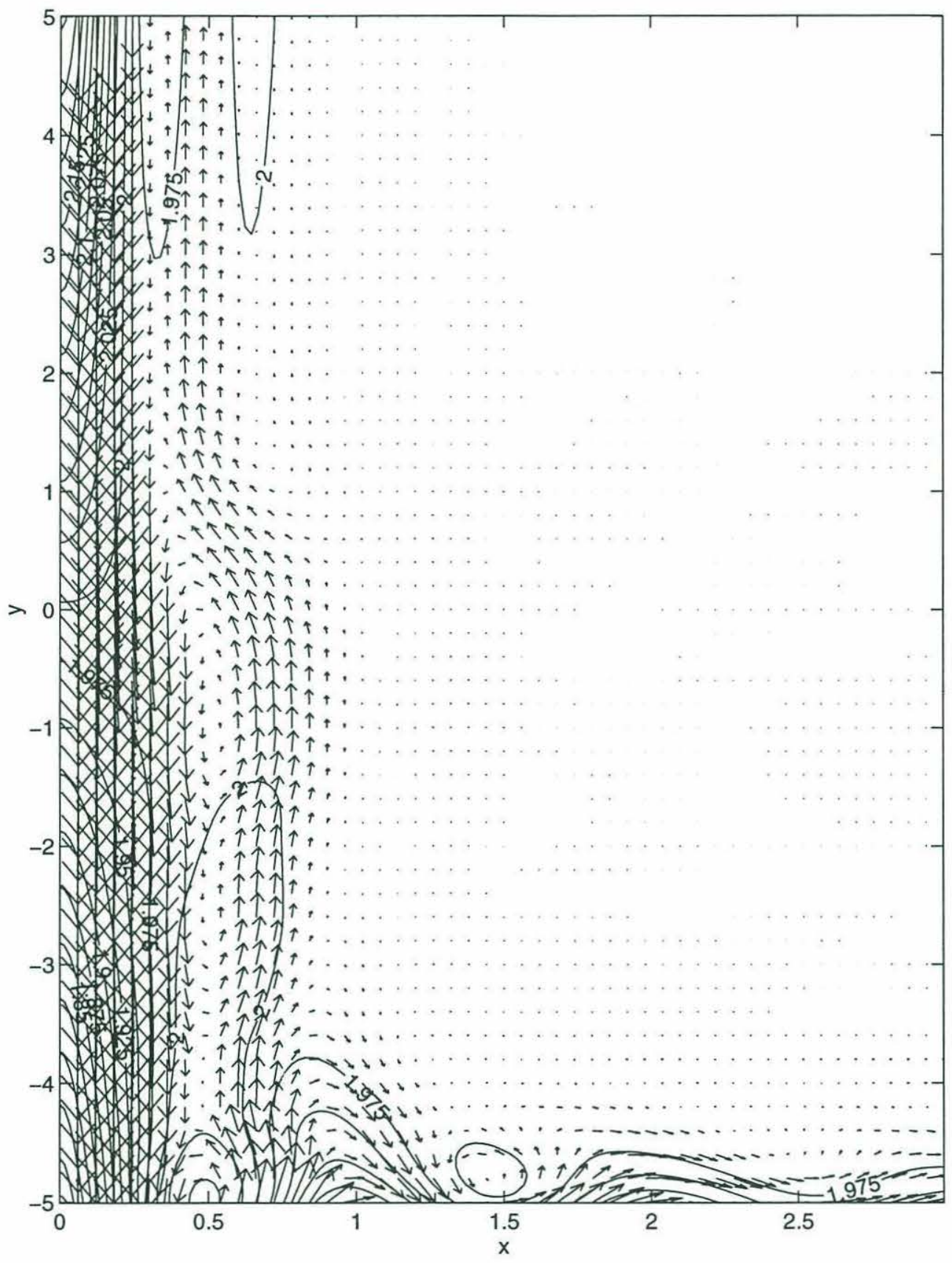

Figure 4.16: The time-mean velocity and height fields between $t=1000$ and $t=3000$ of run N2. 


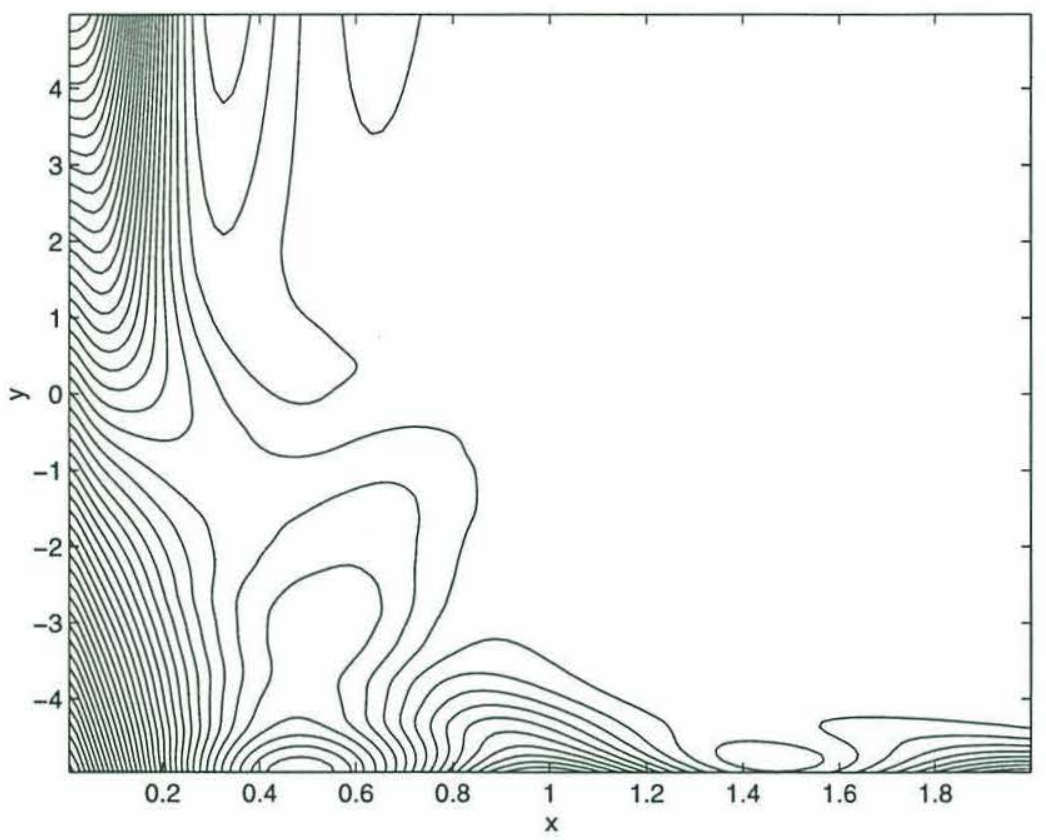

Figure 4.17: The vorticity flux streamfunction for run N2. Unlike the uniform upwelling runs, most of the vorticity entering the subdomain of the basin at $y=5$ leaves through the western boundary.

reasonably consistent with the behavior in Figure 4.10 and shown as the circle in that plot.

The details of the localized upwelling run are somewhat different from those of the previous runs. However, the basic mechanism is identical. The ratio of inertial to frictional boundary current increases with the Reynolds number. At a critical number, approximately $R e=30$, the system goes unstable, and the eddies provide the mechanism for the necessary lateral flux of vorticity to enable the conversion necessary for cross-equatorial flow. The upwelling specifies the mass that must flux across the equator. This section shows that regardless of the distribution of upwelling, there exists a sufficient eddy flux to allow that transport. 


\subsection{Free-slip Boundary Conditions}

Although float data in the equatorial Atlantic ocean indicate that the velocity parallel to the coast becomes small at the coast itself (Figure 1.3), I now consider the sensitivity of the results to this quantity by analyzing how the circulation changes with a different boundary condition. Specifically, I examine the the effect of a freeslip boundary. As can be expected from other studies (e.g., Marshall (1981) or the discussion in Ierley (1990)), this change alters the general circulation, the boundary layer structure, and the eddy fluxes from those using the no-slip condition. This section demonstrates that although some features do change with boundary conditions, the physical mechanism of eddy flux transport remains essential to the dynamics of nonlinear cross-equatorial flow.

Before considering the fully nonlinear case, it is instructive to revisit the steady, weakly nonlinear discussion of Section 4.1.2. The fundamental dynamical change to the system due to the new boundary condition is apparent in the integrated meridional vorticity flux (Equation 4.15c). With free-slip boundary conditions, the constraint that $v^{2}(x)$ vanish at the western boundary no longer applies, and the net meridional vorticity flux can be considerable, even at the equator. In turn, the relaxation of this constraint allows the system a greater meridional span over which to remove vorticity from the basin, and therefore the local westward vorticity flux is reduced from the no-slip system.

Consider the $R e=200$ run UFS whose upwelling is uniform, but the free slip condition is applied. Mean velocity and height fields are shown in Figure 4.18. The mid-latitude interior presents the familiar Stommel and Arons (1960a) eastward and poleward flow. Near the western boundary rests an intense southward flow on and immediately adjacent to the boundary, with an intense counterflow just to its east. The mean recirculation that had been present in the vicinity of the equator in the no-slip runs now rests quite far south in the southern hemisphere and is considerably elongated meridionally, compressed zonally. 


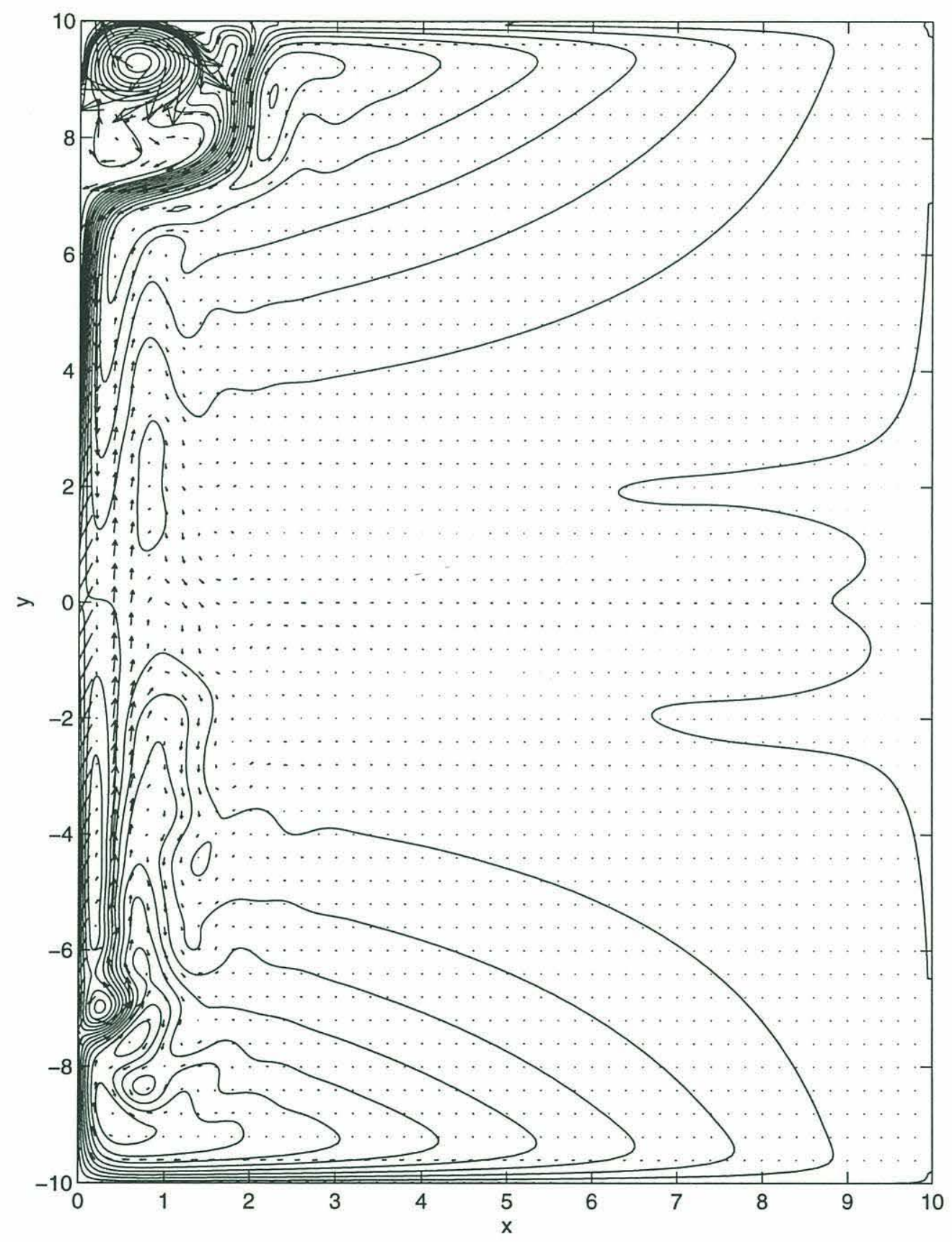

Figure 4.18: The time-averaged velocity field and height contours for the free-slip run UFS $(R e=200)$. 


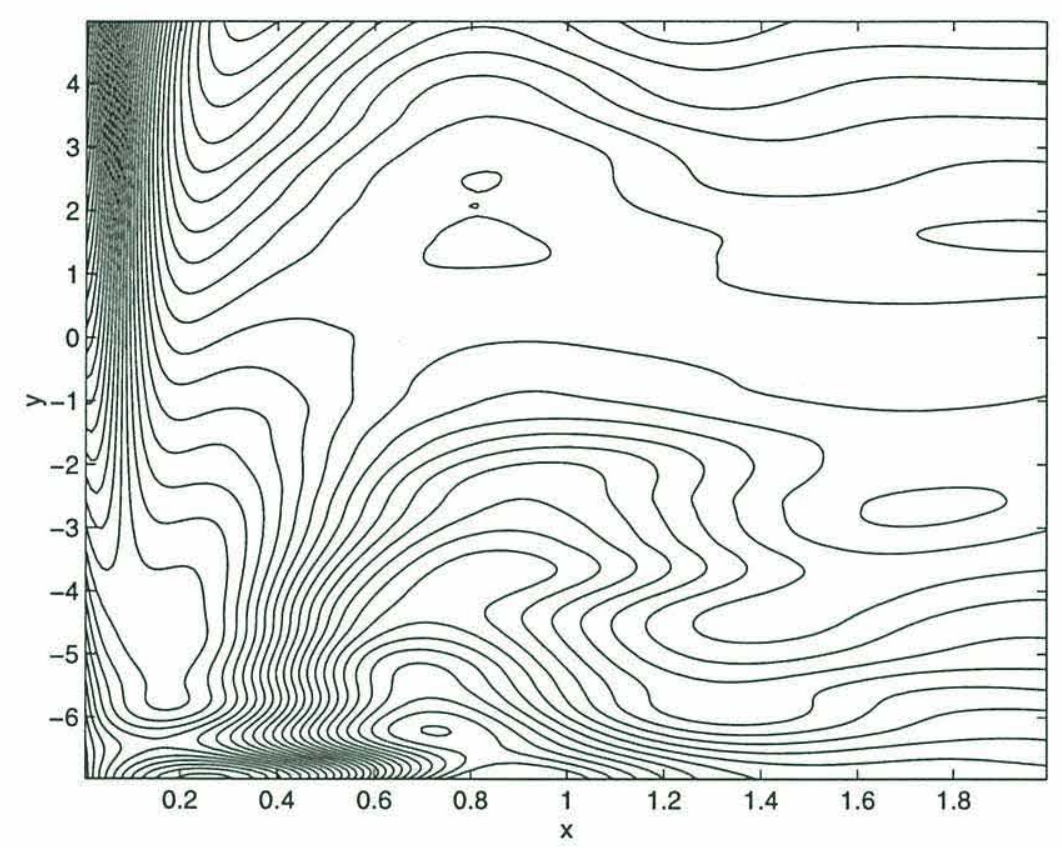

Figure 4.19: The vorticity flux streamfunction for a sub-domain of run UFS.

The vorticity flux streamfunction, $\Psi$, shows a large southward vorticity flux at the northern end of the subdomain, as before (Figure 4.19). However, now the streamlines pass smoothly across $y=0$, eventually turning westward out of the basin, but extending south beyond the equator several deformation radii.

Two integrated flux measurements are presented in Figure 4.20. In the upper panel is the net meridional vorticity flux, considerable at the northern edge of the domain and decreasing with latitude. This quantity vanishes at $y=-6$. In the lower panel is the net westward flux between $y=-6$ and $y=5$. Near to the boundary the total westward flux is primarily due to friction. To be sure, there is a small contribution to the westward flux in a turbulent boundary layer, visible by the small hump in the dot-dashed curve near $x=0.1$. This flux is strongest between latitudes $y=-4$ and $y=-7$, far south of the equator. Relaxing the constraint of no cross-equatorial meridional flux allows friction to dominate the boundary layer physics except in the vicinity of $y=-6$ where the zonally integrated vorticity flux is zero.

The significant role that friction plays in the boundary layer dynamics is also found 
a) Westward flux (mean(..), eddy(-.), fric(--), total(-), residual(heavy-))

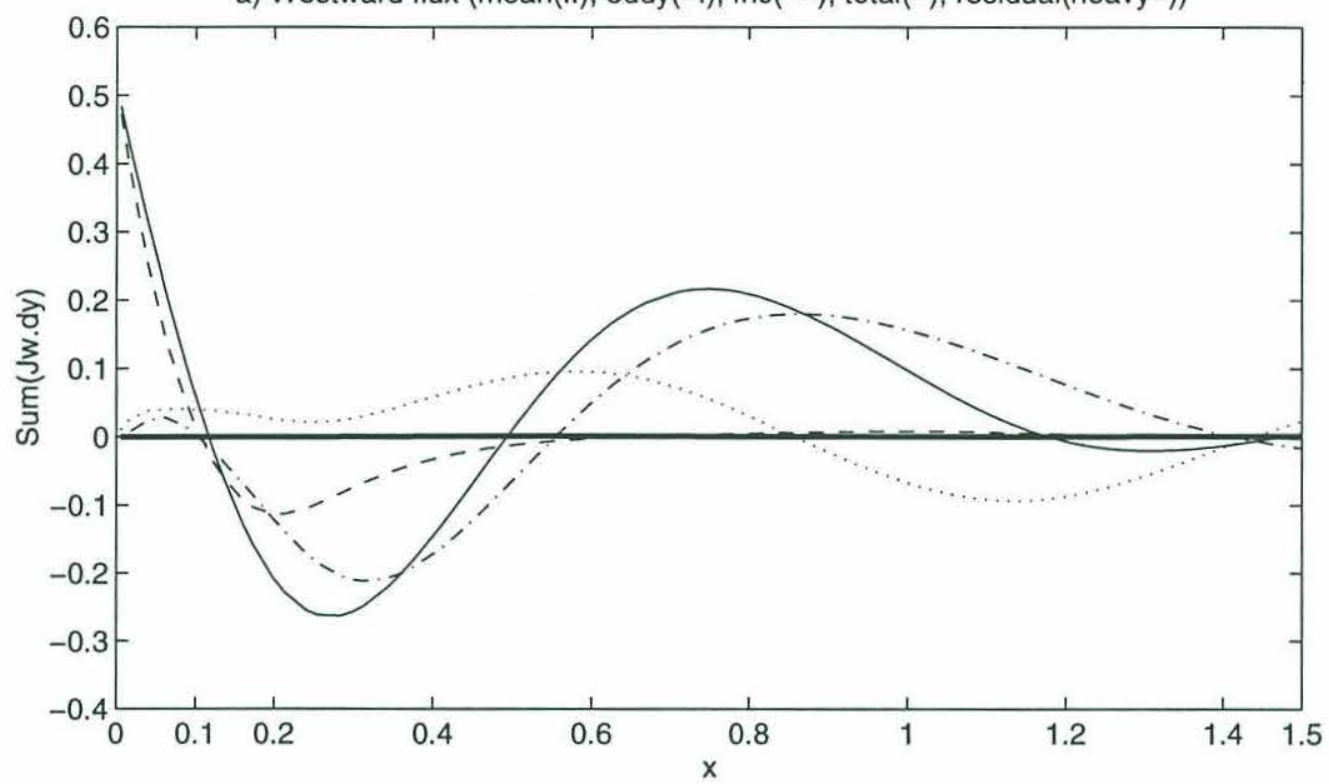

b) Southward flux

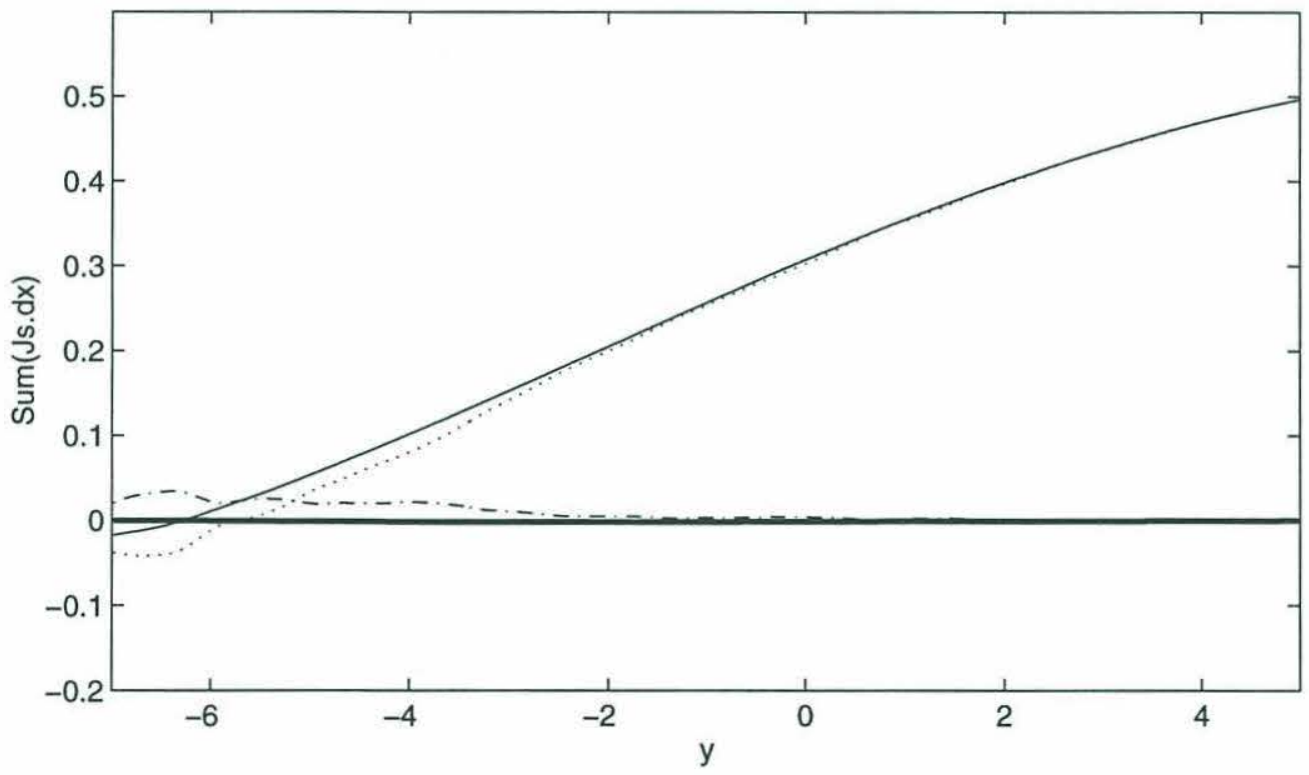

Figure 4.20: The integrated westward and northward vorticity fluxes for run UFS. The dashed curve corresponds to the frictional flux, the dotted to mean advection, the dot-dashed to the eddy flux, and the light solid curve is their sum. a) The integral extends from $y=-6$ to $y=5$, and the heavy solid curve is the sum of net fluxes through a sub-domain bounded by these latitudes, the longitude in the figure, and $x=2$. b) The integral extends zonally across the basin, and the sub-domain extends meridionally from the latitude in the figure to $y=5$. 
in the boundary layer structure. Shown in Figure 4.21 are the different components of the vorticity equation near the western boundary at 4 latitudes. In the northern portion of the basin at $y=5$, the boundary layer consists of two layers, an inner viscous sublayer of the inertio-frictional scale $\left(\delta_{M}^{\star} \cong 0.06\right)$ and an outer inertial layer. The meridional flow decays eastward from the western boundary and changes sign near $x=0.35$. Near the equator, the full boundary layer width has decreased and the meridional velocity changes sign near $x=0.2$, and the inner inertial layer has been subsumed into a wider frictional layer. This frictional balance extends south of the equator, and is shown for example at $y=-2.5$. Far south of the equator at $y=-6$, the boundary layer remains narrow, but the balance is more of a three term balance, both in the inner and outer portions of the southward flowing current. It is at this latitude that the structure of the boundary current most resembles the near equatorial balances of the no-slip case.

Although the transfer of vorticity to the boundary is largely achieved by the frictional boundary layer, eddies do become significant far south of the equator, and also in the interior. This interior process is represented in the strong peaks of integrated eddy flux in Figure $4.20 \mathrm{~b}$, eastward at $x=0.35$ and westward at $x=0.9$. Both functions of the eddy field are further highlighted in Figure 4.22 which shows the components of the $\mathbf{J}$-vectors due to the eddy field. Immediately adjacent to the boundary, the eddy field flux is negligible, except in a small region near the southern portion of the domain. Furthermore, the interior flux is easily visible in the strong convergence of vorticity near $x=0.6$. This convergence demarcates the eddy flux transfering vorticity to the northward-flowing counter-current from the western boundary current. Both the boundary current and the counter-current cross contours of mean potential vorticity. Associated with this crossing is a necessary vorticity flux divergence, predominantly due to the eddy field. Thus as in the no-slip case, eddies move vorticity to the boundary (in a small region in the southern hemisphere), and they are also responsible for the interior transfer from meridional flow to meridional flow and drive 

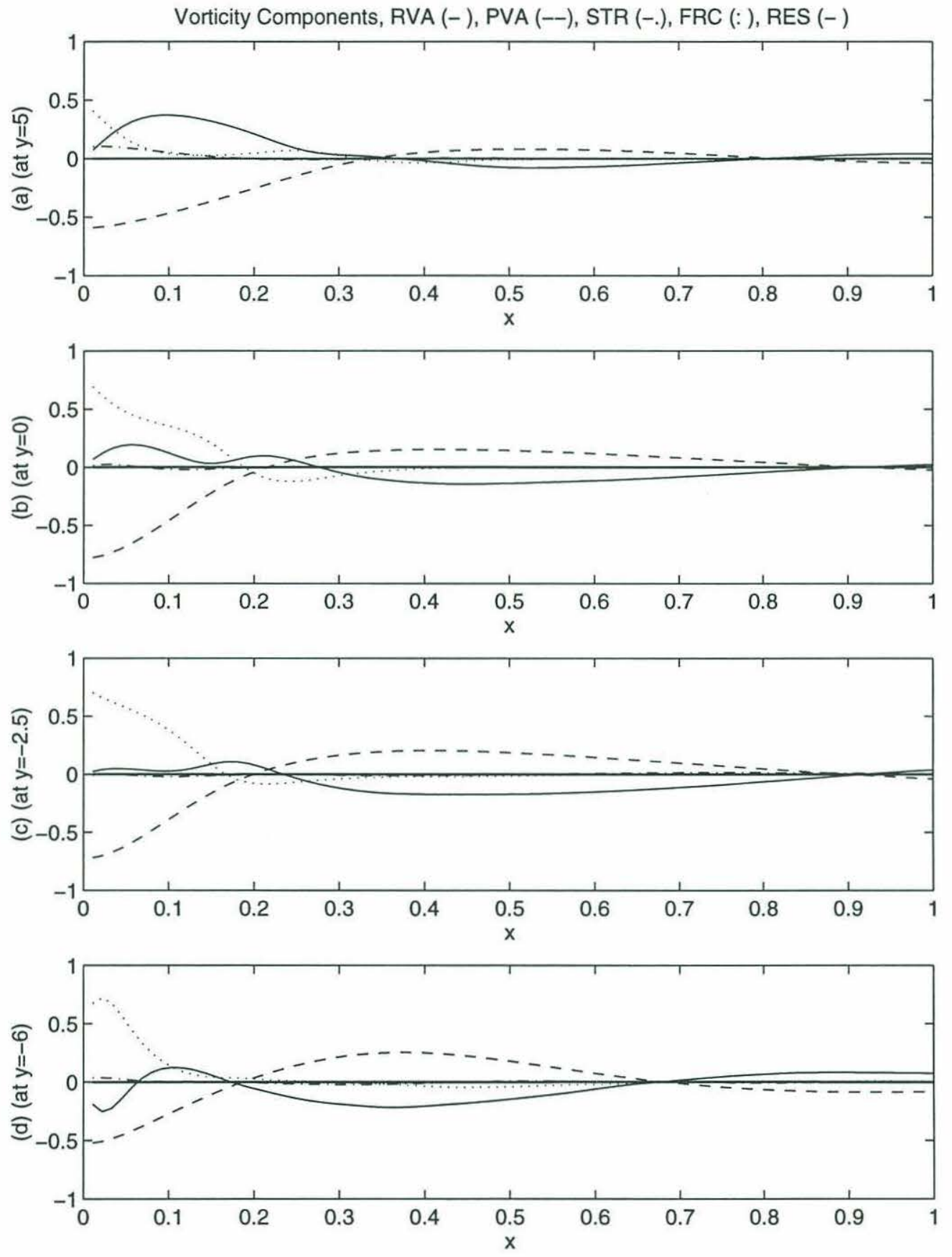

Figure 4.21: Different components of the vorticity equation at 4 latitudes in run UFS: (a) $y=5$, (b) $y=0$, (c) $y=-2.5$, and (d) $y=-6$. 


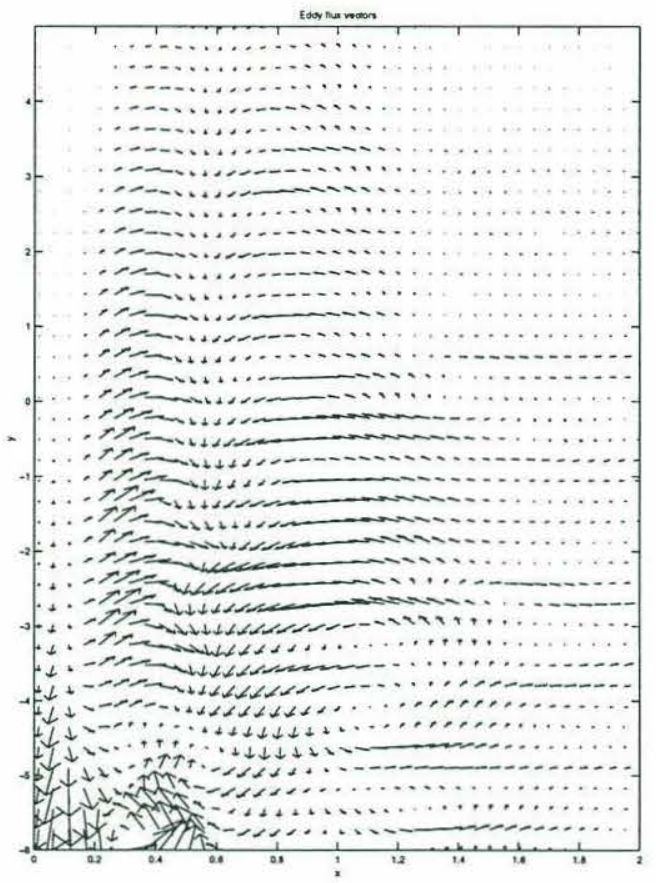

Figure 4.22: Eddy flux vectors for run UFS.

the recirculation.

It is clear from observations such as Figure 1.3 that the free slip boundary condition does not reflect nature; however, setting $v=0$ exactly on the boundary may also be inadequate, given the coarse resolution of even this model. It is important to draw from the above example the fact that although some features of the circulation have changed, fundamental aspects are similar. No longer constrained to flux vorticity out of the basin prior to reaching the equator, the system can maintain a frictional boundary layer over a meridionally extended portion of the basin, without invoking the eddy field. However, ultimately, the system confronts a similar constraint as in the no-slip case. At some latitude, the advective vorticity flux of the steady system must vanish. This special latitude is given by

$$
y \int_{0}^{x_{E}} v d x=\frac{1}{2} v^{2}(0)
$$

which must be internally determined by the system. In the above example, this equality is satisfied at approximately $y=-5.1$. It is this constraint which the steady 
system (frictional by definition) can not accomodate, and therefore requires the eddy field in this region to aid in the vorticity transfer. The boundary condition simply determines that latitude where the net meridional flux of vorticity must approximately vanish. For no-slip, it resides at the equator. For free-slip and strongly forced, it can be some distance south. Naturally, an intermediate boundary condition will produce an intermediate critical latitude, and the growth of an eddy flux at that location.

\subsection{Barotropic Limit}

One surprising result above is that the stretching term in the mean vorticity equation of Section 4.1.3 appears inconsequential in the dynamical balance of the western boundary current (refer again to Figure 4.12). The stretching term is an essential element of the shallow-water system, the critical feature that distinguishes it from purely barotropic models of the ocean. It is also the stretching that distinguishes the equator in the shallow-water model from all other latitudes. This fact can be seen from the inviscid quasi-geostrophic vorticity equation in which the stretching term is absent.

$$
\zeta_{t}+\mathbf{u} \cdot \nabla \zeta+v=0 .
$$

Without $f$ in the governing equation, the QG system considers only variations in $f$, not its absolute value. Returning to the vorticity calculations, the stretching term appears to be unimportant, but can not be neglected or the dynamics would behave quite differently.

A demonstration of this fact is found by examining the barotropic limit of the model, in which the boundary layer is small compared to the deformation radius. Figure 4.23 shows the instantaneous height and velocity field for model run UBar $(R e=100)$ at $t=500\left(\beta L_{D}\right)^{-1}$. Note that a quasi-steady state is achieved more quickly in this run because of the fast barotropic Rossby waves that return westward across the basin. The figure is dominated by long basin modes propagating westward 
in both hemispheres. However, in the vicinity of the western boundary current are again eddy features. However, unlike the baroclinic case, the variability develops immediately south of the forcing region, and extends steadily along the boundary at least beyond $y=-0.2$.

Thus when the boundary current is small compared to the deformation radius, the system is basically barotropic, and the model can not distinguish any unique equatorial physics. In the more baroclinic runs described earlier in the chapter, the equator clearly does play a role. Surprisingly, from the time-mean calculations, thickness advection appears to be small in the mean vorticity balance of the western boundary current. Of course, stretching is important to the interior mid-latitude balance, but this reflects the linear effect of the forcing, and not the maintenence of the height field fundamental to the shallow-water model.

It is possible that the the system recognizes the equator through the time-dependent stretching term, $\frac{f}{h} \frac{\partial h}{\partial t}$ which vanishes in the time mean. Indeed it is found in Chapter 5 that this term is essential to the development of eddies preferrentially in the vicinity of the equator. In the time-dependent model, this form of stretching could contribute to the vorticity balance at high latitudes, where $f$ is large, and the system is most baroclinic. Near the equator, $f$ is weak, and the effect of relative vorticity advection may be more dominant. However, cursory examination of instantaneous fields in the strongly nonlinear, $R e=500$ run, shows little correlation between the time evolution of vorticity and this stretching term.

It is important to remember that the Kelvin waves, so vital in the development of the circulation, require the presence of stretching term. From the strong variability that exists in the equatorial zone in Figure 3.10, it appears that these waves, and therefore the stretching term, may also play an important role contributing to the quasi-steady state as well, though I have not analyzed this aspect of the model. It remains an interesting question how exactly the change in sign of the Coriolis parameter influences the dynamics of the vorticity balance. 


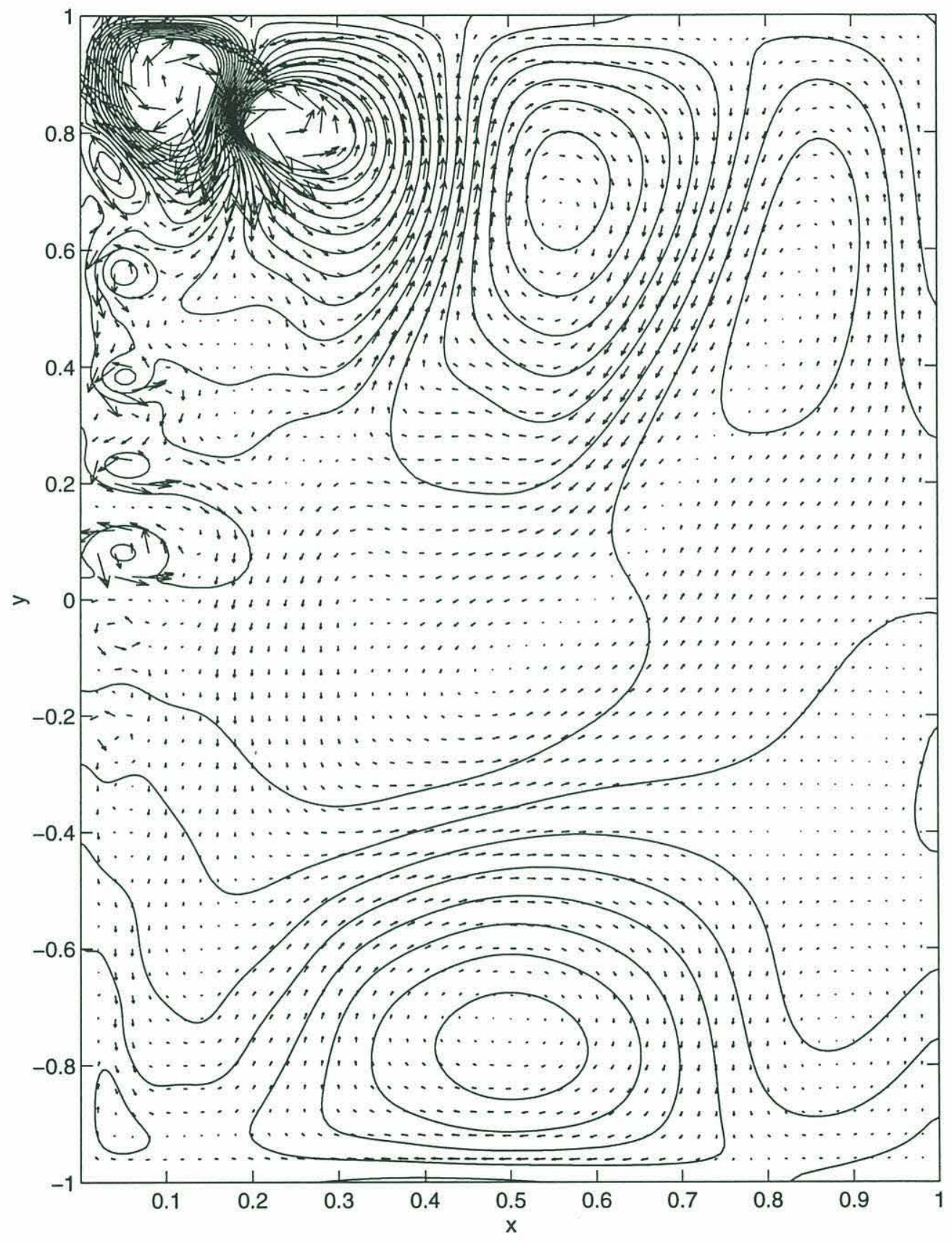

Figure 4.23: Instantaneous height contours and velocity quivers for the barotropic model run $\mathrm{UBar}(R e=100)$ at $t=500$. Notice that the variability in the western boundary current forms just south of the intense forcing region in the northwestern corner. 


\subsection{Summary}

Model integrations of Chapter 3 provide a qualitative description of the behavior of fluid in cross-equatorial flow under a range of conditions. The distribution of upwelling sets the volume flux of fluid that must cross the equator, and the system responds by creating an eddy field as the ratio of the volume flux of fluid to the viscosity increases. The formalism of the $\mathbf{J}$-vector analysis introduced in this chapter reveals and quantifies the dynamical role of the eddy field that appears in the numerical experiments. The eddies serve to transfer vorticity between inertial and frictional portions of the boundary current, enabling the potential vorticity transformation that permits the flow. The strength of the turbulent boundary layer increases with the Reynolds number perhaps without bound and at least to $R e=1000$. The behavior of the system is quite robust to different upwelling configurations, and though the circulation changes considerably using free-slip boundary conditions, the eddy field again plays a dynamical role in its vorticity budget. In the vicinity of the equator, the structure of the no-slip uniform upwelling boundary current is divided into two portions, an outer inertial boundary layer, and an inner layer obeying an inertioviscous balance.

The analysis above reflects its Eulerian perspective. It states how vorticity moves in the frame of reference of the basin. Speculating on the Lagrangian behavior of individual fluid parcels provides an alternative description of cross-equatorial flow. In strongly nonlinear flow, a parcel travels southward within the western boundary current, its vorticity is partially conserved, only weakly modified through a dissipative process. Near the equator, this parcel is swept into an intense cyclonic structure and immersed in a sea of eddies having both positive and negative senses of circulation. As the eddy sweeps past these eddies, and importantly, also past the boundary, it inevitably passes through narrow zones of intense velocity shear, where the vorticity is transferred, from parcel to parcel, and ultimately, from parcel to boundary. The path of any given parcel is extremely complicated and difficult to model, and the trajectories 
of neighboring parcels diverge rapidly once they enter the turbulent region. Once the parcel has transferred all of its anomalous vorticity, it can merge smoothly with a relatively weak interior flow, either in the northern or southern hemisphere, where the dynamics are more linear. 


\section{Chapter 5}

\section{The Tropically Enhanced}

\section{Instability of the Western}

\section{Boundary Current}

\subsection{Introduction}

Central to the discussion of cross-equatorial flow in the deep ocean is the development of the eddy field that arises as the system becomes increasingly nonlinear. Associated with these eddies is the necessary transfer of vorticity between different parts of the boundary current, which allows fluid parcels to modify their potential vorticity along their meridional journey. The existence of these eddies and their dynamical role are now well-established by the preceding experiments, but their cause is not explained. The goal of this chapter is to analyze the instability of the tropical western boundary current and explain the mechanism responsible for the eddy generation.

Strong eddy activity in the tropical oceans is not purely a numerical artifact but is also found in nature. As mentioned in the Introduction to the thesis, many observations indicate that the equatorial oceans are highly time-dependent regions. Although observations are insufficient to determine whether the region near the western bound- 


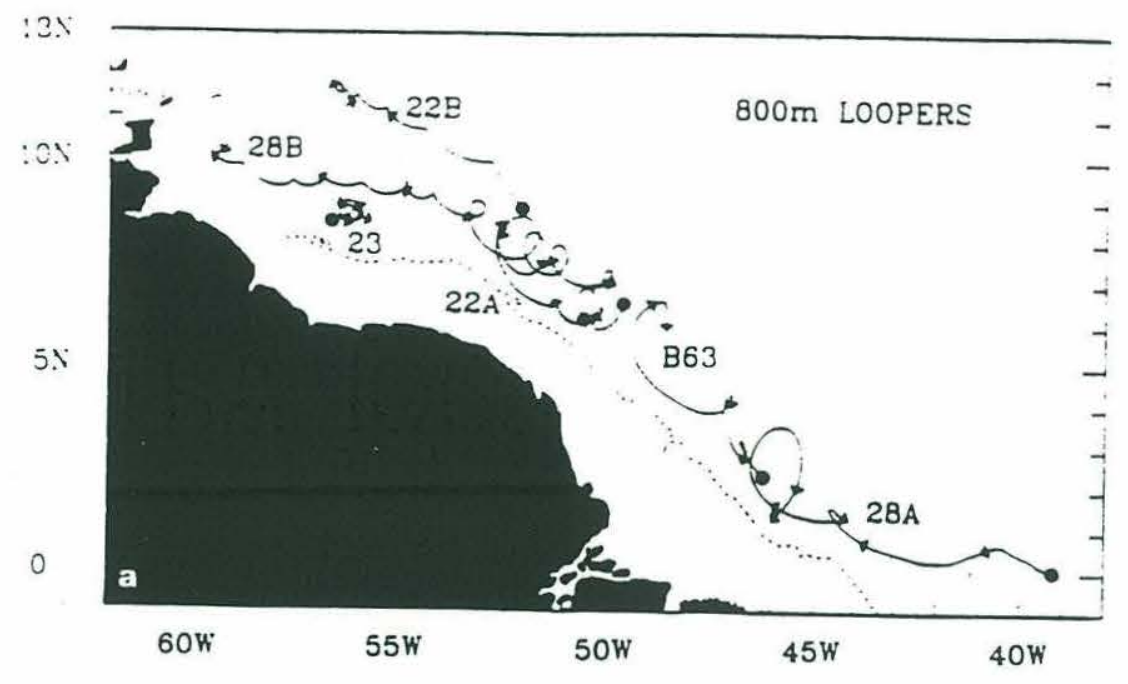

Figure 5.1: A collection of "looper" float trajectories at 800 meters in the Atlantic. Notice the development of eddy structures just north of the equator. This figure is reproduced from Richardson and Schmitz (1993).

ary in the tropics is in nature more eddy rich than at mid-latitudes, SOFAR float measurements in the Atlantic (Richardson and Schmitz (1993)) present a particularly compelling example that suggests this possibility. Shown in Figure 5.1, is a set of "looper" trajectories at 800 meters depth revealing the northward propagation of eddies along the boundary in this region and, potentially, their generation as well.

Close examination of the eddy formation process in the numerical model reveals that the phenomenon is dependent on both the Reynolds number of the flow and also on latitude. The eddies begin to develop for nonlinear flows with $R e>R e_{c}$, where the Reynolds number is defined by global basin parameters according to Equation 2.23. In addition, the features form preferentially near the equator in the no-slip case. This behavior is illustrated by the series of numerical experiments shown in Figure 5.2. In all three cases, the source is located at the northern end of the domain and the sink in the southern, and the basin has dimension, $L_{x}=3$ and $L_{y}=20$ though the figures show only a fraction of this region to concentrate on the unforced central region. The system is spun up from rest to a steady or quasi-steady state. In Figure 5.2a, a western 
boundary current carries fluid laminarly from the source region across the equator to the sink region. Thus the flow is stable at this Reynolds number $(R e=25)$. In Figure $5.2 \mathrm{~b}$, the $R e=50$ and the western boundary current is unstable, and eddies develop. The coincidence between their formation and the equator is striking and unmistakable. Finally, Figure 5.2c presents a run at $R e=50$ that does not cross the equator. Its central latitude is at $y=10$, and the flow is stable. It is the combination of a high Reynolds number and a constraint to cross the equator that stimulate the observed eddy field.

Thinking generally about the stability of a viscous shallow-water tropical system suggests three mechanisms that could be important. The most obvious candidate is horizontal shear instability, which has an extensive history dating back at least to Rayleigh (1880) (see discussion in Drazin and Reid (1981)) In this process, the velocity profile of the mean field is unstable to small disturbances. A sufficient condition for the flow to be stable is the absence of a potential vorticity extrememum in the flow profile anywhere in the domain. As a result, this process is sometimes referred to as an inflection point instability. Shear instability does not rely on the presence of friction. To the contrary, viscosity that is present in the system acts as a stabilizing force which can completely suppress an instability if sufficiently strong.

The second possibility is viscous instability. This mechanism has been suggested in studies of the mid-latitude western boundary current by Ierley and Young (1991) and Cessi et al. (1993). The physics of this instability is fundamentally the same as above. However, the basic flow (usually) has no potential vorticity extremum in the domain and is therefore stable to perturbations in the absence of friction. Without viscous forces, the Reynolds stresses $\overline{\left(u^{\prime} v^{\prime}\right)}$ vanish owing to a precise phase relationship between the different velocity components. As a result the disturbance can not extract momentum from the mean flow. With the addition of viscosity, this phase relationship is broken in a critical layer residing in the high shear zone adjacent to the boundary, enabling the Reynolds stresses to transfer momentum to the perturbation. An example 

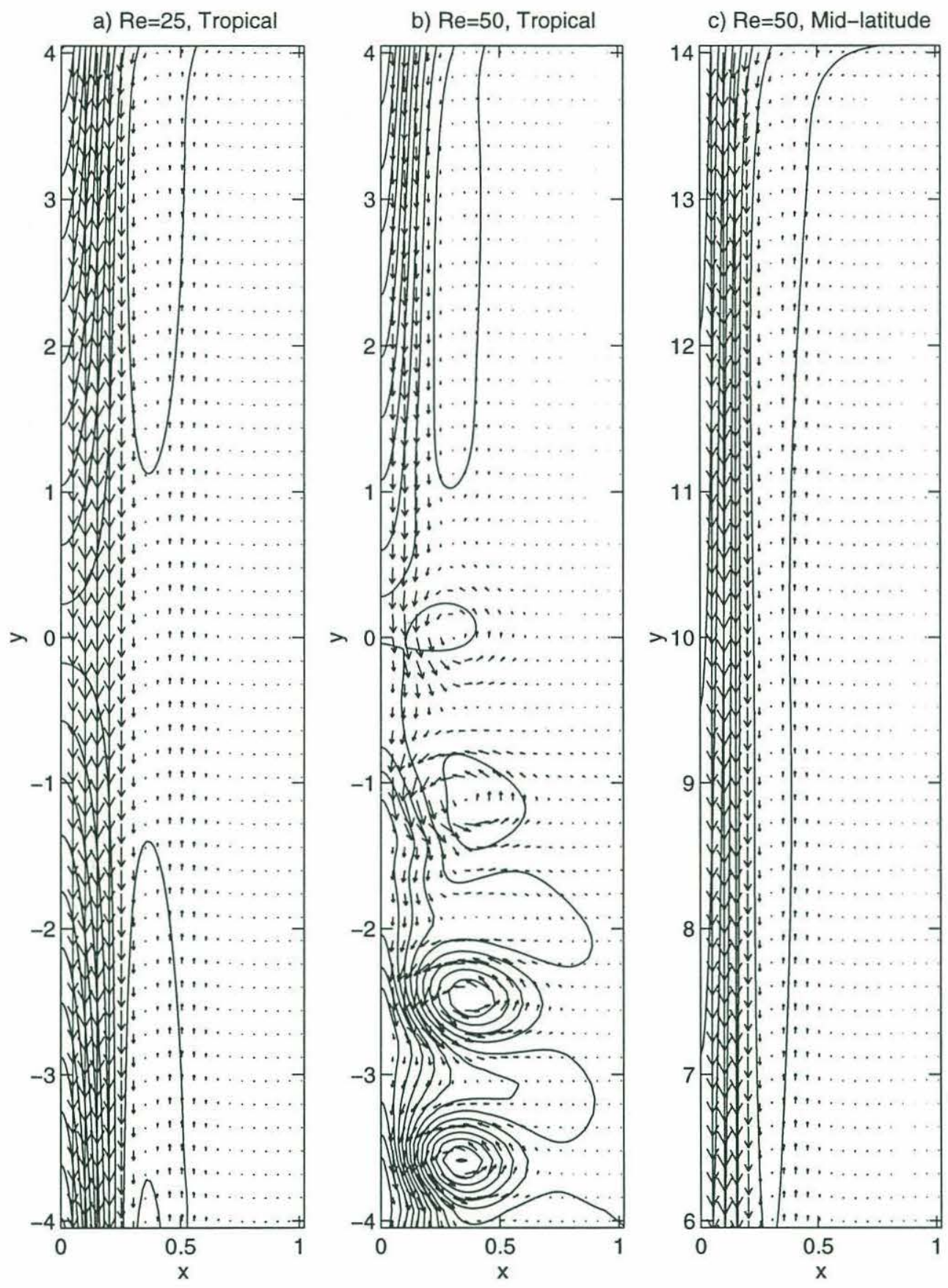

Figure 5.2: Velocity and height fields in subdomain of basin with source in the north and sink in the south at $t=1000\left(\beta L_{D}\right)^{-1}$ : (a) $R e=25$ and central latitude is the equator; (b) as in (a) but with $R e=50$; (c) as in (b) but with central latitude at $y=10$. 
of viscous instability is the plane Pouseille flow which has a parabolic profile. With no inflection point, the flow is stable to shear instability, but in real flows at very high Reynolds numbers $(\operatorname{Re}=5780$ (Kundu (1990)), the system undergoes a transition to time-dependent motion.

A third possibility is that the flow is inertially unstable, a process more directly related to the rotation of the system than horizontal shear instability. A familiar example is of Taylor-Couette flow in which two concentric cylinders with fluid between them rotate differentially. It can be shown (e.g., Kundu (1990)), that when the square of the circulation of fluid decreases with radius, the system becomes unstable and horizontal rolls develop to transfer momentum laterally. Since this process depends on the centrifugal acceleration of the fluid parcels, it is also called centrifugal instability. In geophysical contexts in which the Coriolis parameter arises, the necessary condition for instability in a highly idealized flow is that

$$
f q<0 .
$$

When the potential vorticity, $q$, of the flow is of the opposite sign as the Coriolis parameter, as happens in inertial cross-equatorial flow, the system becomes unstable. The theory applies to inviscid, parallel flow. In a real fluid, friction damps the disturbance as in shear instability.

In nature, other instabilities are possible, such as baroclinic or Kelvin-Helmholtz instability. However, these depend on the vertical velocity shear, and therefore are absent in a shallow-water model. Furthermore, baroclinic instability is not likely for mean flows with horizontal scale small with respect to the deformation radius (Pedlosky (1987a)) as is true for the western boundary current at the equator. As a result, I focus only on the three mechanisms above.

Determining the physics that drives the equatorial instability is not a pedantic quest. Its importance rests in the generality of the eddy formation process and the dynamical significance attributed to the eddy field in the previous chapter. If eddies develop only as a result of the details of the frictional parameterization, as suggested 
by the possibility of a viscous instability, then their role in transporting vorticity to the western boundary current is also quite fragile, potentially just an artifact of the particular model chosen. On the other hand, if the western boundary current is found to be unstable to the more robust shear instability, then the conclusions drawn previously are more general and are likely to apply to the ocean and to any model that produces a physically realistic western boundary current. It is in fact difficult to state absolutely which process is fundamentally responsible if, as is the case, all the elements for each instability are present. However, I will show that the likely process at work is classical shear instability of the western boundary current that is triggered preferentially at the equator because the maximum in the deformation radius occurs at that latitude.

\subsection{Formulation of the Problem}

The stability of the tropical western boundary current is assessed using the standard techniques of linear stability analysis, as found for example in Pedlosky (1987a) or Drazin and Reid (1981) along with several approximations. In particular, the western boundary current is idealized to be purely meridional and latitude-independent and the effect of the gradient in the planetary vorticity is neglected in the perturbation.

The shallow-water equations in dimensional form are recast

$$
\begin{aligned}
u_{t}+\mathbf{u} \cdot \nabla u-f v & =-h_{x}+A_{H} \nabla^{2} u+\Xi \\
v_{t}+\mathbf{u} \cdot \nabla v+f u & =-h_{y}+A_{H} \nabla^{2} v+\Upsilon \\
h_{t}+\mathbf{u} \cdot \nabla h+h \nabla \cdot \mathbf{u} & =-w_{\star}
\end{aligned}
$$

where additional body force terms $\Xi(x, y)$ and $\Upsilon(x, y)$, whose utilility will become evident below, are included in the momentum equations. The dependent variables are decomposed into time-mean and perturbation quantities,

$$
u(x, y, t)=\bar{u}(x, y)+u^{\prime}(x, y, t)
$$




$$
\begin{aligned}
& v(x, y, t)=\bar{v}(x, y)+v^{\prime}(x, y, t) \\
& h(x, y, t)=\bar{h}(x, y)+h^{\prime}(x, y, t) .
\end{aligned}
$$

For the predominantly meridional shear flow of a western boundary current, the basic state is idealized to be parallel flow that is only a function of longitude:

$$
\bar{u}=0 ; \quad \bar{v}=\bar{v}(x) ; \quad \bar{h}=\bar{h}(x) .
$$

Substituting Equations 5.4 and $5.3 \mathrm{a}-5.3 \mathrm{c}$ into the set $5.2 \mathrm{a}-5.2 \mathrm{c}$ yields a new set of equations in terms of both the mean and perturbation quantities. Since, linear stability theory models the initial development of infinitesimal disturbances to a basic state, the equations may be examined at different orders of the amplitude of the disturbance. To leading order, only terms independent of the prime variables remain,

$$
\begin{aligned}
f \bar{v} & =-g^{\prime} \bar{h}_{x}+\Xi \\
0 & =A_{H} \bar{v}_{x x}+\Upsilon .
\end{aligned}
$$

Equations $5.5 \mathrm{a}$ and $5.5 \mathrm{~b}$ are in essence a redefinition of the basic state. Body force terms, $\Xi$ and $\Upsilon$, remain in the equations at this order, and they represent the artificial but necessary forces to balance the idealized mean flow and maintain a purely meridional, latitude-independent basic state. Their functional form depends exactly on the chosen form for the mean flow used. For example, for a desired basic state velocity profile, $\bar{v}^{0}(x)$ and $\bar{h}^{0}(x)$,

$$
\begin{aligned}
\Xi(x, y) & =f(y) \bar{v}^{0}(x)+g^{\prime} \bar{h}_{x}^{0} \\
\Upsilon & =-A_{H} \bar{v}_{x x}^{0} .
\end{aligned}
$$

Alternatively,

$$
\frac{\partial \Xi}{\partial y}=\beta \bar{v}^{0} .
$$

Thus the forcing, $\Xi$, satisfies a vorticity balance and maintains the desired meridional velocity field, and $\Upsilon$ balances any viscous dissipation of that flow. The vertical mass 
flux, $w_{\star}$, is absent in the equations, reflecting the fact that a local vertical mass flux can not drive a steady, latitude-independent basic flow.

Moving to the next order retains terms linear in the perturbations

$$
\begin{aligned}
u_{t}^{\prime}+\bar{v} u_{y}^{\prime}-f v^{\prime}+h_{x}^{\prime}-A_{H} \nabla^{2} u^{\prime} & =0 \\
v_{t}^{\prime}+\bar{v}_{x} u^{\prime}+\bar{v} v_{y}^{\prime}+f u^{\prime}+h_{y}^{\prime}-A_{H} \nabla^{2} v^{\prime} & =0 \\
h_{t}^{\prime}+\bar{h}_{x} u^{\prime}+\bar{v} h_{y}^{\prime}+\bar{h}\left(u_{x}^{\prime}+v_{y}^{\prime}\right) & =0 .
\end{aligned}
$$

The time evolution of the disturbance is determined by this set of equations in which the perturbation variables are coupled both to one another and to the basic state. The artificial body forces, $\Xi$ and $\Upsilon$, that drive the mean flow are absent in the perturbation equations. Thus their inclusion in the original physics has no effect on the instability of the system except in so far as establishing the mean field.

The coupled set of partial differential equations, 5.8a-5.8c, can be analytically simplified by making a stringent assumption. With the exception of the Coriolis parameter, $f=f(y)$, all of the coefficients of the dependent variables are functions of longitude only and not of latitude or time. If a local $f$-plane approximation is made and the Coriolis parameter is assumed to have a constant value, $f_{0}$, then a modal decomposition of the dependent variables is possible in $y$ and $t$. Let

$$
\begin{aligned}
\left(v^{\prime}, h^{\prime}\right) & =(V(x), H(x)) e^{i l(y-c t)} \\
u^{\prime} & =i l U(x) e^{i l(y-c t)},
\end{aligned}
$$

where $l$ is the meridional wavenumber of the disturbance and $c$ represents its phase speed. The wavenumber-weighting of the zonal amplitude function in Equation 5.9b merely simplifies the ultimate form of the mathematical problem and represents no fundamental difference between the different components. The method outlined here is similar to that of $\mathrm{Ni}(1996)$ who considered a zonal flow in a multi-layer, inviscid shallow-water model.

The $f$-plane approximation that allows this decomposition is a significant limitation of the present analysis as the variation of the Coriolis parameter is usually 
essential in tropical dynamical studies. A full treatment of the problem must include both the meridional variation of $f$ and the non-zonal nature of the basic flow. However, the full analysis is beyond the scope of this work, and as will be shown below, the simplification to a local $f$-plane calculation provides surprisingly good agreement with numerical results and does so in a desirably simple and understandable framework.

Using relations $5.9 \mathrm{a}$ and $5.9 \mathrm{~b}$, the system $5.8 \mathrm{a}-5.8 \mathrm{c}$ reduces to

$$
\begin{aligned}
-l^{2}(\bar{v}-c) U-i l A_{H}\left(U_{x x}-l^{2} U\right)-f V+H_{x} & =0 \\
\left(f+\bar{v}_{x}\right) U+(\bar{v}-c) V+\frac{i}{l} A_{H}\left(V_{x x}-l^{2} V\right)+H & =0 \\
\left(\bar{h}_{x} U+\bar{h} U_{x x}\right)+\bar{h} V+(\bar{v}-c) H & =0 .
\end{aligned}
$$

Equations 5.10a-5.10c can be re-written in the matrix form

$$
\mathbf{A} \psi=c \psi
$$

where

$$
\mathbf{A}=\left[\begin{array}{ccc}
\bar{v}+i l A_{H}\left(\frac{1}{l^{2}} \frac{d^{2}}{d x^{2}}-1\right) & \frac{f}{l^{2}} & \frac{1}{l^{2}} \frac{d}{d x} \\
f+\bar{v}_{x} & \bar{v}+i l A_{H}\left(\frac{1}{l^{2}} \frac{d^{2}}{d x^{2}}-1\right) & 1 \\
\bar{h}_{x}+\bar{h} \frac{d}{d x} & \bar{h} & \bar{v}
\end{array}\right]
$$

and

$$
\psi=\left[\begin{array}{c}
U \\
V \\
H
\end{array}\right]
$$

is the state vector. Equation 5.11 is a standard eigenvalue problem in which there exists a corresponding eigenvector, $\psi^{i}$, for each eigenvalue, $c^{i}$. From the modal representation of the perturbation amplitudes, stable modes have phase speeds either real or complex with negative imaginary component. Any eigenmode that has a positive imaginary component grows exponentially in time, at least until the perturbations reach finite amplitude.

Equation 5.11 gives the stability properties for meridional shear flow, of infinite zonal extent. To apply this formulation to the realistic shear flow of a western boundary current requires a finite domain, $x=\left[0, x_{E}\right]$, where the distance to the eastern 
boundary of the basin, $x_{E}$, is expected to be large compared to the scale of the shear flow itself. Boundary conditions at the solid meridional boundaries are no flux and no slip,

$$
\begin{aligned}
& U=0 \\
& V=0 .
\end{aligned}
$$

The full linear stability analysis even for this somewhat idealized flow as expressed by the matrix, A, reveals a complicated dependence of the state vector on the basic state and on the latitude, through the magnitude of the Coriolis parameter. It is not immediately clear how the stability of the system will change with latitude or with the mean flow itself. To gain further insight from the equations directly requires additional simplifications that are discussed in the next section.

\subsection{Inviscid Shear Instability}

Although Equation 5.11 can be discretized and analyzed as written for a variety of mean fields, it is useful to further reduce the problem first so as to isolate the different instability mechanisms. Setting the viscosity coefficient, $A_{H}$, to zero eliminates viscous instability from the system. The matrix, $\mathbf{A}$ then reduces to

$$
\hat{\mathbf{A}}=\left[\begin{array}{ccc}
\bar{v} & \frac{f}{l^{2}} & \frac{1}{l^{2}} \frac{d}{d x} \\
f+\bar{v}_{x} & \bar{v} & 1 \\
\bar{h}_{x}+\bar{h} \frac{d}{d x} & \bar{h} & \bar{v}
\end{array}\right],
$$

and the order of the system is reduced. The new eigenvalue problem is defined by,

$$
\hat{\mathbf{A}} \psi=c \psi .
$$

Only two boundary conditions are needed, and the obvious choice is that of no normal flow, Equation 5.14a. The no-slip condition, Equation 5.14b seems unphysical if the disturbance can not detect any dissipation. Thus friction is required to establish the mean flow profile, but its effect on the perturbation is neglected. 
The stability criterion for inviscid zonal flows on the $\beta$-plane in the shallow-water system has been examined by Ripa (1983). The case with $\beta=0$ corresponds to the present system. Stability is insured if (i)

$$
[\alpha-\bar{v}(x)] \frac{d \bar{q}}{d x} \geq 0
$$

and (ii),

$$
[\alpha-\bar{v}(x)]^{2} \leq g^{\prime} \bar{h}(x)
$$

for all $x$ and any $\alpha$. For the standard Munk boundary current as well as inertio-viscous profiles, condition 5.17 is generally violated assuming $\alpha$ in the range of the velocity.

The following section briefly describes the numerics involved in the solution of the eigenvalue problem. Section 5.3.2 determines and examines the stability of 5.16 using the inviscid formulation, $\hat{\mathbf{A}}$, and shows that the Munk boundary layer is in fact unstable to small perturbations, and that the shear instability growth rate is largest near the equator. Section 5.3.3 makes two additional approximations that allow a still simpler mathematical and physical interpretation of the phenomenon that more clearly illustrates the tropical enhancement of the instability.

\subsubsection{Numerics}

All eigenvalue equations analyzed are discretized on a stretched grid in $x$ with staggered variables in a manner similar to that described in Section A.2 for the timedependent model, except that for this western boundary problem, the stretching function is a simple exponential:

$$
x(i)=d\left(e^{\frac{s i}{N}}-1\right)
$$

where

$$
d=\frac{x_{E}}{e^{s}-1},
$$

$N$ is the number of grid points, and $s$ gives the degree of stretching. A value of $s=3$ was chosen to adequately enhance the resolution in the rapidly varying western 
boundary region without introducing excessive deformation of the grid. The results are slightly dependent on the number of grid points. All calculations were determined using a value of at least $N=100$, though even $N=32$ appears adequate to reveal the general behavior of the instability.

Generally, the matrix, A, is determined numerically, given the basic state and the latitude, and the eigenvalues, $c$, and eigenvectors, $\psi$, are determined using the eigenvalue solver, eig.m, which calls industry standard EISPACK routines, and is included in the basic distribution of Matlab. Checking the solutions using a different solver, sptarn.m, which applies a different algorithm to find selected eigenvalues produces virtually indistinguishable results. The numerical method for solution was checked against and agreed well with the asymptotic stability analysis of Lipps (1963) for a wall-free Bickley jet.

\subsubsection{Instability of the Munk Boundary Layer}

It is desirable to examine first a simple basic flow in which the fields are truly latitudeindependent. Any asymmetry that develops in the instability characteristics can then be attributed to the shallow-water system and not to changes in the potential vorticity structure of the basic state. I will examine later in Section 5.3.4 the instability of a more realistic, latitude-dependent flow field. The analytic solution to the Munk boundary layer provides a convenient basic velocity profile to begin the investigation:

$$
\bar{v}(x)=D \sin \left(\frac{\sqrt{3} x}{2 \delta_{M}}\right) e^{-\frac{x}{2 \delta_{M}}}
$$

where

$$
D=-\frac{2 S_{0}}{\sqrt{3} \delta_{M}} .
$$

Rather than use the geostrophically balanced height field, which varies with latitude, I set the height of the layer uniform:

$$
\bar{h}=1 .
$$




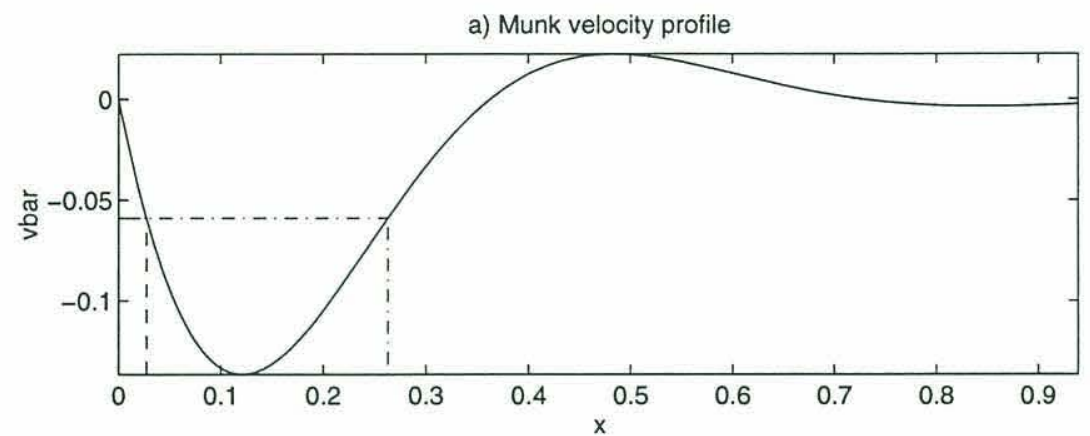

b) Potential vorticity gradient

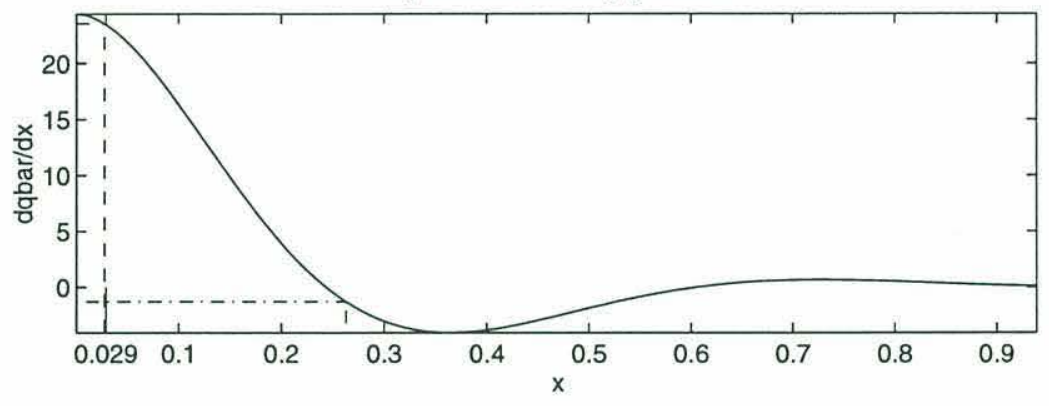

Figure 5.3: a) Velocity (in units of $c=\sqrt{g^{\prime} H}$ ) and b) potential vorticity gradient (in $\frac{\beta}{H}$ ) for the Munk boundary layer with $\delta_{M}=0.1$ and $S_{0}=-0.025$. Only shown to $x=1$, though $x_{E}=2$ in calculations below. Dot-dashed curve marks the intersection, $\bar{v}=0.059$, which equals the phase speed of the fastest unstable mode with $l=6$.

The quantitative adjustment for a geostrophically balanced height field for the weak velocities examined is small. The Munk layer is determined using $\delta_{M}=0.1$, and for a transport of $S_{0}=0.025$, consistent with the numerical model runs in Figure 5.2. Equation 5.16 is solved for the profile, 5.21 , a Coriolis parameter, $f=1$, and a reduced gravity, $g^{\prime}=1$. The basic state velocity profile is shown in Figure 5.3 along with the zonal gradient of the potential vorticity. The eastern boundary extends $20 \delta_{M}$, though only the westernmost $10 \delta_{M}$ is shown in the figure. The potential vorticity gradient passes through zero at multiple longitudes so the inflection point criterion is satisfied by this basic state.

Note that the scales of the basic states used in this chapter are identical to those of the rest of the thesis. Thus length scales are given in terms of deformation radii (i.e., wavenumbers in terms of inverse deformation radii), growth rates are scaled by $\beta L_{D}$, and phase speeds are relative to the gravity wave speed, $c=\sqrt{g^{\prime} H}$. 

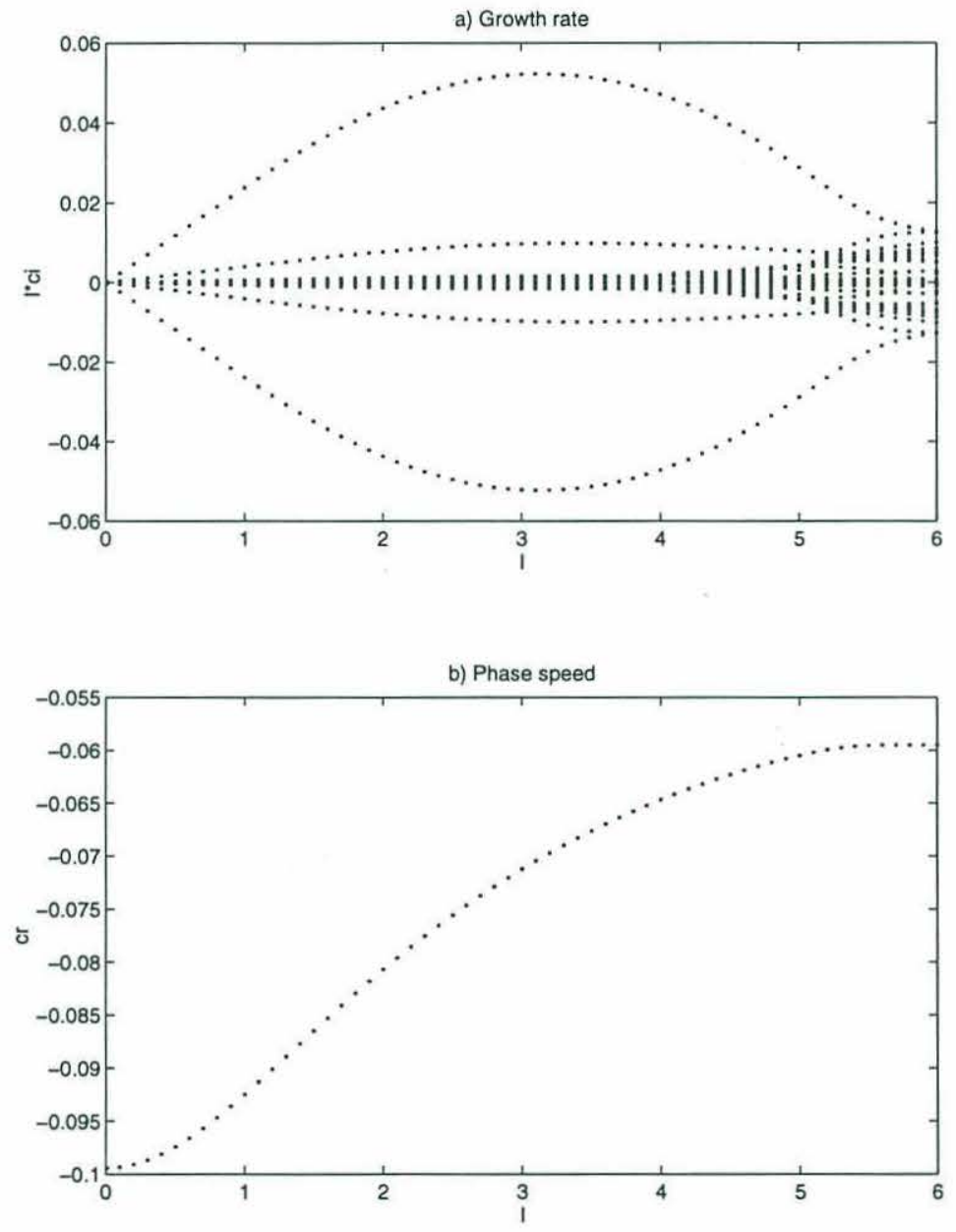

Figure 5.4: a) Growth rates as a function of meridional wavenumber for the velocity profile in Figure 5.3 in the inviscid shallow-water system. b) Phase speeds $\left(\sqrt{g^{\prime} H}\right)$ for the fastest growing modes in (a). 
The growth rate, $\operatorname{Im}(l c)=\omega_{i}$, of all eigenmodes of the system are shown in Figure $5.4 \mathrm{a}$ as a function of the wavenumber, $l$. The number of modes equals the dimension, $3 N$, of the system. Most modes are neutral, with zero imaginary component. They represent disturbances that are purely wavelike that pass through the system neither growing nor decaying. Positive and negative $\omega_{i}$ indicate growing and decaying modes, respectively, and the symmetry about $l c_{i}=0$ indicates that for each growing mode there exists a corresponding decaying mode. This feature is to be expected from the form of the matrix 5.15 in which all elements are purely real. The state vector, $\psi$, can be replaced by its complex conjugate, and the equation remains the same. Thus there is a symmetry between eigenvalues and their complex conjugates, and therefore also between growth and decay rates. Notice that this feature changes with the inclusion of friction, as in matrix, A, Equation 5.12.

In Figure 5.4a a few sets of unstable modes can be traced as a function of wavenumber. In particular, one pair of modes has the largest amplitude for all $l$, and they peak at a value of approximately $l=3.25$. This series and the additional sets of modes that appear within its envelope are the sets of modes that I consider to result from shear instability, and they are quite insensitive to the exact discretization and grid resolution. Notice that at high wavenumber there exist an increasing number of unstable modes of small amplitude. These are more fragile modes that depend more closely on the discretization of the system. While all unstable modes grow, it is the one with the largest growth rate that dominates the instability. Therefore these numerically sensitive modes can be neglected when considering also the more unstable shear modes. Furthermore, their amplitude tends to decrease with increasing resolution. Within the approximations of the analysis, Figure 5.4a indicates that the Munk layer is unstable to inviscid, growing perturbations.

The phase speed of the fastest growing mode is shown as a function of wavenumber in Figure 5.4b The value varies between a value of $c_{r}=-0.98$ at $l=0.01$ and $c_{r}=-.056$ at $l=6$, both in the range of $\bar{v}$. 
a) U - eigenvectors

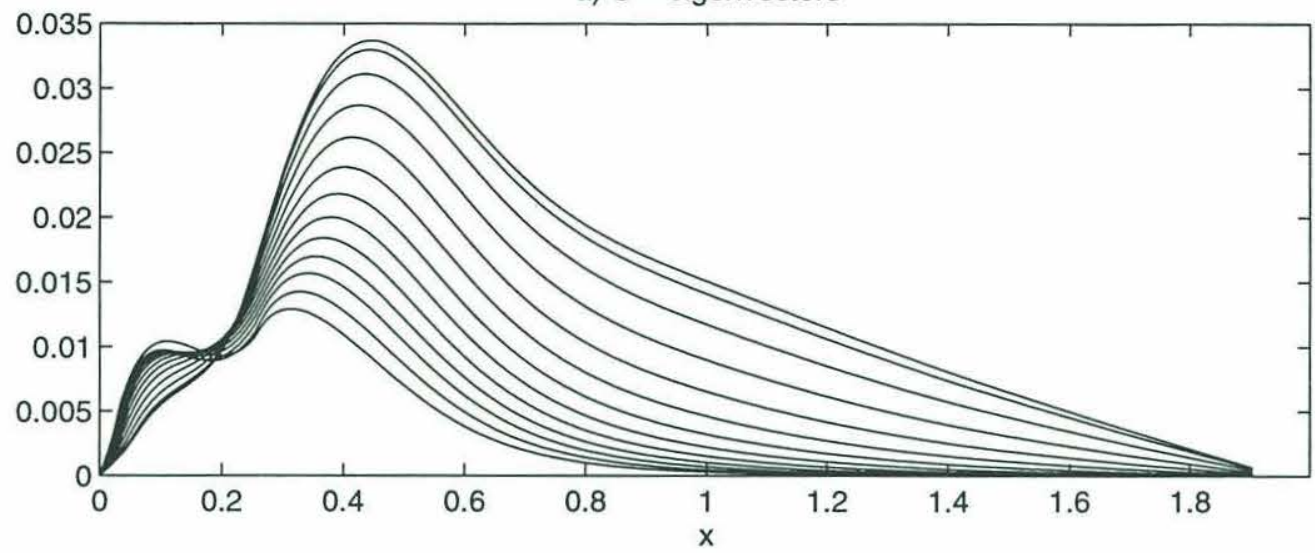

b) V - eigenvectors

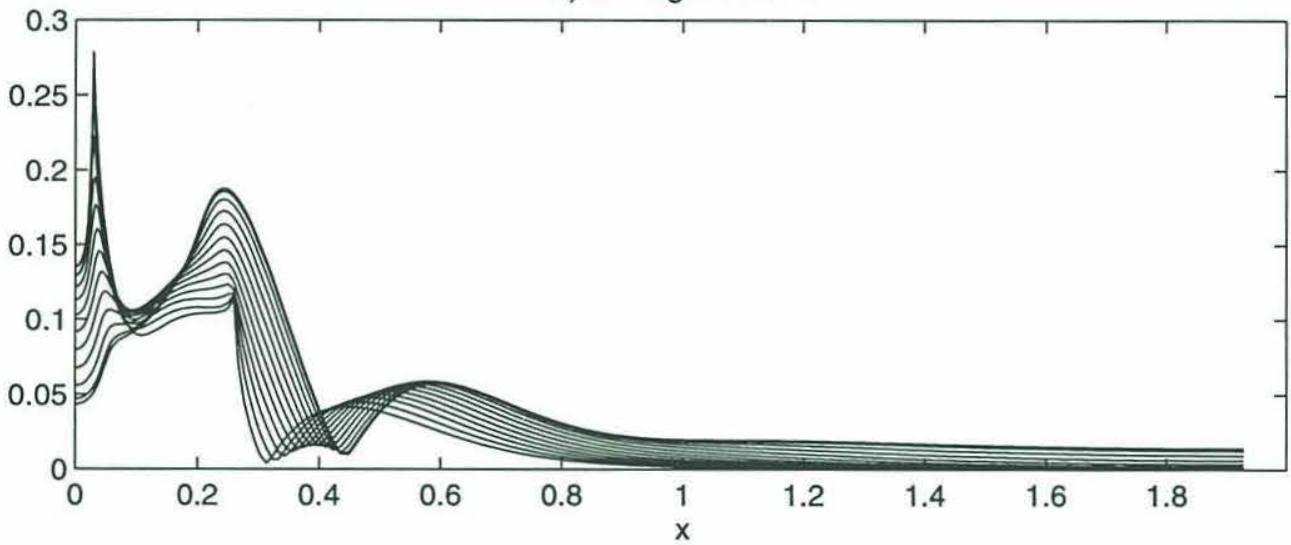

c) $\mathrm{H}$ - eigenvectors

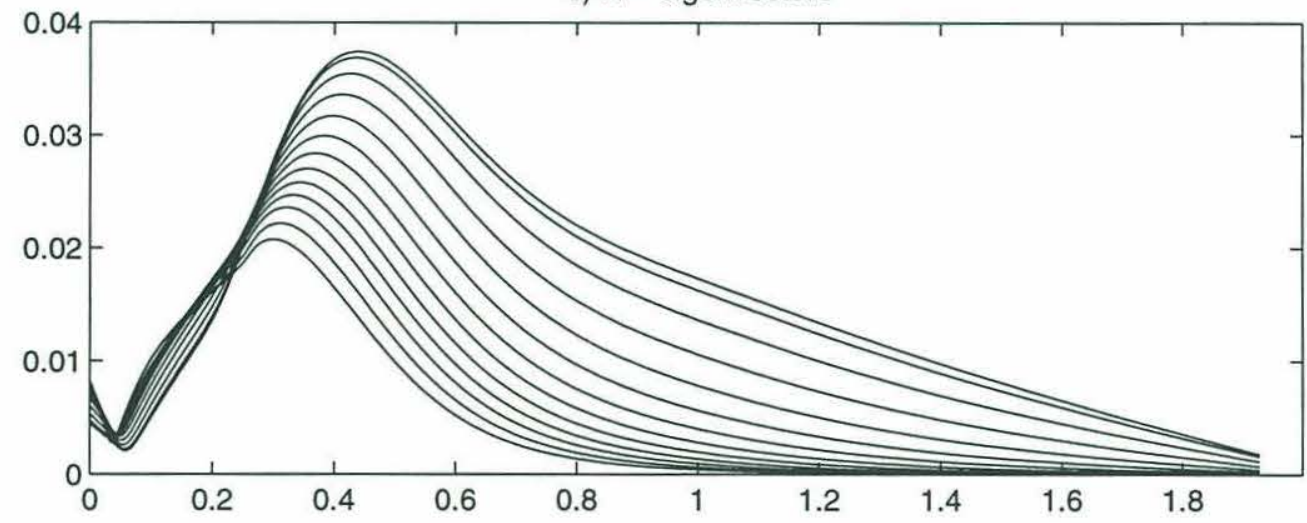

Figure 5.5: Eigenfunction amplitudes of the most unstable modes in Figure 5.4 at intervals of $l=0.5$. (a) U, (b) V, and (c) H. 
The amplitudes of the eigenvectors (e.g., $\sqrt{U U^{\star}}$ ) associated with the fastest growing modes are shown in Figure 5.5. The zonal velocity and height fields are smooth functions of longitude, with a noticeable maximum between $x=0.3$ and $x=0.5$, depending on the wavenumber. At high wavenumbers, the eigenfunctions show the rise of a second peak near $x=0.1$. The $V$ component also has an interesting two peak structure, with a third, particularly sharp feature developing for high wavenumbers. The eigenfunctions are very smooth for long wave solutions. The sharpest peak at high wavenumber is associated with the critical location where Munk velocity profile equals the phase speed of the fastest growing mode,

$$
\bar{v}(x)=c_{r}^{1} .
$$

For a jet profile with the phase speed in the range of the velocity field, this relation is satisfied at least at two longitudes. From Figure 5.4 it is clear that $\bar{v}=0.059$ corresponds to the phase speed of the fastest growing mode at $l=6$. This line is overlayed as a dot-dashed curve in Figure 5.3a. Equality 5.24 applies at the intersection of the two curves. The first intersection occurs at $x=0.275$, which corresponds closely to the peak in the eigenvector amplitude at $x=0.029$. The second intersection occurs at $x=.262$, where the potential vorticity gradient, $\frac{d \bar{q}}{d x}$, is nearly zero. Thus the asymmetry of the Munk boundary layer appears to play a role in the development of the strong peak in the eigenvector curve. It occurs approximately at the location determined by Equation 5.24, but the one which does not satisfy

$$
\frac{d \bar{q}}{d x} \cong 0 .
$$

Identical analyses for a range of latitudes provide the growth rate as a function of wavenumber and latitude. Letting $f=\beta y$ with $\beta=1$ provides the growth rate explicitly as a function of latitude. This field is contoured in Figure 5.6a for the same Munk layer used above. Solid lines separate intervals of a hundredth and two dot dashed contours at values of $\omega_{i}=0.058$ and 0.0584 are used to aid the eye. A growth rate maximum $\left[\omega_{i}\right]_{\max }=0.0584$ resides clearly in the immediate vicinity of the 


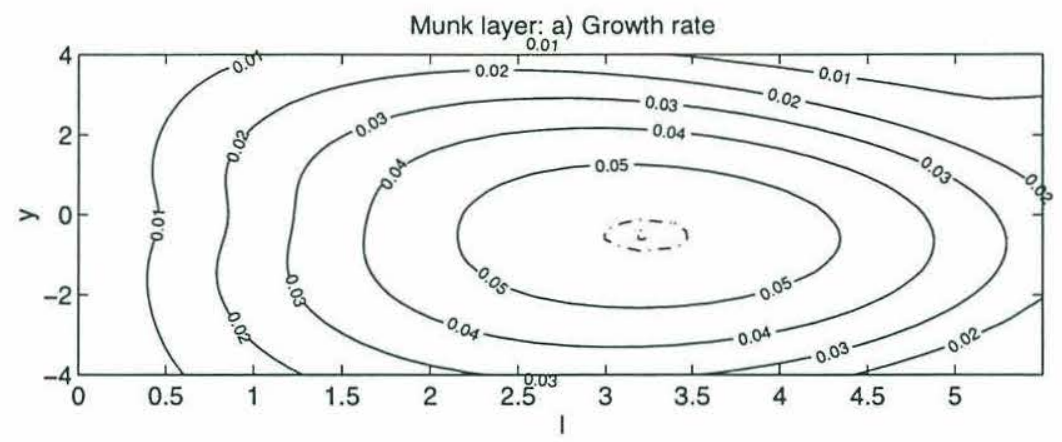

b) Phase speed

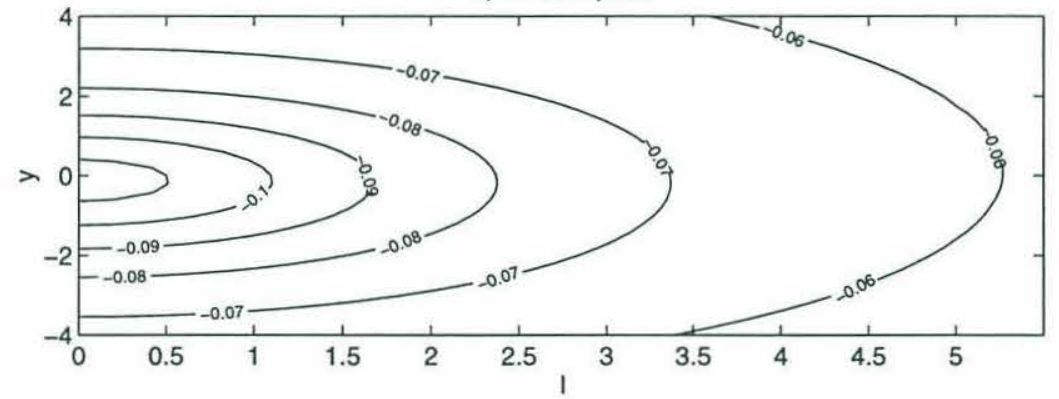

Figure 5.6: As functions of meridional wavenumber and latitude, the a) growth rate and b) phase speed of the fastest growing mode for the Munk boundary layer in Figure 5.3. The dot-dashed curves in (a) mark $\omega_{i}=0.058$ and 0.0584 .

equator, at a wavenumber $l=3.2$ and at a latitude of $y=-0.5$. However, this growth rate maximum is not located at $y=0$. Since the basic state is independent of $y$, it appears that the shallow-water system is aware of the direction of the flow through the absolute vorticity, $f+\bar{v}_{x}$, in matrix, $\hat{\mathbf{A}}$.

The phase speed of the fastest growing mode is contoured in Figure $5.6 \mathrm{~b}$ also as a function of wavenumber and latitude. For a given wavenumber, the phase speed of the most unstable disturbance decreases with latitude. The significance of this structure along with a more physical interpretation of the equatorial enhancement of the instability is further explored in the context of a still simpler model in the next section. 


\subsubsection{Geostrophic Perturbations}

The previous section shows that the Munk boundary layer is unstable to inviscid perturbations, and that the instability is greatest near the equator. However, the complicated form of matrix, $\hat{\mathbf{A}}$, prevents a direct explanation for the tropical enhancement. Two assumptions that follow examples of Stern (1961) and Lipps (1963) significantly simplify the system. First, the mean flow is assumed only weakly nonlinear so that

$$
\bar{v}_{x} \ll f
$$

This approximation is clearly invalid very close to the equator, where $f=0$, but the flow can be specified to be arbitrarily weak so that relationship 5.26 holds for all other latitudes.

Second, the perturbations must be geostrophically balanced,

$$
\begin{aligned}
-f v^{\prime} & =-g^{\prime} h_{x}^{\prime} \\
f u^{\prime} & =-g^{\prime} h^{\prime}{ }_{y} .
\end{aligned}
$$

This relation assumes that the Rossby number of the perturbation is small, which is valid again arbitrarily close to the equator for truly infinitesimal motion. It also assumes the time rate of change of the perturbation be small relative to the Coriolis term,

$$
\frac{l c}{f} \ll 1 .
$$

Since the growth rate of a disturbance in linear theory scales with the velocity, this relation can again be satisfied arbitrarily close to the equator for sufficiently weak flows.

The geostrophic approximation couples the different fields of the disturbance such that a single equation results, expressed in terms of a single variable. Adopting the above approximations and scaling the horizontal dimensions by the length scale, $L$, yields for the height anomaly, $H$,

$$
(\bar{v}-c)\left[H_{x x}-\left(l^{2}+F\right) H\right]-\left(\bar{v}_{x x}-F \bar{v}\right) H=0,
$$


where

$$
\begin{aligned}
F & \equiv \frac{L^{2} f^{2}}{g^{\prime} \bar{h}_{x}} \\
& \cong\left(\frac{L}{L_{D}}\right)^{2}
\end{aligned}
$$

is the rotational Froude number. At the boundaries, the height of the disturbance is set to zero:

$$
H=0 \text {. }
$$

Equation 5.29 describes the initial development of the instability for weak mean flows until the Rossby number of the disturbance, $\frac{l U^{\prime}}{f}$, becomes order 1 . For $F=0$, Equation 5.29 reduces to the familiar Rayleigh equation or the equation for standard barotropic instability. The additional terms proportional to $F$ reflect the influence of the horizontal divergence in the velocity field that is included in the shallow-water model.

Separating the amplitude, $H$, and the phase speed, $c$, into real and imaginary parts and integrating over the domain gives the usual necessary criterion for the development of an instability,

$$
c_{i} \int_{0}^{x_{E}} \frac{G|H|^{2}}{|\bar{v}-c|^{2}} d x=0
$$

where

$$
G=\bar{v}_{x x}-F \bar{v}
$$

is the mass-weighted potential vorticity gradient. Equation 5.32 shows that for growing modes to exist (i.e., if $c_{i}>0$ ), there must be a location within the domain where $G$ changes sign.

The solution to Equation 5.29 subject to boundary conditions 5.31 gives remarkably similar results to that of the full system. The contour plot of growth rate as a function of wavenumber and latitude is shown in Figure 5.7a. Solid contours are uniform, every hundredth, and the dot-dashed contours correspond to values, $\omega_{i}=0.058$ and 0.0584 as in Figure 5.6a. The maximum of 0.0588 is located at a wavenumber of $l=3.26$ 

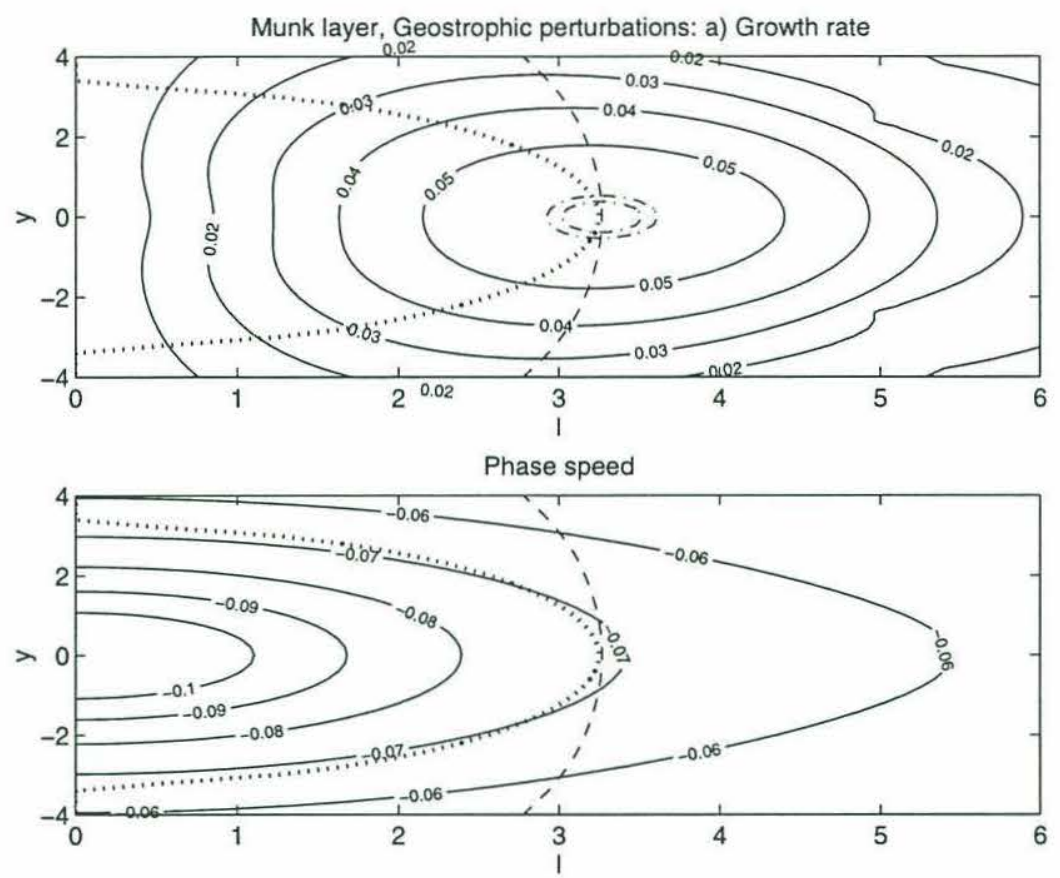

Figure 5.7: As functions of meridional wavenumber and latitude, the a) growth rate and b) phase speed for the fastest growing mode as in Figure 5.6, but assuming geostrophic perturbations as in Equation 5.29. The wavenumber corresponding to the growth rate maximum as a function of latitude is shown as the dashed curve in both plots. The dotted curve is the wavenumber, $l_{m}$, defined in Equation 5.36 as a function of latitude.

and $y=0$. Note that there is no asymmetry in this modified instability problem as Equation 5.29 can not distinguish between the northern and southern hemispheres if the basic state is longitude independent.

The effect of the horizontal divergence of the velocity field can now be interpreted in Equation 5.29 more simply than through the full shallow-water system. Introducing the effective wavenumber, $\kappa$ such that

$$
\kappa^{2} \equiv l^{2}+F,
$$

Equation 5.29 becomes

$$
H_{x x}-\kappa^{2} H-\frac{G}{\bar{v}-c} H=0 .
$$

Consider first the case where $G$ and $\bar{v}$ are independent of latitude. In this case, Equation 5.35 has exactly the form of the Rayleigh equation in terms of the effective wavenumber, $\kappa$. A generic growth rate curve for an unstable mean flow is shown in 


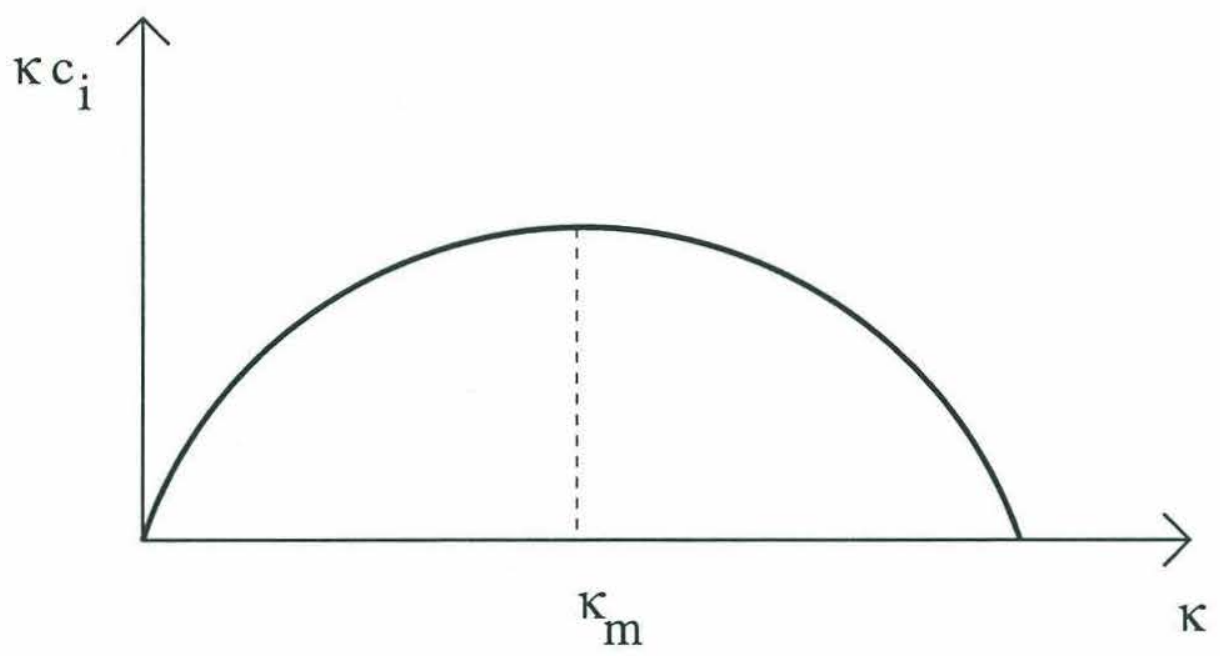

Figure 5.8: Generic growth rate curve for a wavenumber, $\kappa$.

Figure 5.8. The growth rate has a maximum at a value of $\kappa_{m}$ which is independent of latitude. As the Coriolis parameter increases with latitude, the deformation radius $L_{D}$ decreases, and therefore $F$ increases. The actual meridional wavenumber associated with $\kappa_{m}$ is

$$
l_{m}=\sqrt{\kappa_{m}^{2}-F},
$$

which decreases rapidly with latitude. However, the actual growth rate depends on $l$ and not $\kappa$, so the true growth rate maximum does not follow a relation like equation 5.36 , but rather a more modest decrease with latitude. Both $l_{m}$ and the actual growth rate maximum, $l_{M}$, are shown in Figure 5.7 as functions of latitude.

This trend can be expressed alternatively. For a fixed actual wavenumber, $l_{0}$, increasing $F$ with latitude shifts $\kappa$ to a larger value. If $l_{0}<l_{m}$, this effect can induce larger growth rates at that wavenumber, initially. However, this increase ends once $\kappa_{m}$ is reached. Further increases in latitude reduce the growth rate of the instability until ultimately the high wavenumber cutoff, $\kappa_{1}$, is passed, and the flow becomes stable.

Next examine the effect of divergence through the last term on the left hand side of 5.35. From the stability criterion, Equation 5.32, it is clear that if $G$ is of one sign then $c_{i}=0$. If the flow has an inflection point and $F$ is small, then this requirement is satisfied. However, if the flow is of one sign, as for example with a fully inertial 
boundary current, then at some critical latitude, $F\left(y_{c}\right) \bar{v}>\bar{v}_{x x}$ for all $x$, and the flow is stable. In this case, the effect of divergence is also stabilizing. For flows that vanish at least once in the domain, such as the Munk boundary layer, the flow can satisfy the necessary criterion for instability at all latitudes, but the flow may become stable at high latitudes according to the previous argument.

Thus the flow becomes more stable as the ratio of the length scale of the mean flow to the deformation radius increases. A physical interpretation is obtained by considering the instability as the result of interacting vorticity anomalies.

Any curvature in the velocity profile acts as a vorticity gradient upon which Rossby waves propagate. The phase speed of a wave in a meridional mean flow is given in terms of the deformation radius by

$$
c=\bar{v}-\frac{\hat{\beta}}{l^{2}+\left(\frac{L}{L_{D}}\right)^{2}}
$$

where the effective vorticity gradient is

$$
\hat{\beta}=-\bar{v}_{x x} .
$$

A schematic of the western boundary current is presented in Figure 5.9, in which it is clear that the vorticity gradients change sign on either side of the inflection point, $P$. Relative to the velocity, $\bar{v}_{P}$, of the mean flow at $P$, waves travel southward to its east and northward to its west, but are advected by the relative mean flow. Therefore it is possible to have waves that are stationary with respect to $\bar{v}_{P}$. These phaselocked modes interact, advecting anomalous vorticity and encouraging the growth of neighboring disturbances.

As the deformation radius increases, the phase speed of a given wave increases. To maintain stationarity with respect to the mean flow, the wavenumber must decrease. However, decreasing $l$ increases the separation between anomalies, and they interact less strongly. Actual growth is determined by the balance of phase-locking and mutual reinforcement. If only stationarity were important, the wavenumber, $l_{M}$, associated 


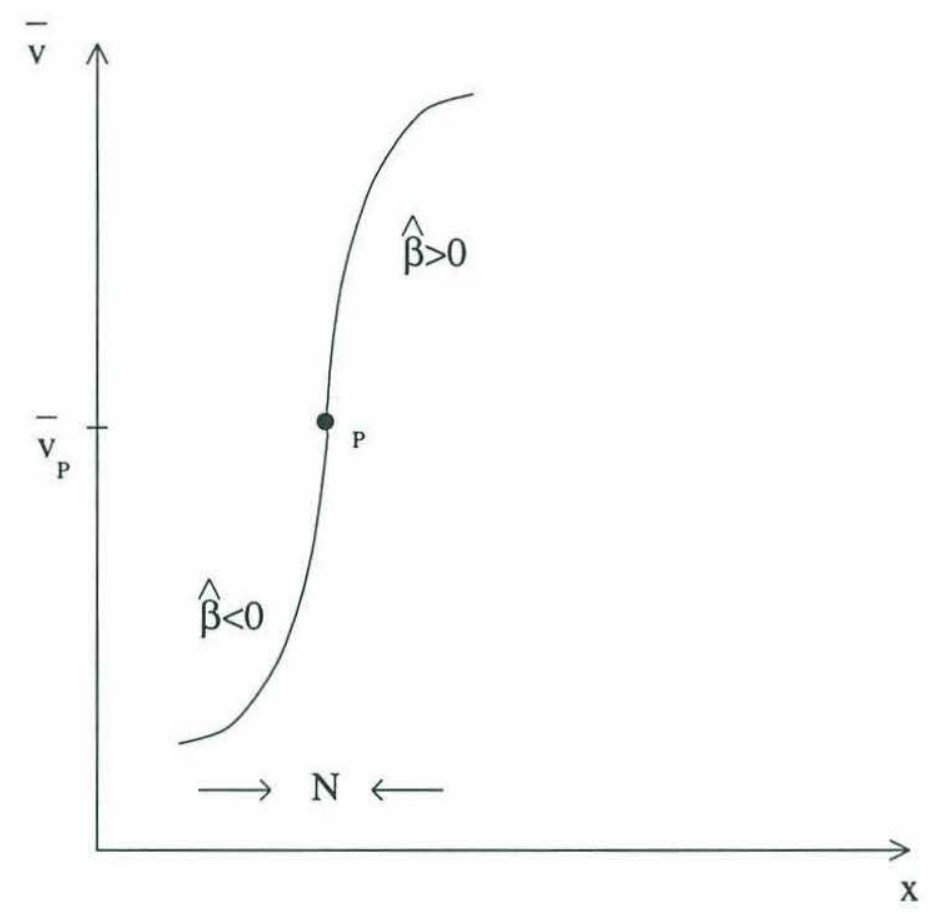

Figure 5.9: A western boundary current profile with an inflection point, $P$. Note that the potential vorticity gradient due to the current changes sign at $P$ which acts as the local "north" pole.

with the maximum in the growth rate would follow the dotted curve in Figure 5.7b which shows $l_{m}$. Note its close association with the lines of constant phase. In fact, $l_{M}$ decreases much less rapidly with latitude to maintain the necessary proximity of anomalies.

The physics of the instability can also be interpreted from the perspective of energetics. In the context of this simplified treatment that considers only geostrophically balanced perturbations, it is possible to derive the energy equation,

$$
\int_{0}^{x_{E}} d x\left\{\frac{\partial}{\partial t}\left[\frac{1}{2}\left(\overline{\left(H_{x}\right)^{2}}+\overline{\left(H_{y}\right)^{2}}\right)+\frac{1}{2} F \overline{H^{2}}\right]=\overline{H_{x} H_{y}} \bar{v}_{x}\right\} .
$$

Here, the overbar symbol with a $y$ superscript, implies an averaging in the $y$-direction. Zonally integrated, the time evolution of total energy of the disturbance depends on the ability of the disturbance to extract energy from the mean flow through the Reynolds stress, ${\overline{H_{x} H_{y}}}^{y}$.

The total energy is divided between potential, the last term on the left hand side of Equation 5.39, and kinetic, the first two terms, and their ratio is $\mathrm{O}(F)$. At mid- 
latitudes, $F$ is large, and most of the energy of the disturbance is partitioned into potential form $(P)$. In contrast, near the equator the fraction of total energy that is kinetic $(K)$ reaches its maximum. However, from the right hand side of Equation 5.39, it is clear that the growth of the instability is proportional to the Reynolds stress, and the Schwarz inequality allows

$$
\left(H_{x} H_{y}\right) \leq \frac{\left(H_{x}\right)^{2}+\left(H_{y}\right)^{2}}{2} .
$$

Seeking solutions,

$$
H=\hat{H}(x, y) e^{\sigma t},
$$

it can be shown that

$$
\int d x\left\{\sigma(K+P) \leq K\left(\bar{v}_{x}\right)_{\max }\right\},
$$

or

$$
\int d x\left\{\sigma \leq \frac{K}{K+P}\left(\bar{v}_{x}\right)_{\max }\right\} .
$$

Thus the growth rate of the disturbance depends on the ratio of the kinetic energy to the total energy. When the partitioning favors potential energy, this ratio is small and the disturbance grows more slowly than when more of the energy is in kinetic form. At the equator, where the system is most barotropic and the kinetic energy dominates, the disturbance extracts energy from the mean flow most efficiently. At mid-latitudes, where much of the energy is stored in layer height variations, the Reynolds stress is small, and the instability is more subdued.

It is important to note that the original work of Lipps (1963) illustrated the stabilizing effect of divergence on a zonal mid-latitude flow, and this work was extended to the equator by Philander (1976).

\subsubsection{Instability of an Inertio-Viscous Current}

The analysis above reveals that the Munk boundary current is inviscidly unstable. However, western boundary currents found in nature and in realistic numerical models 
likely do not satisfy a linear vorticity balance. To examine the stability of a more realistic mean state, the output of the time-dependent numerical model is used to provide a basic state. The model output is shown in Figure 5.2a, which is spun up just below threshold with $R e=25$. Note that this profile technically violates the parallel flow assumption, 5.4, because it varies slightly with latitude. However, the variations with longitude are much larger than those in $y$, and this detail is ignored. Velocity and height profiles are taken directly from the numerical model output and interpolated onto the relevant numerical grid for the instability calculations using a cubic spline interpolant. This section applies the full, inviscid shallow-water formulation using $\hat{A}$ as opposed to the simplified model of the last section.

Figure 5.10a presents the two-dimensional plot of growth rate versus latitude and meridional wavenumber. The highest wavenumber data have been cropped to omit noise that appears there from the numerical modes that slightly dominate there. Comparison with Figure 5.6 show that the two profiles have distinctly similar instability characteristics, despite the addition of a mildly latitude-dependent basic state and a slightly more nonlinear profile. The maximum growth rate, $\left[\omega_{i}\right]_{\max }=0.0574$, is slightly less than that for the Munk profile, and it occurs at $l=3.2$ and $y=-.5$ identical to the other calculation. The phase contours in Figure $5.10 \mathrm{~b}$ are also very similar to the Munk curves, though slightly shifted in magnitude apparently by slight differences in profile.

It is surprising that the behavior of the latitude-independent and latitude-varying profiles are so similar. The potential vorticity gradient of a single layer model takes the form

$$
\frac{d \bar{q}}{d x}=\frac{\bar{v}_{x x}-\frac{f^{2}}{\bar{h}} \bar{v}}{\bar{h}} .
$$

If the height field is in geostrophic balance with the velocity, then the denominator in the rightmost term above might distinguish the stability properties of one profile versus the other and induce its own hemispheric asymmetry. However, the term is multiplied by $f^{2}$, so its effect is small near the equator where the dynamics are dominated by the 


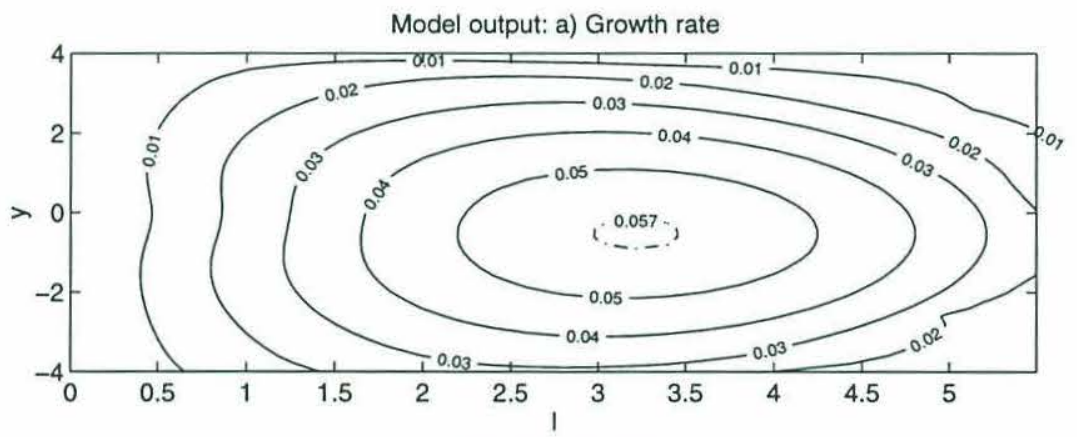

b) Phase speed

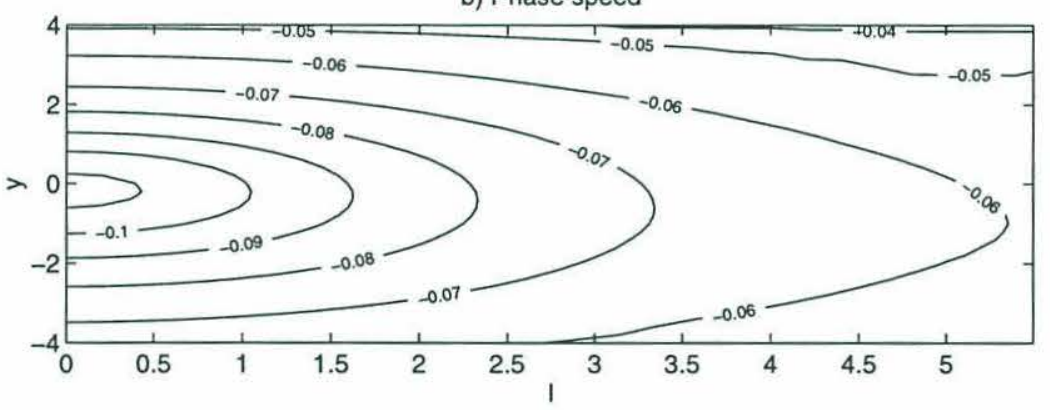

Figure 5.10: As functions of meridional wavenumber and latitude, the a) growth rate and b) phase speed of the fastest growing mode as in Figure 5.6 except that the basic state used is from the numerical model experiment shown in Figure 5.2a. The dot-dashed curve in (a) demarcates $\omega_{i}=0.057$. 
first term. Thus the instability properties are quite robust to meridional variations in the basic state.

\subsection{The Influence of Viscosity}

The previous calculations applied to inviscid perturbations. However, viscosity is an essential part of the dynamics of the mean flow, and therefore it should be expected to influence the dynamics of the perturbation. Indeed the fact that the system is stable for some Reynolds numbers shows that its effect is not negligible. The eigenvalue problem including dissipation along with the extra, no-slip boundary condition is solved similarly to the above inviscid problems. The stability of the basic state is examined for a range of viscosities.

The eigenvalues for numerical model output data at $y=1$ determined using a viscosity, $A_{H}=2.5 \times 10^{-4}(R e=100)$ are shown in Figure 5.11. One feature that distinguishes these results from those in Figure 5.4 is that the eigenvalues no longer come in complex conjugate pairs. The introduction of viscosity changes the symmetry of the problem, and causes most modes to decay in time. In fact, only those modes near the imaginary axis are shown in the Figure so as to illustrate the more interesting single set of growing modes. These correspond directly to those fastest growing modes in the inviscid problem, now slightly damped by the introduction of a small amount of friction, but not sufficiently to damp the instability. One change is that the maximum in the growth rate curve is shifted out to higher wavenumber, $l=4$. In addition, the marginal points that bound the region of stability from instability are shifted somewhat from the inviscid problem. The phase speeds associated with that set are shown in Figure 5.11b, and have a similar character to those of the inviscid problem.

The amplitudes of the eigenfunctions of the unstable modes are shown in Figure 5.12 and again, their similarity to the inviscid case is also striking. One disti nguishing feature of the viscous modes is found in the amplitudes of the $V$ eigenfunctions. As 

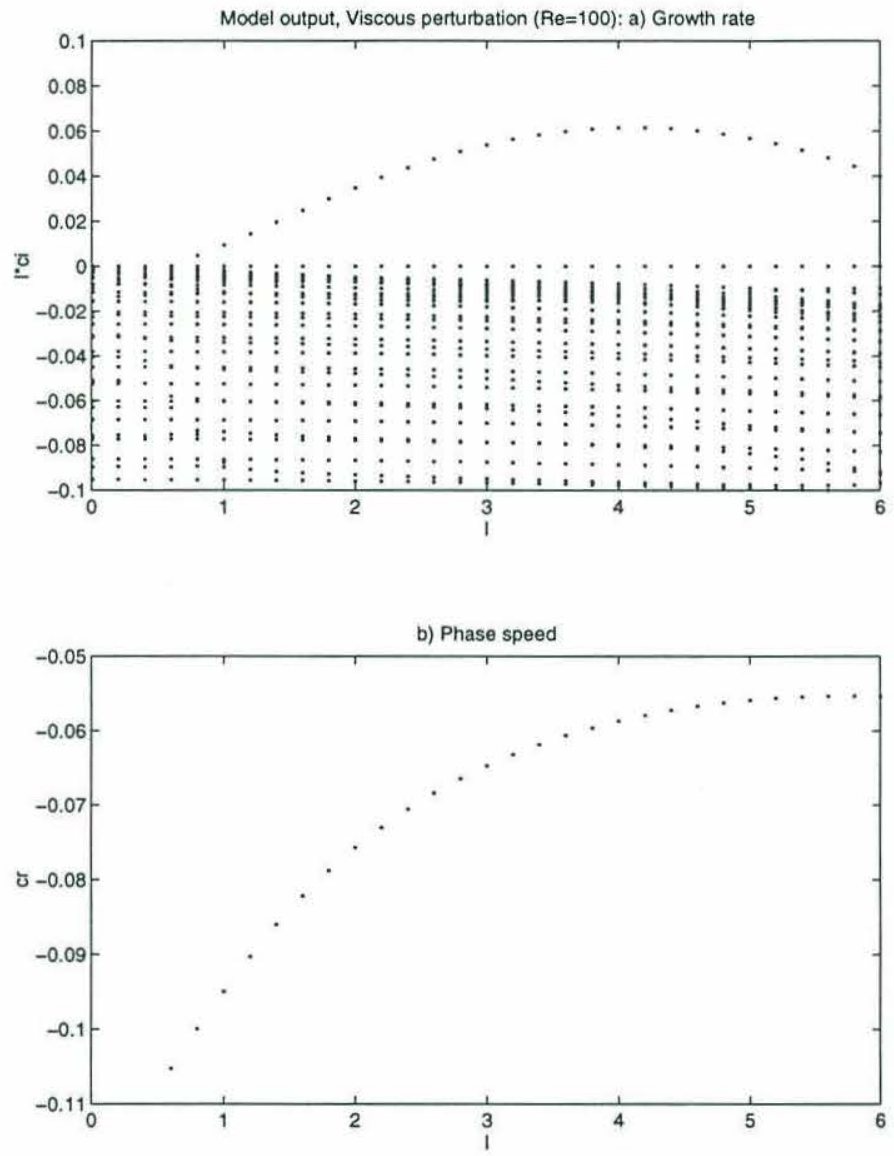

Figure 5.11: As functions of meridional wavenumber, the a) growth rate and b) phase speed of the fastest growing mode for the viscous stability problem using the model output profile. Calculations assume $R e=100$ to compare directly with the inviscid calculations of the Munk boundary layer. 
in the previous problem, a peak appears at small $x$ in the functions corresponding to large wavenumber. However, the peak is broader than in the inviscid case suggesting that viscosity plays an important role in the dynamics of the critical layer.

These calculations apply a large Reynolds number explicitly to compare directly the inviscid calculations of the Munk boundary layer to an approximately inviscid calculation with a small amount of viscosity. The close agreement between modes and eigenfunctions suggests strongly that the basic mechanism of the instability is identical to the inviscid problem, namely shear instability.

Calculations at several latitudes show similar bullseye patterns of growth enhanced near the equator, but with reduced amplitude depending on the magnitude of the viscosity employed. For example, using a viscosity of $8.333 \times 10^{-4}$ which corresponds to $R e=30$ gives a maximum growth rate, $\left[\omega_{i}\right]_{\max }=0.014$, centered at $l=3.8$ and $y=-1.2$. Increasing the dissipation appears to drive the instability further south of the equator, and lengthen the time needed for its development. The shift to a higher wavenumber is found consistently at all latitudes.

This highly idealized viscous theory indicates that the numerical model should be unstable for $R e=25$. In fact, numerical integrations indicate that the instability begins near $R e=32$. This small discrepancy may result from the approximations of the instability calculation itself (e.g., neglect of the variation in $f$ ). The numerical model may in fact be unstable for $R e=25$. The time-scale for growth of the instability at this Reynolds number is $\mathrm{O}(5000)$ time units, which is a very long integration that I have not examined. At $R e=32$ the time-scale is $\mathrm{O}(300)$ time units, which is the time-scale over which they are observed to develop. Thus the numerical model may be unstable at smaller Reynolds numbers than $R e=32$, but so slightly that the time-dependent motion escapes detection.

A second measure of the accuracy of the instability calculation is to compare the length scales of the disturbance predicted by the theory to that found in the numerical experiment. After reaching an approximate steady state at $R e=31.25$, the viscosity 


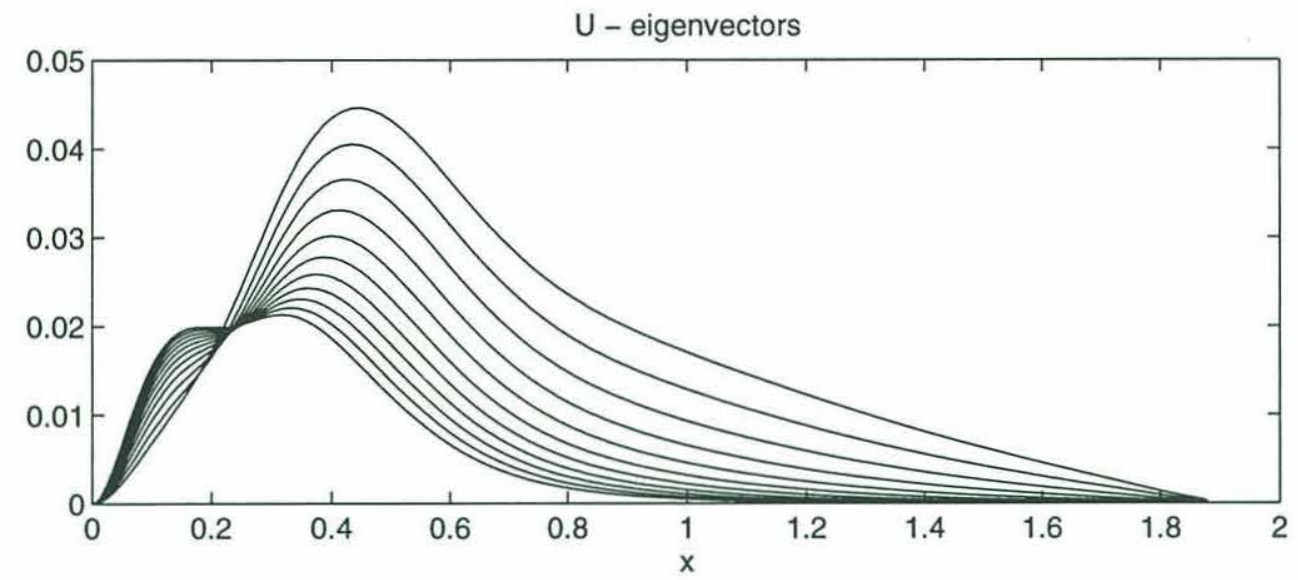

$\mathrm{V}$ - eigenvectors

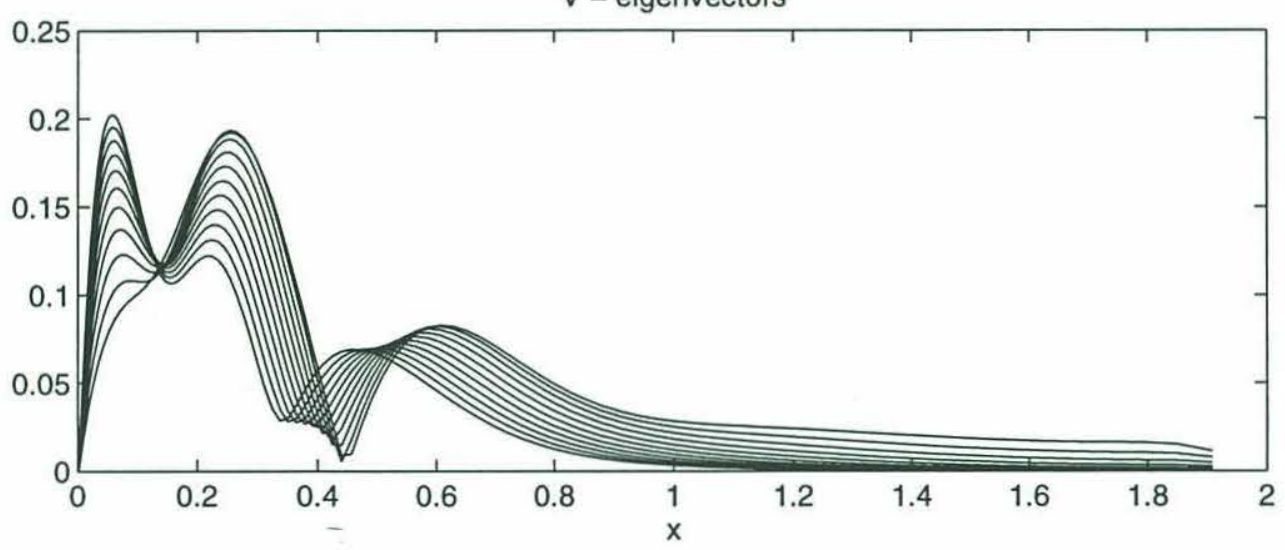

$\mathrm{H}$ - eigenvectors

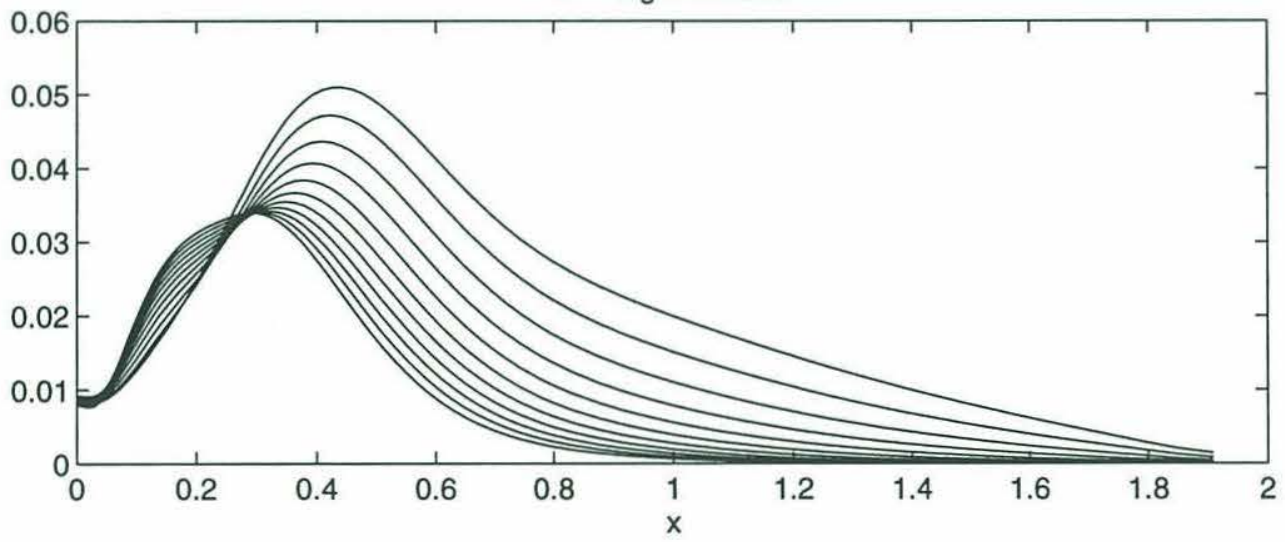

Figure 5.12: Eigenfunction amplitudes of a subset, $l=1$ to $l=6$ in intervals of 0.5 , of the fastest growing modes in Figure 5.11: (a) U; (b) V; (c) H. Notice the broad peak in the $V$ eigenfunction near $x=0.05$ 


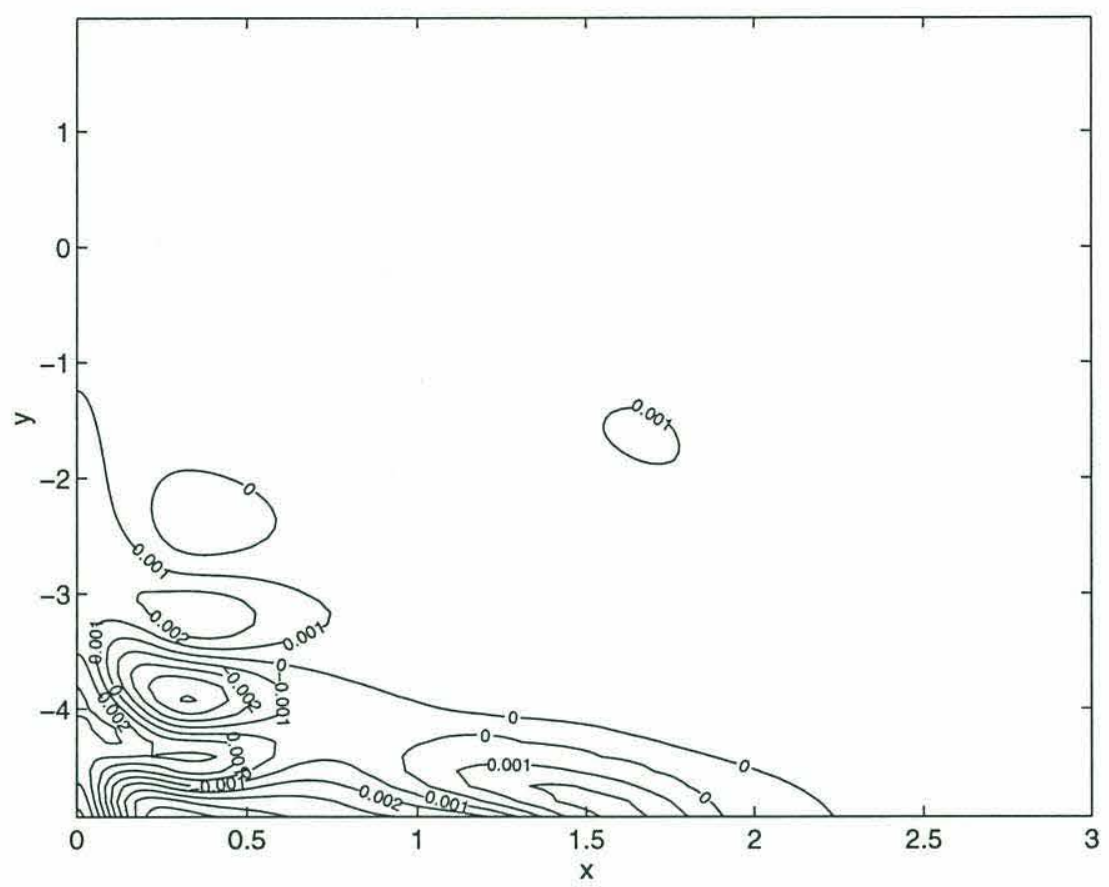

Figure 5.13: The height field anomaly after instability begins. The difference in height fields between steady solution at $R e=31.25$ to $R e=32.25$ after 300 time units.

of the numerical model was changed slightly to $R e=32.25$, and integrated further in time. Figure 5.13 presents the difference in the height fields after 300 time units have elapsed. Height anomalies appear just south of the equator as expected from the theory, and develop further to the south. There is some variability being swept eastward from the western boundary near the southern boundary of the plot, where the boundary current veers eastward as part of the southern hemisphere sink. Approximately one full wave is visible in the figure, and it has a wavelength of approximately 1.5 deformation radii. This wavelength agrees well with that predicted by the theory of $\lambda \cong \frac{2 \pi}{4}$. Thus even with the extensive idealizations of the theory, it predicts the behavior of the disturbances found in this numerical model, in location for growth, in wavelength, and in the critical Reynolds number.

The numerical example described above contains eddies that are approximately of the deformation scale, consistent with the linear theory and the basic state applied. To demonstrate that the eddy dimension is in fact determined by the width of the 

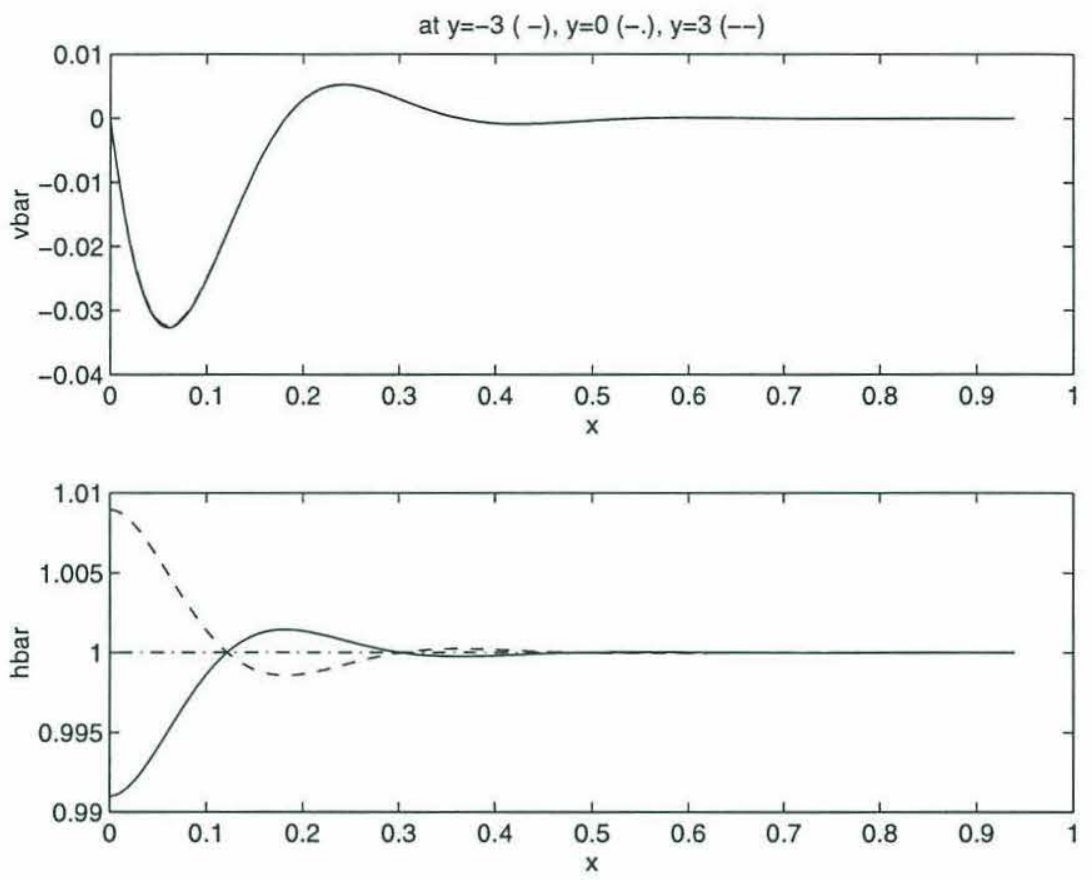

Figure 5.14: a) Velocity and b) height fields as a function of $x$ at three latitudes in the basin $(y=3(-), y=0(-$.$) , and y=-3(-))$. This profile for a second experiment has a zonal scale that is roughly half that shown in Figure 5.2a.

boundary current, and not the deformation radius, I have examined a second numerical experiment in which the viscosity has been reduced by a factor of eight. Setting $A_{H}=1.25 \times 10^{-4}$, a steady solution results from using $H=1$ and $S_{0}=0.003$. For these parameters, $R e=24$ is just below the critical value at which time-dependent motion begins, which indicates that $R e_{c}$ is not a universal number, but varies with model parameters, such as $\frac{\delta_{M}}{L_{D}}$.

Inserting the numerical solution at time $t_{0}=1000$ (shown at three latitudes in Figure 5.14) into the viscous stability model as its basic state gives growth rates as a function of the viscosity that affects the perturbation. For a Reynolds number of 24 , the growth rate is nearly zero. Choosing a value for the viscosity, $A_{H}=1.071 \times$ $10^{-4}$, reveals that the boundary current is unstable to infinitesimal perturbations at $R e=28$. The growth rates and phase speeds for this experiment are shown in Figure 5.15 as functions of meridional wavenumber and latitude. Note that the growth rate maximum occurs at a meridional wavenumber of $l=7.6$, approximately 

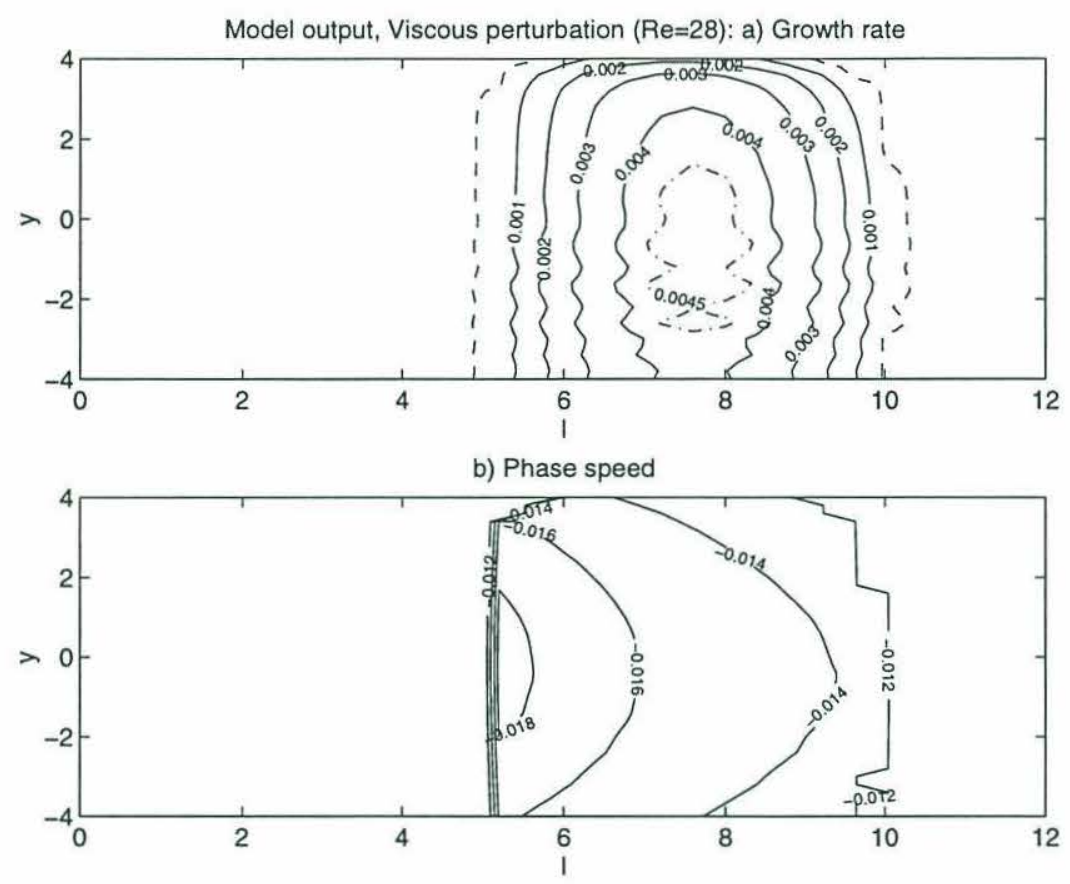

Figure 5.15: As functions of meridional wavenumber and latitude, a) growth rates and b) phase speeds of the most unstable modes for the basic state shown in Figure 5.14, using a viscosity of $A_{H}=1.071 \times 10^{-4}$ ( $R e=28$ for the perturbations). The dashed contour corresponds to a value of $\omega_{i}=10^{-4} \beta L_{D}$ and divides the domain into regions of growth and regions of stability.

twice the value in the former experiment (see again Figure 5.11) and consistent with the smaller boundary current here. The bullseye for this run is also somewhat more extended meridionally from the previous examples, showing that as the boundary current narrows with respect to the deformation radius and the system becomes more barotropic, the tropical enhancement to the instability is less pronounced.

To compare the growth rates predicted by the theory to those observed in the model, I carry out further integration of this numerical experiment at the lower viscosity $\left(A_{H}=1.071 \times 10^{-4}\right)$. This exercise generates cyclonic structures in the vicinity and south of the equator much as in Figure 5.2b, but of a smaller scale. Showing the zonal velocity anomaly, $d u=u\left(t_{0}+\Delta t\right)-u\left(t_{0}\right)$, at intervals of $\Delta t=400$, Figure 5.16 presents the time-evolution of the instability. The contour interval is the same in each plot $(d u=0.002 c)$. After the first time interval, the western boundary current has superposed on it a series of very weak structures of alternating sign. After $\Delta t=800$ 
$\left(\beta L_{D}\right)^{-1}$, well-defined eddies of alternating sign emerge between $y=-1$ and $y=-5$. As time continues, the variability grows, in strength and in meridional extent. After 1600 time units, the eddies exist north of the equator, and the strength of those near $y=-3$ have peaked in strength.

Although the negative anomalies (dashed in the figure) tend to be slightly larger in magnitude than the positive, there is very good symmetry between positive and negative features. This remains true for the meridional velocity and height fields, though the vorticity field shows a more pronounced asymmetry favoring positive anomalies. This symmetry supports the modal decomposition of the $u, v$, and $h$ fields in Equations $5.9 \mathrm{a}$ and $5.9 \mathrm{~b}$. The time-scale for the eddy development in the model is 800 to $1000\left(\beta L_{D}\right)^{-1}$, slightly less than the value predicted by the viscous theory (1300 $\left.\left(\beta L_{D}\right)^{-1}\right)$, and somewhat longer than that predicted by the inviscid calculation (250 $\left(\beta L_{D}\right)^{-1}$, not shown). The fully developed anomalies in Figure 5.16d have a characteristic wavelength of between $\frac{2}{3}$ and 1 deformation radius, depending on the latitude at which the estimate is made. The value predicted by the stability analysis is $\lambda=\frac{2 \pi}{7.6} \cong 0.8$. The inviscid calculation predicts a wavelength of approximately 1 deformation radius. The theory reasonably predicts the time and space scales of the eddies, their location for development, and the critical Reynolds number at which they appear.

The addition of viscosity to the system has adjusted the instability properties slightly, shifting the wavenumber at which the maximum growth rate is found and reducing the amplitude of the growth. However, it has not produced a qualitative change in the development of the system. Viscosity acts as a stabilizing influence, but the physics of the instability is unaltered. These features suggest that the instability mechanism responsible for the growth of the eddies in the time-dependent model is classical shear instability and not viscous instability. 


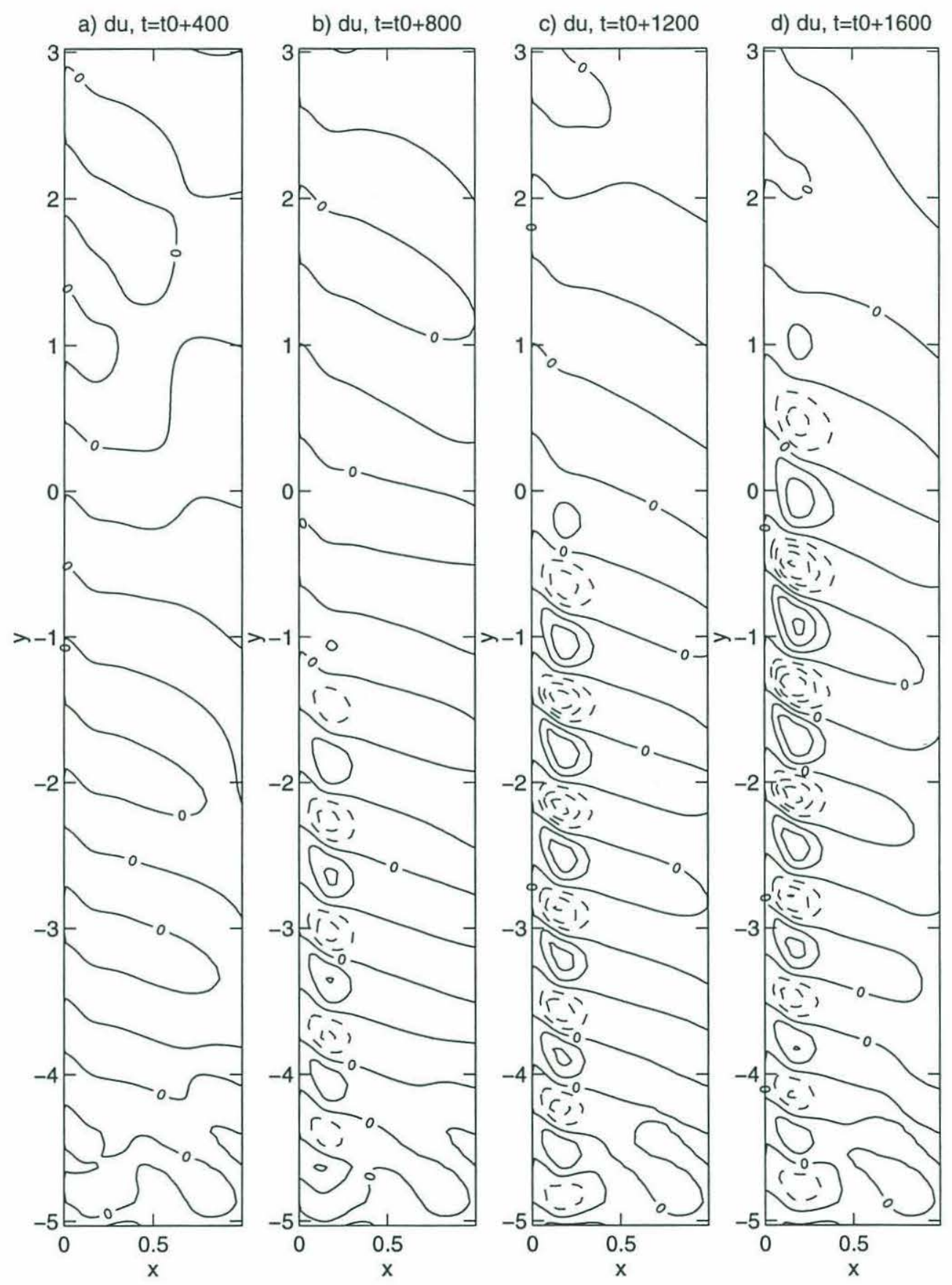

Figure 5.16: Zonal velocity anomalies at intervals of $\Delta t=400\left(\beta L_{D}\right)^{-1}$ showing the development of the eddy field after the time, $t_{0}=1000$, when the Reynolds number of the system was adjusted from 24 to 28 . 


\subsection{Inertial Instability}

Before concluding that shear instability is solely responsible for the development of the eddies, it is worthwhile to examine briefly the possibility of inertial instability. As mentioned in the introduction to this chapter, this instability arises in geophysical flows when the local absolute vorticity is of the opposite sign to the local planetary vorticity, as is likely to occur in cross-equatorial flow. This section derives the necessary stability criterion for inertial instability in the shallow water system, but shows that it is a not likely to be acting on the western boundary current of the model.

To derive the instability criterion, return again to the inviscid system, described by Equation 5.16. In order to isolate inertial instability from shear instability it is helpful to examine a basic flow structure that has no potential vorticity extremum. The analysis in this section thus considers flows that differ substantially from the western boundary currents discussed above to illustrate the basic mechanism of inertial instability. In particular, I examine the stability of parabolic flows which vary very slowly in longitude.

The WKB expansion (e.g., Bender and Orszag (1978)) begins with the introduction of a small parameter, $\epsilon$, which defines a slow variable, $X=\epsilon x$, over which changes in the character of the eigenfunction solution occurs. Eigenfunctions are expressed in terms of $x$ and $X$ as

$$
U(x)=\hat{U}(X) e^{\frac{i k(x)}{e}}
$$

and similarly in $V$ and $H$. The phase, $k$, is also assumed a slowly varying function and can be expressed as a series in powers of the small parameter, $\epsilon$ :

$$
k(x)=\sum_{n=1}^{\infty} \epsilon^{n} k_{n}(x) .
$$

The amplitude and phase of the perturbation can vary on the scale $x$, but the rate of change of the phase varies slowly. Then

$$
U_{x}=\frac{i}{\epsilon} \sum_{n=1}^{\infty} \epsilon^{n} \frac{d k_{n}}{d x} U+\epsilon \hat{U}_{x} e^{\frac{k x}{\epsilon}}
$$




$$
=i \frac{d k_{1}}{d x} U+O(\epsilon)
$$

To leading order, Equation 5.16 reduces to

$$
\left[\begin{array}{ccc}
\bar{v}-c & \frac{f}{l^{2}} & \frac{i}{l^{2}} \frac{d k_{1}}{d x} \\
f+\bar{v}_{x} & \bar{v}-c & 1 \\
\bar{h}_{x}+\bar{h} i \frac{d k_{1}}{d x} & \bar{h} & \bar{v}-c
\end{array}\right]\left[\begin{array}{c}
U \\
V \\
H
\end{array}\right]=0 .
$$

A non-trivial solution requires that the matrix in Equation 5.48 have a non-zero determinant. Then,

$$
(\bar{v}-c)\left[(\bar{v}-c)^{2}-\bar{h}+\frac{f\left(f+\bar{v}_{x}\right)}{l^{2}}-\frac{i}{l^{2}} \frac{d k_{1}}{d x}\left(\bar{h}_{x}+i \frac{d k_{1}}{d x} \bar{h}\right)\right]+\frac{1}{l^{2}}\left(f \bar{h}_{x}+\bar{v}_{x} i \frac{d k_{1}}{d x} \bar{h}\right)=0,
$$

which gives the general stability properties for the shallow-water system under the assumptions of the WKB approximation (i.e., that the rate of change of the phase of the disturbance is small).

The conditions that imply $c$ complex are not obvious from the form of Equation 5.49. As a result, I consider the limit of $\frac{d k_{1}}{d x} \rightarrow 0$, which requires that the amplitudes of the eigenfunctions vary according to the slow variable, $X$. Physically this assumption should correspond to the disturbance of a very slowly varying mean field. If the disturbance senses a homogeneous basic state, then the eigenfunction must be approximately independent of location. Assuming also that the mean height and velocity fields are geostrophically balanced, and examining only that particular location where $\bar{v}=0$, then three eigenvalues are derived,

$$
\begin{aligned}
c & =0 \\
c & = \pm \sqrt{\bar{h}+\frac{f\left(f+\bar{v}_{x}\right)}{l^{2}}} .
\end{aligned}
$$

The associated frequencies are

$$
\omega=0, \pm \sqrt{l^{2} \bar{h}+f\left(f+\bar{v}_{x}\right)} .
$$

For any $f\left(f+\bar{v}_{x}\right)<0$, there is some wavenumber for which the radical in Equation 5.51 is negative, and $\omega$ is complex. The maximum growth rate occurs for $l=0$, which 
distinguishes this process from shear instability whose maximum occurs for non-zero $l$. Thus inertial instability in the two-layer fluid generates long-wave disturbances.

Requiring $\omega$ real in Equation 5.51 gives

$$
f\left(f+\bar{v}_{x}\right) \geq 0
$$

which is exactly the stability criterion derived by Ripa (1983) for a zonal flow on the $\beta$-plane assuming purely longitude-independent motions ( $l=0$ in this case).

Relation 5.52 is independent of conditions 5.17 and 5.18 and simply provides the necessary condition that ensures inertial stability. Absolute stability is guaranteed by the criteria 5.17 and 5.18. A flow may be found to violate the condition for inertial instability, 5.52 , but satisfy the conditions for the shallow-water system, and therefore be stable.

To see whether the criterion 5.52 has any practical use, I have applied the inviscid stability equation, 5.16 , to a flow that is expected to be inertially unstable. The mean flow has a parabolic profile of amplitude $\mathrm{P}$,

$$
\bar{v}=P\left(1-\gamma \frac{\left(x-x_{0}\right)^{2}}{L^{2}}\right),
$$

where $x_{0}=\frac{L}{2}$ is the center of the profile in the center of the channel of width, $L$, and

$$
\gamma=4
$$

ensures the velocity goes to zero at the boundaries. Without an inflection point, this profile isolates the experiment from shear instability. The basic state height field is in geostrophic balance with the velocity.

Setting $P=2$ violates criterion 5.18 and using $f_{0}=0.01$ ensures that condition 5.52 is not satisfied. Calculations from this flow, with no stretching and $N=100$, show no evidence of a long-wave instability. Similar runs, with different parameters appear to be stable also. In fact, I have been unable to find any instability in a parabolic profile on an $f$-plane except for those in which the potential vorticity gradient changes 
sign, which can occur if relation 5.54 is relaxed, and the velocity is allowed to change sign. 1

While the stability criteria $5.17,5.18$ and 5.52 are suggestive, they provide no insurance that the flows are in fact unstable. Inertial instability appears to be very fragile, depending sensitively on the details of the jet. Returning to the subject of the instability of the western boundary current, it appears unlikely that inertial instability is responsible for the development of the eddies. Not only is the physical mechanism sensitive, but it also predicts long-wave features, in contrast to the roughly deformation radius scale eddies that develop in the model.

\subsection{Summary and Discussion}

Linear stability analyses of parallel flow in the $f$-plane shallow-water system show that the western boundary current is unstable to infinitesimal perturbations, and that this instability is greatest in the vicinity of the equator. Three possible driving mechanisms are shear, viscous, and inertial instability. Inviscid shear instability calculations agree well with numerical model results, predicting both the correct length scale for the disturbances and the latitude where they develop. Viscosity, when added to the theory, acts primarily to damp the growth of the instability and modifies the eigenfunctions of the growing modes only quantitatively, and in the vicinity of a critical layer. These facts lead me to conclude that viscous instability is not the dominant process. The viscous calculations do predict the critical Reynolds number at which the western boundary current becomes unstable in reasonable agreement with the timedependent experiments. An analytic treatment of the shallow-water system suggests that it supports inertial instability under very restrictive conditions, but numerical calculations of realistic flow structures (though not resembling the western boundary

\footnotetext{
${ }^{1}$ Interestingly, the parabolic profile with vanishing boundary velocity does appear to be unstable also for a zonal, easterly jet on the $\beta$-plane centered on the equator. This profile is also distinguished in that the meridional potential vorticity gradient changes sign.
} 
current), show that this type of instability is not robust. It seems unlikely that this process plays an important role in the instability of the western boundary current.

These results are consistent with mid-latitude model results of Ierely and Young (1991). That study includes the meridional variation of the Coriolis parameter, but they also examine the case of $\beta=0$. However, while they determine that the central process is viscous instability, I conclude that the boundary current suffers a shear instability. This statement implies that the results of this study are more generally applicable. Any boundary current whose velocity scale decreases or simply levels off to a constant value near the boundary, such as the mean velocity in Figure 1.3, satisfies this criterion and therefore is likely unstable provided a sufficiently high Reynolds number.

Consistent with numerical model results, the tropics exhibit preferred eddy growth in both the viscous and inviscid calculations. The latitude dependence results from the increase of the internal deformation radius with decreasing latitude which modifies the phase speeds of the waves that travel on the vorticity gradient of the mean field. It is ironic that it is the baroclinic nature of the shallow-water model that allows the equator to distinguish itself from all other latitudes, and yet it is the fact the local dynamics at the equator are most barotropic that allows for the enhanced instability there.

Several approximations, such as the neglect of the meridional variation in the planetary vorticity and mean flow, have aided the above analysis and contributed to the simple physical interpretation. It is possible in the context of the simple model of Section 5.3.3 to relax one simplification and consider the effect of $\beta$. Carrying out the same analysis with the vorticity equation and maintaining the divergence in the velocity field yields a similar eigenvalue relation to Equation 5.11. Calculations based on this model show similar features to those in the $f$-plane model, with an equatorially enhanced growth rate, and comparable phase speeds of the unstable modes (Figure 5.17). Interestingly, the growth rate maximum is somewhat larger than without $\beta$ (compare $\left[\omega_{i}\right]_{\max }=0.085$ to 0.058 ). As in the study of Ierley and Young (1991), 

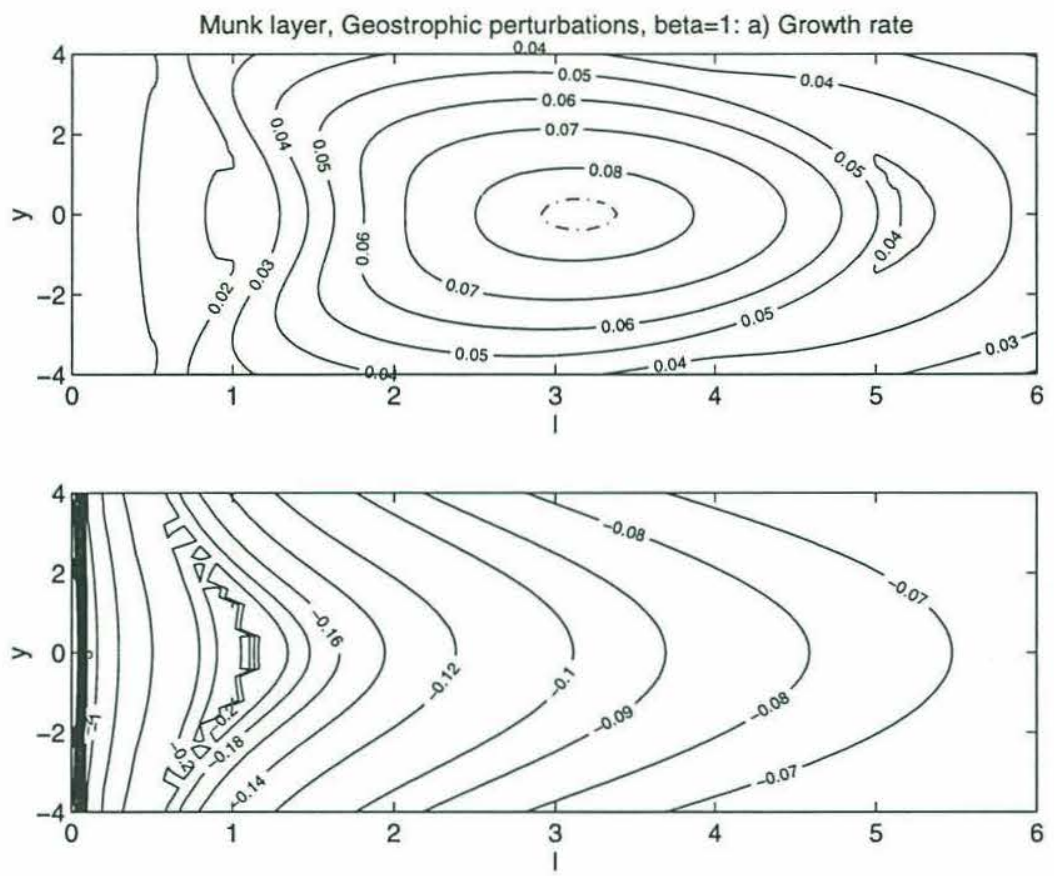

Figure 5.17: As functions of meridional wavenumber and latitude, the a) growth rate and b) phase speed as in Figure 5.7 except that $\beta=1$ is included.

basin modes develop at the small wavenumbers, a result of the meridional vorticity gradient, but these have small growth rates compared to the modes that decay with $x$.

The effect of a vorticity gradient raises interesting possibilities when considering more realistic oceanic applications. For example, western boundary currents are seldom found along purely meridional, perfectly vertical walls. Borrowing the results from studies of barotropic instability in zonal flows suggests that a topographic $\beta$, which is likely to be relevant for deep boundary currents, stabilizes the instability at mid-latitudes but not at the equator. Thus topographic influences would further enhance the tropical nature of the instability. Also, the effect of a sloping coast breaks the symmetry of the instability process, with westward currents being more unstable.

The agreement between the simplified analysis and the numerical results is somewhat remarkable, given the strong variation of the planetary vorticity in the region. Ultimately, a more formal approach to the problem that includes the meridional vari- 
ation of $f$, the non-zonal nature of the flow and potentially several other features is warranted. In addition, an analysis of the free-slip system would further support or negate the results of the present theory. However, the physics associated with the meridional variation in the deformation radius is quite robust, and I anticipate that shear instability will remain enhanced at the equator. 


\section{Chapter 6}

\section{Conclusion}

\subsection{Summary and Discussion of Thesis}

This thesis examines the dynamics of nonlinear cross-equatorial flow in the context of idealized western boundary currents in the deep ocean. The dynamical problem centers on the transformation of potential vorticity that must occur for fluid parcels to merge smoothly with mid-latitude circulations in the opposite hemisphere. I have found that the system behavior is qualitatively divided between two regimes. The linear, or weakly nonlinear regime is characterized by steady flow, with potential vorticity modification carried out by frictional processes in a laminar boundary layer. This regime has been well-studied by previous theory. At a particular ratio of nonlinearity to dissipation defined by a critical Reynolds number, the system passes a transition to non-steady flow in which eddies form in the western boundary current and are advected southward across the equator where ultimately they dissipate. This thesis identifies the dynamical role of the eddy field as the necessary conduit of vorticity that allows cross-equatorial flow. As the Reynolds number increases beyond the critical value, a turbulent boundary layer emerges that transports vorticity from the inertial portion of the boundary current to the frictional sub-layer that exists adjacent to the boundary where potential vorticity modification is straightforward. In addition, 
this thesis determines that the cause of the eddy field is an instability of the western boundary current that is preferentially triggered at the equator where the internal deformation radius is at its maximum. The mechanism for the instability is suggested to be classical shear instability damped by the presence of viscosity.

Through a scaling of Ertel's potential vorticity from available observations, Chapter 2 determines that an appropriate model for the study of cross-equatorial flow is the shallow-water model. This system has the necessary stretching term in the vorticity equation to distinguish the equator from other latitudes and provides a simple framework for the study of cross-equatorial flow. Dissipation is included as viscous terms in the momentum equations.

To model deep flow in the ocean, the classical model of Stommel and Arons (1960a) is adopted in which fluid is extracted from the model uniformly throughout the domain, which is rectangular in geometry and straddles the equator. To balance the mass sink in the interior of the ocean is a source region located in the north-western corner of the domain. This model necessarily constrains fluid in the western boundary current to cross the equator.

Chapter 3 introduces and describes the details of the particular numerical experiments. The dynamics of the western boundary current are determined by the Reynolds number. For small $R e$, the system is stable and asymptotes to a steady state. The dynamical description of this regime basically follows the original Stommel and Arons (1960a) solution with a Munk layer appended at the western boundary. For Reynolds numbers just above a threshold value, the boundary current becomes unstable and eddies develop just north of the equator. The eddies propagate steadily across the equator in a periodic or quasi-periodic fashion. Only cyclonic eddies emerge at this level of Reynolds number, reflecting the high potential vorticity of the source region. The time-dependent region is confined to approximately one eddy length scale from the western boundary current. As the system becomes still more nonlinear, vorticity anomalies of both signs develop, and the domain of time-dependent activity extends 
quite far into the interior several deformation radii north and south of the equator. There also appears substantial coupling of energy into the equatorial waveguide at this level of forcing.

Despite the intense eddy activity, the time-mean circulation remains qualitatively unchanged from the linear picture. There exists an ever intensifying western boundary current that transports required volume of fluid across the equator as determined by the distribution of the upwelling. Regardless of the Reynolds number, the system is able to respond to carry fluid across and change its potential vorticity.

Vorticity analyses of the time-mean circulation illustrate the dynamical role of the eddy field in modifying potential vorticity and are presented in Chapter 4 . The method examines the flux-conservative form of the time-mean, absolute vorticity (i.e., the mass-weighted potential vorticity) equation in which the vorticity flux vectors are subdivided into three parts, the flux of mean absolute vorticity by the mean velocity field, an eddy flux term, and the flux due to viscosity. Since the vorticity flux vector is non-divergent, it is straightforward to follow vorticity within the domain and observe that a substantial fraction of vorticity advected southward in the western boundary current exits the basin through the western boundary. This process is explained in the context of the linear and weakly nonlinear limits. Examination of the different flux terms in various regions of the domain highlight the essential physics operating in those regions and illustrate the transition from one mechanism of vorticity transfer to another. In the laminar experiments, mean absolute vorticity is advected southward by the western boundary current and is transported westward by the viscous flux. Thus potential vorticity modification is simply a result of the western boundary current being largely a frictional boundary current. At higher Reynolds numbers where inertial effects become important, the viscous boundary layer recedes relative to the full boundary layer and the westward flux is carried through by the eddy term. There appears a turbulent boundary layer which grows with Reynolds number and transports vorticity from the relatively inertial portion of the boundary current to the 
narrow frictional sub-layer.

Numerical experiments carried out at very large Reynolds numbers, $R e=1000$ indicate that the magnitude of the eddy flux term is not bounded by vorticity input into the system. As the system becomes increasingly nonlinear, the eddies must compensate for an increasingly narrow frictional boundary layer and for an eastward flux of vorticity carried by the mean field. This behavior contrasts with the convective problem in which the eddy flux is bounded by the buoyancy forcing applied to the system.

One feature that emerges in the numerical experiments at high Reynolds number is a recirculation in the vicinity of the equator. This recirculation is not associated with trapped fluid of constant potential vorticity as are highly nonlinear recirculations in mid-latitude models. Instead it appears to be a region of enhanced eddy formation or perhaps a region of retarded eddy propagation. This eddy-driven feature has the dynamical role of contributing to the necessary westward eddy flux of vorticity in this important region just north of the equator. Thus both the mid-latitude and equatorial recirculations are essential dynamical elements in the vorticity balance, but their character is quite different.

The role of the eddy field is quite insensitive to details of the model. Numerical experiments with different upwelling distributions and different boundary conditions present a fundamentally similar impression. Regardless of the volume of fluid that is required to penetrate into the opposite hemisphere, the eddies are able to transport the necessary potential vorticity to permit the flow. The boundary conditions merely establish approximately the latitude where the zonally integrated vorticity flux must vanish. For a no-slip condition, it is the equator, and for free-slip, it is south of the equator, but not indefinitely south.

Finally, Chapter 5 investigates the cause of the eddy field itself. Since the dynamics of the cross-equatorial flow rest fundamentally on the existence of an eddy field to transport vorticity westward to the boundary, it is desirable to understand the mech- 
anism that creates them. A linear stability analysis of an idealized western boundary current indicates that the flow is unstable to inviscid shear instability. The boundary velocity has an inflection point in its profile and therefore does not satisfy the sufficient conditions for stability. Adding viscosity to the analysis damps the growth of the instability, but does not qualitatively alter its features. The meridional wavenumber associated with the maximum growth rate changes slightly, but the eigenfunctions of the instability are nearly identical.

An interesting feature that emerges from the numerical model as well as from observations and the analysis is that the instability of the western boundary current is tropically enhanced. The maximum in the growth rate occurs in the vicinity of the equator. A slight asymmetry between hemispheres results from the fact that the absolute vorticity of the western boundary current is not symmetric about the equator. The physical explanation for this equatorial enhancement centers on the increased scale of the equatorial deformation radius. The meridional variation in the deformation radius shifts the phase speeds of waves that propagate on the vorticity gradient of the mean flow such that the adjacent disturbances at the equator have the largest wavenumber and can mutually reinforce one another most effectively.

It is important to reiterate the motivation for this work. Observations indicate that fluid travels between hemispheres, necessarily across the equator in deep western boundary currents. Although observations are insufficient to determine the mechanism for vorticity transformation, it is certain that at some point along the current path, the vorticity is modified. If the deep western boundary currents are frictionally dominated, then the classical studies of the abyssal circulation are sufficient to explain the dynamics. However, scaling arguments from observations indicate that the boundary currents are at least partially inertial, in which case the present study applies directly.

The model predicts the formation of time-dependent features at a critical Reynolds number. Variability is observed in many measurements of the tropical oceans, at all 
depths. A dominant time-scale for the variability is from 10 to 70 days. The model also finds variability on these time-scales. It is the scale of the eddy features that naturally emerge in the model. However, these model features can be tuned by parameter settings, and without clear understanding of the lateral viscosity of the ocean, it is difficult to make direct comparisons other than to say that the sets of features are analogous and probably generated identically.

Based on the above calculations of critical Reynolds numbers, it is possible to estimate the critical lateral viscosity at which the western boundary goes unstable. Using the definition $R e=\frac{S_{0}}{H A_{H}}$ let $R e_{c}=30$. Then for a deep western boundary current that is approximately $1000 \mathrm{~m}$ deep and that transports $20 \mathrm{~Sv}$ across the equator, the critical viscosity at which variabity would set in would be $A_{H}=670 \mathrm{~m}^{2} \mathrm{~s}^{-1}$.

\subsection{Extension to Full Three-Dimensional Fluid}

It is tempting to explore the process of cross-equatorial flow using a more realistic, fully three-dimensional ocean model. Of course, such an experiment would retain substantial dynamical constraints that are omitted using the shallow-water model. However, I believe that the basic description described above would remain essentially unaltered by such a study.

Fundamentally, the vorticity flux constraint remains identical whether describing a two- or three-dimensional fluid. The formalism of the $\mathbf{J}$-vector analysis clarifies this point. The impermeability theorem states that mass-weighted potential vorticity, $\rho q$, can not be transported across isentropic surfaces (i.e., surfaces of constant potential density) (Haynes and McIntyre (1987)). Thus the three-dimensional fluid is conveniently decomposed into a series of layers, each one analogous to the shallow-water system described in this thesis.

The form of the vorticity flux vector takes a slightly different form for a threedimensional fluid. In the notation of Marshall and Nursur (1992), the potential vor- 
ticity equation can be written

$$
\frac{\partial}{\partial t}(\rho q)+\nabla \cdot \mathbf{J}=0
$$

where $\rho$ is the density,

$$
q=-\frac{1}{\rho} \omega \cdot \nabla \sigma
$$

the potential vorticity, $\omega$ the full abolute vorticity, and $\sigma$ the potential density anomaly. The $\mathbf{J}$-vector in this system is defined by

$$
\mathbf{J}=\rho q \mathbf{u}+F \times \nabla \sigma-\frac{1}{g} B \omega
$$

in which $F$ encompasses all nonconservative (i.e., viscous) body forces, and $B$ is the buoyancy forcing term,

$$
B=-\frac{1}{g} \frac{D \sigma}{D t} .
$$

In a three-dimensional model, the $\mathbf{J}$-vector comprises an advective component, a frictional term, and a contribution due to non-adiabatic density changes.

It is possible to demarcate sub-regions within each layer and require again that fluid flowing across the equator between isentropic sheets transform its potential vorticity just as in the shallow-water model. As a result there arises an identical requirement to flux vorticity westward between different portions of the boundary current.

Since it is the last term in Equation 6.3 that distinguishes a three-dimensional from a two-dimensional model in terms of the vorticity flux, I concentrate on its effect only. In the absence of observational evidence that the tropics are strongly non-adiabatic, it is worth noting that in the adiabatic limit, this term vanishes, and the system remains identical to the shallow-water system. If a buoyancy flux exists, its ability to flux vorticity westward is proportional to the buoyancy itself and the westward component of absolute vorticity. Even if the buoyancy is substantial the horizontal component of vorticity is quite small in the absence of a large vertical shear. Thus it is unlikely that this term contributes significantly to the westward vorticity flux in a full three-dimensional fluid. 
This line of reasoning is supported by the numerical experiments of Haine and Marshall (1996) in which the both symmetric and baroclinic instability act on a differentially surface-cooled fluid in an $f$-plane channel. In that work, symmetric instability which has a very fast time scale is initially the primary mechanism for vorticity transfer. However, baroclinic instability ultimately dominates the lateral transfer of vorticity. This process is fundamentally more efficient than symmetric instability and effectively shuts down the latter process. Symmetric instability is closely related to the inertial instability that may be present in the tropical region in a full threedimensional fluid, and baroclinic instability is quite similar to barotropic instability. Thus it seems reasonable to expect that although the dynamics may be slightly altered by a local buoyancy forcing in a three-dimensional fluid, its contribution to the vorticity flux will be small compared to the eddies that emerge as a result of the shear instability of the western boundary current. Furthermore, numerical experiments using a three-dimensional primitive equation model by Kawase (1992) show basically the same behavior observed in the shallow-water system. The boundary current becomes unstable at a particular strength of the forcing, eddies develop, and propagate across the equator. There is no indication that the buoyancy forcing term is significant. 


\section{Appendix A}

\section{Numerical Approximations}

The time-dependent numerical model is the central tool by which I analyze crossequatorial flow. Although the spatial discretization and issue of boundary conditions are described in Chapter 2, several important features of the numerics are not included in that discussion. This appendix completes the description of the model, clarifying the time-stepping scheme used, the method of stretching the model grid, and the numerical approximatins of the vorticity and $\mathbf{J}$-vector components that are essential to the analysis.

\section{A.1 Model Time-Stepping}

The above discretization uses center-differencing, and it maintains second order spatial accuracy. For time stepping, the essential feature is stability and efficiency over accuracy. To satisfy criteria such as the CFL condition and a similar constraint due to friction, the time step must be quite short relative to the time scale of the motion. The more severe limitation for numerical solutions of geophysical systems in general is the spatial accuracy.

Arguably, the simplest explicit time-stepping scheme that is stable is the threelevel modified Adams-Bashforth (AB2) algorithm. Each future state is determined 
by specific knowledge of the preceding two states according to an approximation to an integral equation. A readable discussion of the original scheme and comparison to other time stepping algorithms is found in Mesinger and Arakawa (1976).

The most general time-stepping problem is written in the continuous form

$$
\psi_{t}=\Phi(\psi(t))
$$

in which the function $\Phi$ comprises all of the terms that contribute to the time rate of change of a variable, $\psi$. Now letting superscripts denote time steps, $t=n \Delta t$, the discretization should approximate

$$
\psi^{n+1}-\psi^{n}=\int_{t}^{t+1} \Phi d t^{\prime} .
$$

The original AB2 scheme approximates the right hand side of Eq. A.2 as the value of $\Phi$ at its midpoint multiplied by $\Delta t$, as in the leapfrog scheme, but uses a Taylor expansion about the time $n \Delta t$ to extrapolate forward in time and determine its value:

$$
\begin{aligned}
\frac{1}{\Delta t} \int_{n \Delta t}^{(n+1) \Delta t} \Phi d t^{\prime} & \cong \Phi^{n+\frac{1}{2}} \\
& \cong \Phi^{n}+\frac{1}{2} \Delta \Phi^{n} \\
& \cong \frac{3}{2} \Phi^{n}-\frac{1}{2} \Phi^{n-1}
\end{aligned}
$$

Standard analysis of this scheme using a simple oscillation equation reveals that two modes exist, one real and one computational, as expected from the fact that this routine is three-level. The unphysical mode is damped, but the real mode is unstable, for all time-steps. This feature is not unlike the instability associated with two-level forward differencing (Euler) in which the term on the right hand side of Eq A.2 is simply approximated by $\Phi^{n}$. Just as that instability is relieved by using the implicit formulation,

$$
\frac{1}{\Delta t} \int_{n \Delta t}^{(n+1) \Delta t} \Phi d t^{\prime} \cong \Phi^{n+1},
$$

the instability of the AB2 scheme is also relieved by shifting forward the time at which the forcing is to be determined. Thus the modified AB2 scheme extrapolates to a time 
slightly ahead of the mid-point between time steps. Specifically, the time-stepping takes the form

$$
\frac{\psi^{t+1}-\psi^{t}}{\Delta t}=\left(\frac{3}{2}+\epsilon\right) \Phi^{t}-\left(\frac{1}{2}+\epsilon\right) \Phi^{t-1},
$$

where $\epsilon$ is a small number ( 0.1 in this study).

\section{A.2 Grid Stretching}

The original model was specified on a uniform grid directed toward general atmospheric problems. Present knowledge of oceanographic motions in confined basins allows an improvement in the efficiency of the code by stretching the grid and concentrating resolution in regions in which the motion is characterized on small spatial scales.

Stretching a grid can be as simple as varying grid dimensions and using the same discretization. However, as discussed in Marti et al. (1992), changing only the grid lengths loses the second-order accuracy of the center-differencing. Grid elements no longer lie at midpoints, where averaging and differential operations take place.

Finite difference formulations follow from Taylor expansions. For the general function, $f(x)$, and letting primes denote derivatives

$$
\begin{aligned}
f\left(x+\Delta x_{1}\right)= & f(x)+\Delta x_{1} f^{\prime}(x)+ \\
& \frac{\left(\Delta x_{1}\right)^{2}}{2 !} f^{\prime \prime}(x)+\frac{\left(\Delta x_{1}\right)^{3}}{3 !} f^{\prime \prime \prime}(x) \cdots \\
f\left(x-\Delta x_{2}\right)= & f(x)-\Delta x_{2} f^{\prime}(x)+ \\
& \frac{\left(\Delta x_{2}\right)^{2}}{2 !} f^{\prime \prime}(x)-\frac{\left(\Delta x_{2}\right)^{3}}{3 !} f^{\prime \prime \prime}(x) \cdots
\end{aligned}
$$

The averaging operator is formed by adding Eqs. A.6a-A.6b, and the differential operator by subtracting them. Rearranging terms,

$$
\begin{aligned}
f(x)= & \frac{1}{2}\left(f\left(x+\Delta x_{1}\right)+f\left(x-\Delta x_{2}\right)+\right. \\
& \left.\left(\Delta x_{1}-\Delta x_{2}\right) f^{\prime}(x)+O\left(\Delta x_{1}, \Delta x_{2}\right)_{\max }\right)
\end{aligned}
$$




$$
\begin{aligned}
f^{\prime}(x)= & \frac{1}{\Delta x_{1}+\Delta x_{2}}\left(f\left(x+\Delta x_{1}\right)-\right. \\
& f\left(x-\Delta x_{2}\right)+\left(\frac{\left(\Delta x_{1}\right)^{2}}{2 !}-\frac{\left(\Delta x_{2}\right)^{2}}{2 !}\right) f^{\prime \prime}(x)+ \\
& \left.O\left(\left(\Delta x_{1}\right)^{3},\left(\Delta x_{2}\right)^{3}\right)_{\text {max }}\right) .
\end{aligned}
$$

Clearly, when increments of $x$ are equal, the schemes are of second order accuracy, but not when $\Delta x_{i}$ are unequal. The only grid for which all elements lie at midpoints of adjacent ones is the uniform grid. To maintain the accuracy of the discretization on a stretched grid requires an analytical mapping of the grid. Following Marti et al. (1992), the location of each grid point is determined by an analytic function (e.g., $x=x(i))$ such that, according to the chain rule,

$$
\frac{d}{d i}=\frac{d x}{d i} \frac{d}{d x} .
$$

To examine the accuracy of a scheme using this mapping, Taylor expand the function, $g=g(i)$ about the integral index $i$ :

$$
\begin{aligned}
& g(i+1)=g(i)+g^{\prime}(i)+\frac{1}{2} g^{\prime \prime}(i)+\frac{1}{6} g^{\prime \prime \prime}(i)+\cdots \\
& g(i-1)=g(i)-g^{\prime}(i)+\frac{1}{2} g^{\prime \prime}(i)-\frac{1}{6} g^{\prime \prime \prime}(i)+\cdots
\end{aligned}
$$

As before,

$$
\begin{aligned}
g(i) & =\frac{1}{2}(g(i+1)+g(i-1))+O\left(g^{\prime \prime}\right) \\
g^{\prime}(i) & =(g(i+1)-g(i-1))+O\left(g^{\prime \prime \prime}\right)
\end{aligned}
$$

So the mapped scheme is second order accurate in $g(i)$. The chain rule determines that

$$
\begin{aligned}
& \frac{d^{2} g}{d i^{2}}=\left(\frac{d x}{d i}\right)^{2} \frac{d^{2} g}{d x^{2}}+\frac{d^{2} x}{d i^{2}} \frac{d g}{d x} \\
& \frac{d^{3} g}{d i^{3}}=\left(\frac{d x}{d i}\right)^{3} \frac{d^{3} g}{d x^{3}}+3 \frac{d^{2} x}{d i^{2}} \frac{d x}{d i} \frac{d^{2} g}{d x^{2}}+\frac{d^{3} x}{d i^{3}} \frac{d g}{d x} .
\end{aligned}
$$


And it is clear that the scheme is second order accurate in $g(x)$ as long as

$$
\begin{gathered}
O\left(\frac{d^{2} x}{d i^{2}}\right) \leq O\left(\left(\frac{d x}{d i}\right)^{2}\right) \\
O\left(\frac{d^{3} x}{d i^{3}}\right) \leq O\left(\left(\frac{d x}{d i}\right)^{3}\right) \\
O\left(\frac{d^{2} x}{d i^{2}} \frac{d x}{d i}\right) \leq O\left(\left(\frac{d x}{d i}\right)^{3}\right) .
\end{gathered}
$$

As long as the change in the variation of the grid is sufficiently small, the scheme remains second order accurate. The argument extends straightforwardly to more than one dimension.

In the present model, I chose, using $i$ and $j$ as the zonal and meridional indices,

$$
x=\alpha_{x} \tanh \left(s_{x}\left(i-i_{0}\right)\right)+\gamma_{x}
$$

and

$$
y=\alpha_{y}\left(\tanh \left(s_{y}\left(j-j_{0}\right)\right)+\tanh \left(s_{y}\left(j-j_{1}\right)\right)\right)+\gamma_{y}
$$

The constants $\alpha_{i}$ and $\gamma_{i}$ provide shift and scaling so as to match the two desired boundaries, $s_{i}$ represent the degree of stretching, and $i_{0}$ and $j_{0}$ represent shifts for the hyperbolic tangent function to allow a reduction of the grid separation near the eastern and northern boundaries as well as near the west and equator.

For the runs presented below, using $N$ and $M$ to denote number of zonal and meridional grid points, $s_{x}=1.85 / N, s_{y}=4.3 / M, i 0=4 N / 5, j 0=2 M / 21$. The parameter, $j_{1}=M-j_{0}$, for symmetry about the equator. Figures A.1 and A.2 present the grids used in the standard runs of these experiments, with $N=255$ and $M=511$, along with their derivatives. Relations A.12a - A.12c are satisfied by these functions. Numerical model runs in Chapter 5 only use $N=127$ and $M=255$, which are deemed adequate to demonstrate the development of instability in the vicinity of the equator. 

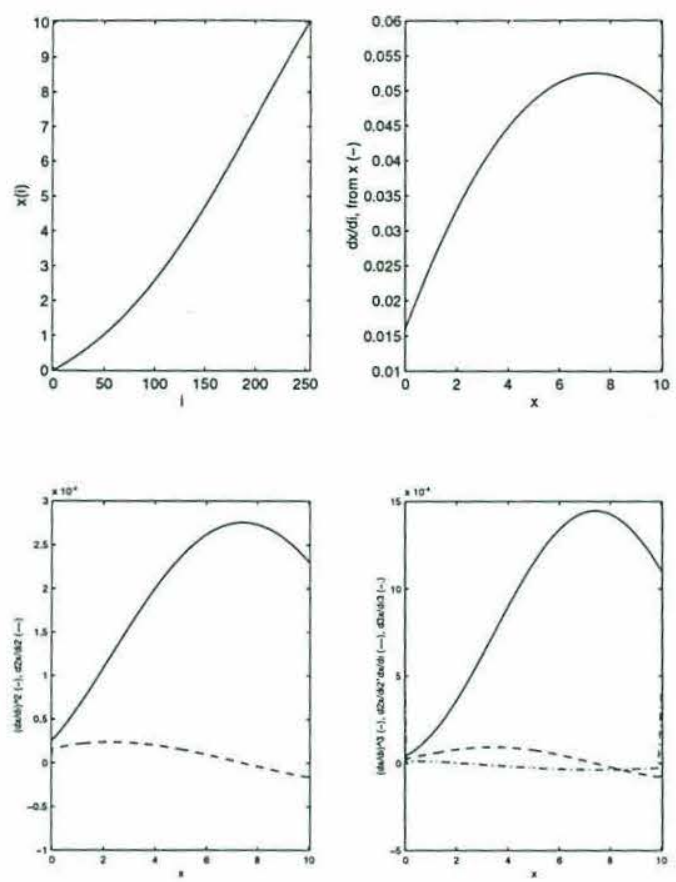

Figure A.1: Grid used in the $x$-direction with its derivatives using $s_{x}=1.5 / N$, with $N=256$.
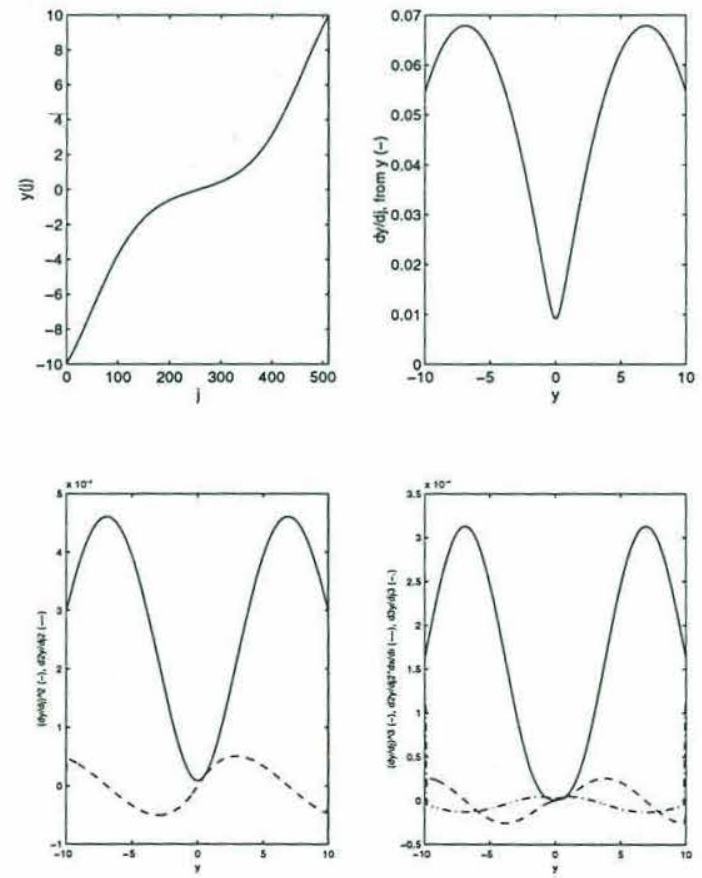

Figure A.2: Grid used in the $y$-direction with its derivatives using $s_{y}=5 / M$, with $M=511$. 


\section{A.3 Numerical Approximation to Vorticity Com- ponents}

Although the decomposition of the continuous system is straightforward, and the vorticity and flux balances consist only of a few terms, some errors result from the discretization. The numerical algorithm is designed to conserve potential enstrophy, not to step forward the exact vorticity equation. As these errors are a result of the discretization, their magnitude is grid-scale dependent. In addition, they have some spatial structure as shown in a similar system by Boudra and Chassignet (1988).

The numerical approximation to the vorticity equation is

$$
\frac{\partial \zeta}{\partial t}+\delta_{x}\left(\bar{q}^{x} \bar{U}^{x y}\right)+\delta_{y}\left(\bar{q}^{y} \bar{V}^{x y}\right)=A_{H}\left(\delta_{x} \delta_{x} \zeta+\delta_{y} \delta_{y} \zeta\right)
$$

Errors are incurred because, for example,

$$
\delta_{x}\left(\bar{q}^{x} \bar{U}^{x y}\right) \neq \bar{u}^{y} \delta_{x}{\overline{\zeta_{A}}}^{x}+\zeta_{A} \delta_{x} \bar{u}^{x y}
$$

Some algebra reveals, noting the commutability of the averaging and difference operators, that

$$
\begin{aligned}
\delta_{x}\left(\bar{q}^{x} \bar{U}^{x y}\right)= & \bar{q}^{x x}\left\{\bar{u}^{x x y} \delta_{x} \bar{h}^{x x y}+\bar{h}^{x x x y} \delta_{x} \bar{u}^{x y}+\right. \\
& \left.\frac{1}{4} \delta_{x}\left[\delta_{y} \bar{u}^{x} \delta_{y} \bar{h}^{x x}+\overline{\delta_{x} u \delta_{x} \bar{h}^{x y}}\right]\right\}+ \\
& \left.\bar{U} x \underline{\frac{1}{\bar{h}^{x x y}} \delta_{x}\left(\bar{y}^{x}+\bar{\zeta}\right.}\right)+\left(\bar{\zeta}^{x x}+\bar{\zeta}^{x x}\right) \delta_{x} \frac{1}{\bar{h}^{x x y}}+ \\
& \left.\frac{1}{4} \delta_{x}\left[\delta_{x} \zeta_{A} \delta_{x} \frac{1}{\bar{h}^{x y}}\right]\right\}
\end{aligned}
$$

Some terms in Eq. A.17 are recognizable analogs to those of the continuous vorticity equation. However, some terms relating to the horizontal gradients of layer thickness have no such analog. They are simply a result of the choice of potential enstrophy conservation in the discretization. As a result, in the analysis of the vorticity budget and $\mathbf{J}$-vectors, these errors must be considered. 
Following the method of Boudra and Chassignet (1988), I decompose the vorticity equation into five components, corresponding to relative vorticity advection, planetary vorticity advection, stretching, friction, and the combined extra terms due to the conservation properties of the scheme. Specifically,

$$
\begin{aligned}
& R V A=\bar{U}^{x x y}{\overline{\frac{1}{\bar{h}^{x x y}}}}^{x} \delta_{x} \bar{\zeta}^{x}+{\overline{V^{x y y}}}^{\frac{1}{\bar{h}^{x y y}} \delta_{y}} \bar{\zeta}^{y} \\
& P V A=\bar{U}^{x x y} \overline{\frac{1}{\bar{h}^{x x y}}} \delta_{x} \bar{f}^{x}+\bar{V}^{x y y} \overline{\frac{1}{\bar{h}^{x y y}}} \delta_{y} \bar{f}^{y} \\
& S T R=\bar{q}^{x x} \bar{h}^{x x x y} \delta_{x} \bar{u}^{x y}+\bar{q}^{y y} \bar{h}^{x y y y} \delta_{y} \bar{v}^{x y} \\
& F R I C=-A_{H}\left[\delta_{x} \delta_{x} \zeta+\delta_{y} \delta_{y} \zeta\right] \\
& \text { EXTRA }=\bar{q}^{x x}\left\{\bar{u}^{x x y} \delta_{x} \bar{h}^{x x y}+\frac{1}{4} \delta_{x}\left[\delta_{y} \bar{u}^{x} \delta_{y} \bar{h}^{x x}+\overline{\delta_{x} u \delta_{x} \bar{h}^{x y}}\right]\right\}+ \\
& \bar{U}^{x x y}\left\{{\overline{\zeta_{A}}}^{x x} \delta_{x} \frac{1}{\bar{h}^{x x y}}+\frac{1}{4} \delta_{x}\left[\delta_{x} \zeta_{A} \delta_{x} \frac{1}{\bar{h}^{x y}}\right]\right\}+ \\
& \bar{q}^{y y}\left\{\bar{v}^{x y y} \delta_{y} \bar{h}^{x y y}+\frac{1}{4} \delta_{y}\left[\delta_{x} \bar{v}^{y} \delta_{x} \bar{h}^{y y}+\overline{\delta_{y} v \delta_{y} \bar{h}^{y^{x}}}\right]\right\}+ \\
& \bar{V}^{x y y}\left\{{\overline{\zeta_{A}}}^{y y} \delta_{y} \frac{1}{\bar{h}^{x y y}}+\frac{1}{4} \delta_{y}\left[\delta_{y} \zeta_{A} \delta_{y} \frac{1}{\bar{h}^{x y}}\right]\right\} .
\end{aligned}
$$

These are the forms of relative vorticity advection, planetary vorticity advecting, stretching, and vorticity associated with friction that the model conserves. The EXTRA term is simply the result of the discretization of the model and has no physical counterpart. In the experiments RVA, PVA, STR, and FRIC are determined, and their residual calculated. For all runs in this study, the resolution of the model is sufficiently high that the residual is negligible compared to the components that contribute to it. All vorticity balance components are determined at $\zeta$-points of the C-grid model.

\section{A.4 Numerical Approximation to J-vectors}

As with the components of vorticity, the $\mathbf{J}$-vectors themselves can also be evaluated in terms of the discretization. The model ensures that the numerical divergence of the time averaged vector field

$$
\mathbf{J}=\left(\bar{q}^{x} \bar{U}^{x y}-A_{H} \delta_{x} \zeta, \bar{q}^{y} \bar{V}^{x y}-A_{H} \delta_{y} \zeta\right)
$$


vanishes. As a result, I define

$$
\begin{aligned}
\mathbf{J}_{m n} & =\left(\overline{\bar{q}^{x t} \bar{U}^{x y t} y}, \overline{\bar{q}^{y t} \bar{V}^{x y t}}\right) \\
\mathbf{J}_{e d} & =\left(\overline{\bar{q}^{x} \bar{U}^{x y} y t}, \overline{\bar{q}^{y} \bar{V}^{x y}}\right)-\mathbf{J}_{m n} \\
\mathbf{J}_{f r i c} & =-A_{H}\left(\delta_{x} \bar{\zeta}^{y t}, \delta_{y} \bar{\zeta}^{x t}\right) .
\end{aligned}
$$

The J-vector components lie at $h$-points of the model. 


\section{References}

Adcroft, A. J. 1995. Numerical algorithms for use in a dynamical model of the ocean. Ph.D. thesis, Imperial College, University of London.

Arakawa, A., and Lamb, V. R. 1978. Finite difference scheme for the nonlinear shallow-water equations. Unpublished Manuscript.

Bainbridge, A. E. 1980. Geosecs atlantic expedition. National-Science Foundation, Vol 2.

Bender, C. M., and Orszag, S. A. 1978. Advanced mathematical methods for scientists and engineers. McGraw-Hill. 593 pp.

Boudra, D. B., and Chassignet, E. P. 1988. Dynamics of Agulhas retroflection and ring formation in a numerical model. part i: The vorticity balance. J. Phys. Ocean., 18, 280-303.

Bryan, K. A. 1963. A numerical investigation of a nonlinear model of a wind-driven ocean. J. Atm. Sci., 20, 594-606.

Cessi, P., and Young, G. R. 1993. Nonlinear disturbances of western boundary currents. J. Phys. Ocean., 23, 1727-1735.

Drazin, P. G., and Reid, W. H. 1981. Hydrodynamic stability. Cambridge University Press. 527 pp. 
Haine, T. W. N., and Marshall, J. C. 1996. Gravitational, symmetric and baroclinic instability of the ocean mixed layer. Submitted to J. Phys. Ocean.

Haltiner, G. J., and Williams, R. T. 1980. Numerical prediction and dynamic meterology. 2nd edn. John Wiley and Sons. 477 pp.

Haynes, P. H., and McIntyre, M. E. 1987. On the evolution of vorticity and potential vorticity in the presence of diabatic heating or other forces. J. Atm. Sci., 44, $828-841$.

Ierley, G. R. 1987. On the onset of inertial recirculation in barotropic general circulation models. J. Phys. Ocean., 17, 2366-2374.

Ierley, G. R. 1990. Boundary layers in the general ocean circulation. Ann. Rev. Fluid Mech., 22, 111-142.

Ierley, G. R., and Young, W. R. 1991. Viscous instabilities in the western boundary layer. J. Phys. Ocean., 21, 1323-1332.

Johns, W. E., Fratantoni, D. M., and Zantopp, R. J. 1993. Deep western boundary current variability off northeastern brazil. Deep-Sea Res., 40, 293-310.

Kamenkovich, V. M., Sheremet, V. A., Pastushkov, A. R., and Belotserkovsky, S. O. 1995. Analysis of the barotropic model of the subtropical gyre in the ocean for finite Reynolds numbers. part i. J. Mar. Res, 53, 959-994.

Kawase, M. 1987. Establishment of deep ocean circulation driven by deep-water production. J. Phys. Ocean., 17, 2294-2317.

Kawase, M., and Sarmiento, J. L. 1986. Circulation and nutrients in middepth Atlantic waters. J. Geophys. Res, 91, 9749-9770.

Kawase, M., Rothstein, L. M., and Springer, S. R. 1992. Encounter of the deep western boundary current with the equator: A numerical spin-up experiment. J. Geophys. Res, 97, 5447-5463. 
Kundu, P. K. 1990. Fluid mechanics. Academic Press. 638 pp.

Kunze, E., and Sanford, T. B. 1996. Abyssal mixing: Where it is not. J. Phys. Ocean., 26, 2286-2296.

Lipps, F. B. 1963. Stability of jets in a divergent barotropic fluid. J. Atm. Sci., 20, $120-129$.

Marshall, J. C. 1981. On the treatment of the lateral boundaries in quasi-geostrophic ocean models. Unpublished manuscript.

Marshall, J. C., and Nurser, A. J. G. 1992. Fluid dynamics of oceanic thermocline ventilation. J. Phys. Ocean., 22, 583-595.

Marti, O., Madec, G., and Delecluse, P. 1992. Comment on "Net Diffusivity in Ocean General Circulation Models with Nonuniform Grids" by F. L. Yin and I. Y. Fung. J. Geophys. Res, 97, 12763-12766.

McCartney, M. S. 1993. Crossing of the equator by the deep western boundary current in the western Atlantic ocean. J. Phys. Ocean., 23, 1953-1974.

Mesinger, F., and Arakawa, A. 1976. Numerical methods used in atmospheric models. Global Atmospheric Research Programme (GARP). GARP Publications Series, No. 17, Volume I.

Munk, W. H. 1950. On the wind-driven ocean circulation. J. Meteor., 7, 79-93.

$\mathrm{Ni}, \mathrm{B}$. 1996. Temporal, spatial and pulse instabilities of the gulf stream. Ph.D. thesis, Massachusetts Institute of Technology and Woods Hole Oceanographic Institute Joint Program in Oceanography.

Pedlosky, J. 1987a. Geophysical fluid dynamics. 2nd edn. Springer-Verlag. 710 pp.

Pedlosky, J. 1987b. An inertial theory of the equatorial undercurrent. J. Phys. Ocean., $17,1978-1985$. 
Philander, S. G. 1990. El niño, la niña, and the southern oscillation. Academic Press. 289 pp.

Philander, S. G. H. 1976. Instabilities of zonal equatorial currents. J. Geophys. Res, 81, 3725-3735.

Rayleigh, Lord. 1880. On the stability, or instability, of certain fluid motions. Proc. London Math. Soc., 11, 57-70.

Richardson, P. L., and Schmitz, Jr., W. J. 1993. Deep cross-equatorial flow in the Atlantic measured with SOFAR floats. J. Geophys. Res, 98, 8371-8387.

Ripa, P. 1983. General stability conditions for zonal flows in a one-layer model on the $\beta$-plane or the sphere. J. Fluid Mech., 126, 463-489.

Sadourny, R. 1975. Compressible model flows on the sphere. J. Atm. Sci., 32, 21032110.

Sheremet, V. A., Kamenkovich, V. M., and Pastushkov, A. R. 1995. Analysis of the barotropic model of the subtropical gyre in the ocean for finite Reynolds numbers. part ii. J. Mar. Res, 53, 995-1024.

Springer, S. R., and Kawase, M. 1993. Nonlinear and dissipative dynamics in the connection region between western boundary currents and equatorial currents. $J$. Geophys. Res, 98, 12511-12525.

Stern, M. 1961. The stability of thermoclinic jets. Tellus, 13, 503-508.

Stommel, H, and Arons, A. B. 1960a. On the abyssal circulation of the world ocean-i. stationary planetary patterns on a sphere. Deep-Sea Res., 6, 140-154.

Stommel, H, and Arons, A. B. 1960b. On the abyssal circulation of the world oceanii. an idealized model of the circulation pattern and amplitude in oceanic basins. Deep-Sea Res., 6, 217-233. 
Weiss, R. F., Bullister, J. L., Gammon, R. H., and Warner, M. J. 1985. Atmospheric chlorofluoromethanes in the deep equatorial Atlantic. Nature, 314, 608-610.

Weiss, R. F., Warner, M. J., and Harrison, K. G. 1989. Deep equatorial atlantic chlorofluorocarbon distributions. Eos, Trans. Am. Geophys. Union, 70, 1132.

Weiss, R. F., Bullister, J. L., Van Woy, F. A., Warner, M. J., Salameh, P. K., and Gammon, R. H. 1991. Transient tracers in the ocean, tropical atlantic study: Chlorofluorocarbon measurements. Tech. rept. Scripps Institution of Oceanography. Reference Series, 91-1.

Weiss, R. F., Warner, M. J., Salameh, P. K., Van Woy, F. A., and Harrison, K. G. 1993. South atlantic ventilation experiment: Sio chlorofluorocarbon measurements. Tech. rept. Scripps Institution of Oceanography. Reference Series, 93-49. 